\title{
Formulação do Método dos Elementos de Contorno e Plasticidade com Gradiente
}

\author{
Carlos Alberto Fudoli \\ Tese apresentada à Escola de Engenharia de São Carlos, da Universidade de São Paulo, \\ como parte dos requisitos para obtenção do título de \\ Doutor em Engenharia de Estruturas
}

Orientador: Prof. Tit. Wilson Sergio Venturini

São Carlos

1999 
Class.

Cutt.

2286

Tombo

$166 / 99$

Ficha catalográfica preparada pela Seção de Tratamento da Informação do Serviço de Biblioteca - EESC/USP

F952f

Fudoli, Carlos Alberto

Formulação do método dos elementos de contorno e plasticidade com gradiente / Carlos Alberto Fudoli. -- São Carlos, 1999.

Tese (Doutorado) -- Escola de Engenharia de São Carlos-Universidade de São Paulo, 1999.

Área: Engenharia de Estruturas.

Orientador: Prof. Dr. Wilson Sergio Venturini.

1. Método dos elementos de contorno. 2. Problemas não lineares. 3. Localização de deformações.

I. Título. 
Candidato: Engenheiro CARLOS ALBERTO FUDOLI

Tese defendida e aprovada em 20-08-1999

pela Comissão Julgadora:

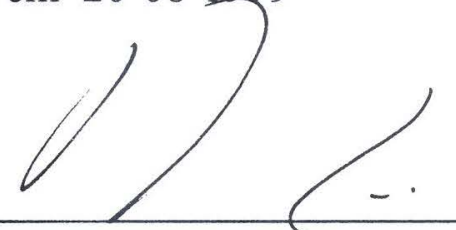

Prof. Titular WILSON SERGIO VENTURINI (Orientador)

(Escola de Engenharia de São Carlos - Universidade de São Paulo)

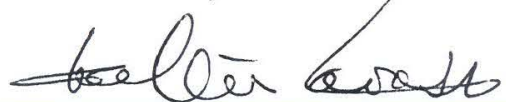

Prof. Titular WALTER SAVASSI

(Escola de Engenharia de São Carlos - Universidade de São Paulo)

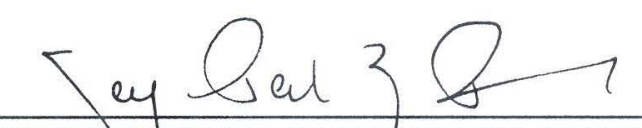

Prof. Associado SERGIO PERSIVAL BARONCINI PROENÇA

(Escola de Engenharia de São Carlos - Universidade de São Paulo)

$$
\{\sigma\rangle 1 \underbrace{2} \downarrow \text {. }
$$

Prof. Livre-Docente EUCLIDES DE MESQUITA NETO

(UNICAMP - Universidade Estadual de Campinas)

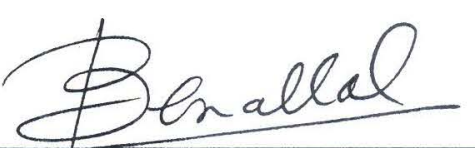

Prof. Doutor AHMED BENALLAL

(Université Paris)

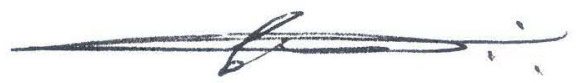

Prof. Titular CARLITO CALIL JUNIOR

Coordenador da Área de Engenharia de Estruturas

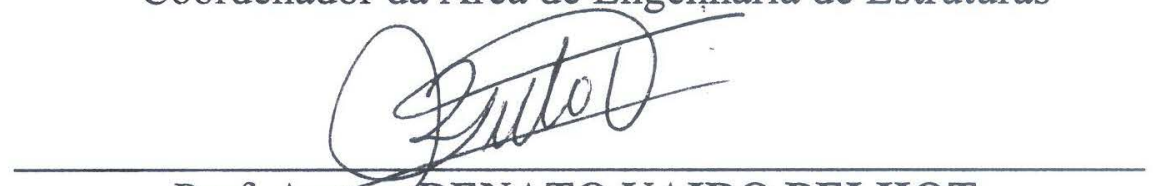

Prof. Assoc. RENATO VAIRO BELHOT

Vice-Presidente da Comissão de Pós-Graduação da EESC em exercício 
À Tania e

Alessandra 


\section{AGRADECIMENTOS}

Aos colegas, professores e funcionários do Departamento de Estrutura da EESC-USP. Em especial, aos meus dois grandes mestres, os professores Wilson Sergio Venturini e Sergio P. B. Proença, com seus estilos diferentes, e com os quais tive o prazer de conviver e aprender durante esse tempo, e cuja seriedade e dedicação procuro imitar. Agradeço a oportunidade que me proporcionaram de praticar um pouco a aventura da pesquisa científica ou tecnológica. Em especial ao prof. Venturini, por sua orientação realmente participativa.

Ao Laboratoire de Mécanique et Technologie (ENS-Cachan/CNRS/Paris 6), sobretudo nas figuras dos professores Ahmed Benallal, que colaborou na elaboração do trabalho, e René Billardon.

Ao CNPQ, que possibilitou aquela passagem pelo LMT, quando tive a oportunidade de aprender outras tantas coisas. E à CAPES, pela bolsa no Brasil.

Os recursos disponíveis no CENAPAD-SP/UNICAMP foram essenciais na parte computacional do trabalho. Mas agradeço sobretudo aos colegas, com os quais tenho tido o privilégio de trabalhar aprendendo, especialmente à Ana C. C. Drummond.

Minha gratidão sobretudo à Tania, cujo apoio, compreensão e incentivo foram fundamentais durante toda a realização deste trabalho. Meus agradecimentos aos nossos pais, pelas mesmas razões. 


\section{SUMÁRIO}

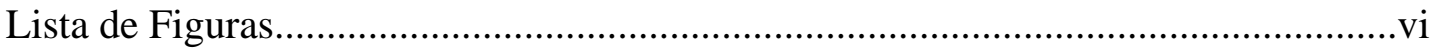

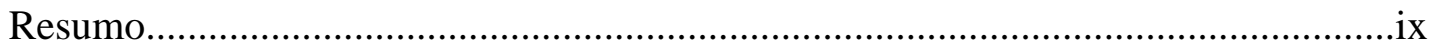

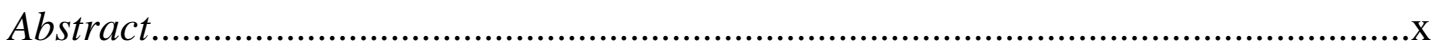

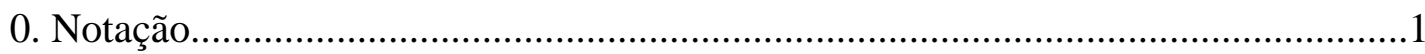

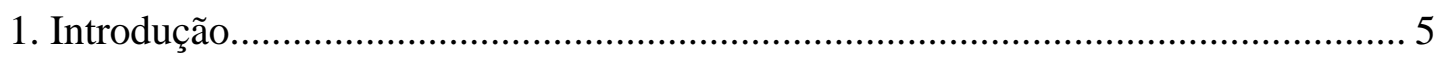

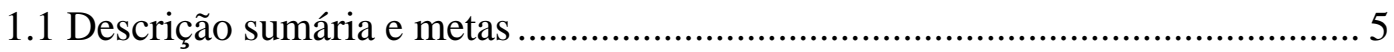

1.1.1 Localização de deformações ................................................................. 5

1.1.2 Método dos elementos de contorno........................................................ 7

1.1.3 Objetivos e delimitação........................................................................ 8

1.1.4 Descrição do texto.................................................................................. 10

$1.2 \mathrm{O}$ problema da localização de deformações ..................................................... 11

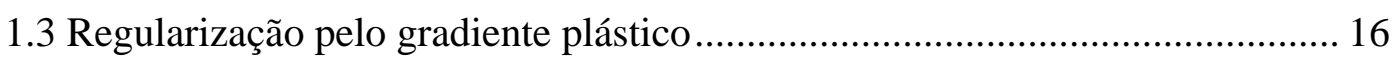

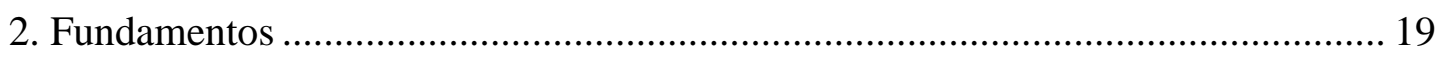

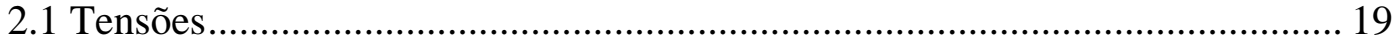

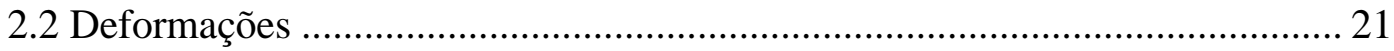

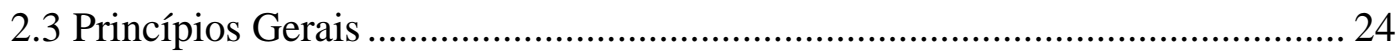

2.3.1 Equilíbrio, transformação de integrais ................................................... 24

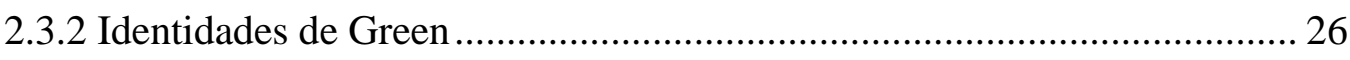

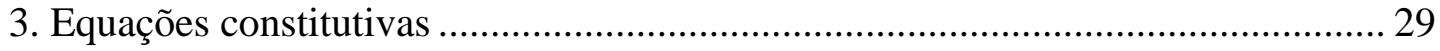

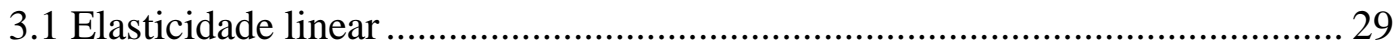

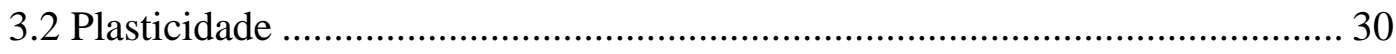

3.3 Localização de deformações ...................................................................... 33

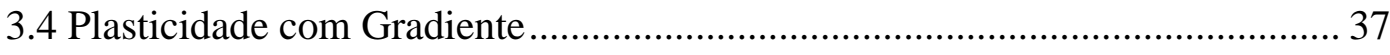

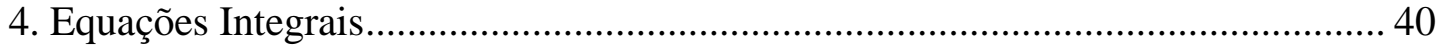

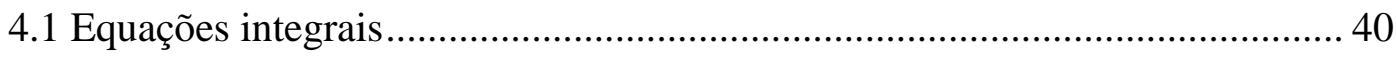

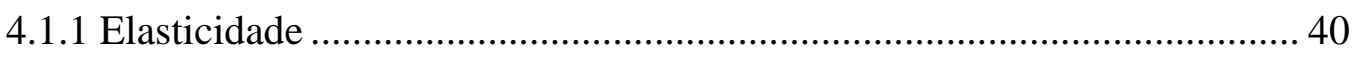

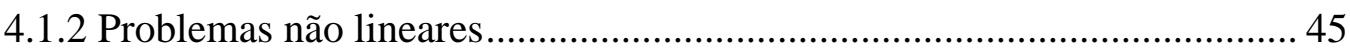

4.1.3 Representação integral de tensão no contorno ......................................... 47

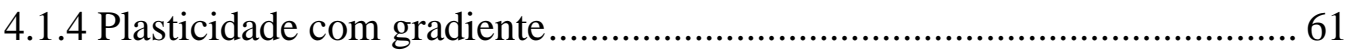


5. Método dos elementos de contorno. 66

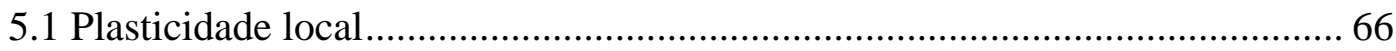

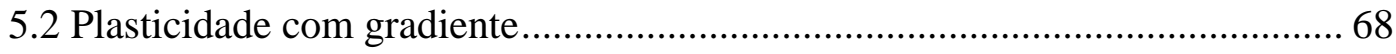

5.2.1 Esquemas de solução e condições de contorno........................................ 71

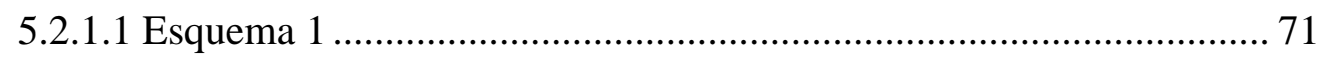

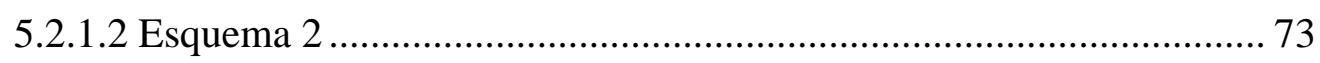

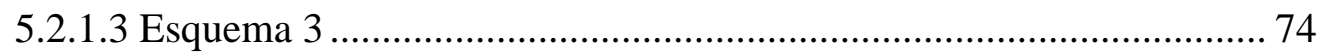

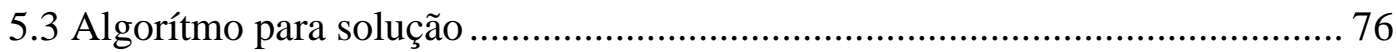

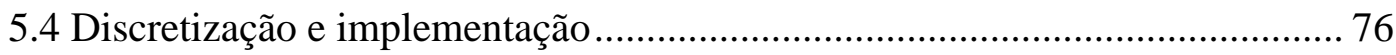

6. Formulação implícita com operador tangente consistente ................................... 80

6.1 Operador tangente consistente para o algorítmo de tensão inicial, estado plano de tensão, encruamento isótropo linear com critério de von Mises 83

6.1.1 Estado plano de tensão, algorítmo de tensão inicial............................... 87

6.1.2 Esquema do algorítmo computacional .................................................. 90

6.2 Operadores tangentes para a plasticidade com gradiente .............................. 91

6.2.1 Operador tangente consistente para a plasticidade com gradiente ........... 92

6.2.2 Operador tangente contínuo para a plasticidade com gradiente ................ 96

6.3 Uma formulação alternativa para o método dos elementos de contorno ......... 98

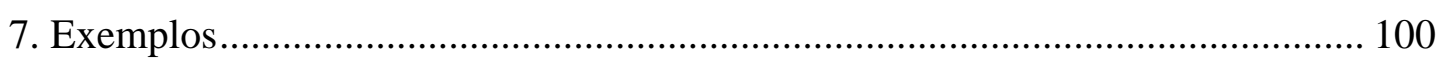

7.1 Primeira série: retângulo com enfraquecimento no canto ............................ 101

7.2 Segunda série: enfraquecimento central ................................................. 112

7.3 Terceira série: procedimentos implícitos para a plasticidade com gradiente. 125

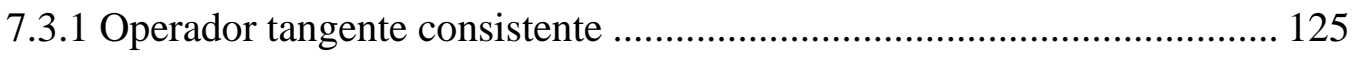

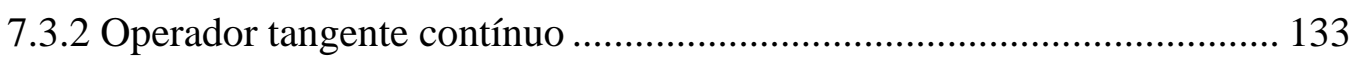

7.4 Quarta série: localização induzida por condições de contorno ....................... 136

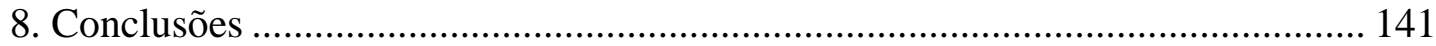

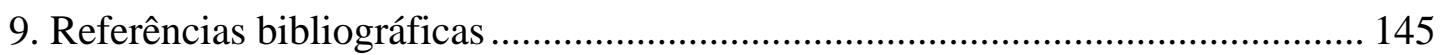




\section{LISTA DE FIGURAS}

Figura 1.1 - Relação tensão-deformação de um modelo uniaxial............................. 11

Figura 1.2 - Variação do limite plástico com a deformação plástica. ...................... 12

Figura 1.3 - Barra plástica, encruamento linear, negativo...................................... 14

Figura 4.1 - Domínio com exclusão de região contendo o ponto singular................ 42

Figura 4.2 - Região infinitesimal em torno do ponto fonte $S$................................ 50

Figura 4.3 - Elemento de contorno em coordenadas intrínsecas .............................. 53

Figura 5.1 - Solução do multiplicador plástico: esquema 1................................... 72

Figura 5.2 - Solução do multiplicador plástico: esquema 2 …................................ 74

Figura 5.3 - Solução do multiplicador plástico: esquema 3................................... 75

Figura 7.1 -- Dados para o problema do retângulo sob compressão....................... 100

Figura 7.2 - Quadrado enfraquecido no canto inferior esquerdo do retângulo. ..... 101

Figura 7.3 - Série 1: 128 células. Procedimento explícito. Plasticidade local. ...... 102

Figura 7.4 - Série 1: 128 células. Procedimento explícito. Plasticidade com

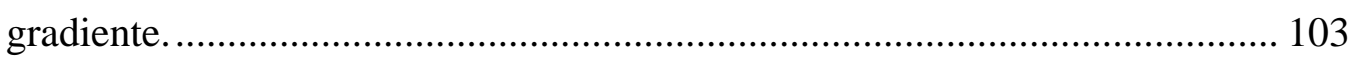

Figura 7.5 - Série 1: 128 células. Procedimento implícito. Plasticidade local....... 104

Figura 7.6 - Série 1: 512 células. Procedimento explícito. Plasticidade local. ...... 105

Figura 7.7 - Série 1: 512 células. Procedimento explícito. Plasticidade com

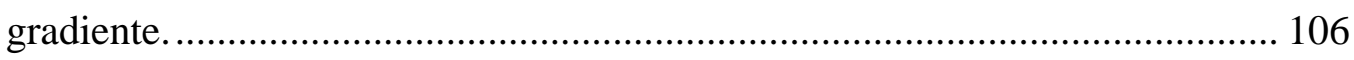

Figura 7.8 - Série 1: 512células. Procedimento implícito. Plasticidade local........ 107

Figura 7.9 - Série 1: 1024 células. Procedimento implícito. Plasticidade local..... 108

Figura 7.10 - Série 1: 2048 células. Procedimento explícito. Plasticidade local. .. 109

Figura 7.11 - Série 1: 2048 células. Procedimento explícito. Plasticidade com gradiente. 110

Figura 7.12 - Série 1: 2048 células. Procedimento implícito. Plasticidade local... 111 Figura 7.13 - Posições para os dois ou três ou um pontos centrais utilizados na segunda série.

Figura 7.14 - Série 2: 128 células. Procedimento explícito. Plasticidade local. .... 113

Figura 7.15 - Série 2: 128 células. Procedimento explícito. Plasticidade com gradiente. 
Figura 7.16 - Série 2: 128 células. Procedimento implícito. Plasticidade local..... 115

Figura 7.17 - Série 2: 512 células. Procedimento explícito. Plasticidade local. .... 116

Figura 7.18 - Série 2: 512 células. Procedimento explícito. Plasticidade com gradiente.

Figura 7.19 - Série 2: 512 células. Procedimento implícito. Plasticidade local..... 118

Figura 7.20 - Série 2: 2048 células. Procedimento explícito. Plasticidade local. .. 119

Figura 7.21 - Série 2: 2048 células. Procedimento explícito. Plasticidade com gradiente. 120

Figura 7.22 - Série 2: 2048 células. Procedimento implícito. Plasticidade local... 121

Figura 7.23 - Série 2: 2048 células. Procedimento implícito. Plasticidade local... 122

Figura 7.24 - Série 2: 512 células. Procedimento implícito. Plasticidade local..... 123

Figura 7.25 - Série 2: 256 células. Procedimento implícito. Plasticidade local..... 124

Figura 7.26 - Série 3: w=0,01 N; três pontos enfraquecidos no centro da diagonal; 2048 elementos

Figura 7.27 - Série 3: w=0,01 N; três pontos enfraquecidos no centro da diagonal; 512 elementos. Comparada com a malha de 2048 elementos (em preto) (ver Figura 7.26). 126

Figura 7.28 - Série 3: $\mathrm{w}=0,01 \mathrm{~N}$; enfraquecimento no quadrado assinalado (exceto num vértice) 126

Figura 7.29 - Série 3: w=0,01 N; enfraquecimento no quadrado assinalado (simetria).127

Figura 7.30 - Série 3: w=500 N; 2048 elementos 127

Figura 7.31 - Série 3: w=500 N; 2048 elementos (quadrado enfraquecido - menos 1 vértice) 128

Figura 7.32 - Série w=500 N; 512 elementos.................................................... 128

Figura 7.33 - Série 3: w=1000 N; 2048 elementos.............................................. 129

Figura 7.34 - Série 3: w=1000 N; 512 elementos. 129

Figura 7.35 - Série 3: w=1000 N; 2048 elementos (quadrado enfraquecido - menos 1 vértice) 130

Figura 7.36 - Série 3: w=5000 N; 2048 elementos. ............................................. 130

Figura 7.37 - Série 3: w=5000 N; 512 elementos. ................................................ 131

Figura 7.38 - Série 3: w=10000 N; 2048 elementos. 131 
Figura 7.39 - Série 3: w=10000 N; 512 elementos.

Figura 7.40 - Série 3: Plasticidade local, $\mathrm{h}=-0.025$ E.......................................... 132

Figura 7.41 - Série 3: Plasticidade com gradiente: $w=5000$ N: h=-0.025 E. .......... 133

Figura 7.42 - Série 3: w=1 N, 512 elementos, operador contínuo. ......................... 134

Figura 7.43 - Série 3: w=10000 N; 512 elementos; operador contínuo. .................. 134

Figura 7.44 - Série 3: w=500 N; 2048 elementos; operador contínuo..................... 135

Figura 7.45 - Série 3: w=5000 N; 2048 elementos; operador contínuo................... 135

Figura 7.46 - Condições de contorno do retângulo homogêneo............................... 136

Figura 7.47 - Série 4: 512 células. Procedimento explícito. Plasticidade local. .... 137

Figura 7.48 - Série 4: 512 células. Procedimento implícito. Plasticidade local..... 138

Figura 7.49 - Série 4: 512 células. Procedimento implícito. Plasticidade local..... 139

Figura 7.50 - Série 4: 2048 células. Procedimento implícito. Plasticidade local... 140 


\section{RESUMO}

O objetivo básico deste trabalho é o de estender a formulação elastoplástica do método dos elementos de contorno, de modo a incorporar a análise de corpos sujeitos ao fenômeno da localização de deformações. É utilizado um modelo de plasticidade com gradiente a fim de se evitarem as dificuldades associadas ao contínuo local. Apenas o segundo gradiente é incorporado ao modelo constitutivo. Uma representação integral para avaliação da tensão no contorno na plasticidade é obtida. O comportamento da formulação explícita clássica do método dos elementos de contorno na presença de amolecimento é analisado para a plasticidade local e com gradiente. Uma formulação implícita dos elementos de contorno utilizando o conceito de operador tangente consistente adaptado ao algorítmo de tensão inicial também é implementada e analisada. Exemplos numéricos são realizadas para mostrar a precisão dos resultados e comparar as formulações propostas. Os resultados mostram a localização de deformações ao longo de faixas estreitas determinadas pelo tamanho das células definidas pela discretização. A captura do fenômeno da localização é particularmente precisa quando se utiliza o procedimento implícito. Uma nova formulação do método dos elementos de contorno utilizando o operador tangente consistente também é proposta para a plasticidade com gradiente. 


\begin{abstract}
The basic purpose of this work is to extend the boundary element formulation to incorporate the analysis of bodies where the localization phenomena occurs. A gradient plasticity model is used in order to overcome the difficulties associated to local continua. Only the second gradient is incorporated to the constitutive model. A integral representation for boundary stress evaluation in plasticity is obtained. The behavior of the classical explicit boundary element formulation in presence of softening is analysed for local and gradient plasticity. A implicit boundary element formulation using the concept of consistent tangent operator adapted to the inicial stress agorithm is also implemented and analysed. Numerical examples are solved to show the accuracy of the results and to compare the proposed formulations. The results exhibit the strain localization along narrow bands given by the cell sizes defined by the discretization. The capture of localization phenomena is particulary accurate when the implicit scheme is used. A new implicit boundary element formulation using the consistent tangent operator is also proposed.
\end{abstract}




\section{Notação}

Matrizes:

Vetores:

Base e componentes:

Notação matricial:

Vetor transposto:

Convenção da soma:

Vetor normal unitário:

Produto escalar:

Produto vetorial:
$[A],[F],[X]$

$\mathbf{a}, \mathbf{b}, \mathbf{g}$

$\left(a_{1}, a_{2}, a_{3}\right),\left(b_{1}, b_{2}, b_{3}\right),\left(g_{1}, g_{2}, g_{3}\right)$

$\mathbf{a}=a_{1} \mathbf{i}_{1}+a_{2} \mathbf{i}_{2}+a_{3} \mathbf{i}_{3}$

$\mathbf{a}=[a]=\left(\begin{array}{lll}a_{1} & a_{2} & a_{3}\end{array}\right)$

$[a]^{T}=\left(\begin{array}{l}a_{1} \\ a_{2} \\ a_{3}\end{array}\right)$

$\mathbf{a}^{2}=a_{i} a_{i}=a_{1}^{2}+a_{2}^{2}+a_{3}^{2}$

$\hat{\mathbf{c}} \quad\left(c_{i} c_{i}=1\right)$

$\mathbf{a b}=a_{i} b_{i}=[a][b]^{T}$

$\mathbf{a} \times \mathbf{b}=\left|\begin{array}{lll}\mathbf{i}_{1} & \mathbf{i}_{2} & \mathbf{i}_{3} \\ a_{1} & a_{2} & a_{3} \\ b_{1} & b_{2} & b_{3}\end{array}\right|=\left(a_{2} b_{3}-a_{3} b_{2}\right) \mathbf{i}_{1}+$

$+\left(a_{3} b_{1}-a_{1} b_{3}\right) \mathbf{i}_{2}+\left(a_{1} b_{2}-a_{2} b_{1}\right) \mathbf{i}_{3}$ 
Delta de Kronecker: $\quad \delta_{p q}=\left\{\begin{array}{lll}1 & \text { se } & p=q \\ 0 & \text { se } & p \neq q\end{array}\right.$

Tensores: $\quad$ T, $\quad \mathbf{S}, \sigma, \varepsilon$

Componentes de tensor: $\quad T_{i j}, \varepsilon_{i j} \quad$ (segunda ordem, covariante)

Produto tensorial (definição): $(\mathbf{a} \otimes \mathbf{b}) \mathbf{c}=\mathbf{a}(\mathbf{b c})$

$$
\begin{aligned}
& (\mathbf{T} \otimes \mathbf{S}) \mathbf{R}=\mathbf{T}(\mathbf{S R}) \\
& (\mathbf{T} \otimes \mathbf{S}) \mathbf{R}=T_{i j} S_{k l} R_{k l}
\end{aligned}
$$

Produto tensorial:

$$
\mathbf{a} \otimes \mathbf{b}=\mathbf{T}
$$

$$
\mathbf{a} \otimes \mathbf{b}=[a]^{T}[b]=\left[\begin{array}{lll}
a_{1} b_{1} & a_{1} b_{2} & a_{1} b_{3} \\
a_{2} b_{1} & a_{2} b_{2} & a_{2} b_{3} \\
a_{3} b_{1} & a_{3} b_{2} & a_{3} b_{3}
\end{array}\right]
$$

$$
\mathbf{T} \otimes \mathbf{S}=T_{i j} S_{k l} \quad \text { (tensor de quarta ordem) }
$$

Base e componentes: $\quad \mathbf{T}=T_{i j} \mathbf{i}_{\mathbf{i}} \otimes \mathbf{i}_{\mathbf{j}} \quad$ (sistema cartesiano)

$$
\begin{aligned}
& \mathbf{a} \otimes \mathbf{b}=a_{i} b_{j} \mathbf{i}_{\mathbf{i}} \otimes \mathbf{i}_{\mathbf{j}} \\
& \mathbf{T} \otimes \mathbf{S}=T_{i j} S_{k l}\left(\mathbf{i}_{\mathbf{i}} \otimes \mathbf{i}_{\mathbf{j}}\right) \otimes\left(\mathbf{i}_{\mathbf{k}} \otimes \mathbf{i}_{\mathbf{l}}\right)
\end{aligned}
$$

Produto escalar de tensores: $\quad \mathbf{T S}=T_{i j} S_{i j} \quad$ (sistema cartesiano)

Traço de um tensor: $\quad \operatorname{tr}(\mathbf{T})=T_{k k}$ 
Tensor transposto: $\quad \mathbf{T}^{\mathbf{T}}=[T]^{T}=\left(\begin{array}{lll}T_{11} & T_{21} & T_{31} \\ T_{12} & T_{22} & T_{32} \\ T_{13} & T_{23} & T_{33}\end{array}\right)$

ou $\quad(\mathbf{a} \otimes \mathbf{b})^{T}=\mathbf{b} \otimes \mathbf{a}$

Outros produtos: $\quad \mathbf{a}=\mathbf{T} \mathbf{b}=\mathbf{b T}^{\mathbf{T}} \quad(\mathbf{T b}=\mathbf{b} \mathbf{T}$ se $\mathbf{T}$ é simétrico $)$

$$
\begin{array}{ll}
\text { ou: } & {[a]=[T][b]^{T}=\left([b][T]^{T}\right)^{T}} \\
\text { ou: } \quad a_{i}=T_{i j} b_{j} \quad \text { (transformação linear) }
\end{array}
$$

Tensor de $4^{\mathrm{a}}$ ordem: $\quad \mathbf{C T}=C_{i j k l} T_{k l}$

Derivada no tempo: $\quad \dot{\mathbf{a}}=\frac{d a_{k}}{d t} \mathbf{i}_{\mathbf{k}}=\dot{a}_{k} \mathbf{i}_{\mathbf{k}}$

Derivada: $\quad r_{, i}=\partial r / \partial x_{i}$

Operador $\nabla$ :

$$
\nabla=\frac{\partial}{\partial x_{k}} \mathbf{i}_{\mathbf{k}}=\partial_{k} \mathbf{i}_{\mathbf{k}} \quad \text { (como um vetor) }
$$

Laplaciano:

$$
\nabla^{2}=\nabla \nabla=\frac{\partial^{2}}{\partial^{2} x_{k}}=\partial_{1}^{2}+\partial_{2}^{2}+\partial_{3}^{2} \quad \text { (“escalar") }
$$

Gradiente de (campo) escalar: $\nabla F=\frac{\partial F}{\partial x_{k}} \mathbf{i}_{\mathbf{k}}=F_{, k} \mathbf{i}_{\mathbf{k}}=\operatorname{grad} F$

Laplaciano de um escalar: $\quad \nabla^{2} F=F_{, k k}$ 
Divergente de um vetor: $\quad \operatorname{div} \mathbf{a}=\nabla \mathbf{a}=[\nabla][a]^{T}=a_{i, i}$

Gradiente de um vetor: $\quad \operatorname{grad} \mathbf{b}=\mathbf{b} \otimes \nabla=[b]^{T}[\nabla]=b_{i, j}$

$$
\text { ou: } \quad \mathbf{b} \otimes \nabla=\left[\begin{array}{lll}
\frac{\partial b_{1}}{\partial x_{1}} & \frac{\partial b_{1}}{\partial x_{2}} & \frac{\partial b_{1}}{\partial x_{3}} \\
\frac{\partial b_{2}}{\partial x_{1}} & \frac{\partial b_{2}}{\partial x_{2}} & \frac{\partial b_{2}}{\partial x_{3}} \\
\frac{\partial b_{3}}{\partial x_{1}} & \frac{\partial b_{3}}{\partial x_{2}} & \frac{\partial b_{3}}{\partial x_{3}}
\end{array}\right] \quad \text { (jacobiano) }
$$

Transposto de $(\mathbf{b} \otimes \nabla): \quad(\mathbf{b} \otimes \nabla)^{\mathbf{T}}=\nabla \otimes \mathbf{b}$

Divergente de um tensor: $\quad \operatorname{div} \mathbf{T}=\nabla \mathbf{T}=[\nabla][T]=\frac{\partial T_{i j}}{\partial x_{i}} \mathbf{i}_{\mathbf{j}}=\partial_{i} T_{i j} \mathbf{i}_{\mathbf{j}}$

$$
\mathbf{T} \nabla=\nabla \mathbf{T}^{\mathbf{T}}=[\nabla][T]^{T}=\frac{\partial T_{i j}}{\partial x_{j}} \mathbf{i}_{\mathbf{i}}=\partial{ }_{j} T_{i j} \mathbf{i}_{\mathbf{i}}
$$




\section{Introdução}

A primeira seção deste capítulo traz uma breve descrição do tema, do trabalho realizado, as metas e os limites estabelecidos. Na segunda e terceira seções utiliza-se um modelo simples, uniaxial, para ilustrar o problema da localização de deformações na teoria do contínuo clássico e a regularização pelo gradiente plástico.

\subsection{Descrição sumária e metas}

Seguem uma pequena descrição do tema e da utilização dos elementos de contorno neste contexto, a definição dos objetivos do trabalho e seus limites, bem como uma descrição do presente texto.

\subsubsection{Localização de deformações}

Ao se observar o comportamento mecânico dos materiais ao nível macroscópico freqüentemente verifica-se a ocorrência de concentração de deformações em pequenas regiões. Nesses casos, nota-se que toda a deformação do material ocorre em pequenas zonas, faixas estreitas de localização, enquanto que nas demais partes do material verifica-se um descarregamento. As causas desse fenômeno podem estar relacionadas tanto com a geometria (como condições ou forma do corpo), quanto com características do material (tais como heterogeneidade ou pequenos defeitos). A largura das faixas de localização e suas direções depende de fatores como as condições geométricas, os parâmetros de material, as condições de carregamento. 
A origem da localização, no entanto, deve ser procurada no nível microscópico, onde heterogeneidades no material produzem um comportamento fortemente não linear (deslisamento e rotação de microestruturas, formação e crescimento de minúsculos vazios, propagação de microfissuras existentes e formação de novas), resultando num enfraquecimento naquela região, uma instabilidade local, percebida macroscopicamente como um amolecimento.

Quando a ordem de grandeza das deformações é suficientemente pequena e distribuída regularmente, a escala característica das deformações é muito maior que a das heterogeneidades do material. Nesse caso, os modelos constituivos clássicos, ou locais, expressos em alguma forma de tensão e deformação médias (equivalentes) podem ser formulados na descrição do contínuo. Na presença de amolecimento, no entanto, esse pressuposto de que a escala característica das deformações é muito maior que a das heterogeneidades não é mais apropriado. $\mathrm{O}$ tamanho da microestutura com suas heterogeneidades torna-se comparável à escala de deformações, os movimentos relativos nas microestruturas passam a contribuir significativamente para a deformação do corpo. A estabilidade material e estrutural torna-se comprometida. Matematicamente, além da perda de unicidade da solução, o problema de valor de contorno torna-se mal posicionado, com perda da elipticidade das equações diferenciais parciais que governam sua solução. Na solução numérica, esses problemas traduzem-se numa grande dependência dos resultados em relação à discretização utilizada e dos dados para a integração no tempo (processo incremental).

A localização de deformações ocorre numa grande variedade de materiais, sejam dúteis ou frágeis (metais, sintéticos, solos, rochas, concreto, cerâmicos), de modo que a existência de modelos constitutivos mais adequados seria bastante importante. Diversas alternativas tem sido propostas ao contínuo local - entre elas a que se apresenta neste trabalho, na qual a plasticidade local é enriquecida com a introdução do segundo gradiente na função de plastificação. 


\subsubsection{Método dos elementos de contorno}

O método dos elementos de contorno constitui uma alternativa importante no conjunto das técnicas numéricas mais utilizadas, como o método dos elementos finitos ou o das diferenças finitas. Em relação a esses últimos, o método dos elementos de contorno possui duas vantagens principais. Uma delas é a da possibilidade de redução de uma unidade na dimensionalidade do problema. No entanto, essa redução de uma forma completa nem sempre é simples, sendo em muitos casos problemática para certos problemas ou termos das equações governantes. A segunda vantagem parece ser mais importante e inequívoca: as equações integrais que servem de base para o método dos elementos de contorno partem de uma representação exata das quantidades físicas do problema - ao contrário do que ocorre com os elementos finitos ou diferenças finitas. É verdade porém que essa vantagem não é obtida gratuitamente, e um preço deve ser pago no tratamento das singularidades das equações integrais básicas dos elementos de contorno. Essas singularidades nas equações integrais certamente constituíram-se num dos principais obstáculos ao desenvolvimento do método dos elementos de contorno no mesmo passo que o alcançado pelos elementos finitos. Mas são justamente essas dificuldades que se encontram imbricadas às possibilidades de se obterem soluções aproximadas relativamente melhores.

Todavia, essa potencialidade teórica de se obter resultados numericamente superiores é bem distinta da disponibilidade de algorítmos de efetiva superioridade relativa. No campo da plasticidade, por exemplo, algorítmos de resolução pelo método dos elementos de contorno surgiram depois do desenvolvimento de algorítmos similares para elementos finitos (cf. TELLES (1983) [54] $^{[5}$ VENTURINI(1983) ${ }^{[58]}$, como exemplos pioneiros). Além disso, o número de trabalhos nesse campo utilizando-se elementos de contorno ainda é muito inferior ao volume de trabalho em elementos finitos no mesmo domínio. Desse modo, apesar das potencialidades previstas para o método, esforços relativamente pouco extensos foram dedicados aos elementos de contorno, por exemplo em plasticidade. Também o estudo da localização de deformações, que tem recebido recentemente grande 
atenção na mecânica aplicada (incluindo os modelos com o segundo gradiente), no que diz respeito ao aspecto das simulações numéricas, situa-se no contexto dos elementos finitos. Dentro do contexto do método dos elementos de contorno a regularização da localização de deformações aparentementemente ainda não foi computacionalmente estudada, e mesmo sua simples constatação explícita quase nem foi assinalada. (Além de FUDOLI, VENTURINI, BENALLAL [26], [27], MAIER et al. [39] parece ser a única exceção, demonstrando a localização de deformações através de uma formulação por elementos de contorno diferente da convencional - e tensão constante nas células. A regularização através do gradiente plástico não foi computacionalmente implementada no trabalho de Maier, apesar de ter sido proposta uma formulação nesse sentido.)

A utilização de formulações implícitas para a integração em problemas não lineares é outra classe de problemas que recebeu relativamente pouca atenção no contexto do método dos elementos de contorno. Em particular, formulações utilizando o conceito de operador tangente consistente tem sido utilizadas em elementos finitos a partir do trabalho de SIMO e TAYLOR $(1985)^{[51]}$, enquanto que a primeira implementação desse conceito em elementos de contorno é bem mais recente (BONNET e MUKHERJEE (1996) ${ }^{[9]}$ ) e ainda precisa ser repetida em mais implementações.

Outros aspectos relativos aos elementos de contorno, no entanto, têm sido intensamente estudados. Um exemplo é o das singularidades, que tem recebido bastante atenção, especialmente nesta década, inclusive com resultados muito recentes bem significativos. (ver, por exemplo, GUIGGIANI et al. [32], RICHARDSON $^{[49]}$.)

\subsubsection{Objetivos e delimitação}

Os objetivos iniciais do trabalho concentravam-se na formulação e análise da regularização de soluções em problemas de amolecimento, que envolvem localização de deformações. 
Logo no início do trabalho foi necessário alterar a ênfase, que passou a ser a de obtenção de uma formulação de elementos de contorno, partindo-se da formulação tradicional, que pudesse tratar adequadamente com aquela classe de problemas. A formulação convencional mostrou ter certas limitações.

Diversos foram os procedimentos implementados para se melhorar os resultados obtidos. Tanto ao nível da formulação quanto (e sobretudo) de implementação. Por exemplo, o esquema de integração utilizando-se subelementos quando o ponto de integração encontra-se próximo do ponto singular.

Destaque deve ser dado para o desenvolvimento de uma formulação utilizando-se representação integral da tensão no contorno, o que ainda não havia sido feito no caso da plasticidade ou não linearidade das equações constitutivas em geral.

Outro ponto especialmente em destaque é o desenvolvimento de formulações implícitas para a solução do problema não linear, em particular utilizando-se o conceito operador tangente consistente, que é o caso aqui apresentado, que neste trabalho foi estendida para atender ao algorítmo de tensão inicial.

Em vista das dificuldades encontradas no desenvolvimento de uma formulação eficiente para lidar com a localização de deformações, e a conseqüente mudança de ênfase dos objetivos, o problema da regularização pelo gradiente introduzido no critério de plastificação foi estabelecido sob certas particularidades. Tratam-se de simplificações, sem prejuízo da generalidade das formulações, bem entendido. Por este critério, as implementações numéricas restrigiram-se à plasticidade sob pequenas deformações, local e com gradiente, com encruamento isótropo linear e critério de von Mises.

Foi implementado um programa computacional para o caso plano de tensão, utilizando-se o algorítmo de tensão inicial. Quanto à discretização, utilizou-se interpolação linear para todas as variáveis dos problemas tratados, o que é bastante razoável.

Os exemplos numéricos tiveram como objetivo mostrar a adequação da formulação do método dos elementos de contorno para problemas de localização de 
deformações, e mostrar a possibilidade de se resolver essas dificuldades com recursos como o modelo da plasticidade com gradiente.

\subsubsection{Descrição do texto}

Após esta introdução geral, o capítulo 2 reve os fundamentos da mecânica do contínuo e alguns resultados e princípios gerais importantes para a teoria.

No capítulo 3 são apresentadas as equações constitutivas, incluindo a elasticidade linear, a plasticidade clássica, a plasticidade com gradiente. A localização de deformações também é apresentada naquele ponto.

O capítulo 4 introduz as equações integrais, ponto de partida do método dos elementos de contorno, que por sua vez é discutido no capítulo 5, incluindo uma discussão da obtenção da representação integral da tensão no contorno. No capítulo 6 é detalhada formulação implícita utilizando-se o conceito de operador tangente consistente, para a solução do problema da plasticidade através dos elementos de contorno. Neste capítulo é incluída também uma nova aplicação dessa formulação para o caso da plasticidade com gradiente, formulação esta que é apresentada sem ter sido ainda implementada.

No Capítulo 7 são apresentados os exemplos. No caso da formulação explícita convencional os resultados são razoavelmente satisfatórios, chegando a capturar a localização de deformações em alguns casos, tímida porém claramente, e mostrando o efeito da introdução do gradiente na função de plastificação. Os exemplos também evidenciam o desempenho superior da formulação implícita para solução do problema não linear, exibindo uma comparação sistemática com a obtida pelo método explícito convencional. Os exemplos com a formulação implícita capturam a localização de maneira extremamente clara. Exemplos para a plasticidade com gradiente com procedimentos implícitos também são apresentados. 


\subsection{O problema da localização de deformações}

A utilização de um modelo elementar constitui uma maneira simples de ilustrar o problema da localização no contínuo clássico (PAMIN (1994) ${ }^{[44]}$, de BORST e MULHAUS (1992) ${ }^{[12]}$, BILLARDON ${ }^{[8]}$ ).

O modelo plástico uniaxial proposto abaixo permite fazer várias considerações emblemáticas do problema localização de deformações. Na Figura 1.1, $\sigma_{\mathbf{y}_{0}}$ corresponde ao maior valor de tensão alcançado pelo material, representa limite plástico inicial, a partir do qual passa a ocorrer deformação plástica - parte descendente da curva tensão-deformação.

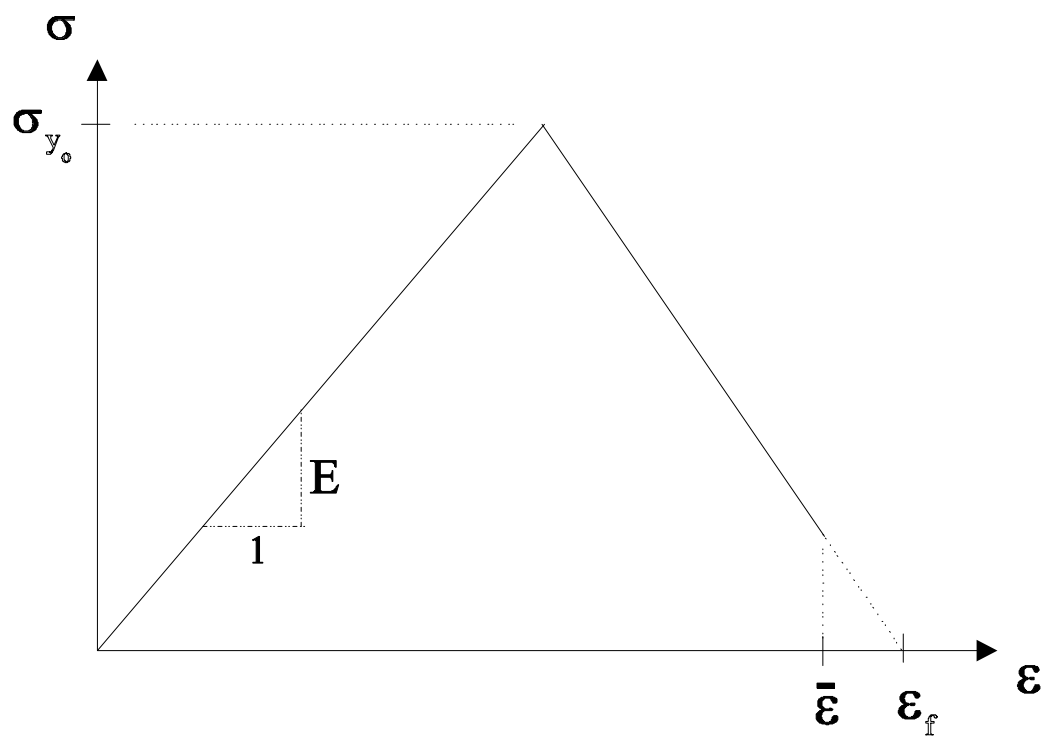

Figura 1.1 - Relação tensão-deformação de um modelo uniaxial.

$\bar{\varepsilon}$ representa a deformação máxima (ou de ruptura) do material.

A tensão não pode nunca ultrapassar o limite plástico $\sigma_{\mathbf{y}}$ (Figura 1.2), o que é expresso pela condição de plastificação:

$$
\mathbf{f}=\sigma-\sigma_{\mathbf{y}} \leq \mathbf{0}
$$


com

$$
\sigma_{\mathbf{y}}=\sigma_{\mathbf{y}_{0}}+\mathbf{h} \varepsilon^{\mathbf{p}}
$$

sendo h o módulo de deformação plástica:

$$
\mathbf{h}=-\frac{\sigma_{\mathbf{y}}}{\varepsilon_{\mathrm{f}}} .
$$

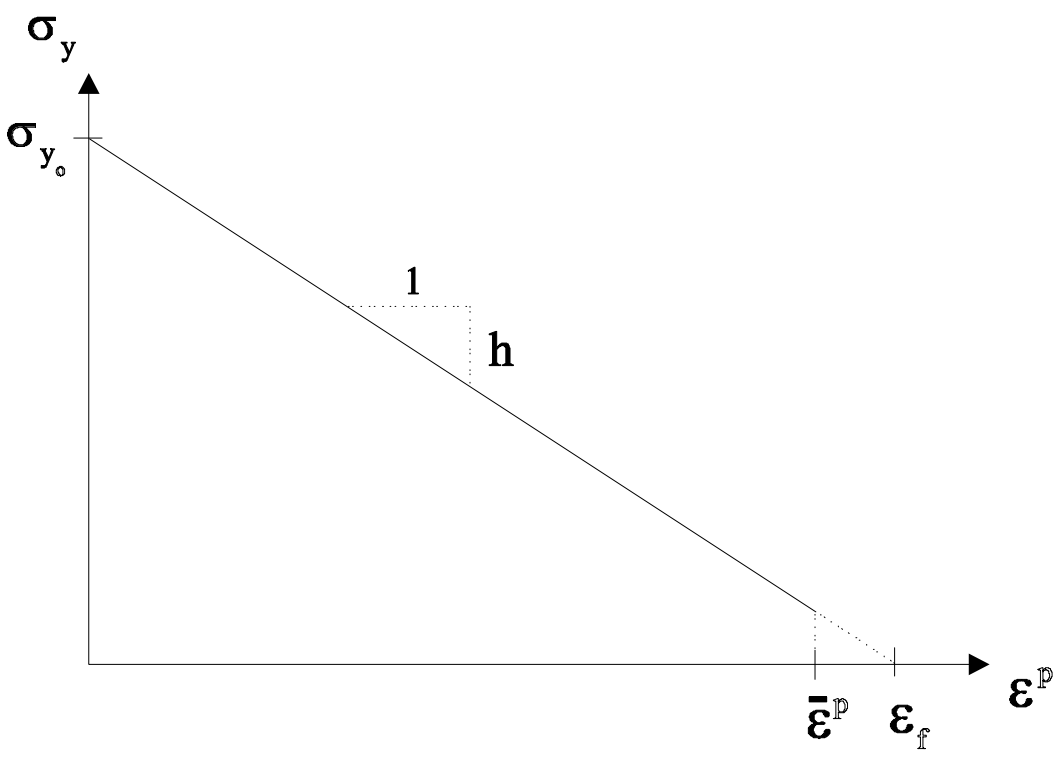

Figura 1.2 - Variação do limite plástico com a deformação plástica.

A deformação total é dada pela soma das parcelas elástica e plástica: 


$$
\varepsilon=\varepsilon^{\mathrm{e}}+\varepsilon^{\mathbf{p}}
$$

sendo a deformação elástica dada pela lei de Hooke:

$$
\varepsilon^{\mathrm{e}}=\frac{\sigma}{\mathrm{E}}
$$

onde E é o módulo de deformação elástico. A parcela plástica restante vem da relação (1.2):

$$
\varepsilon^{\mathbf{p}}=\frac{\sigma-\sigma_{\mathbf{y}_{0}}}{\mathbf{h}}
$$

Na parte elástica, $\varepsilon^{\mathbf{p}}=\mathbf{0}$, e a relação tensão-deformação em taxas pode ser diretamente obtida pela diferenciação de ( 1.5 ) em relação ao tempo, enquanto para a parte plástica, essa relação tangencial deve considerar também as equações ( 1.4 ) e ( 1.6 ). Considerando-se ambos os casos, tem-se

$$
\begin{cases}\dot{\sigma}=\mathrm{E} \dot{\varepsilon} & \text { na parte elastica }\left(\varepsilon<\sigma_{\mathbf{y}_{0}} / \mathrm{E}\right) \\ \dot{\boldsymbol{\sigma}}=\frac{\mathrm{E} \mathbf{h}}{\mathrm{E}+\mathbf{h}} \dot{\boldsymbol{\varepsilon}} & \text { na parte plastica }\end{cases}
$$

Considere-se agora uma barra, engastada à esquerda e sujeita a um deslocamento u na extremidade direita, cujo material satisfaz ao modelo plástico descrito acima (Figura 1.3). $\mathbf{x}$ refere-se ao eixo de coordenada. 


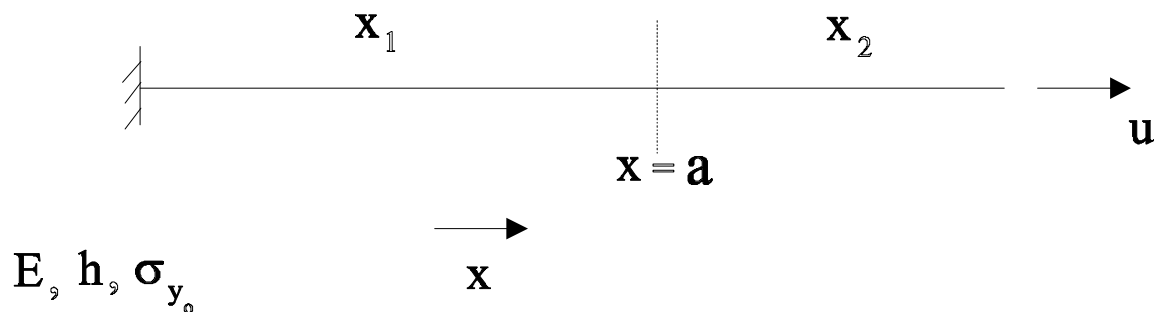

Figura 1.3 - Barra plástica, encruamento linear, negativo.

O problema é regido pelo sistema abaixo:

$$
\begin{gathered}
\dot{\mathbf{u}}=\dot{\boldsymbol{\varepsilon}}_{1} \mathbf{x}_{1}+\dot{\boldsymbol{\varepsilon}}_{2} \mathbf{x}_{2} \\
\dot{\boldsymbol{\sigma}}_{1}=\dot{\sigma}_{2} .
\end{gathered}
$$

Na parte elástica da curva tensão-deformação, ( 1.7 ), a única solução é simplesmente $\mathbf{E} \dot{\boldsymbol{\varepsilon}}_{1}=\mathbf{E} \dot{\boldsymbol{\varepsilon}}_{2}$. Na parte plástica, porém, também é possível a solução

$$
\mathbf{E} \dot{\varepsilon}_{1}=\frac{\mathbf{E h}}{\mathbf{E}+\mathbf{h}} \dot{\boldsymbol{\varepsilon}}_{2}
$$

que expressa uma situação em que ocorre carregamento com plastificação no trecho $\mathbf{x}_{\mathbf{2}}$, enquanto no trecho $\mathbf{x}_{\mathbf{1}}$ ocorre descarregamento elástico $\left(\dot{\varepsilon}_{1}<\mathbf{0}\right)$.

Outra solução corresponderia à situação inversa, com carregamento no trecho $\mathbf{x}_{1}$ e descarregamento em $\mathbf{x}_{2}$.

Em resumo, para uma mesma taxa de deslocamento imposta na extremidade livre da barra, após atingida a parte descendente da relação tensão-deformação, a solução não é única .

Mas além da perda de unicidade de solução, há um segundo aspecto de grande importância no ramo descendente da relação constitutiva: a possibilidade de 
descontinuidade de taxas de deformação. Em qualquer instante do ramo descendente, plástico, a vizinhança à esquerda de um ponto plastificado da barra pode estar em descarregamento elástico, enquanto sua vizinhança à direita está em carregamento plástico - ou o inverso. Em tal circunstância, no ponto a (Figura 1.3) haveria uma descontinuidade de deformação.

O fenômeno macroscópico da localização de deformações tem sido interpretado como a formação desse tipo de descontinuidade. Ainda com respeito ao caso uniaxial, haveria dois pontos com a condição de descontinuidade de deformações entre os quais se concentraria toda a deformação plástica da barra - com a correspondente dissipação de energia de plastificação.

A dificuldade, no contínuo clássico, ou local, é que não há definição da distância entre esses dois pontos que satisfazem a condição de descontinuidade. Dessa forma, os dois pontos devem coincidir - o que corresponde à configuração crítica. A consequiência dessa coincidência dos dois pontos é que haveria a perda total da resistência do material sem nenhuma dissipação de energia, com a plastificação ocorrendo em um trecho de comprimento nulo.

Na simulação numérica, esse comprimento fica determinado pelo tamanho da malha, que corresponde à menor distância na estrutura discretizada. Quanto mais refinada a malha, menor o comprimento de dissipação de energia. Da mesma forma que o tamanho do domínio com localização de deformações busca o comprimento nulo, na estrutura discretizada a localização procura o tamanho mínimo, definido pelo tamanho da malha. Desse modo, os resultados variam de acordo com a discretização. Quanto mais refinada a malha, menor a área de dissipação de energia, e os estados de tensão e deformação ficam igualmente dependentes. Com o refinamento da malha não há convergência para um resultado definido - pelo contrário. Além disso, a situação de instabilidade e multiplicidade de soluções, a própria convergência do procedimento numérico fica prejudicada. 


\subsection{Regularização pelo gradiente plástico}

Diversas soluções tem sido propostas para eliminar esse problema dos modelos constitutivos como a plasticidade com encruamento negativo ou a mecânica do dano - isto é, dos modelos com amolecimento do material, em geral.

O objetivo deste trabalho volta-se antes para a análise desse problema no contexto do método dos elementos de contorno que uma avaliação mais detalhada da regularização das soluções. Nesse sentido, procurou-se desenvolver uma formulação d os elementos de contorno para a plasticidade com gradiente, que constitui uma das alternativas para lidar com o problema da localização, e que recentemente tem sido bastante explorada no contexto do método dos elementos finitos.

O mesmo modelo uniaxial anterior pode ser utilizado para ilustrar o efeito regularizador do gradiente plástico no problema da localização de deformações. O gradiente plástico introduz um comprimento característico ao material, definindo assim uma largura dentro da qual a localização ocorre.

A plasticidade com gradiente é obtida introduzindo-se o segundo gradiente na função de plastificação. No caso uniaxial, o critério de plastificação ( 1.1 ) assume uma nova definição para $\sigma_{\mathbf{y}}$, no lugar de (1.2):

$$
\sigma_{\mathbf{y}}=\sigma_{\mathbf{y}_{0}}+\mathbf{h} \varepsilon^{\mathbf{p}}-\mathbf{w} \frac{d^{2} \varepsilon^{\mathbf{p}}}{d x^{2}}
$$

A fim de se analisar melhor o efeito da introdução do termo do gradiente, considere-se a solução da equação ( 1.10 ),

$$
\varepsilon^{p}=\frac{\sigma-\sigma_{y_{0}}}{h}+B \cos \frac{x}{1}
$$


sendo B uma constante de integração, $x$ refere-se aos pontos do intervalo plástico da barra, e $\mathbf{l}$ define o comprimento característico, o qual controla o tamanho do intervalo plástico na barra, e é dado por

$$
\mathbf{l}=\sqrt{-\frac{\mathbf{w}}{\mathbf{h}}}
$$

Observe-se que o sinal de $\mathbf{w}$ deve ser positivo no caso considerado, de encruamento negativo - e seria negativo para encruamento positivo. (Elastoplasticidade perfeita (h=0) não seria contemplada.) (Uma constante de sinal comum aos dois casos poderia ser definida no lugar de w.)

Utilizando-se o fato de que, durante a plastificação, $\dot{\mathbf{f}}=\mathbf{0}$ (para que a condição ( 1.1 ) seja satisfeita), pode-se derivar ( 1.11 ) em relação ao tempo, e, a seguir, aplicando-se a condição de contorno $\dot{\boldsymbol{\varepsilon}}^{\mathbf{p}}=\mathbf{0}$, a constante $\dot{\mathbf{B}}$ pode ser determinada, resultando

$$
\dot{\varepsilon}^{\mathrm{p}}=\frac{\dot{\sigma}}{\mathbf{h}}\left(1-\frac{\cos \frac{\mathrm{x}}{\mathrm{l}}}{\cos \frac{\mathrm{v}}{2 \mathrm{l}}}\right),
$$

onde $\mathbf{v}$ representa o comprimento da parte plástica da barra. A taxa de deformação total é obtida pela soma das parcelas elástica e plástica da deformação em taxas, como feito anteriormente para o caso local. O comprimento plástico $\mathbf{v}$ não é unicamente determinado, mas o caso de maior interesse é o de equilíbrio mais crítico, ou seja, o que resulta na maior taxa de deslocamento na extremidade direita da barra, u - que é obtido por integração da taxa de deformação total ao longo da barra. Assim, a condição de maximização de $\dot{\mathbf{u}}$, em relação a $\mathbf{v}$ permite a obtenção do tamanho do trecho plástico no caso mais crítico. 
O mais importante a notar, no entanto, é que o problema todo passa a ser controlado pelo comprimento característico, que é assim introduzido na descrição do contínuo, determinando um comprimento finito para a parte da barra em que ocorre plastificação. Observe-se também que a taxa de deformação plástica, definida a partir de ( 1.11 ), é contínua em todo o trecho plástico (e nula nas extremidades desse trecho), de forma que não é mais possível haver carregamento de um lado da vizinhança de um ponto e descarregamento na do outro. Esse contínuo obtido pela introdução do gradiente plástico é dito não local porque a deformação no trecho localizado é determinada para todos os pontos da região, que nesse sentido dependem uns dos outros, pela relação da deformação plástica, ao contrário da descrição clássica, em que a deformação plástica é definida para cada ponto independentemente. 


\section{Fundamentos}

\subsection{Tensões}

Seja $\sigma$ o tensor tensão num ponto, e $\hat{\mathbf{n}}$ o vetor normal a um plano que passa por esse ponto, e a composição entre eles, representando o vetor tensão, no limite a força de superfície $\mathbf{t}$ no ponto, na direção desse plano:

$$
\mathbf{t}=\sigma \hat{\mathbf{n}} \quad \text { ou } \quad t_{i}=\sigma_{i j} n_{j} .
$$

Se $\hat{\mathbf{m}}$ representa a direção de um plano de tensão principal,

$$
\sigma \hat{\mathbf{m}}=\alpha \hat{\mathbf{m}}
$$

onde $\alpha$ representa a tensão principal na direção de $\hat{\mathbf{m}}$. Ou, com notação indicial,

$$
\left(\sigma_{i j}-\alpha \delta_{i j}\right) m_{i}=0
$$

que possui solução não trivial se o determinante dos coeficientes for nulo:

$$
\operatorname{det}\left[\sigma_{i j}-\alpha \delta_{i j}\right]=0
$$


A solução da equação de terceiro grau resultante dessa condição fornece os valores das três direções principais, cujas direções podem ser obtidas de ( 2.3 ), lembrando-se que

$$
m_{i} m_{i}=1 .
$$

A equação cúbica do determinante ( 2.3 ) pode ser expressa como

$$
\alpha^{3}-I_{1} \alpha^{2}-I_{2} \alpha-I_{3}=0,
$$

onde $I_{1}, I_{2}, I_{3}$ são os invariantes de tensão:

$$
\begin{gathered}
I_{1}=\sigma_{11}+\sigma_{12}+\sigma_{13}=\sigma_{k k}=\operatorname{tr}(\sigma), \\
I_{2}=\sigma_{11} \sigma_{22}+\sigma_{22} \sigma_{33}+\sigma_{33} \sigma_{11}-\sigma_{23}^{2}-\sigma_{31}^{2}-\sigma_{12}^{2}= \\
=\frac{1}{2}\left(\sigma_{i i} \sigma_{j j}-\sigma_{i j} \sigma_{i j}\right)=\frac{1}{2}\left(I_{1}^{2}-\sigma_{i j} \sigma_{i j}\right)=\frac{1}{2}\left(\operatorname{tr}^{2}(\sigma)-\sigma \sigma\right), \\
I_{3}=\operatorname{det} \sigma=\left|\begin{array}{lll}
\sigma_{11} & \sigma_{12} & \sigma_{13} \\
\sigma_{21} & \sigma_{22} & \sigma_{23} \\
\sigma_{31} & \sigma_{32} & \sigma_{33}
\end{array}\right| .
\end{gathered}
$$

Um tensor de tensão $\sigma$ pode ser decomposto em uma parcela hidrostática (ou esférica) e outra desviadora. O tensor desviador expressa-se como

$$
\mathbf{S}=\sigma-\sigma_{m} \mathbf{I}
$$

onde, I é o tensor identidade (de segunda ordem), e o tensor hidrostático $\sigma_{m}$ é dado por 


$$
\sigma_{m}=\frac{1}{3} I_{1}=\frac{1}{3} \operatorname{tr}(\sigma) .
$$

Os invariantes do tensor desviador então resultam em

$$
\begin{gathered}
J_{1}=0, \\
\left.J_{2}=\frac{1}{6}\left[\sigma_{11}-\sigma_{22}\right)^{2}+\left(\sigma_{22}-\sigma_{33}\right)^{2}+\left(\sigma_{33}-\sigma_{11}\right)^{2}\right]+ \\
+\sigma_{12}^{2}+\sigma_{23}^{2}+\sigma_{13}^{2}=\frac{I_{1}^{2}}{3}-I_{2}, \\
J_{3}=\frac{2 I_{1}^{3}}{27}-\frac{I_{1} I_{2}}{3}+I_{3} .
\end{gathered}
$$

Um estado plano de tensão define-se por

$$
\begin{gathered}
\sigma_{33}=0, \sigma_{13}=\sigma_{31}=0, \sigma_{23}=\sigma_{32}=0, \\
\text { ou seja, } \\
{[\sigma]=\left[\begin{array}{ccc}
\sigma_{11} & \sigma_{12} & 0 \\
\sigma_{21} & \sigma_{22} & 0 \\
0 & 0 & 0
\end{array}\right] .}
\end{gathered}
$$

\subsection{Deformações}

Sendo $d S$ a distância entre dois pontos de material em seu estado inicial e $d \mathbf{u}$ o deslocamento relativo ocorrido entre eles num tempo t, e assumindo-se pequenas 
deformações (e deslocamentos), o deslocamento relativo unitário $d \mathbf{u} / d S$ expressa-se por

$$
\frac{d \mathbf{u}}{d S}=(\mathbf{u} \otimes \nabla) \hat{\mathbf{n}}=\hat{\mathbf{n}}(\nabla \otimes \mathbf{u}),
$$

onde $\hat{\mathbf{n}}$ representa o vetor normal unitário (de componentes $d x_{i} / d S$ ) na direção da distância $d S$ entre os pontos. Em notação indicial:

$$
\frac{d u_{i}}{d S}=\frac{d u_{i}}{d x_{j}} \frac{d x_{j}}{d S}
$$

ou matricial:

$$
\left[\begin{array}{l}
\frac{\partial u_{1}}{\partial S} \\
\frac{\partial u_{2}}{\partial S} \\
\frac{\partial u_{3}}{\partial S}
\end{array}\right]=\left[\begin{array}{lll}
\frac{\partial u_{1}}{\partial x_{1}} & \frac{\partial u_{1}}{\partial x_{2}} & \frac{\partial u_{1}}{\partial x_{3}} \\
\frac{\partial u_{2}}{\partial x_{1}} & \frac{\partial u_{2}}{\partial x_{2}} & \frac{\partial u_{2}}{\partial x_{3}} \\
\frac{\partial u_{3}}{\partial x_{1}} & \frac{\partial u_{3}}{\partial x_{2}} & \frac{\partial u_{3}}{\partial x_{3}}
\end{array}\right]\left[\begin{array}{l}
\frac{\partial x_{1}}{\partial S} \\
\frac{\partial x_{2}}{\partial S} \\
\frac{\partial x_{3}}{\partial S}
\end{array}\right] .
$$

O jacobiano $\mathbf{u} \otimes \nabla$ pode ser divido em duas parcelas, o tensor de deformação $\varepsilon$ e o tensor de rotação (de corpo rígido) $\mathbf{R}$ :

$$
\mathbf{u} \otimes \nabla=\varepsilon+\mathbf{R}=\frac{1}{2}[\mathbf{u} \otimes \nabla+\nabla \otimes \mathbf{u}]+\frac{1}{2}[\mathbf{u} \otimes \nabla-\nabla \otimes \mathbf{u}],
$$

ou, seja, 


$$
\varepsilon=\frac{1}{2}\left[(\nabla \otimes \mathbf{u})^{T}+\nabla \otimes \mathbf{u}\right]
$$

Em notação indicial e matricial, o tensor de deformação expressa-se como

$$
\varepsilon_{i j}=\frac{1}{2}\left(\frac{\partial u_{i}}{\partial x_{j}}+\frac{\partial u_{i}}{\partial x_{j}}\right) \quad \text { ou } \quad \varepsilon=[\varepsilon]=\left[\begin{array}{lll}
\varepsilon_{11} & \frac{1}{2} \gamma_{12} & \frac{1}{2} \gamma_{12} \\
\frac{1}{2} \gamma_{12} & \varepsilon_{22} & \frac{1}{2} \gamma_{12} \\
\frac{1}{2} \gamma_{12} & \frac{1}{2} \gamma_{12} & \varepsilon \varepsilon_{33}
\end{array}\right]
$$

Direções principais de deformações e invariantes podem ser expressos de forma análoga à do tensor de tensão. Em particular, a deformação hidrostática, que expressa a dilatação ou alteração de volume, é dada por

$$
e=\operatorname{tr}(\varepsilon)
$$

Um estado plano de deformação define-se como

$$
\begin{gathered}
\varepsilon_{33}=0, \varepsilon_{13}=\varepsilon_{31}=0, \varepsilon_{23}=\varepsilon_{32}=0, \\
\text { ou seja, } \\
{[E]=\left[\begin{array}{ccc}
\varepsilon_{11} & \varepsilon_{12} & 0 \\
\varepsilon_{21} & \varepsilon_{22} & 0 \\
0 & 0 & 0
\end{array}\right] .}
\end{gathered}
$$




\subsection{Princípios Gerais}

A seguir são enumerados alguns princípios e teoremas importantes no contexto deste trabalho.

\subsubsection{Equilíbrio, transformação de integrais}

$\mathrm{Na}$ ausência de aceleração das partículas, as equações de movimento de Cauchy reduzem-se às relações de equilíbrio seguintes, muito importantes na mecânica dos sólidos:

$$
\frac{\partial \sigma_{i j}}{\partial x_{i}}+b_{j}=0 \quad \text { ou } \quad \operatorname{div} \sigma+\mathbf{b}=0
$$

onde b representa as forças volumétricas e $\sigma$ é o tensor de tensão. (O tensor $\sigma$ é aqui assumido como simétrico - caso não polar).

Para uma função escalar $f\left(x_{1}, x_{2}, x_{3}\right)$ com primeiras derivadas parciais contínuas, vale o teorema de transformação de uma integral de superfície em integral de volume (teorema de Green):

$$
\int_{S} f n_{i} d S=\int_{V} \frac{\partial f}{\partial x_{i}} d V
$$

sendo $n_{i}$ uma componente do vetor normal à superfície. A expressão anterior, aplicada às componentes de um vetor $\mathbf{v}$ conduz ao teorema da divergência (ou teorema de Gauss): 


$$
\begin{gathered}
\int_{S} v_{i} n_{i} d S=\int_{V} \frac{\partial v_{i}}{\partial x_{i}} d V \\
\text { ou } \\
\int_{S} \mathbf{v} \hat{\mathbf{n}} d S=\int_{V} \nabla \mathbf{v} d V,
\end{gathered}
$$

que possibilita expressar a integração por partes, na forma seguinte:

$$
\int_{V} \frac{\partial u_{j}}{\partial x_{i}} \sigma_{i j} d V=\int_{S} \sigma_{i j} u_{j} n_{i} d S-\int_{V} \frac{\partial \sigma_{i j}}{\partial x_{i}} u_{j} d V
$$

ou

$$
\int_{V}(\nabla \otimes \mathbf{u}) \boldsymbol{\sigma} d V=\int_{S}(\hat{\mathbf{n}} \boldsymbol{\sigma}) \mathbf{u} d S-\int_{V}(\operatorname{div} \boldsymbol{\sigma}) \mathbf{u} d V
$$

Essa expressão da integração por partes é particularmente importante, por acarretar quase imediatamente princípios fundamentais na mecânica das estruturas: o princípio dos deslocamentos (ou trabalhos) virtuais, o princípio dos esforços virtuais, que por sua vez possibilitam expressar o teorema de Betti, e podem todos ser apropriadamente apresentados a partir da primeira identidade de Green (deslocamentos ou esforços virtuais) e segunda identidade de Green (teorema de Betti).

Observe-se que a expressão ( $\mathbf{2 . 2 3}$ ) pode ser retrabalhada, utilizando-se a simetria do tensor de tensão $\sigma$, a equação de equilíbrio ( 2.20 ), o equilíbrio de superfície (eq. ( 2.1 )) e o tensor de deformação $\varepsilon$ (eq. ( 2.17 )), resultando na expressão

$$
\int_{V} \varepsilon_{i j} T_{i j} d V=\int_{S} t_{j} u_{j} d S+\int_{V} b_{j} u_{j} d V
$$


que já aparece muito próxima do princípio dos trabalhos virtuais (PTV), tão importante no contexto do método dos elementos finitos.

Partindo-se de

$$
\int_{V}(\nabla \otimes \delta \mathbf{u}) \sigma d V
$$

e utilizando-se ( 2.24 ) obtem-se o princípio dos trabalhos virtuais, em correspondência com a expressão ( 2.25 ):

$$
\int_{V} \sigma_{i j} \delta \varepsilon_{i j} d V=\int_{S} t_{j} \delta u_{j} d S+\int_{V} b_{j} \delta u_{j} d V
$$

A única exigência sobre $\delta$ u é que seja cinematicamente admissível; isto é, suas primeiras derivadas parciais sejam contínuas (classe $C^{l}$ ) e, por conveniência, tenha valor nulo onde os deslocamentos são prescritos no contorno. Observe-se que não é necessário que o campo de deslocamentos virtuais $\delta \mathbf{u}$ seja infinitesimal (contrariamente ao que se afirma algumas vezes).

\subsubsection{Identidades de Green}

Seguindo Hartmann ${ }^{[34],[35]}$, considere-se dois campos vetoriais $\mathbf{u}$ e $\mathbf{v}$ tais que

$$
\mathbf{u} \in C^{2 m} \quad e \quad \mathbf{v} \in C^{m},
$$

ou seja, u e $\mathbf{v}$ possuem derivadas parciais contínuas no domínio, de ordem $2 m$ e $m$, respectivamente, sendo $2 m$ a ordem de um operador diferencial $L$ sobre o domínio $(\Omega)$. A aplicação sucessiva da integração por partes nos termos relevantes, iniciando 
pela integral contendo o operador $L$, resulta numa expressão contendo operadores diferenciais I sobre o contorno $(\Gamma)$ (com os sobrescritos indicando sua ordem), que representa esquematicamente a primeira identidade de Green:

$$
G(\mathbf{u}, \mathbf{v})=\int_{\Omega}(\mathrm{L} \mathbf{u}) \mathbf{v} d \Omega-\sum_{i=1}^{m}(-1)^{i} \int_{\mathrm{T}}\left(\left.\right|^{2 m-i} \mathbf{u}\right)\left(\left.\right|^{i-1} \mathbf{v}\right) d \Gamma-2 E(\mathbf{u}, \mathbf{v})=0
$$

onde aparecerão termos com derivadas parciais de ordem $m$ em $E(\boldsymbol{u}, \mathbf{v})$, que são termos integrais representando a energia de deformação. Os pares com os operadores diferenciais I que aparecem em cada integral de contorno representam os conjugados, com o termo de maior ordem do operador denotando "forças" e o menor referindo-se aos "deslocamentos".

A aplicação dessa identidade será ilustrada na próxima seção para o caso da elasticidade linear, que é o de interesse no presente contexto. A expressão ( 2.29 ) resultaria então no princípio dos deslocamentos virtuais se no lugar de $\mathbf{v}$ fosse imposto um deslocamento virtual cinematicamente admissível $\delta \mathbf{u}$, reproduzindo assim a expressão ( 2.27 ).

O princípio dos trabalhos virtuais pode ser obtido diretamente de ( 2.29 ), adotando-se um deslocamento virtual estaticamente admissível $\delta \mathbf{u}$ (isto é, satisfazendo as relações de equilíbrio ( 2.20 ) e, por conveniência, satisfazendo os valores prescritos para o tensor de tensão). Ou seja,

$$
G(\delta \mathbf{u}, \mathbf{u})=0
$$

com $\delta \mathbf{u}, \mathbf{u}$ satisfazendo as condições ( 2.28 ), respectivamente.

Considere-se agora as condições 


$$
\mathbf{u}, \mathbf{v} \in C^{2 m}
$$

que permitem aplicar a primeira identidade de Green ( 2.29 ) duas vezes como segue, obtendo-se a segunda identidade de Green:

$$
\begin{gathered}
B(\mathbf{u}, \mathbf{v})=G(\mathbf{u}, \mathbf{v})-G(\mathbf{v}, \mathbf{u})= \\
=\int_{\Omega}(\mathrm{L} \mathbf{u}) \mathbf{v} d \Omega-\sum_{i=1}^{2 m}(-1)^{i} \int_{\mathrm{T}}\left(\left.\right|^{2 m-i} \mathbf{u}\right)\left(\left.\right|^{i-1} \mathbf{v}\right) d \Gamma-\int_{\Omega}(\mathrm{L} \mathbf{v}) \mathbf{u} d \Omega=0 .
\end{gathered}
$$

As integrais de domínio $E(\mathbf{u}, \mathbf{v})$ e $E(\mathbf{v}, \mathbf{u})$ (energia de deformação) nas duas aplicações de $G$ são idênticas, cancelando-se reciprocamente.

No caso dos operadores da elasticidade linear, ( 2.32 ) corresponde ao teorema de Betti.

A expressão ( 2.32 ) é o ponto de partida para a obtenção das equações integrais utilizadas pelo métodos dos elementos de contorno. Basta tomar uma solução fundamental no lugar de um dos campos vetoriais em ( 2.32 ).

Ressalte-se que as identidades de Green apresentadas acima configuram uma abordagem formalmente equivalente à técnica dos resíduos ponderados, frequentemente aplicada aos métodos numéricos, e em particular no contexto do método dos elementos finitos e no método dos elementos de contorno. 


\section{Equações constitutivas}

\subsection{Elasticidade linear}

A lei de Hooke estabelece as relações entre o tensor de tensão e o de deformação para a elasticidade linear isótropa sob pequenas deformações:

$$
\sigma=\mathbf{C} \varepsilon \quad \text { ou } \quad \sigma_{i j}=C_{i j k l} \varepsilon_{k l},
$$

onde $\mathbf{C}$ é o tensor de rigidez elástico (quarta ordem):

$$
C_{i j k l}=\lambda_{c} \delta_{i j} \delta_{k l}+\mu\left(\delta_{i k} \delta_{j l}+\delta_{i l} \delta_{j k}\right)
$$

sendo $\mu$ e $\lambda_{c}$ as constantes elásticas de Lamé (parâmetros do material).

Considerando-se ( 3.2 ) a lei de Hooke pode ser reescrita como

$$
\sigma_{i j}=\lambda_{c} \varepsilon_{k k} \delta_{i j}+2 \mu \varepsilon_{i j}
$$

O problema de valor de contorno da teoria da elasticidade pode agora ser escrito por completo, compondo-se das equações de deformação ( 2.16 ), de equilíbrio ( 2.20 ) e das relações tensão deformação ( 3.1 ): 


$$
\begin{gathered}
\varepsilon=\frac{1}{2}\left[(\nabla \otimes \mathbf{u})^{T}+\nabla \otimes \mathbf{u}\right] \\
\nabla \boldsymbol{\sigma}+\mathbf{b}=0 \\
\sigma=\mathbf{C} \varepsilon
\end{gathered}
$$

que podem ser resumidas num só conjunto, em termos do deslocamento u, substituindose umas nas outras, resultando nas equações de Navier:

$$
\begin{gathered}
(-\mathrm{L} \mathbf{u})=-\mu \nabla(\nabla \otimes \mathbf{u})-\frac{\mu}{1-2 \nu} \nabla(\nabla \mathbf{u})=\mathbf{b} \\
\text { ou } \\
\left(-\mathrm{L}_{k l} u_{l}\right)=-\mu u_{k, l l}-\frac{\mu}{1-2 v} u_{l, l k}=b_{k} .
\end{gathered}
$$

Portanto, o operador da elasticidade linear isótropa sob pequenas deformações é o que aparece na expressão anterior:

$$
L()=\mu \operatorname{div}(\operatorname{grad}())^{T}+\frac{\mu}{1-2 v} \operatorname{grad} \operatorname{div}(),
$$

com o problema de valor de contorno regido pelas equações ( 3.5 ).

\subsection{Plasticidade}

Para modelar o comportamento elastoplástico multiaxial é preciso especificar um critério de plastificação, definir a composição do tensor de deformação total e sua relação com as tensões, e estabelecer a evolução dos parâmetros de encruamento e das deformações plásticas. No modelo aqui tratado assume-se pequenas deformações (BENALLAL e TVERGAARD ${ }^{[7]}$, MUHLHAUS e AINFANTIS ${ }^{[41]}$ ). 
Como trata-se de um problema não linear, dependente do tempo (pseudotempo), é preciso considerar as taxas. Com $\dot{\boldsymbol{\varepsilon}}^{\mathbf{p}}$ representando a taxa do tensor de deformação total e $\dot{\varepsilon}^{\mathbf{p}}$ a da deformação plástica, a composição das deformações e relação com as tensões $\dot{\sigma}$ é expressa pelo incremento do tensor de tensão de Cauchy:

$$
\dot{\sigma}=\mathbf{C}\left(\dot{\varepsilon}-\dot{\varepsilon}^{\mathbf{p}}\right)
$$

sendo $\mathbf{C}$ o tensor de rigidez elástico.

O critério de plastificação é dado em função do tensor de tensão $\sigma$ e da deformação plástica acumulada $p$ :

$$
f(\mathbf{T}, R(p))=0,
$$

com $R$ representando o tamanho da superfície de plastificação e

$$
\dot{p}=\sqrt{2 \dot{\varepsilon}^{\mathrm{p}} \dot{\boldsymbol{\varepsilon}}^{\mathbf{p}}}
$$

A regra de fluxo regendo a plastificação é

$$
\dot{\varepsilon}^{\mathbf{p}}=\dot{\lambda} \frac{\partial F}{\partial \sigma}
$$

onde $\lambda^{\bullet}$ é o multiplicador plástico e $F(\sigma, R)$ denota o potencial plástico, com $(\partial F / \partial \sigma)=(\partial f / \partial \sigma)$ no caso da associatividade, aqui assumida, implicando na normalidade da regra de fluxo.

A evolução do encruamento é dada pela regra 


$$
\dot{p}=\dot{\lambda} \frac{\partial F}{\partial R}
$$

O multiplicador plástico que aparece nas duas expressões anteriores deve obedecer às condições de Kuhn-Tucker:

$$
\dot{\lambda} \geq 0, \quad f \leq 0, \quad \dot{\lambda} f=0,
$$

com $\dot{\lambda}$ podendo ser obtido da condição de consistência,

$$
\dot{f}=0,
$$

e utilizando-se as relações ( 3.7 )-( 3.11 ), obtendo-se a expressão

$$
\dot{\lambda}=\frac{\frac{\partial f}{\partial \sigma} \mathbf{C} \dot{\varepsilon}}{h+\frac{\partial f}{\partial \sigma} \mathbf{C} \frac{\partial F}{\partial \sigma}},
$$

com o módulo de encruamento $h$ sendo dado por

$$
h=-\frac{\partial f}{\partial R} \frac{\partial R}{\partial p} \frac{\partial F}{\partial R} .
$$

Resumindo, o problema de valor de contorno em taxas fica dado por ( 3.7 ), ( 3.10 ) e ( 3.8 ) sujeita às condições de ( 3.12 ) (com ( 3.13 )). 


\subsection{Localização de deformações}

No nível macroscópico pode-se observar frequentemente a concentração de deformações em pequenas regiões de um sólido, em decorrência quer de fatores geométricos (forma e condições de contorno), quer de imperfeições (heterogeneidades ou defeitos locais). Os materiais em que se pode observar a localização de deformações podem ser dúteis (plasticidade) ou frágeis (dano). $\mathrm{O}$ fenômeno está relacionado com o enfraquecimento do material, amolecimento, e pode ocorrer no caso de encruamento negativo (na plasticidade), ou com a evolução do dano (mecânica do dano).

O problema da localização é basicamente descrito a seguir (ver PAMIN ${ }^{[44]}$ )

No contexto da mecânica do contínuo clássica define-se o conceito de estabilidade material (HILL ${ }^{[36],}$ DRUCKER $^{[22]}$ ):

$$
\dot{\sigma} \dot{\varepsilon}>0
$$

com a estabilidade de um corpo definida como

$$
\int_{V} \dot{\sigma} \dot{\varepsilon} d V>0
$$

sendo que existência de pontos em que a condição ( 3.16 ) é violada pode conduzir à instabilidade estrutural.

A instabilidade material ocorre tipicamente em casos de enfraquecimento (por exemplo na região decrescente de um gráfico da relação tensão-deformação uniaxial).

Considere-se a equação constitutiva em sua forma incremental:

$$
\dot{\sigma}=\mathbf{C}^{\mathrm{eq}} \dot{\varepsilon},
$$


onde $\mathbf{C}^{\mathbf{e q}}$ é o tensor de rigidez tangente. (No caso da plasticidade associada, assumido aqui, $\mathbf{C}^{\mathbf{e q}}$ é simétrico, mas isso não ocorre em geral). Carregando-se continuamente um corpo, a condição ( 3.16 ) deixa ocorrer quando o tensor de rigidez tangente $\mathbf{C}^{\text {eq }}$ não é mais positivo definido, o que pode ser verificado quando

$$
\dot{\varepsilon} \mathbf{C}^{\mathrm{eq}} \dot{\boldsymbol{\varepsilon}} \leq \mathbf{0}, \quad(\text { com } \dot{\varepsilon} \neq \mathbf{0})
$$

Por outro lado, a situação limite da condição de estabilidade material também marca a perda de unicidade da solução:

$$
\dot{\sigma}=\mathbf{C}^{\mathrm{eq}} \dot{\boldsymbol{\varepsilon}}=0,
$$

que verifica-se quando

$$
\operatorname{det}\left[C^{e q}\right]=\left|\left[C^{e q}\right]\right|=0 \text {. }
$$

Ressalte-se que a perda de estabilidade material (condição ( 3.19 )) ocorre simultaneamente com a perda de unicidade de solução (condição ( 3.21 )) das equações constitutivas ( 3.18 ), quando o tensor de rigidez tangente é simétrico, como ocorre na plasticidade associada. Quando o tensor de rigidez tangente não é simétrico, pode ocorrer perda de estabilidade material mesmo antes da perda de unicidade.

Substituindo-se agora a equação ( 3.14 ) em ( 3.10 ) e daí em ( 3.7 ), é possível se obter $\dot{\varepsilon}=\left(\mathbf{C}^{\mathrm{eq}}\right)^{-1} \dot{\boldsymbol{\sigma}}$, e daí $\dot{\boldsymbol{\sigma}} \dot{\mathbf{T}}=\mathbf{C}^{\mathrm{eq}} \dot{\boldsymbol{\varepsilon}}$, para então analisar $\dot{\varepsilon} \mathbf{C}^{\mathrm{eq}} \dot{\boldsymbol{\varepsilon}}$, como sugere a condição ( 3.19 ). Conclui-se então que na plasticidade associada a perda de estabilidade é possível se $h$, o módulo de encruamento é negativo. Há um estado neutro, não estável e não instável, se $h=0$, que corresponde à elastoplasticidade perfeita. 
Por outro lado, considere-se agora a condição estática de localização. Admitase um corpo homogêneo, deformado também de modo homogêneo. Admita-se também que, ao receber um novo incremento de deformação, apareça um plano, de normal $\hat{\mathbf{n}}$, ao longo do qual há uma descontinuidade no gradiente do campo de deslocamentos: RUDNICK ${ }^{[50],}$ ORTIZ et al. ${ }^{[43]}$.

$$
\{\mathbf{u} \otimes \nabla\}=\left\{u_{i, j}\right\}=u_{i, j}^{+}-u_{i, j}^{-} \neq 0
$$

onde ' \{\} ' é utilizado para representar o valor do salto no gradiente de um lado para o outro do plano definido por $\hat{\mathbf{n}}$ e os sinais ' + ' e '- ' designam os valores de $u_{i, j}$ de cada lado do plano.

O valor da descontinuidade pode ser representado pela condição de Maxwell:

$$
\left\{u_{i, j}\right\}=n_{i} m_{j}
$$

onde m é um vetor arbitrário. Usando-se a expressão acima, o valor da descontinuidade no tensor de deformação fica como

$$
\left\{\varepsilon_{i j}\right\}=\frac{1}{2}\left(n_{i} m_{j}+n_{j} m_{i}\right)
$$

A equação constitutiva ( 3.18 ) no momento da formação dessa bifurcação fornece:

$$
\left\{\dot{\sigma}_{i j}\right\}=C_{i j k l}^{e q}\left\{\dot{\varepsilon}_{i j}\right\},
$$

admitindo-se que $C_{i j k l}^{e q}$ é contínuo através da descontinuidade. 
Durante a formação dessa descontinuidade o vetor de tensão $\dot{\mathfrak{t}}$ deve ser contínuo ao longo do plano definido por $\hat{\mathbf{n}}$ :

$$
0=\left\{\dot{t}_{i}\right\}=\left\{\dot{\sigma}_{i j}\right\} n_{j}=0
$$

Substituindo-se ( 3.24 ) e ( 3.25 ) em ( 3.26 ) e utilizando-se a propriedade $C_{i j k l}=C_{i j l k}$, obtem-se a condição para que ocorra a bifurcação:

$$
\left(n_{i} C_{i j k l} n_{l}\right) m_{k}=0
$$

O tensor $Q_{j k}=n_{i} C_{i j k l} n_{l}$ é denominado tensor acústico e seu determinante fornece a condição procurada:

$$
\operatorname{det}[Q]=\operatorname{det}\left(n_{i} C_{i j k l} n_{l}\right)=0 .
$$

Portanto, a solução da expressão anterior fornece $\hat{\mathbf{n}}$, a direção do plano de descontinuidade das deformações, que poderá então ser usado em ( 3.27 ) para determinar também o valor do salto de descontinuidade $\left(n_{i} m_{j}\right)$.

A singularidade do tensor acústico corresponde à perda de elipticidade das equações de equilíbrio em taxas BENALLAL et al. (1991) ${ }^{[6]}$. (Quando a equações diferenciais parciais de equilíbrio deixam de ser elípticas, significa que o problema de valor de contorno em taxas deixa de ter um número finito de soluções contínuas linearmente independentes.) (Sob pequenas deformações, só ocorre perda de elipticidade se também tiver ocorrido perda de estabilidade material (de BORST $\left.\left.(1993)^{[13]}.\right)\right)$

É importante notar ainda que a descontinuidade de deformação tem sido entendida como a responsável pela concentração de deformação observada ao nível macroscópico numa região de um material. Teoricamente, isto se descreveria como a 
região confinada entre dois planos de descontinuidades. A distância entre esses planos fica no entanto indefinida no contínuo clássico, de modo que os dois planos deveriam coincidir, configurando uma distribuição de alta deformação ao longo de uma faixa de largura nula. Em métodos numéricos, no contexto do método dos elementos finitos sobretudo, isto foi largamente observado. Sob valores negativos do módulo de encruamento, é possível formar-se uma faixa onde se concentram deformações intensas (com ausência de deformações em outras regiões), e essas faixas seguem o tamanho da malha, isto é, assumem a largura da malha, buscando a faixa menor possível, conforme previsto pela teoria clássica do contínuo. Isto acarreta problemas bastante indesejáveis, deste a variação dos resultados de acordo com o tamanho da malha, até a situação, carente de sentido físico, de ruptura sem dissipação de energia.

\subsection{Plasticidade com Gradiente}

A localização de deformações tem sido um tema importante de análise, na tentativa de melhorar a simulação numérica do colapso de estruturas. Conforme visto na seção anterior, a presença de amolecimento por deformação nas equações constitutivas traz dificuldades fundamentais no âmbito das teorias do contínuo clássicas (ou locais). (BENALLAL et al. [5], [6], BENALLAL e TVERGAARD ${ }^{[7],}$ PIJAUDIER-CABOT e BENALLAL ${ }^{[45],}$ RICE $^{[48],}$ RUDNICK e RICE ${ }^{[50]}$.) Na seção anterior também ilustrou-se como materiais com amolecimento por deformação tornam-se matematicamente mal posicionados após o início da localização (perda de elipticidade das equações), porque o contínuo local (ou clássico) permite a formação de uma faixa infinitamente pequena na qual as deformações se concentram. As dificuldades numéricas que surgem em decorrência disso também foram analizadas. (ARMERO [2], MANZOLI ${ }^{[42]}$, BAZANT et al. ${ }^{[3], ~[4], ~ d e ~ B O R S T ~(1988) ~}{ }^{[10]}$ Várias abordagens tem sido propostas para superar essas dificuldades. Uma idéia é enriquecer a teoria do contínuo com relações constitutivas não convencionais de tal maneira que um comprimento interno ou característico seja introduzido. Teorias não locais desse tipo são o contínuo de Cosserat ERINGEN e EDELEN [23], de 
BORST (1991) ${ }^{\text {[11], FLECK e HUTCHINSON }}{ }^{[24]}$, as teorias dos gradientes de ordem mais elevada TRIANTAFYLLIDIS e AINFANTIS ${ }^{[57]}$, a teoria integral PIJAUDIERCABOT e BENALLAL ${ }^{[45]}$, e a teoria do gradiente LASRY e BELYTCHSKO ${ }^{\text {[37] }}$ MULHAUS e AINFANTIS ${ }^{[41]}$, de BORST e MULHAUS ${ }^{[12]}$.

Neste trabalho é utilizado o modelo de plasticidade com gradiente BENALLAL e TVERGAARD ${ }^{[7]}$ para pequenas deformações. Esse modelo consiste basicamente naquele descrito na seção sobre plasticidade, apenas modificando o critério de plastificação ( 3.8 ), fazendo o tamanho da superfície plástica $R$ depender agora não somente de $p$, mas também de seus sucessivos gradiente, mas tomando, por simplicidade, apenas o segundo gradiente, o laplaciano de $p, \nabla^{2} p$ :

$$
f\left(\sigma, p, \nabla^{2} p\right)=0
$$

Uma consequência dessa alteração é que uma relação explícita para o multiplicador plástico $\lambda^{\cdot}$, a partir da condição de consistência ( 3.13 ), como foi feito antes. Em vez disso, a relação de consistência ( $\mathbf{3 . 1 3}$ ) fornece uma equação diferencial parcial:

$$
\frac{\partial f}{\partial \sigma} \mathbf{C} \dot{\varepsilon}-H \dot{\lambda}+\omega \nabla^{2} \dot{\lambda}=0
$$

com

$$
H=h+\frac{\partial f}{\partial \sigma} C \frac{\partial F}{\partial \sigma},
$$




$$
\omega=\frac{\partial f}{\partial R} \frac{\partial R}{\partial\left(\nabla^{2} p\right)} \frac{\partial F}{\partial R}
$$

Outros termos aparecem em ( 3.30 ), mas eles podem ser omitidos aqui, pois dependem de $\partial F / \partial R$, que geralmente é uma constante e suas derivadas se anulam.

Analisando-se a expressão ( 3.30 ) conclui-se que $\omega$ tem a dimensão de $H$ multiplicado por comprimento ao quadrado:

$$
\omega=\alpha l^{2}
$$

onde $l$ é o comprimento característico e $\alpha$ é um parâmetro do material. ( $\omega$ vai controlar a largura da faixa de localização de deformações.)

O problema de valor de contorno continua sendo definido por ( 3.7 ) e ( 3.10 ), mas agora com ( 3.29 ) no lugar de ( 3.8 ), e com ( 3.12 ) (e ( 3.13 )) resultando na equação ( 3.30 ), o que significa que a equação para $\dot{\lambda}$ fica acoplada ao problema, e tem de ser resolvida no domínio plástico. 


\section{Equações Integrais}

\subsection{Equações integrais}

\subsubsection{Elasticidade}

A fim de obter as equações integrais utilizadas no método dos elementos de contorno, pode-se aplicar a segunda identidade de Green ( 2.32 ) ao operador diferencial da elasticidade ( 3.6 ) (equações de Navier). A identidade de Green é aplicada a dois campos de deslocamento correspondendo a estados elásticos distintos, expressando neste caso o teorema de Betti, como segue:

$$
\begin{gathered}
B\left(\mathbf{u}_{i}^{*}, \mathbf{u}\right)=\int_{\Omega}\left(-\mathrm{L}_{k l} u_{i l}^{*}\right) u_{k} d \Omega+\int_{\Gamma}\left(p_{i k}^{*} u_{k}-u_{i k}^{*} p_{k}\right) d \Gamma-\int_{\Omega} u_{i k}^{*}\left(-\mathrm{L}_{k l} u_{l}\right) d \Omega=0 \\
\text { ou } \\
B\left(\mathbf{u}_{i}^{*}, \mathbf{u}\right)=\int_{\Omega}\left(-\mathrm{L} \mathbf{u}_{i}^{*}\right) \mathbf{u} d \Omega+\int_{\Gamma}\left(\mathbf{p}_{i}^{*} \mathbf{u}-\mathbf{u}_{i}^{*} \mathbf{p}\right) d \Gamma-\int_{\Omega} \mathbf{u}_{i}^{*}(-\mathrm{L} \mathbf{u}) d \Omega=0,
\end{gathered}
$$

onde o estado elástico atual num corpo de domínio $\Omega$ e contorno $\Gamma$ é representado pelo campo de deslocamentos $\mathbf{u}$, com o vetor de tensão (forças de superfície) $\mathbf{p}$ e forças volumétricas $\mathbf{b} ; \mathbf{u}_{i}^{*}$ e $\mathbf{p}_{i}^{*}$ representam deslocamento e vetor de tensão devidos a uma carga concentrada na direção $i$ em um ponto de um meio infinito (solução fundamental de Kelvin).

As equações de Navier são reescritas abaixo: 


$$
\begin{gathered}
\left(-\mathrm{L}_{k l} u_{l}\right)=-G u_{k, l l}-\frac{G}{1-2 v} u_{l, l k}=b_{k} \\
\text { ou } \\
\mathrm{L}(\mathbf{u})=G \operatorname{div}(\operatorname{grad}(\mathbf{u}))^{T}+\frac{G}{1-2 v} \operatorname{grad} \operatorname{div}(\mathbf{u})=\mathbf{b},
\end{gathered}
$$

onde $G$ é o módulo de deformação transversal e $v$ é o coeficiente de Poisson.

Para o estado plano de tensão (que constitue o caso ds exemplos apresentados neste trabalho), as soluções fundamentais $\mathbf{u}_{i}^{*}$ e $\mathbf{p}_{i}^{*}$ são:

$$
\begin{gathered}
u_{i j}^{*}(s, q)=-\frac{1}{8 \pi(1-\bar{v}) G}\left[(3-4 \bar{v}) \ln r \delta_{i j}-r_{, i} r_{, j}\right] \\
\mathrm{e} \\
p_{i j}^{*}(s, q)=-\frac{1}{4 \pi(1-\bar{v}) r}\left\{\left[(1-2 \bar{v}) \delta_{i j}+r_{, i} r_{j}\right] \frac{\partial r}{\partial n}-(1-2 \bar{v})\left(r_{, i} n_{j}-r_{, j} n_{i}\right)\right\},
\end{gathered}
$$

onde $\bar{v}=v /(1+v)$, constituindo a correção para o estado plano de tensão (a expressões ( 4.3 ) valem também para o estado plano de deformação, substituindo-se $\bar{v}$ por $v)$. O primeiro índice em $u^{*}{ }_{i j} e p^{*}{ }_{i j}$ refere-se à direção de aplicação da carga no ponto fonte, e o segundo à direção do campo no ponto de observado.

Como o ponto fonte $s$ contém a singularidade, o teorema de Betti não é válido em $s$, e devendo-se tomar o domínio excluindo-se uma vizinhança em torno de $s$ e avaliando-se o limite: 


$$
\lim _{\varepsilon \rightarrow 0}\left\{\begin{array}{c}
\int_{\Omega-\Omega_{\varepsilon}}\left(-\mathrm{L}_{k l} u_{i l}^{*}\right) u_{k} d \Omega+\int_{\Gamma+\Gamma_{\varepsilon}}\left(p_{i k}^{*} u_{k}-u_{i k}^{*} p_{k}\right) d \Gamma \\
-\int_{\Omega-\Omega_{\varepsilon}} u_{i k}^{*}\left(-\mathrm{L}_{k l} u_{l}\right) d \Omega
\end{array}\right\}=0,
$$

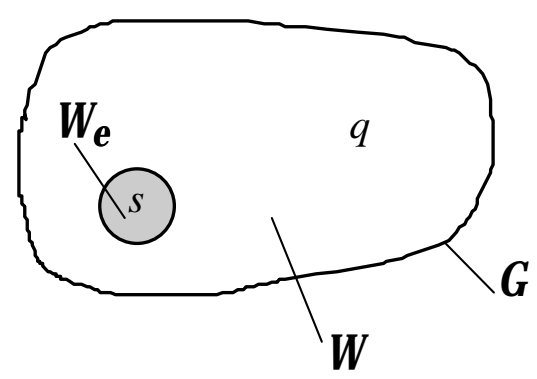

Figura 4.1 - Domínio com exclusão de região contendo o ponto singular.

A solução fundamental possui a seguinte propriedade:

$$
-L \mathbf{u}_{i}^{*}(s, q)=\mathbf{b}_{i}^{*}=\left\{\begin{array}{ll}
\infty & \text { se } s=q \\
0 & \text { se } s \neq q
\end{array} \quad(\text { delta de Dirac) }\right.
$$

onde $s$ representa o ponto de aplicação da carga na solução fundamental, e $q$ é o ponto de integração. Portanto, o primeiro termo da identidade de Green resulta nulo:

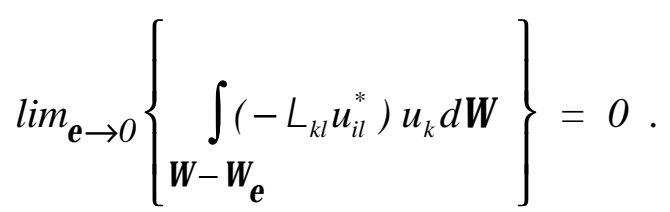

O campo de deslocamentos $u^{*}(s, q)$ possui singularidade de ordem menor, $O\left(r^{-}\right.$ $\left.{ }^{1}\right)$. Como $d \Omega=O\left(r^{-2}\right)$, mostra-se que: 


$$
\begin{gathered}
\lim _{\varepsilon \rightarrow 0}\left\{\int_{\Gamma_{\varepsilon}} u_{i k}^{*} p_{k} d \Gamma\right\}=0, \quad \lim _{\varepsilon \rightarrow 0}\left\{\int u_{i k}^{*}\left(-\mathrm{L}_{k l} u_{l}\right) d \Omega\right\}=0, \\
\lim _{\varepsilon \rightarrow 0}\left\{\int_{\Omega} u_{i k}^{*}\left(-\mathrm{L}_{k l} u_{l}\right) d \Omega\right\}=\int_{\Omega} u_{i k}^{*}\left(-\mathrm{L}_{k l} u_{l}\right) d \Omega .
\end{gathered}
$$

Quanto à integral em $p^{*}$, seu maior grau de singularidade faz surgir um termo livre:

$$
\begin{gathered}
\lim _{\varepsilon \rightarrow 0}\left\{\int_{\Gamma_{\varepsilon}} p_{i k}^{*}(s, q) u_{k}(q) d \Gamma_{\varepsilon}(q)\right\}=u_{k}(s) \int_{\Gamma_{\varepsilon}} p_{i k}^{*}(s, q) d \Gamma_{\varepsilon}(q)=c_{i k}(s) u_{k}(s) \\
\text { ou } \\
c_{i k}(s)=\int_{\Gamma_{\mathcal{E}}} p_{i k}^{*}(s, q) d \Gamma_{\varepsilon}(q),
\end{gathered}
$$

resultando

$$
c_{i k}(s) u_{k}(s)=\left\{\begin{array}{lll}
\delta_{i k} u_{k} & \text { se } & s \in \Omega \quad \text { (pontos do interior) } \\
\bar{c}_{i k} u_{k} & \text { se } & s \in \Gamma \\
0 & \text { se } & s \notin \Omega
\end{array}\right.
$$


e observando-se que o termo $\bar{c}_{i k}$ depende da geometria da superfície do corpo no ponto $s$, e vale 0.5 se o contorno é suave em torno do ponto $s$.

O teorema de Betti resulta então na indentidade de Somigliana:

$$
\begin{gathered}
c_{i k}(s) u_{k}(s)+\int_{\Gamma}\left(p_{i k}^{*} u_{k}-u_{i k}^{*} p_{k}\right) d \Gamma-\int_{\Omega} u_{i k}^{*} b_{k} \Omega=0 \\
\text { оu } \\
c_{i k}(s) u_{k}(s)+\int_{\Gamma}\left(\mathbf{p}_{i}^{*} \mathbf{u}-\mathbf{u}_{i}^{*} \mathbf{p}\right) d \Gamma-\int_{\Omega} \mathbf{u}_{i}^{*} \mathbf{b} d \Omega=0,
\end{gathered}
$$

A representação integral do campo de tensão pode ser obtida por diferenciação da identidade de Somigliana para deslocamentos, utilizando-se o tensor de deformação expresso através do gradiente de u na lei de Hooke, resultando em

$$
\sigma_{i j}=\int_{\Gamma} D_{i j k} p_{k} d \Gamma-\int_{\Gamma} S_{i j k} u_{k} d \Gamma+\int_{\Omega} D_{i j k} b_{k} d \Omega
$$

com os núcleos das integrais, no caso de estado plano de tensão, dados por

$$
\begin{gathered}
D_{i j k}=\frac{1}{4 \pi(1-\bar{v}) r}\left\{\left[(1-2 \bar{v})\left(r_{, j} \delta_{i k}+r_{, i} \delta_{j k}+r_{, k} \delta_{i j}\right)+2 r_{, i} r_{, j} r_{, k}\right\},\right. \\
S_{i j k}=\frac{G}{2 \pi(1-\bar{v}) r^{2}}\left\{2 \frac { \partial r } { \partial n } \left[(1-2 \bar{v})\left(r_{, k} \delta_{i j}+\bar{v}\left(r_{, j} \delta_{i k}+r_{, i} \delta_{j k}\right)-4 r_{, i} r_{, j} r_{, k}\right]+\right.\right. \\
\left.2 \bar{v}\left(n_{i} r_{, j} r_{, k}+n_{j} r_{, i} r_{, k}\right)+(1-2 \bar{v})\left(2 n_{k} r_{, i} r_{, j}+n_{j} \delta_{i k}+n_{i} \delta_{j k}\right)-(1-4 \bar{v}) n_{k} \delta_{i j}\right\},
\end{gathered}
$$

$\operatorname{com} n$ referindo-se ao vetor normal no contorno.

Note-se que as derivadas são em relação ao ponto fonte. Observe-se também que se o ponto fonte pertencer ao contorno, $S_{i j k}$ possui uma singularidade um grau acima da de $\mathbf{p}_{i}^{*}$, e precisa receber um cuidado especial na integração. (O termo com 
as forças volumétricas não apresenta grandes dificuldades, por apresentar menor singularidade, e não dá origem a termo livre, o mesmo ocorrendo com a integral de contorno em $\mathbf{u}_{i}^{*}$.) Na discretização pelo método dos elementos de contorno essa integral é frequentemente avaliada de modo numérico no contorno (diferenciação das funções de forma), a partir dos valores obtidos para os deslocamentos. A avaliação exata dessa integral está em discussão na literatura, tendo sido propostas várias alternativas. Este ponto voltará a ser tratado mais à frente.

\subsubsection{Problemas não lineares}

Em problemas não lineares o teorema de Betti (segunda identidade de Green) não pode ser aplicado diretamente. Antes de aplicá-lo, é necessário definir adequadamente o problema não linear, o que pode ser feito recorrendo-se à formulação de tensão e deformação iniciais, da teoria linear. Neste caso, o problema, que também deve ser dado em taxas, passa ser regido pelas equações abaixo, no lugar de ( 3.4 ):

$$
\begin{gathered}
\dot{\varepsilon}+\dot{\varepsilon}^{0}=\frac{1}{2}\left[(\nabla \otimes \dot{\mathbf{u}})^{T}+\nabla \otimes \dot{\mathbf{u}}\right] \\
\nabla \dot{\sigma}+\dot{\mathbf{b}}=\mathbf{0} \\
\dot{\sigma}+\dot{\sigma}^{0}=\mathbf{C} \dot{\varepsilon},
\end{gathered}
$$

que constituem um problema não linear porque a evolução dos campos de deformação e tensão iniciais $\left(\dot{\boldsymbol{\varepsilon}}^{\mathbf{0}}, \dot{\boldsymbol{\sigma}}^{\mathbf{0}}\right)$ depende não só do estado do material, como também das quantidades $(\dot{\varepsilon}, \dot{\sigma})$.

No algorítmo de tensões iniciais, são acrescentados incrementos apenas de $\boldsymbol{\sigma}^{\mathbf{0}}$, ou seja, $\varepsilon^{\mathbf{0}}=\mathbf{0}$, de modo que substituindo-se as equações anteriores umas nas outras encontra-se o seguinte forma para o operador de Navier: 


$$
\begin{gathered}
(-\mathrm{L} \dot{\mathbf{u}})=-\nabla(\nabla \otimes \dot{\mathbf{u}})-\frac{G}{1-2 v} \nabla(\nabla \dot{\mathbf{u}})=\dot{\mathbf{b}}-\nabla \dot{\sigma}^{0} \\
o u \\
\left(-\mathrm{L}_{k l} \dot{u}_{l}\right)=-G \dot{u}_{k, l l}-\frac{G}{1-2 v} \dot{u}_{l, l k}=\dot{b}_{k}-\dot{\sigma}_{k l, l}^{0} .
\end{gathered}
$$

Agora é possível aplicar a segunda identidade de Green, como na seção anterior, chegando-se à seguinte expressão, correspondente a (4.10):

$$
c_{i k} \dot{u}_{k}=\int_{\Gamma}\left[u_{i k}^{*}\left(\dot{p}_{k}-\dot{p}_{k}^{p}\right)-p_{i k}^{*} \dot{u}_{k}\right] d \Gamma+\int_{\Omega} u_{i k}^{*}\left(\dot{b}_{k}-\dot{\sigma}_{k l, l}^{p}\right) d \Omega .
$$

$\operatorname{com} \sigma^{\mathbf{p}}$ significando a especificação da não linearidade para o caso da plasticidade.

Utilizando-se a integração por partes no último termo da segunda integral na expressão anterior, tem-se

$$
\int_{\Omega} u_{i k}^{*}\left(-\dot{\sigma}_{k l, l}^{p}\right) d \Omega=-\int_{\Gamma} u_{i k}^{*} \dot{p}_{k}^{p} d \Gamma+\int_{\Omega} \varepsilon_{i k l}^{*} \dot{\sigma}_{k l}^{p} d \Omega
$$

o que permite escrever a identidade de Somigliana para a plasticidade utilizando tensão inicial:

$$
c_{i k} \dot{u}_{k}=\int_{\Gamma} u_{i k}^{*} \dot{p}_{k} d \Gamma-\int_{\Gamma} p_{i k}^{*} \dot{u}_{k} d \Gamma+\int_{\Omega} u_{i k}^{*} \dot{b}_{k} d \Omega+\int_{\Omega} \varepsilon_{i j k}^{*} \dot{\sigma}_{j k}^{p} d \Omega .
$$

Um procedimento análogo conduz à formulação para o algorítmo de deformações iniciais (impondo-se $\sigma^{0}=0$ ), chegando-se a uma expressão similar.

Da mesma forma que no caso elástico, a representação integral das tensões pode ser obtida por derivação da identidade de Somigliana e aplicação da lei de Hooke. Observe-se porém que a integral com o termo plástico requer também um 
cuidado especial na integração. Como a integral é de domínio, é possível reduzir o grau de singularidade que aparece com a derivação do núcleo (o que não é possível com a integral de contorno em $\mathbf{p}_{i}^{*}$, o caso mais crítico: $1 / r^{2}$ no caso plano).

Para o tratamento dessa integral, adotou-se a solução proposta por Telles ${ }^{[54]}$. A integração resulta num coeficiente de termo livre:

$$
g_{i j}\left(\dot{\sigma}_{m k}^{p}\right)=-\frac{1}{8}\left(2(1+\bar{v}) \dot{\sigma}_{i j}^{p}+(1-3 \bar{v}) \dot{\sigma}_{l l}^{p} \delta_{i j}\right)
$$

tomando-se o cuidado, nos casos planos, de considerar o trabalho na terceira dimensão, isto é, $\sigma_{33}$ no estado plano de deformação ou $\varepsilon_{33}$ no de tensão.

A representação integral das tensões (em pontos do interior) fica assim:

$$
\dot{\sigma}_{i j}=\int_{\Gamma} D_{i j k} \dot{p}_{k} d \Gamma-\int_{\Gamma} S_{i j k} \dot{u}_{k} d \Gamma+\int_{\Omega} D_{i j k} \dot{b} d \Omega+\int_{\Omega} \bar{E}_{i j m k} \dot{\sigma}_{m k}^{p} d \Omega+g_{i j}\left(\dot{\sigma}_{m k}^{p}\right),
$$

onde o termo $\bar{E}_{i j k l}$ surgiu da diferenciação da integral plástica e é dado por

$$
\begin{aligned}
& \bar{E}_{i j k l}=\frac{1}{4 \pi(1-\bar{v}) r^{2}}\left\{(1-2 \bar{v})\left[\delta_{i k} \delta_{l j}+\delta_{j k} \delta_{l i}-\delta_{i j} \delta_{k l}+2 \delta_{i j} r_{, k} r_{, l}\right)+\right. \\
& \left.2 \bar{v}\left[\delta_{l i} r_{, j} r_{, k}+\delta_{j k} r_{, l} r_{i}+\delta_{i k} r_{l,} r_{, j}+\delta_{j l} r_{, i} r_{, k}\right]+2 \delta_{k l} r_{i} r_{, j}-8 r_{, i} r_{, j} r_{, k} r_{, l}\right\} .
\end{aligned}
$$

\subsubsection{Representação integral de tensão no contorno}

A representação integral de tensão, equação ( 4.19 ), foi derivada apenas para pontos internos. A fim de estender aquela equação para pontos do contorno, é preciso encontrar o limite quando um ponto interno $s$ se aproxima do contorno. Desse modo é possível encontrar a representação integral no contorno. Alternativamente, as 
tensões no contorno podem ser calculadas diretamente dos valores já obtidos no contorno - um procedimento usado com muita freqüência. As componentes normal e tangencial podem ser aproximadas a partir dos valores das reações já computadas. A componente normal na direção do contorno pode ser obtida numericamente utilizando-se os valores dos deslocamentos já computados, derivando-se as funções de forma. Essa abordagem é largamente utilizada, garantindo bons resultados, dentro de um certo limite de precisão. No entanto, a equação integral é a representação exata para a determinação da tensão. Como no presente caso a intenção era obter os resultados os mais precisos possíveis, o que parecia importante neste contexto, preferiu-se obter a representação exata.

A significativa melhoria na resposta obtida com a utilização da representação integral da tensão no contorno, em comparação com o método tradicional (derivação das funções de forma) já havia sido demonstrada para o caso da elasticidade, por exemplo, por GUIGGIANI ${ }^{[33]}$. Em artigo bastante recente, POON et al. ${ }^{[46]}$ constatam certas perturbações na resposta em exemplos da plasticidade tratados com o método tradicional, as quais eles acreditam estar relacionadas com a forma de cálculo da tensão no contorno. Essas perturbações, em forma de oscilações, são amenizadas por aqueles autores recorrendo a uma correção da curva de resposta através de uma curva média, pós-processamento. Eles sugerem a utilização de um procedimento como o utilizado Guiggiani para evitar essas perturbações e obter um melhoria geral da resposta, embora não tenham ainda realizado essa tentativa. No caso presente, onde está envolvido o problema da localização, é possível que a utilização do método tradicional acarrete conseqüências ainda mais negativas, talvez dificultando a captura da localização de deformações. Como os exemplos mostrados neste trabalho utilizam todos a formulação com a representação integral da tensão no contorno, não foi feita uma avaliação desse ponto. Mas seria interessante comparar uma versão exatamente igual do programa computacional aqui utilizado, exceto quanto à utilização do método tradicional de cálculo da tensão no contorno, para verificar essa questão. 
A obtenção da representação integral exata para as tensões não é tão simples, devido à presença de integrais fortemente singulares, como as que exibem núcleos de singularidade $1 / r^{2}$ e $1 / r^{3}$ para problemas $2 \mathrm{D}$ e $3 \mathrm{D}$, respectivamente. Diversas contribuições tem sido feitas a este tema ainda aberto na literatura CRUSE e

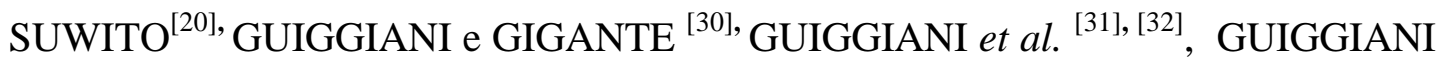

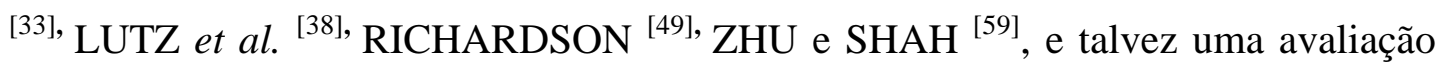
mais consistente dessas alternativas já comece a ser vislumbrada (uma boa exposição em RICHARDSON ${ }^{[49]}$ ). Neste trabalho, adotou-se a abordagem proposta em GUIGGIANI et al. ${ }^{[31]}$ GUIGGIANI ${ }^{[33]}$. Uma diferença, para a extensão dessa abordagem à plasticidade, é que foi necessário adicionar o termo com a integral de domínio, por ser preciso levar em consideração o tensor plástico.

A fim de se obter uma expressão similar à equação ( 4.19 ), válida também para pontos no contorno, deve-se atentar para as novas singularidades em questão. Conforme observado anteriormente, é preciso realizar uma diferenciação e aplicar a lei de Hooke. A diferenciação é realizada com relação ao ponto fonte, agora situado no contorno. Portanto, uma expressão distinta de ( 4.19 ) será obtida, resultando um novo termo livre devido ao caráter hipersingular do núcleo da integral de contorno.

A diferenciação deve ser feita antes de se transformar a segunda identidade de Green (ou teorema de Betti) na identidade de Somigliana. Embora em seus artigos Guiggiani use a diferenciação do teorema de Betti, aparentemente nada impede que se aplique o tensor de rigidez às derivadas daquele teorema, arranjadas de acordo com a lei de Hooke. Então, fazendo-se a diferenciação e aplicando-se a lei de Hooke (a partir da identidade de Green), obtém-se

$$
\lim _{\varepsilon \rightarrow 0}\left\{\int_{\left(\Gamma-e_{\varepsilon}\right)+\Gamma_{\varepsilon}} D_{i j k} \dot{p}_{k} d \Gamma-\int_{\left(\Gamma-e_{\varepsilon}\right)+\Gamma_{\varepsilon}} S_{i j k} \dot{u}_{k} d \Gamma+\int_{\Omega-\Omega_{\varepsilon}} E_{i j k l} \dot{\sigma}_{k l} d \Omega\right\}=0 .
$$

O termo de volume não acarreta em termo livre, e não é considerado aqui, por simplicidade.

O limite acima pode ser resolvido removendo-se uma vizinhança do ponto singular, o ponto fonte $S$, definido por $\Gamma_{\varepsilon}, \Omega_{\varepsilon}$ e $\rho_{\varepsilon}$, como mostrado na Figura 4.2. 


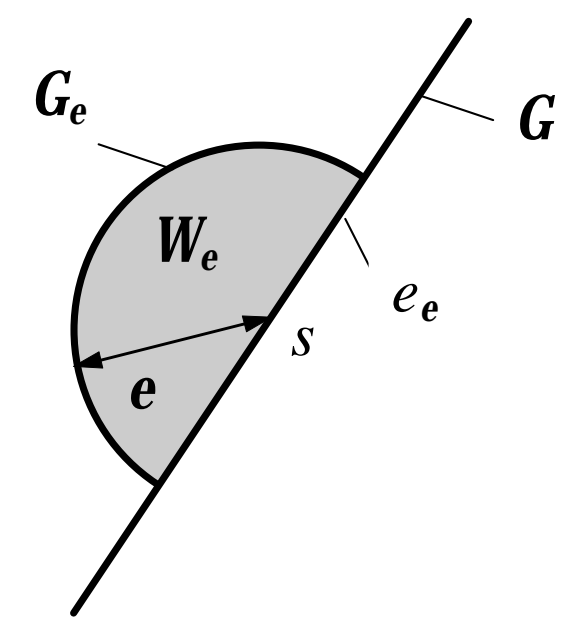

Figura 4.2 - Região infinitesimal em torno do ponto fonte $S$.

A estratégia consiste em assumir que o campo de deslocamentos e sua primeira derivada satisfazem a condição de Hölder $\left(u_{k} \in C^{l} \mathrm{em} s\right)$. A restriçãa à classe $C^{l}$ não é necessária para a existência do limite ( 4.21 ), sendo imposta apenas porque permite fornecer um procedimento suficiente para a regularização das integrais, eliminando as singularidades. Adotando-se esta restrição é possível escrever a seguinte expansão (no caso 2D):

$$
\begin{aligned}
& \dot{u}_{k}(q)=\dot{u}_{k}(s)+\dot{u}_{k, m}(s)\left(x_{m}(q)-x_{m}(s)\right)+O\left(r^{2}\right) \\
& \dot{p}_{k}(q)=\dot{\sigma}_{k m}(q) n_{m}(q)=\dot{\sigma}_{k m}(s) n_{m}(q)+O(r),
\end{aligned}
$$

que substituída na expressão anterior resulta em 


$$
\begin{aligned}
& \lim _{\varepsilon \rightarrow 0}\left\{\int_{\left(\Gamma-e_{\varepsilon}\right)}\left[D_{i j k} \dot{p}_{k}(q)-S_{i j k} \dot{u}_{k}(q)\right] d \Gamma_{\left(\Gamma-e_{\varepsilon}\right)}\right\} \\
& +\lim _{\varepsilon \rightarrow 0}\left\{\begin{array}{c}
\int_{\Gamma_{\varepsilon}}\left\{D_{i j k}\left(\dot{p}_{k}(q)-\dot{\sigma}_{k m}(s) n_{m}(q)\right)\right. \\
\left.-S_{i j k}\left[\dot{u}_{k}(q)-\dot{u}_{k}(s)-\dot{u}_{k, m}(q)\left(x_{m}(q)-x_{m}(s)\right)\right]\right\} d \Gamma_{\varepsilon}
\end{array}\right\} \\
& +\lim _{\varepsilon \rightarrow 0}\left\{\int_{\Gamma_{\varepsilon}}\left\{D_{i j k} \dot{\sigma}_{k m}(s) n_{m}(q)-S_{i j k}\left[\dot{u}_{k}(s)+\dot{u}_{k, m}(s)\left(x_{m}(q)-x_{m}(s)\right)\right]\right\} d \Gamma_{\varepsilon}\right\} \\
& +\lim _{\varepsilon \rightarrow 0}\left\{\int_{\Omega-\Omega_{\varepsilon}} \bar{E}_{i j k l} \dot{\sigma}_{k l} d \Omega_{\Omega-\Omega_{\varepsilon}}\right\}=0,
\end{aligned}
$$

onde, em 2D, ${ }_{i j k l} \dot{k}_{k l}=0.5^{\cdot}{ }_{i j}$ para pontos de contorno suave (admitidos neste trabalho como retos).

É conveniente reescrever a expressão anterior como

$$
I_{1}+I_{2}+I_{3}+I_{4}=0
$$

onde cada termo representa um dos limites expressos em ( 4.23 ).

O limite $I_{1}$ precisa ser avaliado com cuidado, como se verá abaixo.

O limite $I_{2}$ é igual a zero, de acordo com a expansão ( 4.22 ) e considerando-se que $d \Gamma_{\varepsilon}=O(\varepsilon)$ em $\Gamma_{\varepsilon}$ para o caso $2 \mathrm{D}$ (ou $d \Gamma_{\varepsilon}=O\left(\varepsilon^{2}\right)$ em 3D).

Quanto ao limite $I_{3}$, pode ser rearranjado da seguinte maneira:

$$
\begin{gathered}
\left.\left.\lim \left\{\int_{\Gamma_{\varepsilon}} D \dot{\sigma}(s) \quad q\right)-S \cdot\left(\begin{array}{lll}
s & x()-x & s
\end{array}\right)\right\} d \Gamma_{\varepsilon}\right\} \\
-\quad \varepsilon\left\{(s) \int_{\Gamma_{\varepsilon}} S d \Gamma\right\},
\end{gathered}
$$

de modo que o primeiro e o segundo limites da expressão acima resultam respectivamente no primeiro e segundo termos seguintes: 


$$
{ }_{3}=c_{i j k l}(s) \dot{\sigma}_{k l}(\quad)-\lim _{\varepsilon \rightarrow 0}\left\{\dot{u}_{k}(s) \frac{i j k}{\varepsilon}\right\}
$$

onde $c_{i j k l}$ e $d_{i j k}$ são termos livres que dependem da geometria na vizinhança do ponto S no contorno.

$c_{i j k l}$ é similar ao termo $c_{i j}$ que aparece na expressão ( 4.17 ). Para pontos de contorno suave, $c_{i j k} \dot{\sigma}_{k l}=0.5 \dot{\sigma}_{i j}$.

$d_{i j k}$ é o novo termo livre, que precisa ser avaliado de maneira apropriada. Ele resulta num valor infinito quando $\varepsilon \rightarrow 0$, mas é cancelado com um dos termos que resultam da integral $I_{l}$, proveniente do núcleo $S_{i j k}$. Ou seja, as expressões que precisam ser analisadas com maior cuidado provem das integrais $I_{1}$ e $I_{3}$. Mais especificamente, a maior dificuldade é encontrada nos seguintes limites, contidos naquelas integrais, $I_{1}$ e $I_{3}$ :

$$
\lim _{\varepsilon \rightarrow 0}\left\{\int_{\left(\Gamma-e_{\varepsilon}\right)} S_{i j k}(s, q) \dot{u}_{k}(q) d \Gamma_{\left(\Gamma-e_{\varepsilon}\right)}+\dot{u}_{k}(q) \frac{d_{i j k}(s)}{\varepsilon}\right\} .
$$

Os últimos passos na regularização das integrais, eliminando-se as singularidades, só serão dados após a discretização. A maneira precisa como isso ocorre depende da discretização. Mas o importante a ressaltar é que, seja qual for a discretização adotada, a presente formulação garantirá sempre o cancelamento das singularidades, desde que os termos singulares resultantes de $I_{1}$ e $I_{3}$ sejam considerados em conjunto, possibilitando seu cancelamento recíproco. Observe-se também que o termo de $I_{l}$ cujo núcleo é $D_{i j k}$ possui singularidade mais suave, mas pode ser tratado de forma análoga ao que é descrito a seguir, com respeito aos termos singulares da expressão anterior.

A regularização deve ser obtida no contexto da discretização. Conforme procedimento habitual, em cada elemento de contorno o campo de deslocamentos 
pode ser descrito em termos de funções de forma e pontos nodais. Num ponto nodal $\mathbf{x}:$

$$
u_{i}(\mathbf{x})=M^{c}(\xi(\mathbf{x})) u_{i}^{c}
$$

onde $\xi$ representa coordenadas intrínsecas e $M^{c}$ representa as funções de forma. No caso plano, $\xi$ é unidimensional, e $\xi \in[-1,+1]$. De maneira análoga, é possível o mapeamento da geometria de um elemento no contorno do espaço utilizado para o de coordenadas intrínsecas $\xi$ :

$$
x_{i}(\xi)=N^{c}(\xi) x_{i}^{c},
$$

onde novamente $N^{c}$ representa funções de forma. O comprimento de arco elementar também precisa ser expresso nas coordenadas intrínsecas:

$$
d \Gamma=J(\xi) d \xi_{1} \ldots d \xi_{n}
$$

onde $J(\xi)$ representa o jacobiano da transformação de coordenadas e $n$ a dimensão de $\xi$.

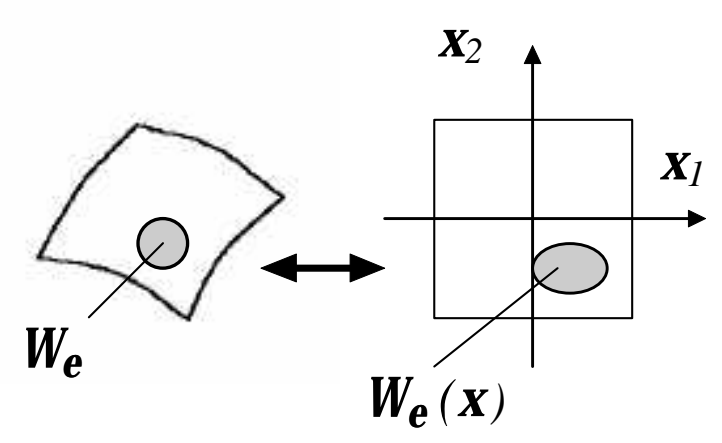

Figura 4.3 - Elemento de contorno em coordenadas intrínsecas 
A expressão ( 4.27 ) pode então ser expressa (em 3D) como

$$
I_{s}=\lim _{\varepsilon \rightarrow 0}\left\{\int_{\left(\Gamma-e_{\varepsilon}\right)_{\xi}} S_{i j k}(\eta, \xi) N^{c}(\xi) J(\xi) d \xi_{I} d \xi_{2}+N^{c}(\eta) \frac{d_{i j k}(s)}{\varepsilon}\right\},
$$

(4.31)

onde $\eta$ é a imagem do ponto fonte $\mathbf{s}$ em coordenadas intrínsecas.

Definindo-se agora um sistema polar de coordenadas com centro em $\eta$ (imagem de s), ou seja,

$$
\begin{gathered}
\xi_{l}=\eta_{1}+\rho \operatorname{sen} \theta \\
\xi_{2}=\eta_{2}+\rho \operatorname{sen} \theta \\
e \\
d \xi_{l} d \xi_{2}=\rho d \rho d \theta,
\end{gathered}
$$

a integral $I_{s}$ pode ser escrita como

$$
I_{s}=\lim _{\varepsilon \rightarrow 0}\left\{\int_{0}^{2 \pi} \int_{\alpha(\varepsilon, \theta)}^{\bar{\rho}(\theta)} Z_{i j k}(\rho, \theta) d \rho d \theta+N^{c}(\eta) \frac{d_{i j k}(s)}{\varepsilon}\right\},
$$

onde $\alpha(\varepsilon, \rho)$ representa a vizinhança de raio $\varepsilon$ nas coordenadas intrínsecas polares, $\bar{\rho}$ é a extremidade do elemento na direção $\theta$, e

$$
Z_{i j k}(\rho, \theta) d \rho d \theta=S_{i j k}(\eta, \xi) N^{c}(\xi) J(\xi) d \xi_{I} d \xi_{2}
$$

O integrando $Z$ da expressão acima pode ser expandido em série de Laurent: 


$$
Z_{i j k}(\rho, \theta)=\frac{Z_{(-2) i j k}(\theta)}{\rho^{2}}+\frac{Z_{(-1) i j k}(\theta)}{\rho}+O(1) .
$$

Esta expansão pode ser obtida de forma metódica (ver especialmente GUIGGIANI et al. ${ }^{[32]}$ e GUIGGIANI ${ }^{[33]}$ ). Para isso, é importante dispor da expansão em série de Taylor de $\alpha(\varepsilon, \rho)$, a vizinhança do ponto singular em coordenadas polares intrínsecas:

$$
\alpha(\varepsilon, \theta)=\varepsilon \beta(\theta)+\varepsilon^{2} \gamma(\theta)+O\left(\varepsilon^{3}\right) .
$$

Outras quantidades que aparecem na expressão de $Z$ também devem ser expandidas em séries de Taylor em torno do ponto singular.

Somando-se e subtraindo-se os termos singulares de $Z$, a integral $I_{s}$ torna-se

$$
I_{s}=\lim _{\varepsilon \rightarrow 0}\left\{\begin{array}{c}
\int_{0}^{2 \pi} \int_{\alpha(\varepsilon, \theta)}^{\bar{\rho}(\theta)}\left(Z_{i j k}(\rho, \theta)-\left(\frac{Z_{(-2) i j k}(\theta)}{\rho^{2}}+\frac{Z_{(-1) i j k}(\theta)}{\rho}\right)\right) d \rho d \theta \\
+\int_{0}^{2 \pi} \int_{\alpha(\varepsilon, \theta)}^{\bar{\rho}(\theta)} \frac{Z_{(-1) i j k}(\theta)}{\rho} d \rho d \theta \\
+\left(\int_{0}^{2 \pi} \int_{\alpha(\varepsilon, \theta)}^{\bar{\rho}(\theta)} \frac{Z_{(-2) i j k}(\theta)}{\rho^{2}} d \rho d \theta+N^{c}(\eta) \frac{d_{i j k}(s)}{\varepsilon}\right)
\end{array}\right\},
$$

O primeiro termo no limite acima é claramente regular, de acordo com a expansão de $Z$ na equação ( 4.35 ).

No segundo termo, após integração em $\rho$ e utilização da expansão de $\alpha(\varepsilon, \rho)$ em série de Taylor (truncada no primeiro termo), resulta 


$$
I_{s(-1)}=\lim _{\varepsilon \rightarrow 0}\left\{\int_{0}^{2 \pi} \int_{\alpha(\varepsilon, \theta)}^{\bar{\rho}(\theta)} \frac{Z_{(-1) i j k}(\theta)}{\rho} d \rho d \theta\right\}=\int_{0}^{2 \pi} Z_{(-1) i j k}(\theta) \ln \left|\frac{\bar{\rho}(\theta)}{\beta(\theta)}\right| d \theta
$$

tendo sido utilizada a seguinte propriedade intrínseca de $Z_{(-1)}$ :

$$
\int_{0}^{2 \pi} Z_{(-1) i j k}(\theta) d \theta=0
$$

que é conseqüência da própria solução fundamental.

$\mathrm{O}$ último termo em $Z$, que possui a maior singularidade, recebe tratamento análogo, utilizando-se as quantidades desenvolvidas em série de Taylor e integrandose em $\rho$, obtendo-se

$$
\begin{gathered}
I_{s(-2)}=\lim _{\varepsilon \rightarrow 0}\left\{\int_{0}^{2 \pi} \int_{\alpha(\varepsilon, \theta)}^{\bar{\rho}(\theta)} \frac{Z_{(-2) i j k}(\theta)}{\rho^{2}} d \rho d \theta+N^{c}(\eta) \frac{d_{i j k}(s)}{\varepsilon}\right\}= \\
=\lim _{\varepsilon \rightarrow 0}\left\{\int_{0}^{2 \pi} \frac{Z_{(-2) i j k}(\theta)}{\beta(\theta)} d \theta+N^{c}(\eta) \frac{d_{i j k}(s)}{\varepsilon}\right\} \\
-\int_{0}^{2 \pi} Z_{(-2) i j k}(\theta)\left(\frac{\gamma(\theta)}{\beta^{2}(\theta)}+\frac{1}{\bar{\rho}(\theta)}\right) d \theta \\
I_{s(-2)}=\int_{0}^{2 \pi} Z_{(-2) i j k}(\theta)\left(\frac{\gamma(\theta)}{\beta^{2}(\theta)}+\frac{1}{\bar{\rho}(\theta)}\right) d \theta
\end{gathered}
$$

pois 


$$
\int_{0}^{2 \pi} \frac{Z_{(-2) i j k}(\theta)}{\beta(\theta)} d \theta+N^{c}(\eta) \frac{d_{i j k}(s)}{\varepsilon}=0,
$$

( 4.41$)$

ou seja, os termos singulares cancelam-se mutuamente.

Reunindo-se esses resultados, $I_{s}$ resulta nos seguintes termos integrais, todos regulares:

$$
\begin{aligned}
& I_{s}=\int_{0}^{2 \pi} \int_{\alpha(\varepsilon, \theta)}^{\bar{\rho}(\theta)}\left(Z_{i j k}(\rho, \theta)-\left(\frac{Z_{(-2) i j k}(\theta)}{\rho^{2}}+\frac{Z_{(-1) i j k}(\theta)}{\rho}\right)\right) d \rho d \theta \\
& +\int_{0}^{2 \pi}\left(Z_{(-1) i j k}(\theta) \ln \left|\frac{\bar{\rho}(\theta)}{\beta(\theta)}\right|+Z_{(-2) i j k}(\theta)\left(\frac{\gamma(\theta)}{\beta^{2}(\theta)}+\frac{1}{\bar{\rho}(\theta)}\right)\right) d \theta .
\end{aligned}
$$

Ainda falta analisar o quarto termo, $I_{4}$, da expressão ( 4.23 ). Mas antes algumas observações acerca do procedimento descrito acima.

O que há a ressaltar neste procedimento, além da solução das singularidades, é sua generalidade, desde que sejam satisfeitas suas condições. É possível aplicar essa técnica a qualquer análise pelo método dos elementos de contorno, deixando aberta também a possibilidade de emprego de qualquer tipo de elementos ou grau de funções de forma. Uma desvantagem é a restrição de primeira derivada dos deslocamentos contínua nos pontos de colocação, para que a técnica possa ter efeito. Uma segunda desvantagem é a de que se torna necessário obter os termos regularizados em função das características da discretização, o que significa realizar todos os cálculos indicados acima para cada estrutura de discretização ou novo tipo de análise desejada.

Recentemente tem surgido uma estratégia alternativa que também parece bastante interessante (ver RICHARDSON ${ }^{[49]}$, como uma referência básica). Neste caso, a regularização por relaxamento é obtida considerando-se a identidade de Somigliana em conjunção a um estado de tensão composto de dois particulares estados: um de tensão constante e outro de tensão linear. A equação integral de 
tensão que resulta possui apenas singularidade fraca, evitando assim as dificuldades envolvidas na regularização de singularidades fortes habituais. Entre as vantagens dessa da regularização por relaxamento estão a não exigência de continuidade da derivada dos deslocamentos nos pontos singulares, e os procedimentos mais genéricos e robustos, no sentido de não ser necessário particularizar expressões de cálculo em função das características da aproximação. Uma desvantagem é que ao relaxar as singularidades alterando as equações integrais originais, aparentemente se perdem também parte das informações daquelas relações primitivas. Isto porque, na formulação com singularidades fracas assim obtida, a aproximação tende a exigir funções de forma de grau bem mais elevado. Nos primeiros resultados obtidos com esta técnica, constata-se que a precisão obtida por outros métodos necessita de funções de forma de grau duas vezes menor que o necessário pela formulação com singularidade fraca.

Essa área parece estar em plena pesquisa nos últimos anos, e progressos tem sido efetuados. Também parece haver indícios de que uma avaliação mais completa dessas estratégias se apresenta mais próxima.

Agora de volta à integral $I_{4}$ da expressão ( 4.23 ). Embora o método descrito acima tenha sido construído para a regularização das representações integrais das tensões no contorno, não parece muito difícil adaptá-lo para superar as singularidades no interior do domínio. Por outro lado, as integrais de tensão no interior do domínio possuem singularidade menor que a do contorno, e soluções para essas singularidades tem sido utilizadas muito antes. No presente trabalho o tratamento das integrais de tensão no domínio seguiram o procedimento utilizado em TELLES ${ }^{[54]} \mathrm{e}$ VENTURINI ${ }^{[58]}$. (Na verdade, o procedimento neste caso é similar ao de Guiggiani, embora particular e exato, enquanto no de Guiggiani é geral e sistemático, utilizando expansões em séries.)

$\mathrm{O}$ termo $I_{4}$ é importante porque dá origem a um termo livre, cujos valores para pontos no interior do domínio são conhecidos dos trabalhos de Telles e Venturini. Para pontos no contorno, é preciso calcular ainda os valores desse termo livre, que dependem da geometria em torno do ponto singular. 
A derivação do termo livre proposta neste trabalho parece mais direta que a proposta por TELLES ${ }^{[54]}$, que utiliza a fórmula de Leibniz para derivadas de integrais, também que a de HARTMANN ${ }^{[35]}$, embora talvez tenha mais similaridade com esta última.

Para obtenção do termo livre, é conveniente considerar-se o termo de domínio que possui a contribuição plástica do teorema de Betti, antes da aplicação do tensor de rigidez, ou seja,

$$
\begin{gathered}
\lim _{\varepsilon \rightarrow 0}\left\{\frac{\partial}{\partial x_{l}} \underset{\substack{\Omega-\Omega \\
\varepsilon}}{\varepsilon_{i j k}^{*} \dot{\sigma}_{j k}^{p} d \Omega}\right\}= \\
=\lim _{\varepsilon \rightarrow 0}\left\{\int_{\Omega} \frac{\partial \varepsilon_{i j k}^{*}}{\partial x_{l}} \dot{\sigma}_{j k}^{p} d \Omega-\int_{\varepsilon} \frac{\partial \varepsilon_{i j k}^{*}}{\partial x_{l}} \dot{\sigma}_{j k}^{p} d \Omega\right\},
\end{gathered}
$$

lembrando-se que a derivação é em relação ao ponto singular.

O primeiro termo no limite anterior é limitado. Isto pode ser mostrado, no caso plano por simplicidade, utilizando-se coordenadas polares $(\rho, \theta)$ em torno do ponto singular e considerando-se a propriedade da solução fundamental ser expressa nesse sistema como

$$
\varepsilon_{i j k}^{*}=\frac{\psi_{i j k}(\theta)}{r}
$$

onde $\psi_{i j k}(\theta)$ é uma função em $\theta$ somente. Como o objetivo é apenas determinar a existência do limite do primeiro termo no lado direito de ( 4.43 ), é interessante reescreve-lo como 


$$
\lim _{\varepsilon \rightarrow 0}\left\{\int_{\Omega} \frac{\partial \varepsilon_{i j k}^{*}}{\partial x_{l}} \dot{\sigma}_{j k}^{p} d \Omega\right\}=\lim _{\varepsilon \rightarrow 0}\left\{\frac{\partial}{\partial x_{l}} \int_{\theta_{1}}^{\theta_{2}^{2}} \int_{0}^{R(\theta)} \frac{\psi_{i j k}^{*}(\theta)}{r} \dot{\sigma}_{j k}^{p} r d r d \theta\right\}
$$

A singularidade $\left(O\left(r^{-1}\right)\right)$ no integrando é cancelada, e a expressão parece perfeitamente limitada. Falta então determinar o valor do termo livre, o que pode ser feito a partir do segundo termo em ( 4.43 ), com auxílio do teorema da divergência:

$$
\begin{gathered}
\int_{\varepsilon} \frac{\partial}{\partial x_{l}}\left(\varepsilon_{i j k}^{*} \dot{\sigma}_{j k}^{p}\right) d \Omega=\int_{\Gamma_{\varepsilon}}\left(\varepsilon_{i j k}^{*} \dot{\sigma}_{j k}^{p}\right) n_{l} d \Gamma_{\varepsilon}= \\
=\int_{\Gamma_{\varepsilon}} \varepsilon_{i j k}^{*}\left(\dot{\sigma}_{j k}^{p}-\dot{\sigma}_{j k}^{p}(s)\right) n_{l} d \Gamma_{\varepsilon}+\dot{\sigma}_{j k}^{p}(s) \int_{\Gamma_{\varepsilon}} \varepsilon_{i j k}^{*} n_{l} d \Gamma_{\varepsilon} .
\end{gathered}
$$

Considerando-se imposições sobre a continuidade da tensão (Hölder), a primeira integral se anulará quando avaliado o limite. Lembrando-se que $n_{l}(q)=-r_{, l}(s)$, o termo resultante fica 


$$
\begin{gathered}
\lim _{\varepsilon \rightarrow 0}\left\{\begin{array}{c}
-\dot{\sigma}_{j k}^{p}(s) \int_{i j k}^{*} r_{, l} d \Gamma_{\varepsilon} \\
\Gamma_{\varepsilon}
\end{array}\right\} \\
=\lim _{\varepsilon \rightarrow 0}\left\{\begin{array}{c}
\theta_{2} \varepsilon \\
-\dot{\sigma}_{j k}^{p}(s) \int_{\theta_{1}}^{\theta_{1}} \frac{\psi_{i j k}^{*}(\theta)}{r} r_{, l} r d \theta
\end{array}\right\}= \\
=-\dot{\sigma}_{j k}^{p}(s) \int_{2} \psi_{i j k}^{*} r_{l, l} d \theta . \\
\theta_{1}
\end{gathered}
$$

Observe-se que o termo livre depende da geometria. Normalmente ele é obtido para pontos do interior, $\left[\theta_{1}, \theta_{2}\right]=[0,2 \pi]$. Mas no presente caso ele deve ser avaliado em pontos do contorno. Adotou-se um contorno suave, ou seja, $\left[\theta_{1}, \theta_{2}\right]=[0, \pi]$. Calculando-se os termos e agrupando-se de acordo com a lei de Hooke, obtém-se o coeficiente do termo livre para pontos no contorno (suave):

$$
g_{i j}\left(\dot{\sigma}_{m k}^{p}\right)=-\frac{1}{16}\left(2(1+\bar{v}) \dot{\sigma}_{i j}^{p}+(1-3 \bar{v}) \dot{\sigma}_{l l}^{p} \delta_{i j}\right)
$$

justamente a metade do valor para pontos no interior.

\subsubsection{Plasticidade com gradiente}

Como visto no capítulo anterior, para resolver o problema da plasticidade com gradiente, é preciso levar em conta a equação do multiplicador plástico $\lambda$ (( 3.30 )). Neste caso, o valor escalar $\lambda$ não depende somente do estado local da tensão, 
como no caso do contínuo clássico (equação ( 3.14 )), sendo definido por uma equação diferencial que está assim acoplada às equações de equilíbrio na plasticidade.

Uma solução mais para esse problema das equações da plasticidade acopladas à do multiplicador plástico seria a solução simultânea dessas equações. ${ }^{[12],{ }^{[44]} \mathrm{Ou}}$ seja, no problema discretizado, seria resolvido um único sistema de equações algébricas, com o multiplicador plástico (definido na região plastificada) incluído entre as incógnitas.

Outra possibilidade ${ }^{[17],[18]}$ seria resolver o problema acoplado a partir de uma formulação modificada. (Maier et al. ${ }^{[39]}$ propõem esta abordagem para o método dos elementos de contorno, embora sem implementá-la.) Nesta segunda abordagem, o problema de valor de contorno da plasticidade com gradiente é substituído por uma aproximação variacionalmente consistente, em termos de variáveis generalizadas, na qual aparece apenas o primeiro gradiente do multiplicador plástico (em vez do segundo na formulação original), de modo que o sistema algébrico resultante é formalmente análogo ao obtido na plasticidade local (com variáveis generalizadas). Várias conseqüências decorrem, como a redução do grau das funções interpoladoras que devem ser utilizadas para a discretização do multiplicador plástico (de $C^{l}$ para $C^{0}$ ), pelo método dos elementos finitos.

Uma alternativa mais simples, fácil de implementar pelo método dos elementos de contorno, consiste em se resolver os dois problemas separadamente. Esta foi a estratégia adotada, o que parece razoável. Neste caso, a equação do multiplicador plástico é resolvida primeiramente, e seus valores utilizados nas outras equações, como na plasticidade local.

Para resolver a equação do multiplicador plástico através dos elementos de contorno, deve-se transformá-la primeiro numa representação integral, o que não é difícil, conforme pode-se observar na literatura (em Brebbia et al. ${ }^{[15]}$, por exemplo). Há diversas maneiras de se obter essa representação integral. Aqui será escolhida uma particular solução fundamental, utilizando-se o método da colocação. Seria possível tomar uma solução fundamental que considerasse o termo linear em $\lambda^{\cdot}$, o que permitiria evitar alguma indesejável integral de domínio. Mas devido à presença do campo de deformação, o primeiro termo da equação( 3.30 ) ainda permaneceria. 
Entretanto, a solução mais simples a escolher neste caso seria a solução para o problema de Laplace, dada por

$$
\lambda^{*}=\frac{1}{2 \pi} \ln (r)
$$

e seu fluxo,

$$
\frac{\partial \lambda^{*}}{\partial m}=-\frac{1}{2 \pi} r_{, i}
$$

onde $r$ é a distância entre o ponto fonte e o de integração em um domínio infinito bidimensional, e $m$ representa a derivada direcional na direção do vetor normal à interface elástico-plástica.

Como o operador escolhido foi o de Laplace, tem-se:

$$
-\left\llcorner\lambda=-\omega \nabla^{2} \dot{\lambda}=\frac{\partial f}{\sigma} \mathbf{C} \dot{\varepsilon}-H \dot{\lambda}\right.
$$

Aplicando-se a segunda identidade de Green, resulta

$$
c \dot{\lambda}=\int_{\Gamma} \lambda^{*} \frac{\partial \dot{\lambda}}{\partial m} d \Gamma-\int_{\Gamma} \lambda \frac{\partial \lambda^{*}}{\partial m} d \Gamma-\frac{H}{\omega} \int_{\Omega} \lambda^{*} \dot{\lambda} d \Omega+\frac{1}{\omega} \int_{\Omega} \lambda^{*} \frac{\partial f}{\partial \dot{\boldsymbol{\sigma}}} \mathbf{C} \dot{\boldsymbol{\varepsilon}} d \Omega,
$$

onde $c$ é o termo livre que resulta da regularização da integral com o fluxo da solução fundamental.

Se em vez disso se tomasse o operador 


$$
-\left\llcorner\dot{\lambda}=-\omega \nabla^{2} \dot{\lambda}-H \dot{\lambda}=\frac{\partial f}{\sigma} \mathbf{C} \dot{\varepsilon}\right.
$$

para o caso de $H$ ser constante (como no encruamento isótropo linear com critério de von Mises), e tomando-se a solução fundamental correspondente, $\bar{\lambda}$, a representação integral resultaria em

$$
\bar{c} \lambda=\int_{\Gamma} \bar{\lambda} \frac{\partial \lambda}{\partial m} d \Gamma-\int_{\Gamma} \frac{\partial \bar{\lambda}}{\partial m} \lambda d \Gamma+\frac{1}{\omega} \int_{\Omega} \bar{\lambda} \frac{\partial f}{\partial \dot{\sigma}} \mathbf{C} \dot{\varepsilon} d \Omega .
$$

Conforme afirmado anteriormente, uma integral de domínio desaparece, mas a última permanece, forçando a discretização do domínio e a aproximação no interior. Em termos computacionais, a existência de um segundo termo de domínio não representa um esforço extra significativo.

A equação ( 4.52 ) ou ( 4.54 ) governa o campo do multiplicador plástico na região plástica do corpo, a qual pode se mover durante o processo de carregamento, conduzindo a um problema de contorno móvel.

Ainda é preciso estabelecer as condições de contorno para essa equação. Claramente, o multiplicador plástico deve ser nulo no contorno entre as regiões elástica e plástica. Resta o contorno externo do corpo, onde o multiplicador plástico tem valor positivo a ser determinado; portanto, seu fluxo deve ser prescrito, e parece natural que ele seja nulo, já que a região plástica não deve se movimentar para fora do domínio do corpo. Resumindo, a condições de contorno devem ser as seguintes:

$$
\begin{array}{ll}
\frac{\partial \dot{\lambda}}{\partial m}=0 & \text { no contorno do corpo } \\
\dot{\lambda}=0 & \text { no contorno da regiao plastica }
\end{array}
$$


Para lidar com o problema de potencial representado pelo multiplicador plástico pode ser preciso tomar cuidados especiais na abordagem numérica. É possível que haja deslocamento do contorno da região plástica apenas na direção externa desse contorno. Esse é o caso em que apenas uma região plástica monótona crescente está presente. Mas o esquema para identificar a região plástica precisa ser mais geral. O contorno plástico pode se mover na direção de seu interior. Ou então é possível que se encontrem pequenas regiões onde o multiplicador plástico se torne nulo. A fim de superar essas dificuldades, é proposto um outro procedimento que não requer que se encontre o contorno da região plástica no domínio. Para isso é preciso modificar a equação ( 4.52 ) de modo a tornar possível se prescrever potenciais em pontos internos. Isto é feito considerando-se uma 'força de volume' fictícia, $\dot{\overline{\mathbf{b}}}$, que fará o papel de campo conjugado. Essa força de volume fictícia não tem significado físico. Ela constitui a incógnita quando o potencial é prescrito no interior do domínio, mas seus valores não são utilizados diretamente na solução do problema da plasticidade com gradiente.

Após introduzir a força de volume fictícia, a equação( 3.30 ) torna-se

$$
\dot{\overline{\mathbf{b}}}+\frac{\partial f}{\partial \sigma} \mathbf{C} \dot{\varepsilon}-H \dot{\lambda}+\omega \nabla^{2} \dot{\lambda}=0
$$

Em conseqüência, a representação integral do potencial torna-se

$$
c \dot{\lambda}=\int_{\Gamma} \lambda^{*} \frac{\partial \dot{\lambda}}{\partial m} d \Gamma-\int_{\Gamma} \frac{\partial \lambda^{*}}{\partial m} \dot{\lambda} d \Gamma-\int_{\Omega} \frac{H}{\omega} \lambda^{*} \dot{\lambda} d \Omega+\frac{1}{\omega} \int_{\Omega} \lambda^{*} \frac{\partial f}{\partial \sigma} \mathbf{C} \dot{\boldsymbol{\varepsilon}} d \Omega+\int_{\Omega} \frac{1}{\omega} \lambda^{*} \dot{\overline{\mathbf{b}}} d \Omega .
$$

Note-se que a equação acima representa o multiplicador plástico também em pontos internos $(\operatorname{com} c=1)$. 


\section{Método dos elementos de contorno}

A formulação do método dos elementos de contorno para problemas elastoplásticos é apresentada neste ponto em sua forma mais usual. Sua extensão para a plasticidade não local (com o segundo gradiente do multiplicador plástico) também é formulada, e formas possíveis de sua implementação são discutidas. As peculiaridades da formulação do problema da plasticidade com gradiente pelo método dos elementos de contorno em comparação com a dos elementos finitos são brevemente comentadas. A discretização adotada na implementação do programa computacional é exposta.

\subsection{Plasticidade local}

Como se sabe, as equações ( 4.17 ) e ( 4.19 ) podem ser transformadas em representações algébricas, aproximando-se adequadamente $\dot{\mathbf{u}}$ e $\dot{\mathbf{p}}$ ao longo do contorno por elementos, assim como $\dot{\mathbf{b}}$ e $\dot{\sigma}$ podem ser divididos em células no domínio. O problema deve ser resolvido em sua forma incremental. Pode-se escrever tantas equações algébricas quanto necessário. De modo similar, pode-se escrever um número apropriado de equações algébricas para as tensões, aquelas nas quais os valores das tensões são necessárias para se resolver o problema. Assim, assumindo-se o domínio e contorno divididos em elementos e células, respectivamente, assim como funções de forma para a aproximação das variáveis, as equações ( 4.17 ) e ( 4.19 ) tornam-se

$$
[H][\dot{\boldsymbol{u}}]=[G][\dot{\boldsymbol{p}}]+[V][\dot{B}]+[\bar{E}]\left[\dot{\boldsymbol{\sigma}}^{p}\right]
$$




$$
[\dot{\boldsymbol{\sigma}}]=-\left[H^{l}\right][\dot{\boldsymbol{u}}]+\left[G^{l}\right][\dot{\boldsymbol{p}}]+\left[V^{l}\right][\dot{\boldsymbol{B}}]+\left[\bar{E}^{l}\right]\left[\dot{\boldsymbol{\sigma}}^{p}\right]
$$

onde $[\dot{\boldsymbol{u}}]$ e $[\dot{\boldsymbol{p}}]$ são vetores contendo os valores nodais para deslocamentos e reações, respectivamente; $[\dot{\sigma}]$ e $\left[\dot{\sigma}^{p}\right]$ são os vetores das tensões e das tensões iniciais; $[H],\left[\left[H^{l}\right],[G],\left[G^{l}\right],[V]\right.$ e $\left[V^{l}\right]$ são as matrizes de influência computadas pela integração de elementos e células.

Aplicando-se as condições de contorno, as equações anteriores reduzem-se a

$$
[A][X]=[F]+[\bar{E}]\left[\dot{\sigma}^{p}\right]
$$

$\mathrm{e}$

$$
[\dot{\sigma}]=-\left[A^{l}\right][X]+[X]+\left[V^{l}\right][\dot{B}]+\left[\bar{E}^{l}\right]\left[\dot{\sigma}^{p}\right],
$$

com $[A]$ e $\left[A^{l}\right]$ contendo os coeficientes devido às incógnitas no contorno; e $[F]$, $\left[V^{l}\right]$ são vetores independentes correspondentes aos valores prescritos no contorno e às forças de volume, respectivamente.

Também é bem conhecida a redução das equações anteriores a

$$
[X]=[M]+[R]\left[\dot{\sigma}^{p}\right]
$$

$\mathrm{e}$

$$
[\dot{\sigma}]=[N]+[S]\left[\dot{\sigma}^{p}\right]
$$

onde $[M]$ e $[N]$ contem a solução elástica devido aos valores prescritos; e $[R]$, $[S]$ contem as influências das tensões iniciais aplicadas.

Esta formulação pode ser aplicada na resolução de qualquer não linearidade que possa ser assumida como distribuída pelo domínio. Mediante uma modificação adequada, também pode ser utilizada na solução de problemas anisotrópicos. (Ver 
por exemplo CHAVES, FERNANDES e VENTURINI ${ }^{[16]}$ para a flexão de placas de espessura variável.)

Para se resolver um problema da plasticidade, deve-se condiderar a solução algébrica em termos de deslocamentos e tensões dadas pelas equações ( 5.5 )-( 5.6 ), lembrando-se de que as tensões plásticas devem ser calculadas a partir do multiplicador plástico governado pela equação ( 3.14 ). Assim, considerando-se que as equações matriciais ( 5.5 ) e ( 5.6 ) podem ser adotadas para um incremento e o multiplicador plástico é dado localmente pela equação ( 3.14 ), a presente formulação é adequada para a plasticidade clássica.

\subsection{Plasticidade com gradiente}

No caso da plasticidade com gradiente, o multiplicador plástico é obtido da equação ( 3.30 ) (ou ( 4.52 )) deve ser utilizada. Uma forma discretizada da expressão ( 3.30 ) também pode ser escrita, assumindo-se que os valores de contorno, $\dot{\lambda}$ e $\frac{\partial \dot{\lambda}}{\partial m}$, são aproximados ao longo dos elementos. Além disso, os domínios também podem ser escritos em termos algébricos dividindo-os em células e adotando funções de forma convenientes para a aproximação do multiplicador plástico $\dot{\lambda}$ e do termo independente $\frac{\partial f}{\partial \sigma} \mathbf{C} \dot{\varepsilon}$. A representação algébrica da equação ( 4.52 ) fica

$$
\left[H_{p}\right]\left[\dot{\lambda}_{\Gamma}\right]=\left[G_{p}\right]\left[\frac{\partial \dot{\lambda}_{\Gamma}}{\partial m}\right]-\left[\left[\bar{V}_{\Gamma}\right]\left[\left[\bar{V}_{\Omega}\right]\right]\left[\begin{array}{l}
{\left[\dot{\lambda}_{\Gamma}^{\cdot}\right]} \\
{\left[\dot{\lambda}_{\Omega}\right]}
\end{array}\right]+[V][\dot{B}],\right.
$$

onde os subscritos $\Gamma_{\Omega}$ e ${ }_{\Omega}$ aplicam-se a valores nodais no contorno e no domínio do corpo, respectivamente; $\left[H_{p}\right]$ e $\left[G_{p}\right]$ são as clássicas matrizes dos coeficientes para problemas de campo escalar para valores no contorno; [V] é a matriz correspondente 
ao campo de domínio, e $\left[\bar{V}_{\Gamma}\right]$ e $\left[\bar{V}_{\Omega}\right]$ correspondentes aos coeficientes do vetor de $\dot{\lambda}$ no contorno e no domínio, respectivamente.

Ressalte-se que, ao contrário do que ocorre na plasticidade local com encruamento isótropo, não é possível explicitar uma relação para o multiplicador plástico, de modo que o sistema de equações ( 5.7 ) precisa ser resolvido a cada iteração, antes que o problema não linear ( 5.6 ) possa ser utilizado.

No contexto do método dos elementos finitos, a plasticidade com gradiente tem sido formulada basicamente de duas maneiras. Numa das abordagens, utiliza-se uma forma fraca (resíduos ponderados) da condição de consistência $(\dot{f}=0)$ e o sistema de equações do multiplicador plástico é acoplado ao sistemas de equações de equilíbrio; o sistema resultante é resolvido, de modo que multiplicador plástico e equilíbrio são considerados simultaneamente (cf. de BORST e MUHLHAUS ${ }^{[12]} \mathrm{e}$ PAMIN ${ }^{[44]}$, como exemplos). Ressalte-se que neste caso o sistema de equações a resolver fica ampliado. Numa segunda abordagem, o problema original é transformado num equivalente, utilizando-se o conceito de variáveis generalizadas, e o multiplicador plástico pode então ser determinado, antes (em COMI e PEREGO ${ }^{[18]}$, ou DRIEMEIER ${ }^{[21]}$ por exemplo).

Ressalte-se que em ambas as abordagens o problema não linear é resolvido através de um método implícito, utilizando-se um operador tangente consistente ou similar.

Ainda considerando uma formulação explícita para a solução do problema não linear (o que corresponde à abordagem usual para elementos de contorno), podese utilizar a equação ( 4.57 ) para a representação algébrica. Neste caso, os valores do termo de volume fictício $\overline{\mathbf{b}}$ têm de ser aproximados pelas células de domínio. Onde os valores da variável de volume fictícia forem requeridos para a solução do problema, devem ser introduzidos os termos correspondentes, de modo que a expressão anterior torna-se 


$$
\begin{aligned}
{\left[H_{p}\right]\left[\dot{\lambda}_{\Gamma}^{\cdot}\right]=} & {\left[G_{p}\right]\left[\frac{\partial \dot{\lambda}_{\Gamma}}{\partial m}\right]-\left[\left[\bar{V}_{\Gamma}\right]\left[\bar{V}_{\Omega}\right]\right]\left[\begin{array}{c}
{\left[\dot{\lambda}_{\Gamma}^{\cdot}\right]} \\
{\left[\dot{\lambda}_{\Omega}\right]}
\end{array}\right] } \\
& +[V][\dot{B}]+[\bar{V}][\bar{B}] .
\end{aligned}
$$

$\mathrm{Na}$ medida em que se introduzem novos valores desconhecidos (no interior do domínio, $\left[\dot{\lambda}_{\Omega}\right]$ ), novas aplicações das representações integrais devem ser escritas para equilibrar o número de equações e incógnitas. Devem ser expressas relações do multiplicador plástico para os nós internos usados de modo a completar o conjunto final de equações:

$$
\begin{gathered}
{\left[\dot{\lambda}_{\Omega}\right]=-\left[H_{p}^{\Omega}\right]\left[\dot{\lambda}_{\Gamma}\right]+\left[G_{p}^{\Omega}\right]\left[\frac{\partial \dot{\lambda}_{\Gamma}}{\partial m}\right]-\left[\left[\bar{V}_{\Gamma}^{\Omega}\right]\left[\bar{V}_{\Omega}^{\Omega}\right]\right]\left[\begin{array}{c}
{\left[\dot{\lambda}_{\Gamma}\right]} \\
{\left[\dot{\lambda}_{\Omega}\right]}
\end{array}\right]} \\
+\left[V^{\Omega}\right][\dot{B}]+\left[\bar{V}^{\Omega}\right][\bar{B}],
\end{gathered}
$$

onde o sobrescrito ${ }^{\Omega}$ é utilizado para indicar que as matrizes assinaladas referem-se aos pontos de colocação internos.

As expressões anteriores, ( 5.8 ) e ( 5.9 ), podem ser reunidas numa só:

$$
\begin{aligned}
& {\left[\begin{array}{ll}
{\left[H_{p}\right]} & {[0]} \\
{\left[H_{p}^{\Omega}\right]} & {[I]}
\end{array}\right]\left[\begin{array}{l}
{\left[\dot{\lambda}_{\Gamma}^{\cdot}\right]} \\
{\left[\dot{\lambda}_{\Omega}\right]}
\end{array}\right]=\left[\begin{array}{l}
{\left[G_{p}\right]} \\
{\left[G_{p}^{\Omega}\right]}
\end{array}\right]\left[\begin{array}{l}
\left.\frac{\partial \dot{\lambda}_{\Gamma}}{\partial m}\right]
\end{array}\right]-\left[\begin{array}{cc}
{\left[\bar{V}_{\Gamma}\right]} & {\left[\bar{V}_{\Omega}\right]} \\
{\left[\bar{V}_{\Gamma}^{\Omega}\right]} & {\left[\bar{V}_{\Omega}^{\Omega}\right]}
\end{array}\right]\left[\begin{array}{l}
{\left[\dot{\lambda}_{\Gamma}^{\cdot}\right]} \\
{\left[\dot{\lambda}_{\Omega}\right]}
\end{array}\right]+} \\
& +\left[\begin{array}{c}
{[V]} \\
{\left[V^{\Omega}\right]}
\end{array}\right][\dot{B}]+\left[\begin{array}{c}
{[\bar{V}]} \\
{\left[\bar{V}^{\Omega}\right]}
\end{array}\right][\bar{B}]
\end{aligned}
$$


sendo [O] uma matriz nula e [I] a identidade.

$\mathrm{O}$ vetor $[\bar{B}]$ com os valores de força de volume para pontos internos é avaliado resolvendo-se o sistema acima para todos os pontos internos onde o multiplicador plástico é prescrito. Os valores obtidos, tanto quanto os valores do fluxo no contorno, não são diretamente utilizados na solução do problema da plasticidade, embora eles sejam considerados na resolução do problema do multiplicador plástico, que possui essa condição de contorno mista.

\subsubsection{Esquemas de solução e condições de contorno}

Vários esquemas podem ser adotados para a solução do sistema algébrico ou ( 5.10), com ou sem o termo de volume fictício $[\bar{B}]$ conforme discutido a seguir.

\subsubsection{Esquema 1}

No programa computacional foi implementado um esquema utilizando-se as equações do sistema ( 5.10 ) sem o termo de volume fictício $[\bar{B}]$, e considerando-se sua aplicação no corpo todo, ou seja, no domínio formado pela parte elástica e plástica em conjunto. As condições de contorno são as seguintes (ver Figura 5.1):

$$
\begin{aligned}
& \frac{\partial \dot{\lambda}_{\Gamma}}{\partial m}=0 \quad \text { em todo o contorno externo. } \\
&(\text { Prescritos } \dot{\lambda}=0 \text { no domínio elástico } \\
& \dot{B}=0 \text { no domínio elástico.) }
\end{aligned}
$$

O sistema de equações ( 5.10 ) neste caso reduz-se a 


$$
\left[\left[\begin{array}{cc}
{\left[H_{p}\right]} & {[0]} \\
{\left[H_{p}^{\Omega}\right]} & {[I]}
\end{array}\right]+\left[\begin{array}{cc}
{\left[\bar{V}_{\Gamma}\right]} & {\left[\bar{V}_{\Omega}\right]} \\
{\left[\bar{V}_{\Gamma}^{\Omega}\right]} & {\left[\bar{V}_{\Omega}^{\Omega}\right]}
\end{array}\right]\left[\begin{array}{c}
{\left[\dot{\lambda}_{\Gamma}\right]} \\
{\left[\dot{\lambda}_{\Omega}\right]_{p l}}
\end{array}\right]=\left[\begin{array}{c}
{[V]} \\
{\left[V^{\Omega}\right]}
\end{array}\right][\dot{B}],\right.
$$

onde o índice subscrito $p l$ em $\left[\dot{\lambda}_{\Omega}\right]_{p l}$ foi utilizado apenas para lembrar que somente os pontos de domínio da parte plástica precisam ser incorporados ao sistema de equações.

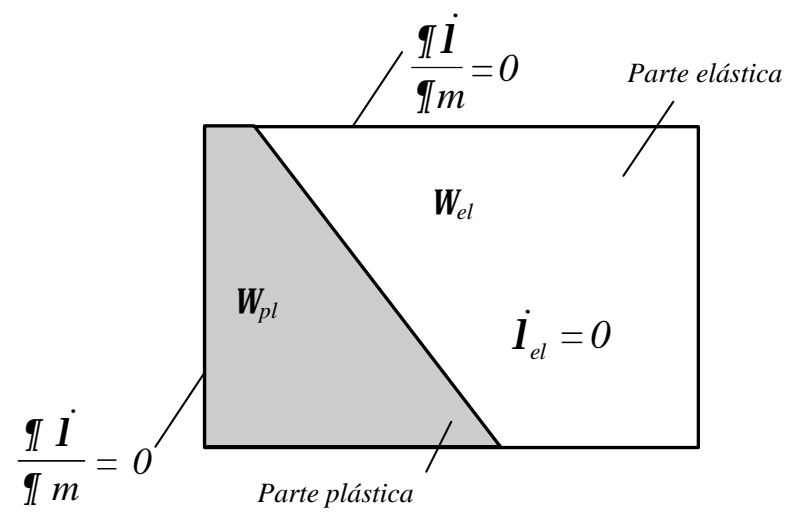

Figura 5.1 - Solução do multiplicador plástico: esquema 1.

Esta estratégia é a mais compacta e econômica. É necessário ter uma equação para cada ponto na região plástica, apenas. As matrizes de coeficientes precisam ser calculadas somente uma vez, sendo utilizados os elementos adequados na montagem do sistema com os dados de cada iteração.

Ressalte-se porém que a prescrição de valores para $\dot{\lambda}$ na região elástica, embora válida, não é estritamente correta. A formulação matemática do problema da plasticidade garante uma correção para possíveis erros no cálculo de $\dot{\lambda}$. E com a participação das equações de equilíbrio, eventuais desvios no cálculo de $\dot{\lambda}$ podem ser corrigidos durante o processo iterativo. Mas embora possibilite a obtenção de resultados corretos, esse esquema não pode ser considerado como inteiramente consistente. A inclusão do termo de volume fictício, a fim de se prescreverem os valores de $\dot{\lambda}$ na parte elástica do domínio, parece mais consistente, mesmo se os 
reflexos desses termos nos resultados não forem necessariamente significativos. Embora esse ponto não tenha sido investigado neste trabalho, constitui um tópico que demanda um estudo mais detalhado.

\subsubsection{Esquema 2}

Um segundo esquema pode ser proposto para permitir a prescrição de $\dot{\lambda}=0$ na parte elástica do corpo, para a solução do sistema. Para isso, o termo de volume fictício $[\bar{B}]$ é considerado. As condições de contorno são as seguintes:

$$
\begin{aligned}
\frac{\partial \dot{\lambda}_{\Gamma}}{\partial m}=0 \quad & \text { em todo o contorno externo. } \\
\text { (Prescritos } \dot{\lambda}= & =\dot{B} \text { na parte elástica, } \\
\dot{\lambda} & =0 \text { na interface elastoplástica, } \\
& \bar{B}=0 \text { na parte plástica restante.) }
\end{aligned}
$$

O sistema algébrico resultante é

$$
\begin{aligned}
& {\left[\left[\begin{array}{cc}
{\left[H_{p}\right]} & {[0]} \\
{\left[H_{p}^{\Omega}\right]} & {[I]}
\end{array}\right]+\left[\begin{array}{ll}
{\left[\bar{V}_{\Gamma}\right]} & {\left[\bar{V}_{\Omega}\right]} \\
{\left[\bar{V}_{\Gamma}^{\Omega}\right]} & {\left[\bar{V}_{\Omega}^{\Omega}\right]}
\end{array}\right]\left[\begin{array}{c}
{\left[\dot{\lambda}_{\Gamma}\right]} \\
{\left[\dot{\lambda}_{\Omega}\right]_{p l}}
\end{array}\right]=\right.} \\
& =\left[\begin{array}{c}
{[V]} \\
{\left[V^{\Omega}\right]}
\end{array}\right][\dot{B}]+\left[\begin{array}{c}
{[\bar{V}]} \\
{\left[\bar{V}^{\Omega}\right]}
\end{array}\right][\bar{B}],
\end{aligned}
$$




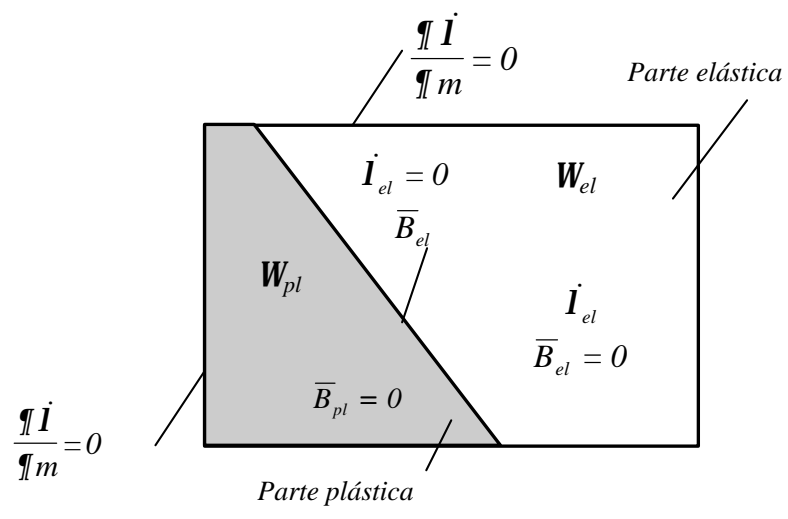

Figura 5.2 - Solução do multiplicador plástico: esquema 2.

Uma desvantagem nesta estratégia é que o problema do multiplicador plástico precisa ser resolvido para o domínio todo sempre, ou seja, uma equação para cada ponto nodal no domínio inteiro. Embora não tenha sido feito neste trabalho, seria interessante investigar se essa estratégia pode trazer ganhos significativos de precisão ou correção de resultados, em diferentes situações de carregamento, de modo a compensar esse aumento de custo computacional.

\subsubsection{Esquema 3}

Uma terceira alternativa consiste em se considerar apenas a região plástica para a solução do sistema ( $\mathbf{5 . 1 0}$ ), sem o termo de volume fictício $[\bar{B}]$. Esta estratégia, que não chegou a ser implementada neste trabalho, envolve um algorítmo para atualização do domínio plástico, a cada iteração. As condições de contorno, neste caso, são mais intuitivas:

$$
\begin{array}{ll}
\frac{\partial \dot{\lambda}_{\Gamma}}{\partial m}=0 & \text { no contorno externo. } \\
\dot{\lambda}_{\Gamma}=0 & \text { no contorno interno da região plástica. }
\end{array}
$$




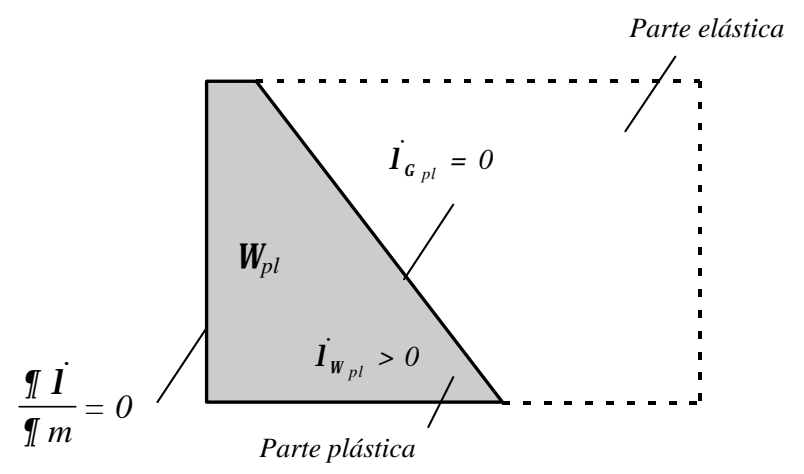

Figura 5.3 - Solução do multiplicador plástico: esquema 3.

Esta estratégia exige a construção de um algorítmo para a determinação da região elástica, a cada iteração. Uma segunda observação é que as matrizes dos coeficientes tem de ser computada para cada iteração, de acordo com o domínio da região plástica corrente. Apesar do aumento do esforço computacional, ao menos parte dele pode ser compensado pelo fato de que o domínio, ou seja, discretização e solução do problema, envolve apenas a parte plástica em cada iteração.

Uma observação interessante a fazer é que, após a determinação do multiplicador plástico $\dot{\lambda}$, seu segundo gradiente, $\nabla^{2} \dot{\lambda}$ pode ser diretamente determinado retornando-se com o valor $\dot{\lambda}$ na equação de consistência $(\dot{f}=0)$ para cada ponto. Portanto, não há necessidade de se obter o gradiente a partir da derivação das funções de forma para $\dot{\lambda}$, que exigiria um grau maior para essas funções de forma - como se faz por elementos finitos, na formulação (cf. PAMIN ${ }^{[44]}$ ) por resíduos ponderados para o campo $\lambda^{\bullet}$. É suficiente utilizar aproximação linear para $\lambda^{\bullet}$ como na formulação por variáveis generalizadas (cf. COMI e PEREGO ${ }^{[18]}$ ). Outra observação é que na formulação aqui proposta, as condições de contorno são claramente determinadas - na formulação por resíduos ponderados há necessidade de escolha entre duas condições igualmente admissíveis. 


\subsection{Algorítmo para solução}

Segue um resumo do algorítmo de tensão inicial. Um resumo mais completo é apresentado no capítulo seguinte, utilizando-se a formulação implícita para o método dos elementos de contorno.

Devido à natureza incremental do problema plástico, a equação ( 5.10 ) representa uma forma incremental, $\Delta \lambda$. Para um incremento de carga, o problema é resolvido elasticamente, e o incremento elástico $[N]$ é adicionado à tensão atual. Para pontos que atingem o estágio plástico, o incremento é computado a partir da lei de Hooke (( 4.13 )-c). Observe-se que o incremento de deformação plástica é dado por ( 3.10 ); $\Delta \lambda$ vem de ( 3.14 ) no caso da plasticidade local, ou de ( 3.30 ) na plasticidade com gradiente. O incremento de tensão atual é a diferença entre os incrementos de tensão plástica $\left(\mathbf{C} \Delta \boldsymbol{\varepsilon}^{p}\right)$ e "elástica" $(\mathbf{C} \Delta \boldsymbol{\varepsilon})$. Se os valores do incremento plástico são suficientemente pequenos (de acordo com uma tolerância especificada), toma-se um novo incremento de carga. Caso contrário, inicia-se uma nova iteração, tomandose como novo incremento elástico o produto da matriz [S] (equação ( 5.6 )) pelo incremento de tensão plástica.

\subsection{Discretização e implementação}

As três versões (explícito local, com gradiente, e implícito local) que contribuem com os exemplos numéricos apresentados neste trabalho referem-se à plasticidade bidimensional, plasticidade associada com encruamento isótropo linear e critério de von Mises.

Todas as versões utilizam os mesmos elementos de contorno, retos, com interpolação linear para deslocamentos, tensão e multiplicador plástico (na formulação com gradiente). As células, triangulares, apresentam também interpolações linerares para todos os campos.

Devido ao emprego da representação integral de tensão no contorno, conforme o método descrito no capítulo anterior, era necessário ter continuidade da primeira derivada dos deslocamentos nos pontos singulares. A fim de se manter a 
interpolação e elementos lineares, optou-se pela utilização de nós duplos em todos os elementos de contorno, situados das extremidades sempre da distância de um quarto do comprimento do elemento. Essa formulação, embora satisfatória para avaliação geral do programa, só pode ser considerada como provisória, por representar uma estratégia pouco natural e com certo desperdício de recursos.

Para melhorar a precisão dos coeficientes das matrizes na proximidade dos pontos singulares, foram utilizados subelementos no contorno. Essa técnica consiste em dividir-se os elementos para a integração numérica quando os pontos de integração encontram-se próximos do ponto singular. Os elementos são divididos de acordo com sua proximidade do ponto de colocação: Quanto mais próximos, menores os subelementos. Dessa forma evita-se que a distância entre os pontos de integração e o singular tornem-se comparáveis ao tamanho do elemento, como referência do intervalo de integração numérica.

Os resultados globais das construções das matrizes de coeficientes são bons, normalmente próximos do limite de precisão da máquina (com dupla precisão). Nos pontos mais críticos nos exemplos maiores, a precisão parece não ficar abaixo de $10^{-}$ ${ }^{9}$ ou muito próximo disso. Mas normalmente ficam mesmo na ordem de $10^{-17}$ a $10^{-14}$.

Segue uma conclusão referente à implementação realizada.

Várias tentativas para se melhorar o desempenho do programa foram realizadas. Algumas exigindo um esforço maior, como a obtenção e consequente utilização da representação integral exata da tensão no contorno, ou a solução do problema não linear discretizado utilizando-se o operador tangente consistente. Outras alterações de caráter mais simples, como alterações na precisão ou número de pontos de Gauss, ou a utilização de subelementos dividindo os elementos originais em partes menores, na proporção da proximidade do ponto singular. Ou ainda a utilização de bibliotecas numéricas para melhora do desempenho. 
Na verdade, a maior parte do tempo empregado neste trabalho foi, de longe, gasto com a implementação, avaliação, e melhoras de desempenho do programa computacional.

Partiu-se de um programa inicial construído pelo orientador deste trabalho. Todas as rotinas sofreram alterações, muitas delas bastante significativas, algumas novas ou completamente alteradas.

Apesar de o programa inicial apresentar um bom desempenho para problemas habituais de plasticidade, desde o início encontraram-se dificuldades para a solução de problemas mais exigentes, requisitando maior precisão, convergência, ou capacidade de procesamento.

O programa inicial, codificado em Fortran 77, aos poucos ganhou os principais aspectos da norma Fortran 90, nos pontos mais importantes.

Foi escohido um gerador de malhas de licença pública: o "Triangle", de

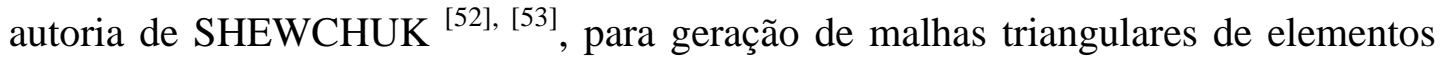
finitos, e então foi construída uma rotina para efetuar a renumeração da malha para o esquema de elementos de contorno adotado, o que incluiu a inserção de novos nós no contorno (foram utilizados nós duplos no contorno).

Também foi construído um programa visual, que por sua vez utilizou um programa específico para a construção e manipulação de dados científicos (Data Explorer, IBM). A utilização de visualização mostrou-se essencial para a análise dos resultados, conforme esperado.

Outro ponto importante para o programa foi a utilização de bibliotecas numéricas (no caso, a NAG) para as soluções dos sistemas de equações lineares, trazendo melhoria do desempenho.

O desempenho também foi trabalhado, conseguindo-se significativa melhoria com o emprego de otimizações de compilação.

A capacidade de processamento foi significativamente ampliada.

No final o programa parece satisfatoriamente robusto, com boa estabilidade numérica, capacidade e velocidade de cálculo também boas. Foram utilizados supercomputadores. 
Com respeito à aproximação, agora.

A utilização de funções de forma de grau mais elevado que as interpolações lineares utilizadas seria provavelmente interessante. Essa possibilidade foi considerada, mas não chegou a ser implementada (apenas a utilização de interpolação constante chegou a ser realizada).

Uma versão com tensão constante nas células foi desenvolvida, embora não tenha se mantido ativa, precisando ser atualizada para a atual versão do programa.

Uma versão contendo uma formulação implícita utilizando um esquema na linha proposta por TELLES e CARRER ${ }^{\text {[54], [55] }}$ também foi desenvolvida, tendo sido testada e comparada com as outras utilizadas nos exemplos numéricos aqui apresentados.

Com respeito à utilização de representação integral para as tensões, apesar de interessante do ponto de vista teórico, não foi analisado quanto os resultados foram realmente afetados por essa formulação - embora seja provável ter sido obtido um ganho significativo. Uma investigação sistemática dessa alternativa em comparação com a avaliação numérica habitual (derivação das funções de forma do deslocamento) seria bastante interessante. Sabe-se que no caso da elasticidade a melhora dos resultados é extremamente significativa, em certos casos. POON et al. [46] sugerem benefícios para seu emprego na plasticidade e declaram estar trabalhando em sua implementação.

Quanto à formulação utilizando o operador tangente consistente, discutido no capítulo seguinte, talvez seja o aspecto mais positivo dentre os realizados, em termos de resultados. Mesmo a utilização do algorítmo de retorno radial isolado, sem a aplicação do método de Newton, já parece trazer vantagens significativas, possibilitando um programa mais compacto computacional e um código mais claro. 


\section{Formulação implícita com operador tangente consistente}

Foram implementadas duas formulações implícitas para a solução do problema elastoplástico (para a plasticidade com gradiente não se chegou a implementar a formulação do algorítmo implícito). A primeira de acordo com a proposta de TELLES e CARRER $(1991,1994)^{[54], ~[55], ~ a ~ s e g u n d a ~ u t i l i z a n d o ~ o ~}$ operador tangente consistente, de forma similar a BONNET e MUKHERJEE (1996). ${ }^{[9]}$, porém fazendo sua adaptação ao algorítmo de tensão inicial. Mas somente a segunda formulação foi suficientemente testada e foi a única utilizada nos exemplos.

No modelo elastoplástico do contínuo clássico, a evolução da tensão pode ser definida, a partir de um estado inicial $\sigma^{\mathbf{0}}$, como

$$
\sigma=\sigma^{0}+\int \mathbf{C}^{\mathrm{ep}} d \varepsilon
$$

com o operador tangente contínuo dado por

$$
\mathbf{C}^{\mathrm{ep}}=\frac{\partial \sigma}{\partial \varepsilon}
$$

No entanto, para o algorítmo de resolução não linear, a integração é incremental para a deformação $(\Delta \varepsilon)$. Portanto, (conforme proposto inicialmente por SIMO e TAYLOR ${ }^{[51]}$ ) o operador tangente deve ser consistente com o algorítmo do incremento: 


$$
\mathbf{C}^{\mathrm{epC}}=\frac{\partial \sigma}{\partial \Delta \varepsilon}
$$

ou seja, a construção do operador tangente deve ser feita de acordo com a relação algorítmica existente entre o incremento de tensão e o de deformação.

Os operadores coincidem para o incremento infinitesimal, mas podem diferir significativamente, especialmente para incrementos maiores. (No processo incremental, de qualquer forma, há um erro envolvido numa utilização do operador tangente contínuo.

Telles e Carrer propõem um algorítmo implícito em que o operador tangente provém dos incrementos de modo independente do modo como ocorre o retorno da tensão à superfície de plastificação, fundamentando-se numa formulação com operador de rigidez tangente contínuo. Bonnet e Mukherjee utilizaram pela primeira vez no contexto dos elementos de contorno o conceito de operador tangente consistente algorítmico, utilizando uma formulação para deformações iniciais.

Ao contrário de Bonnet e Mukherjee, a formulação implementada neste trabalho é de tensões iniciais, de modo que foi necessário obter as relações adequadas para esse caso. Uma breve descrição é dada abaixo.

Uma primeira fase consiste em se introduzir uma formulação implícita para se obter o retorno radial para as tensões durante a plastificação. Na plasticidade local com critério de von Mises e encruamento linear descritos aqui isso é simples, mas outros casos podem ser generalizados a partir desse, sem grandes dificuldades. Uma etapa de previsão considerando o incremento de carga como inteiramente elástico é utilizada como uma tentativa inicial, que então é corrigida num segundo passo (etapa de correção), de modo que o retorno é feito radialmente.

Esse algorítmo de retorno é então utilizado para se obter o operador de rigidez tangente local que será utilizado na correção da rigidez global de modo consistente.

O algorítmo de retorno radial é obtido da maneira descrita a seguir.

A equação ( 5.6), somada de $\left[\sigma^{p}\right]$ de cada lado, pode ser reescrita (em forma incremental), como 


$$
\left[Y\left(\Delta \varepsilon_{n}\right)\right]=-[I][C]\left[\Delta \varepsilon_{n}\right]+\left[N_{n}\right]+[\bar{S}]\left[[C]\left[\Delta \varepsilon_{n}\right]-\left[\Delta \sigma_{n}\right]\right]=0
$$

com [C] representando o tensor de rigidez elástico e [I] a matriz identidade para tornar o operador global, $([\bar{S}]=[S]+[I]), \Delta$ o incremento, o subscrito ${ }_{n} \mathrm{o}$ enésimo incremento, e $\Delta \varepsilon_{n}, \Delta \sigma_{n}$ dados por

$$
\begin{gathered}
\varepsilon_{n+1}=\varepsilon_{n}+\Delta \varepsilon_{n} \\
\sigma_{n+1}^{\text {arr }}=\sigma_{n}+\Delta \sigma_{n},
\end{gathered}
$$

onde $\sigma_{n}^{\text {arr }}\left(\Delta \varepsilon_{n}\right)$ é definido pelo algorítmo de retorno utilizado, e $\sigma_{n}$ é o fornecido pela equação do método dos elementos de contorno.

Substituindo-se ( 6.5 )-b em ( 6.4 ), tem-se

$$
\begin{aligned}
{\left[Y\left(\Delta \varepsilon_{n}\right)\right]=} & -[I][C]\left[\Delta \varepsilon_{n}\right]+\left[N_{n}\right] \\
& +[\bar{S}]\left[[C]\left[\Delta \varepsilon_{n}\right]-\left[\sigma_{n}^{\mathrm{arr}}\right]+\left[\sigma_{n}\right]\right]=0^{\circ}
\end{aligned}
$$

Uma maneira de se resolver a equação não linear acima é através do método de Newton. Nesta solução iterativa utiliza-se a seguinte definição:

$$
\Delta \varepsilon_{n}^{i+1}=\Delta \varepsilon_{n}^{i}+\delta \Delta \varepsilon_{n}^{i}
$$

onde ${ }^{i}$ indica a iteração i.

O método de Newton estabelece que 


$$
\left[Y\left(\Delta \varepsilon_{n}^{i}\right)\right]+\frac{\partial\left[Y\left(\Delta \varepsilon_{n}^{i}\right)\right]}{\partial \Delta \varepsilon_{n}^{i}} \delta \Delta \varepsilon_{n}^{i}+\ldots=0
$$

Utilizando-se apenas a primeira variação de $\left[Y\left(\Delta \varepsilon_{n}{ }^{i}\right)\right]$, resulta

$$
\left[Y\left(\Delta \varepsilon_{n}^{i}\right)\right]=\left[[I][C]-[\bar{S}]\left[[C]-\left[\partial \sigma_{n+1}^{\operatorname{arr} i} / \partial \Delta \varepsilon_{n}^{i}\right]\right]\right]\left[\delta \Delta \varepsilon_{n}^{i}\right]
$$

onde o termo que multiplica o vetor de deformação é o operador tangente consistente para a formulação elastoplástica para o método dos elementos de contorno.

\subsection{Operador tangente consistente para o algorítmo de tensão inicial, estado plano de tensão, encruamento isótropo linear com critério de von Mises}

No programa computacional implementado para a obtenção dos exemplos, foi desenvolvida uma formulação do operador tangente consistente para o algorítmo de tensão inicial no caso do estado plano de tensão. O operador tangente consistente parece ter sido utilizado no método dos elementos de contorno apenas por Bonnet e seus colaboradores, a implementação de um caso plano ter sido implementada apenas em seu trabalho mais recente. Bonnet utiliza o algorítmo de deformação inicial, que possibilita uma aplicação mais direta do operador tangente. Uma formulação para o caso de tensão inicial é apresentada a seguir, para o caso de encruamento isótropo linear com critério de plastificação von Mises, tensão inicial e estado plano de tensão. Uma extensão dessa formulação para o gradiente plástico será discutida noutra seção.

O primeiro passo consiste na escolha de um adequado algorítmo de retorno para a superfície plástica. Isto é feito através do procedimento de tipo implícito descrito a seguir, dividido em uma etapa de previsão e outra de correção. 
Para o encruamento isótropo linear, o critério de plastificação de von Mises estabelece que

$$
f_{n+1}=\sqrt{\frac{3}{2}}\left\|\mathbf{S}_{n+1}\right\|-\sigma_{0}-h \lambda_{n+1} \leq 0,
$$

onde $\sigma_{0}$ representa o limite elástico inicial, $h$ é o módulo plástico (inclinação da curva tensão por deformação plástica), $\lambda$ é o multiplicador plástico, $\mathbf{S}$ a tensão desviadora, e o índice ${ }_{n}$ representa o enésimo passo de carga.

No incremento $n+1$, na etapa de previsão admite-se um estado de tentativa, sendo dado como conhecido o estado no incremento anterior, n. Nesse estado de tentativa admite-se que o incremento é elástico, isto é,

$$
\begin{gathered}
\Delta \lambda_{n}{ }^{t}=\lambda_{n+1}-\lambda_{n}=0, \\
\boldsymbol{\varepsilon}_{n+1}^{p}{ }^{t}=\boldsymbol{\varepsilon}_{n}^{p}, \\
\boldsymbol{\sigma}_{n+1}^{t}=\mathbf{C}\left(\boldsymbol{\varepsilon}_{n+1}{ }^{t}-\boldsymbol{\varepsilon}_{n}^{p}\right),
\end{gathered}
$$

onde o índice ${ }^{t}$ indica o estado de tentativa, $\varepsilon^{p}$ o tensor de deformação plástica acumulada, e $\mathbf{C}$ o tensor de rigidez elástica.

Utilizando-se as relações para tensão desviadora ( 2.8 ), do tensor de rigidez elástico C (equação ( 3.2 ) )e observando-se que $e^{p}=t r \varepsilon^{p}=0$ (a plastificação não produz alteração de volume), a tensão desviadora para o estado de tentativa no incremento $\mathrm{n}+1$ pode ser escrita como:

$$
\mathbf{S}_{n+1}{ }^{t}=2 \mu\left(e_{n+1}-\varepsilon_{n}^{p}\right),
$$


com $e=\operatorname{tr}\left(\varepsilon_{n+1}\right), \mu$ a constante de Lamé ( $\mu=G$, sendo $G$ o módulo de deformação transversal).

A tensão desviadora no incremento $n+1$ pode então ser escrita como uma correção a partir do estado de tentativa:

$$
\begin{aligned}
\mathbf{S}_{n+1} & =2 \mu\left(e_{n+1}-\left(\boldsymbol{\varepsilon}_{n}^{p}+\Delta \boldsymbol{\varepsilon}_{n}^{p}\right)\right) \\
& =2 \mu\left(e_{n+1}-\varepsilon_{n}^{p}\right)-2 \mu \Delta \lambda_{n} \mathbf{n}, \\
& \quad \text { ou } \\
\mathbf{S}_{n+1} & =\mathbf{S}_{n+1}{ }^{t}-2 \mu \Delta \lambda_{n} \mathbf{n},
\end{aligned}
$$

onde $\Delta \varepsilon_{n}^{p}=\varepsilon_{n+1}-\varepsilon_{n}$ vem de (6.7), com

$$
\mathbf{n}=\frac{\partial F}{\partial \sigma}=\frac{\partial f}{\partial \sigma}
$$

admitindo-se plasticidade associada $(F=f)$. Substituindo-se $f$ de ( 6.10 ) na expressão anterior, obtém-se

$$
\begin{gathered}
\mathbf{n}_{n+1}=\frac{\partial f_{n+1}}{\partial \sigma_{n+1}}=\sqrt{\frac{3}{2}} \frac{\mathbf{S}_{n+1}}{\left\|\mathbf{S}_{n+1}\right\|}, \\
\left\|\mathbf{n}_{n+1}\right\|=\sqrt{\frac{3}{2}} .
\end{gathered}
$$

Observe-se que a equação ( 6.13 ) pode ser escrita como

$$
\mathbf{S}_{n+1}{ }^{t}=\left(\frac{\left\|\mathbf{S}_{n+1}\right\|}{\left\|\mathbf{n}_{n+1}\right\|}+2 \mu \Delta \lambda_{n}\right) \mathbf{n},
$$


o que mostra que $\mathbf{S}_{n=1}{ }^{t}$ e $\mathbf{S}_{n=1}$ estão alinhados (com $\mathbf{n}$ ).

Para pontos sob o regime plástico, pode-se então rescrever $f_{n+1}$ como

$$
f_{n+1}=\sqrt{\frac{3}{2}}\left(\mathbf{S}_{n+1}{ }^{t}-2 \mu \Delta \lambda_{n} \mathbf{n}_{n+1}\right) \frac{\mathbf{n}_{n+1}}{\left\|\mathbf{n}_{n+1}\right\|}-\sigma_{0}-h \lambda_{n}-h \Delta \lambda_{n}=0
$$

o que permite a obtenção do multiplicador plástico $\Delta \lambda_{n}$ :

$$
\Delta \lambda_{n}=\frac{\sqrt{\frac{3}{2}} \mathbf{S}_{n+1}{ }^{t} \frac{\mathbf{n}_{n+1}}{\left\|\mathbf{n}_{n+1}\right\|}-\sigma_{0}-h \lambda_{n}}{3 \mu+h}=\frac{f_{n+1}{ }^{t}}{3 \mu+h} .
$$

Conhecido o multiplicador plástico, é possível avançar na direção da obtenção de outro resultado necessário para a construção dessa formulação implícita: a obtenção do operador tangente local ou tensor elastoplástico algorítmico, que neste caso pode ser derivado analiticamente a partir de $\sigma_{n+1}$ :

$$
\mathbf{C}_{n+1}^{e p}=\frac{\partial \sigma_{n+1}}{\partial \Delta \varepsilon_{n}}=\frac{\partial\left(\mathbf{C}\left(\varepsilon_{n}+\Delta \varepsilon_{n}-\varepsilon_{n}^{p}-\Delta \lambda_{n} \mathbf{n}_{n+1}\right)\right)}{\partial \Delta \varepsilon_{n}} .
$$

Com a expressão para o tensor de rigidez elástico (equação( 3.2 )) e observando-se que $\operatorname{tr}\left(\mathbf{n}_{n+1}\right)=0$, a expressão acima pode ser calculada. Lembrando-se que $(\nabla(a \mathbf{m})=a \nabla \mathbf{m}+\mathbf{m} \otimes \nabla a)$, obtém-se

$$
\mathbf{C}_{n+1}^{e p}=\frac{\partial \sigma_{n+1}}{\partial \Delta \varepsilon_{n}}=\mathbf{C}-2 \mu\left(\Delta \lambda_{n} \frac{\partial \mathbf{n}_{n+1}}{\partial \Delta \varepsilon_{n}}+\mathbf{n}_{n+1} \otimes \frac{\partial \Delta \lambda_{n}}{\partial \Delta \varepsilon_{n}}\right)
$$


Resolvendo-se as derivadas e adotando-se a mesma notação de PROENÇA ${ }^{[47]}$, a expressão para $\mathbf{C}_{n+1}^{\mathbf{e p}}$ pode ser escrita como

$$
\begin{gathered}
\mathbf{C}_{n+1}^{e p}=\frac{\partial \sigma_{n+1}}{\partial \Delta \varepsilon_{n}}=\mathbf{C}-2 \mu \theta_{n+1}\left(\mathbf{I I}-\frac{1}{3} \mathbf{I} \otimes \mathbf{I}\right)-2 \mu \overline{\theta_{n+1}}\left(\mathbf{n}_{n+1} \otimes \mathbf{n}_{n+1}\right), \\
\operatorname{com} \quad \theta_{n+1}=2 \mu \frac{\Delta \lambda_{n}}{\left\|\mathbf{S}_{n+1}\right\|} \\
e \quad{\overline{\theta_{n+1}}}_{\bar{y}^{\prime}}=\left(\frac{1}{1+\frac{h}{3 \mu}}\right)-\theta_{n+1} .
\end{gathered}
$$

É interessante observar na expressão anterior que $\mathbf{C}_{n+1}^{\text {ep }}$ (algorítmo) tende a $\mathbf{C}^{\text {ep }}$ (contínuo) quando $\Delta \lambda_{n}$ tende a zero $\left(\theta_{n+l} \rightarrow 0\right)$, ou seja quando o incremento tende a zero $(\Delta t \rightarrow 0)$. Como o proceso é incremental, a adoção do operador contínuo implicaria na supressão desses termos (em $\theta_{n+1}$ ), acarretando numa diferença tanto maior quanto maior o passo de carga utilizado.

\subsubsection{Estado plano de tensão, algorítmo de tensão inicial}

Segue a particularização da formulação acima para o caso de tensão plana com a plasticidade introduzida através do algorítmo de tensão inicial.

Os tensores de tensão e de deformação no estado plano de tensão são 


$$
[\sigma]=\left[\begin{array}{ccc}
\sigma_{11} & \sigma_{12} & 0 \\
\sigma_{21} & \sigma_{22} & 0 \\
0 & 0 & 0
\end{array}\right], \quad[\varepsilon]=\left[\begin{array}{ccc}
\varepsilon_{11} & \varepsilon_{12} & 0 \\
\varepsilon_{21} & \varepsilon_{22} & 0 \\
0 & 0 & \frac{-\lambda_{c}\left(\varepsilon_{11}+\varepsilon_{22}\right)}{2 \mu+\lambda_{c}}
\end{array}\right] \text {, }
$$

sendo $\lambda_{c}$ e $\mu$ as constantes elásticas de Lamé.

Em forma indicial, a expressão ( 6.21 ) do operador tangente local escreve-se como

$$
C_{n+l(i j k l)}^{e p}=C_{i j k l}-2 \mu \theta_{n+l}\left(I I_{i j k l}-\frac{1}{3} I_{i j} I_{k l}\right)-2 \mu \overline{\theta_{n+l}}\left(n_{n+1(i j)} n_{n+1(k l)}\right)
$$

Levando-se em conta esses resultados, considere-se novamente a expressão ( 6.9 ) da formulação do método dos elementos de contorno utilizando o operador tangente consistente:

$$
\left[Y\left(\Delta \varepsilon_{n}^{i}\right)\right]=\left[[C]-[\bar{S}]\left[[C]-\left[C_{n+1}^{e p} i\right]\right]\right]\left[\delta \Delta \varepsilon_{n}^{i}\right]
$$

onde $\left[C_{n+1}^{e p i}\right]$ é obtido agrupando-se adequadamente os operadores $C_{n+1}^{e i^{i}}$ locais determinados pela expressão mais acima. (O índice sobrescrito $i$ representa a iésima iteração.)

Neste ponto é conveniente observar que, no algorítmo de tensão inicial é preciso reaplicar o resíduo em forma de tensão. Isto é feito obtendo-se a tensão inicial multiplicando-se os valores de deformação encontrados da equação anterior pelo tensor de rigidez elástico $(\mathbf{C} \delta \Delta \varepsilon$ ) (que significa o sentido inverso do que foi utilizado na obtenção de ( 6.8 ) a partir de ( 5.6 ) ). 
Outro aspecto a destacar é que na solução do sistema de equações ( 6.24 ) foi utilizada uma propriedade daquelas matrizes que possibilita reduzir o número de incógnitas somente ao número de pontos no regime plástico na iteração considerada. Conforme observado por BONNET e MUKHERJEE ${ }^{[9]}$, é possível decompor o sistema em duas partes, resolvendo uma delas e obtendo a segunda a partir da primeira. Reescrevendo-se o sistema como

$$
\begin{gathered}
{\left[Y\left(\Delta \varepsilon_{n}^{i}\right)\right]=\left[\left[\bar{C}_{n+1}^{i}\right]\right]\left[\delta \Delta \varepsilon_{n}^{i}\right],} \\
\operatorname{com} \\
\left.\left[\bar{C}_{n+1}^{i}\right]\right]=\left[[C]-[\bar{S}]\left[[C]-\left[C_{n+1}^{e p} i\right]\right]\right],
\end{gathered}
$$

o sistema pode ser decomposto como

$$
\left[\begin{array}{cc}
{\left[\bar{C}_{n+1}^{i}\right]_{P P}} & {\left[\bar{C}_{n+1}^{i}\right]_{P E}} \\
{\left[\bar{C}_{n+1}^{i}\right]_{E P}} & {\left[\bar{C}_{n+1}^{i}\right]_{E E}}
\end{array}\right]\left[\begin{array}{l}
{\left[\delta \Delta \varepsilon_{n}^{i}\right]_{P}} \\
{\left[\delta \Delta \varepsilon_{n}^{i}\right]_{E}}
\end{array}\right]=\left[\begin{array}{l}
{\left[Y\left(\Delta \varepsilon_{n}^{i}\right)\right]_{P}} \\
{\left[Y\left(\Delta \varepsilon_{n}^{i}\right)\right]_{E}}
\end{array}\right],
$$

onde os índices subscritos $P, E$ referem-se aos pontos no estado plástico e elástico, respectivamente, na iteração $i$.

Observando-se que $\mathbf{C}_{n+1}^{e p ~}{ }^{i}=\mathbf{C}$ para todos os pontos em regime elásticos e que a matriz elástica global $[C]$ é quase diagonal, tem-se que

$$
\left[\bar{C}_{n+1}^{i}\right]_{P E}=0 \quad e \quad\left[\bar{C}_{n+1}^{i}\right]_{E E}=[C]
$$

de modo que o sistema de equações ( 6.26 ) pode ser resolvido apenas para os pontos plásticos na iteração: 


$$
\left[\bar{C}_{n+l}^{i}\right]_{P P}\left[\delta \Delta \varepsilon_{n}^{i}\right]_{P}=\left[Y\left(\Delta \varepsilon_{n}^{i}\right)\right]_{P}
$$

sendo os valores para os pontos elásticos obtidos posteriormente, a partir dos plásticos:

$$
\left[\bar{C}_{n+1}^{i}\right]_{E E}\left[\delta \Delta \varepsilon_{n}^{i}\right]_{E}=\left[Y\left(\Delta \varepsilon_{n}^{i}\right)\right]_{E}-\left[\bar{C}_{n+1}^{i}\right]_{E P}\left[\delta \Delta \varepsilon_{n}^{i}\right]_{P}
$$

o que acarreta numa considerável redução do esforço computacional, quando o número de pontos em regime elástico na iteração é significativo.

Registre-se que na implementação computacional, a correção da rigidez foi utilizada em todas iterações.

\subsubsection{Esquema do algorítmo computacional}

O algorítmo computacional implementado representa uma outra diferença em relação ao utilizado por BONNET e seus colaboradores ${ }^{[9], ~[46]}$, mas deve ser visto como uma extensão do proposto por VENTURINI ${ }^{[58]}$. As tensões e deformações equivalentes são acumuladas a cada iteração. Segue um resumo desse algorítmo:

(i) Cálculo das tensões elásticas $\left[N_{n}\right]$ para cada incremento $\mathrm{n}$ em ( $\mathbf{6 . 6}$ ), calculadas a partir da solução elástica $[N]$ da equação ( 5.6 ).

Solução iterativa: $\quad i=0$

(ii) $i=i+1$. Cálculo da tensão inicial a ser introduzida, através da equação ( 6.28 ), Seguida de ( 6.29 ). Na primeira iteração de cada incremento, o resíduo $Y$ é o incremento elástico dado por $\left[N_{n+1}\right]$.

(iii) Classificação dos pontos elásticos e plásticos: $\quad f \leq 0$ ?

(iv) Cálculo do multiplicador plástico $\Delta \lambda_{n+1}^{i}$, equação ( 6.18 ), para os pontos em regime plástico. 
(v) Atualização da tensão $\Delta \sigma_{n}^{i}=\mathbf{C}\left(\Delta \varepsilon^{i}{ }_{n}-\Delta \varepsilon^{p}{ }_{n}{ }^{i}\right)$ (equação ( 3.7$)$ ), dado que $\Delta \varepsilon_{n}^{\mathbf{p}^{i}}=\frac{\partial f}{\partial \sigma} \Delta \lambda \mathbf{n}$ (equação ( 3.10$)$ ).

(vi) Atualização da deformação equivalente acumulada, $\Delta \varepsilon_{\mathbf{n}}^{\mathbf{e q}^{i}}=\sqrt{\frac{2}{3}} \Delta \lambda_{n}{ }^{i}$.

(vii) Cálculo do resíduo, $Y\left(\Delta \boldsymbol{\varepsilon}_{n}^{i+1}\right)=\Delta \boldsymbol{\varepsilon}_{n}^{\mathbf{p}^{i}}$.

(viii) Cálculo do operador tangente consistente local, $\mathbf{C}_{n+1}^{\mathbf{e q}}{ }^{i}$, expressão ( $\mathbf{6 . 2 3}$ ). Montagem do operador tangente global.

(ix) Verificação da convergência, $\left[Y\left(\Delta \varepsilon_{n}^{i+1}\right)\right]<$ tolerância. Em caso de convergência, o resíduo $Y$ será adicionado ao novo incremento elástico $(n+2)$.

(x) Retorno a (ii), até completar-se o processo.

\subsection{Operadores tangentes para a plasticidade com gradiente}

Os resultados contidos no capítulo seguinte mostram que a formulação implícita do tipo Newton para o método dos elementos de contorno apresenta respostas bastante mais satisfatórias que a formulação convencional, em problemas mais exigentes quanto à precisão, como é o caso da localização de deformações, na qual o comportamento do campo de deformações sofre alterações abruptas.

O melhor desempenho da formulação implícita sugere que esta também deve ser considerada no caso da plasticidade com gradiente. No entanto, a formulação implícita anterior, válida para a plasticidade clássica, não pode ser empregada no caso da plasticidade com gradiente devido à elevação do grau da equação do multiplicador plástico decorrente da presença do termo do gradiente se segunda ordem.

A seguir duas possibilidades para a formulação implícita do método dos elementos de contorno são apresentadas para o caso da plasticidade com o segundo gradiente do multiplicador plástico. São novamente admitidos, sem restrição à generalidade, encruamento isótropo linear e critério de von Mises. 


\subsubsection{Operador tangente consistente para a plasticidade com gradiente}

Um primeiro procedimento implícito discutido aqui considera o operador tangente consistente (algorítmico) para correção da rigidez. São novamente admitidos, sem restrição à generalidade, encruamento isótropo linear e critério de von Mises. O desenvolvimento segue aquele utilizado no caso da plasticidade clássica. Mas neste caso, o critério de plastificação exprime-se como

$$
f_{n+1}=\sqrt{\frac{3}{2}}\left\|\mathbf{S}_{n+1}\right\|-\sigma_{0}-h \lambda_{n+1}+\omega\left(\nabla^{2} \lambda\right)_{n+1} \leq 0,
$$

sendo $\omega$ um parâmetro do material. A expressão anterior também pode ser escrita como

$$
f_{n+1}=\sqrt{\frac{3}{2}}\left\|\mathbf{S}_{n+1}\right\|-\sigma_{0}-h \lambda_{n}-h \Delta \lambda_{n}+\omega\left(\nabla^{2} \lambda\right)_{n}+\omega \Delta\left(\nabla^{2} \lambda\right)_{n} \leq 0 .
$$

( 6.31$)$

Como no caso da plasticidade local, numa etapa de previsão é utilizado um estado de tentativa elástico, a partir do qual é obtido o estado de tensão correto. Repetindo a relação obtida anteriormente entre esses dois estados, tem-se

$$
\mathbf{S}_{n+1}=\mathbf{S}_{n+1}{ }^{t}-2 \mu \Delta \lambda_{n} \mathbf{n}_{n+1},
$$

observando-se novamente que $\mathbf{S}_{n=1}$ e $\mathbf{S}_{n=1}^{t}$, estão alinhados com $\mathbf{n}_{n+1}$ (direção $\left.\mathbf{n}_{n+1} /\left\|\mathbf{n}_{n+1}\right\|\right), f_{n+1}$ pode ser reescrito como 


$$
\begin{gathered}
f_{n+1}=\sqrt{\frac{3}{2}}\left(\mathbf{S}_{n+1}{ }^{t}-2 \mu \Delta \lambda_{n} \mathbf{n}_{n+1}\right) \frac{\mathbf{n}_{n+1}}{\left\|\mathbf{n}_{n+1}\right\|}-\sigma_{0}-h \lambda_{n}-h \Delta \lambda_{n} \\
+\omega\left(\nabla^{2} \lambda\right)_{n}+\omega \Delta\left(\nabla^{2} \lambda\right)_{n} \leq 0,
\end{gathered}
$$

que possibilita a obtenção do multiplicador plástico através do método dos elementos de contorno, resolvendo-se o problema de potencial acima, ou

$$
\left(\sqrt{\frac{3}{2}}\left\|\mathbf{S}_{n+1}{ }^{t}\right\|-\sigma_{0}-h \lambda_{n}+\omega\left(\nabla^{2} \lambda\right)_{n}\right)-(3 \mu+h) \Delta \lambda_{n}+\omega \Delta\left(\nabla^{2} \lambda\right)_{n}=0
$$

para pontos em regime plástico.

Uma vez determinado $\Delta \lambda_{n}$ pelo método dos elementos de contorno, conforme indicado acima, é possível retornar à equação anterior, a fim de se determinar o gradiente plástico:

$$
\omega \Delta\left(\nabla^{2} \lambda\right)_{n}=(3 \mu+h) \Delta \lambda_{n}-\left(\sqrt{\frac{3}{2}} \mid \mathbf{S}_{n+1}{ }^{t} \|-\sigma_{0}-h \lambda_{n}+\omega\left(\nabla^{2} \lambda\right)_{n}\right)
$$

que é necessário no problema do gradiente e deve ser utilizado também na determinação do operador tangente local $\mathbf{C}_{n+1}^{\mathbf{e p}}$, o que é feito a seguir.

Da condição de consistência $(\dot{f}=0)$ obtida acima,

$$
\Delta \lambda_{n}=\frac{\left(\sqrt{\frac{3}{2}}\left\|\mathbf{S}_{n+1}{ }^{t}\right\|-\sigma_{0}-h \lambda_{n}+\omega\left(\nabla^{2} \lambda\right)_{n}\right)+\omega \Delta\left(\nabla^{2} \lambda\right)_{n}}{(3 \mu+h)} .
$$

Do tensor de Cauchy e da expressão para a deformação plástica, tem-se 


$$
\Delta \sigma=\mathbf{C} \Delta \varepsilon-\mathbf{C} \frac{\partial f}{\partial \sigma} \Delta \lambda
$$

Para se obter o operador tangente algorítmico, deriva-se a expressão acima em relação à deformação no "tempo" $t_{n+1}, \varepsilon_{n+1}^{i}$.Lembrando-se a notação

$$
\mathbf{n}=\frac{\partial f}{\partial \sigma}
$$

resulta

$$
\mathbf{C}_{n+1}^{\mathbf{e p}}=\frac{\partial \boldsymbol{\sigma}_{n+1}^{i}}{\partial \boldsymbol{\varepsilon}_{n+1}^{i}}=\left[\mathbf{C}-\frac{\partial}{\partial \Delta \boldsymbol{\varepsilon}_{n}^{i}}\left(\mathbf{C n}_{n+1}^{i} \Delta \lambda_{n}^{i}\right)\right],
$$

que define o operador tangente algorítmo como no caso da plasticidade clássica, ( 6.20 ) - ( 6.22 ), expressando a relação entre tensão e deformação total. A diferença é que neste caso o incremento do multiplicador plástico depende também do termo do gradiente. No entanto, para o termo do gradiente, que exprime apenas uma correlação espacial definindo o comprimento característico, pode-se escrever

$$
\frac{\partial\left(\nabla^{2} \lambda\right)}{\partial \varepsilon}=0
$$

Como os outros termos todos já apareciam no operador tangente algorítmico para a plasticidade local, a expressão obtida naquele caso é a mesma que representa esse operador para a plasticidade com gradiente: 


$$
\begin{gathered}
\mathbf{C}_{n+1}^{e p}=\frac{\partial \sigma_{n+1}}{\partial \Delta \varepsilon_{n}}=\mathbf{C}-2 \mu \theta_{n+1}\left(\mathbf{I}-\frac{1}{3} \mathbf{I} \otimes \mathbf{I}\right)-2 \mu \overline{\theta_{n+1}}\left(\hat{\mathbf{n}}_{n+1} \otimes \hat{\mathbf{n}}_{n+1}\right) \\
\operatorname{com} \quad \theta_{n+1}=2 \mu \frac{\Delta \lambda_{n}}{\left\|\mathbf{S}_{n+1}\right\|} \\
e \quad \overline{\theta_{n+1}}=\left(\frac{1}{1+\frac{h}{3 \mu}}\right)-\theta_{n+1} .
\end{gathered}
$$

De um modo mais geral, a derivada de $\Delta \lambda$ pode ser determinada numericamente, como indicado a seguir. Para uma exposição geral mais completa e detalhada, ver FUDOLI, VENTURINI e BENALLAL ${ }^{[29]}$.

A representação algébrica da equação ( $\mathbf{5 . 1 0}$ ) para $\Delta \lambda$ pode ser reagrupada como

$$
\{\Delta \lambda\}_{x}=[H]_{x}^{-1}\left(\{F\}_{x}+[V]\{B(\Delta \boldsymbol{\varepsilon})\}\right)
$$

onde $\{\Delta \lambda\}_{x}$ representa o vetor de incógnitas, $\{F\}_{x}$ corresponde ao vetor que resulta dos valores prescritos e $[H]_{x}^{-1}$ à matriz de coeficientes das incógnitas.

A derivada de $\Delta \lambda$ num ponto $k$, resulta então em

$$
\frac{\partial \Delta \lambda_{k}}{\partial \Delta \varepsilon_{k}}=\{H\}_{x}^{-1}\{V\}_{k} \frac{\partial B\left(\Delta \varepsilon_{k}\right)}{\partial \Delta \varepsilon_{k}}
$$

$\operatorname{com}\{H\}_{x}^{-1}$ correspondendo à k-ésima linha de $[H]_{x}^{-1}$ e $\{V\}_{k}$ à k-ésima coluna de $[V]$.

Observe-se que os termos provenientes do laplaciano não contribui para a derivada de $\Delta \lambda$. 


\subsubsection{Operador tangente contínuo para a plasticidade com gradiente}

A seguir, uma segunda formulação implícita do método dos elementos de contorno é derivada, para efeito de comparação com o operador tangente consistente. Neste caso é considerado o operador tangente contínuo, estendido para o caso da plasticidade com o segundo gradiente do multiplicador plástico.

Recorde-se que o problema é resolvido pela equação ( 5.6 ), ou

$$
[\mathbf{C \varepsilon}]=[N]+[\bar{S}]\left[\boldsymbol{\sigma}^{p}\right],
$$

onde

$$
\sigma^{p}=\mathbf{C} \varepsilon-\sigma=\mathbf{C} \varepsilon-\mathbf{C}_{n+1}^{\mathrm{ep}} \boldsymbol{\varepsilon}
$$

Para obter o operador tangente contínuo, considere-se a condição de consistência:

$$
\mathbf{n C} \dot{\varepsilon}-H \dot{\lambda}+\omega \Delta\left(\nabla^{2} \dot{\lambda}\right)=0 .
$$

Substituindo-se o valor do multiplicador plástico da equação anterior no tensor de Cauchy:

$$
\dot{\sigma}=\mathbf{C}(\dot{\varepsilon}-\mathbf{n} \dot{\lambda})=\mathbf{C}\left(\dot{\varepsilon}-\mathbf{n} \frac{\mathbf{n C} \dot{\varepsilon}+\omega \Delta\left(\nabla^{2} \dot{\lambda}\right)}{H}\right)
$$


Derivando-se em relação à deformação total e considerando-se novamente que o termo do gradiente não contribui, resulta o mesmo que na plasticidade clássica:

$$
\mathbf{C}_{n+1}^{\mathbf{e p}}=\mathbf{C}-\frac{\mathbf{C n} \otimes \mathbf{n C}}{H},
$$

( 6.48$)$

o que permite que se escreva

$$
\sigma^{p}=\frac{\mathbf{C n} \otimes \mathbf{n C}}{H} \varepsilon, \quad H=h+\mathbf{n C n},
$$

( 6.49 )

ou ainda

$$
\sigma^{p}=\mathbf{D} \boldsymbol{\varepsilon}=\frac{\mathbf{C n} \otimes \mathbf{n}}{H} \mathbf{C} \boldsymbol{\varepsilon}, \quad H=h+\mathbf{n C n}
$$

( 6.50$)$

Num passo $n+1$, pode-se então escrever

$$
\left[\mathbf{C} \boldsymbol{\varepsilon}_{n}\right]+\left[\mathbf{C} \Delta \boldsymbol{\varepsilon}_{n}\right]=[N]+[\bar{S}]\left(\left[\boldsymbol{\sigma}_{n}{ }^{p}\right]+\left[\mathbf{D}_{n+1}\right]\left[\mathbf{C} \Delta \boldsymbol{\varepsilon}_{n}\right]\right)
$$

ou

$$
\left[S^{e p}\right]\left[\mathbf{C} \Delta \boldsymbol{\varepsilon}_{n}\right]=\left([\mathbf{I}]-[\bar{S}]\left[\mathbf{D}_{n+1}\right]\right)\left[\mathbf{C} \Delta \boldsymbol{\varepsilon}_{n}\right]=[N]+[\bar{S}]\left[\sigma_{n}{ }^{p}\right]-\left[\mathbf{C} \boldsymbol{\varepsilon}_{n}\right]
$$

com $\left[S^{e p}\right]$ representando a matriz de rigidez tangente global. 


\subsection{Uma formulação alternativa para o método dos elementos de contorno}

Esta formulação não chegou a ser implementada no código computacional, mas fica sugerida aqui. Pode constituir uma alternativa em algum caso, como talvez na utilização de leis constitutivas mais complicadas que as detalhadas neste trabalho.

A alternativa consiste em se utilizar a equação do multiplicador plástico acoplada com a seguinte, no lugar da equação ( 6.9 ):

$$
\begin{aligned}
{\left[\bar{Y}\left(\Delta \varepsilon_{n}^{i}, \Delta \lambda_{n}^{i}\right)\right] } & =[C]\left[\delta \Delta \varepsilon_{n}^{i}\right] \\
& -[\bar{S}]\left[\left([C]-\mathbf{C}^{\mathrm{epC} E L}\right)\left[\delta \Delta \varepsilon_{n}^{i}\right]+\mathbf{C}^{\mathrm{epC} E L} \mathbf{n} \delta \Delta \lambda_{n}^{i}\right]
\end{aligned}
$$

onde

$$
\mathbf{C}^{\mathrm{epC} E L}=\frac{\partial \boldsymbol{\sigma}_{n}^{\mathrm{arr} i}}{\partial \Delta \varepsilon_{E L}^{i}{ }_{n}}
$$

representa um operador tangente elástico consistente.

Neste caso, portanto, a equação ( 6.53 ) é que fica acoplada à do multiplicador plástico, que por sua vez é reescrita abaixo como

$$
\mathbf{n C} \Delta \varepsilon-H \Delta \lambda-w \Delta\left(\nabla^{2} \lambda\right)=0 .
$$

A relação ( 6.53 ) é obtida de ( 6.9 ) a partir das expressões 


$$
\begin{gathered}
\frac{\partial \sigma}{\partial \Delta \varepsilon} \delta \varepsilon=\Delta \sigma=\frac{\partial \sigma}{\partial \Delta \varepsilon_{E L}} \delta \varepsilon_{E L} \\
e \\
\delta \varepsilon_{E L}=\delta \varepsilon-\delta \lambda \mathbf{n} .
\end{gathered}
$$

( 6.56$)$

O operador tangente elástico consistente pode ser obtido da relação

$$
\Delta \sigma=\mathbf{C}(\Delta \varepsilon-\mathbf{n} \Delta \lambda)
$$

que deve ser derivada em relação ao tempo (simbolizada abaixo por ' $d$ '), resultando, após um reagrupamento dos termos,

$$
d \sigma=\left(\mathbf{C}-\frac{\partial \mathbf{n}}{\partial \sigma}\right)^{-1}(d \varepsilon-\mathbf{n} d \lambda) .
$$

Lembrando-se que $\mathbf{n}=(\partial f / \partial \sigma)$, tem-se

$$
\mathbf{C}^{\mathrm{epC} E L}=\frac{\partial \sigma^{\mathrm{arr}}}{\partial \Delta \varepsilon_{E L}}=\left(\mathbf{C}-\frac{\partial^{2} f}{\partial \sigma^{2}}\right)^{-1}
$$

Observe-se que a expressão ( 6.53 ) reconduz ao procedimento explícito convencional se a matriz tangente nunca for atualizada, ou seja,

$$
\mathbf{C}^{\mathrm{epC} E L}=\mathbf{C} .
$$




\section{Exemplos}

São apresentadas três séries de exemplos. Geometria e condições de contorno são as mesmas em todas elas. Os dados comuns são apresentados na Figura 7.1. Tratase de um retângulo sobre compressão, imposta pelo deslocamento $\delta$ prescrito na extremidade. $\mathrm{O}$ valor de $\delta$ em cada simulação pode ser observado na curva reaçãodeslocamento, mas em nenhum caso é maior que $0,024 \mathrm{~mm}$. Nos casos em que $\delta$ é menor que 0,024 significa que houve perda de estabilidade numérica antes desse valor. O procedimento implícito tem convergência superior ao do explícito local, mas às vezes o valor de $\delta$ é limitado ao determinado na simulação pelo método explícito local, para efeito de comparação. O procedimento explícito com o gradiente também é mais estável que o local, graças justamente ao efeito estabilizador do gradiente.

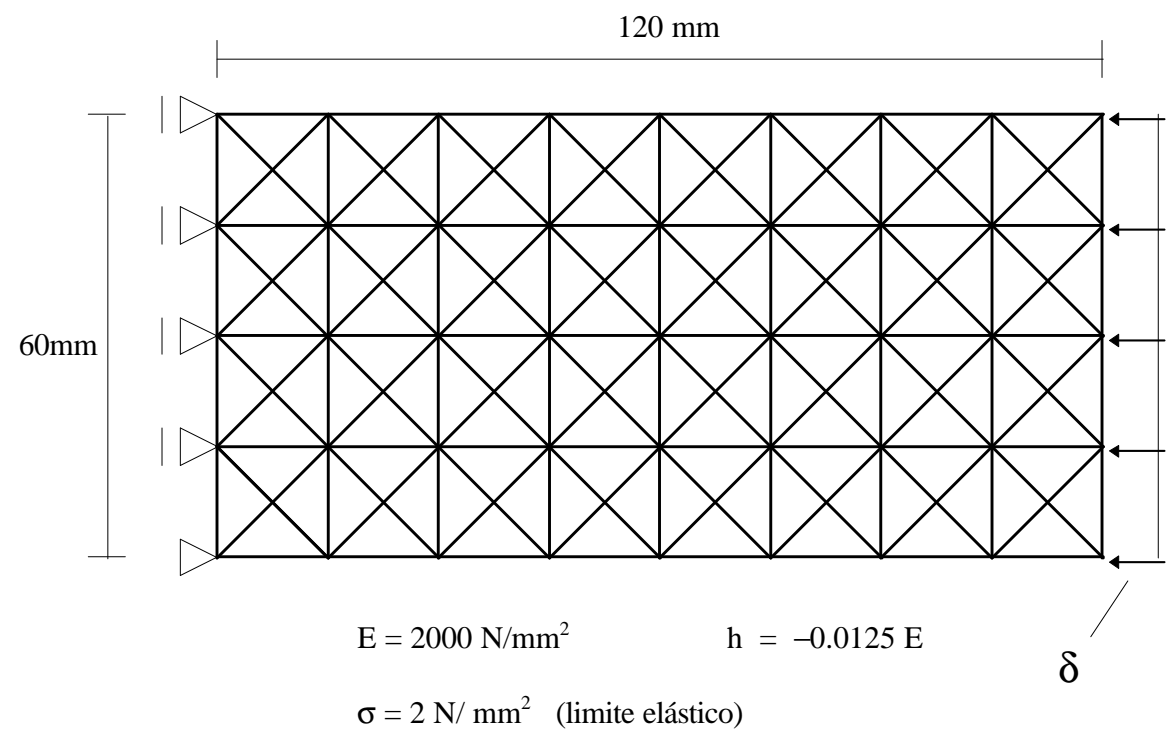

Figura 7.1 -- Dados para o problema do retângulo sob compressão. 
Os outros dados que faltam aparecem abaixo das figuras. $v$ representa $o$ coeficiente de Poisson. $\omega$ é o coeficiente do termo do segundo gradiente (um parâmetro do material).

As configurações mostradas nas figuras dos deslocamentos plásticos são as deformadas. São considerados exclusivamente os deslocamentos plásticos (elásticos eliminados).

As cores nas configurações deformadas referem-se à tensão normal na direção horizontal.

\subsection{Primeira série: retângulo com enfraquecimento no canto}

O menor quadrado no canto inferior tem seu limite elástico enfraquecido em 10\%. (Nas malhas mais refinadas, ainda toma-se o menor retângulo. De modo que a área enfraquecida diminui.)

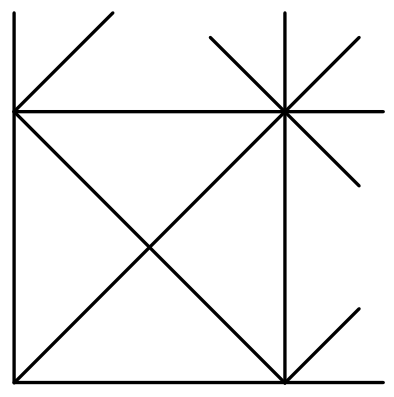

Figura 7.2 - Quadrado enfraquecido no canto inferior esquerdo do retângulo.

O enfraquecimento pelo limite de tensão é uma maneira de precipitar a localização de deformações de forma mais controlada. Mas na presença de amolecimento por deformação, pode ocorrer localização apenas devido a diferenças produzidas pela aproximação, ou pela própria precisão numérica. 


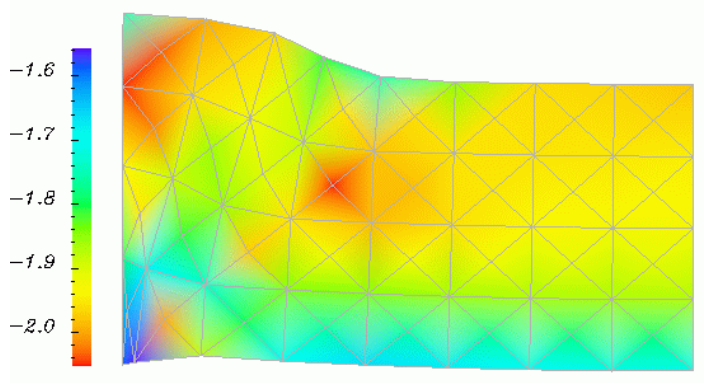

$\sigma_{x x}$

Deslocamento Plastico (X1300)

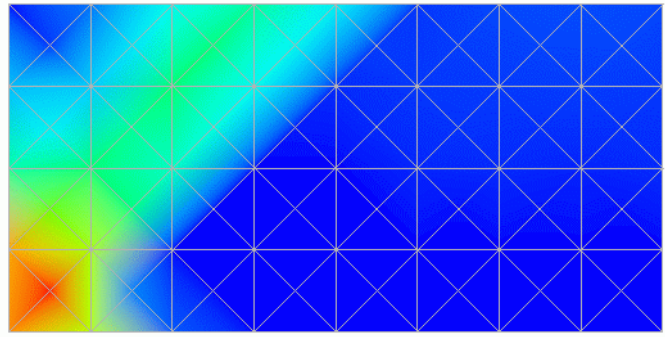

$$
\begin{array}{lllll}
8 & 0 & 4 & 0 & 8 \\
8 & 8 & 8 & 8 & 8 \\
0 & 0 & 0 & 0 & 8 \\
0 & 0 & 0 & 0 & 0
\end{array}
$$

Deformacao equivalente acumulada
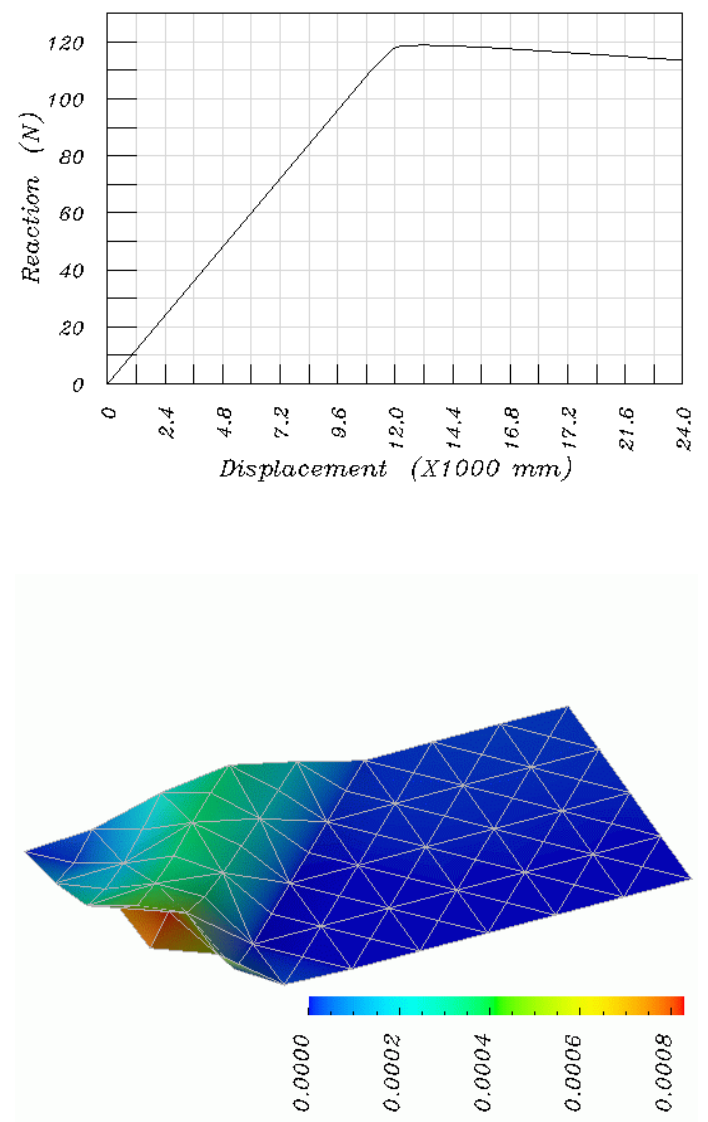

Deformacao equivalente acumulada

Figura 7.3 - Série 1: 128 células. Procedimento explícito. Plasticidade local.

$\delta=0,024 \mathrm{~mm}, v=0,0.400$ incrementos de carga.

Neste caso é possível notar uma discreta manifestação da localização, conforme evidencia a figura seguinte, utilizando plasticidade com gradiente. Devido à malha graúda, o efeito da localização é pequeno, pois a faixa de concentração de deformações acompanha o tamanho da malha. 

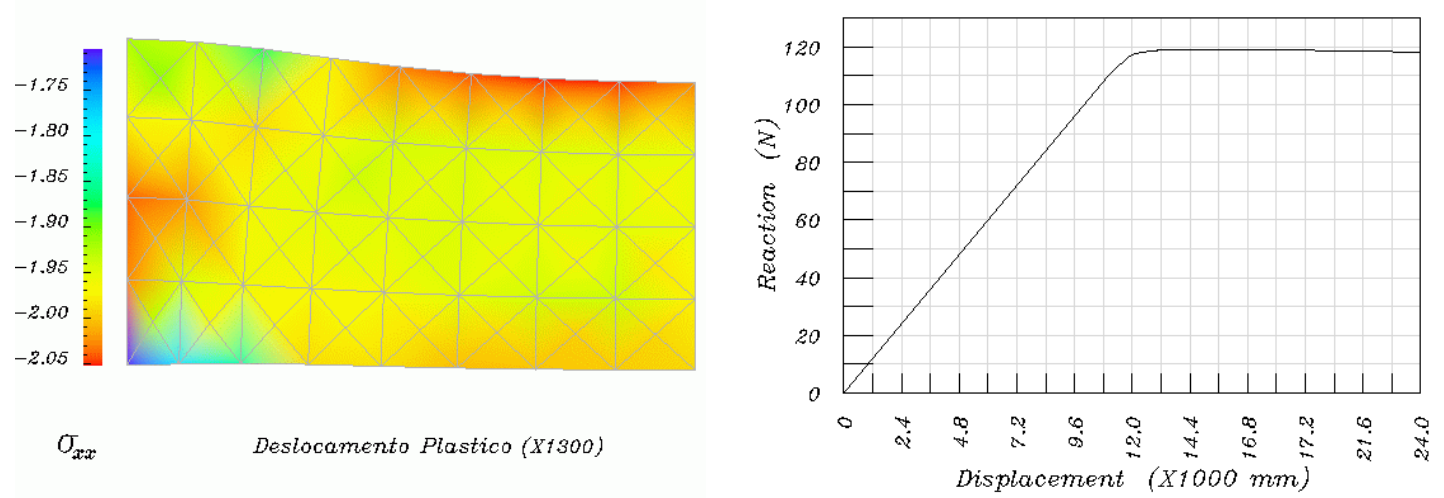

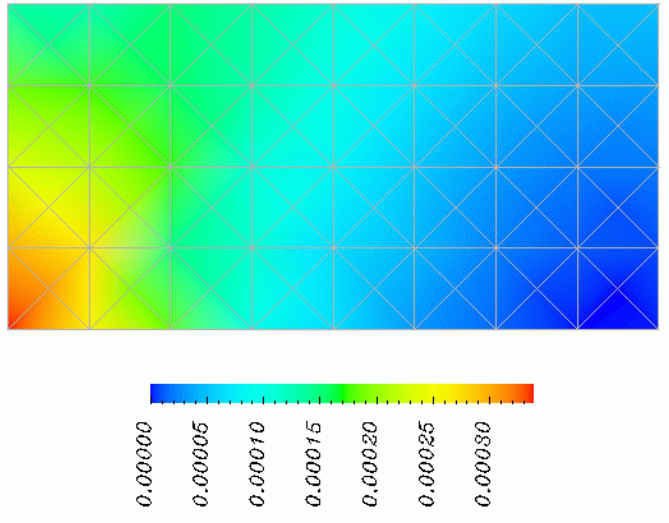

Deformacao equivalente acumulada

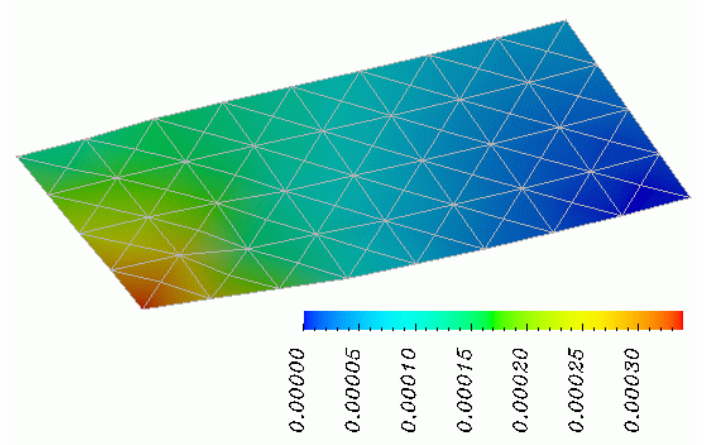

Deformacao equivalente acumulada

Figura 7.4 - Série 1: 128 células. Procedimento explícito. Plasticidade com gradiente. $\delta=0,024 \mathrm{~mm}, v=0,0 . \omega=500000 \mathrm{~N} .400$ incrementos de carga.

A comparação com a figura anterior mostra que o (discreto) efeito da localização detectado para a plasticidade local é anulado na formulação com gradiente (dependendo do valor de $\omega$ ). A diminuição da reação é neste caso governada pelo encruamento negativo, e não pela localização, como na simulação anterior. A deformação equivalente também é nitidamente diferente do anterior, e mesmo a configuração deformada apresenta alterações perceptíveis. 

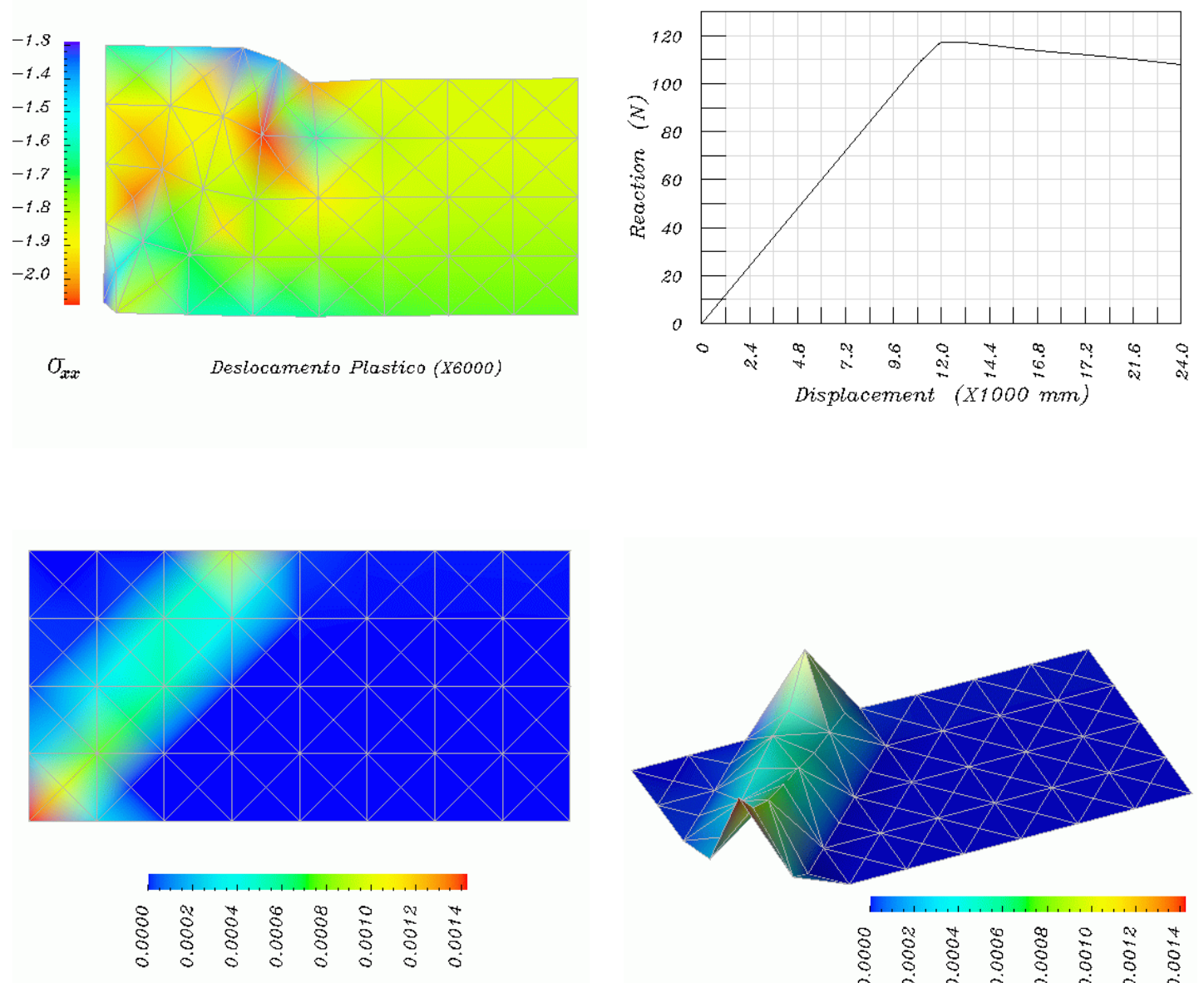

Deformacao equivalente acumulada

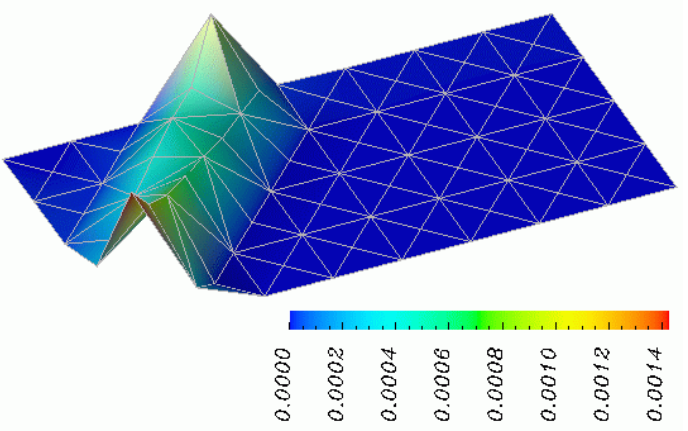

Deformacao equivalente acumulada

Figura 7.5 - Série 1: 128 células. Procedimento implícito. Plasticidade local. $\delta=0,024 \mathrm{~mm}, v=0,0.400$ incrementos de carga.

No caso da simulação utilizando-se a formulação implícita, o efeito da localização se mostra muito mais acentuado. Todas imagens dessa figura mostram que o método dos elementos de contorno consegue neste caso capturar o fenômeno da localização de modo bastante claro e qualificado. 

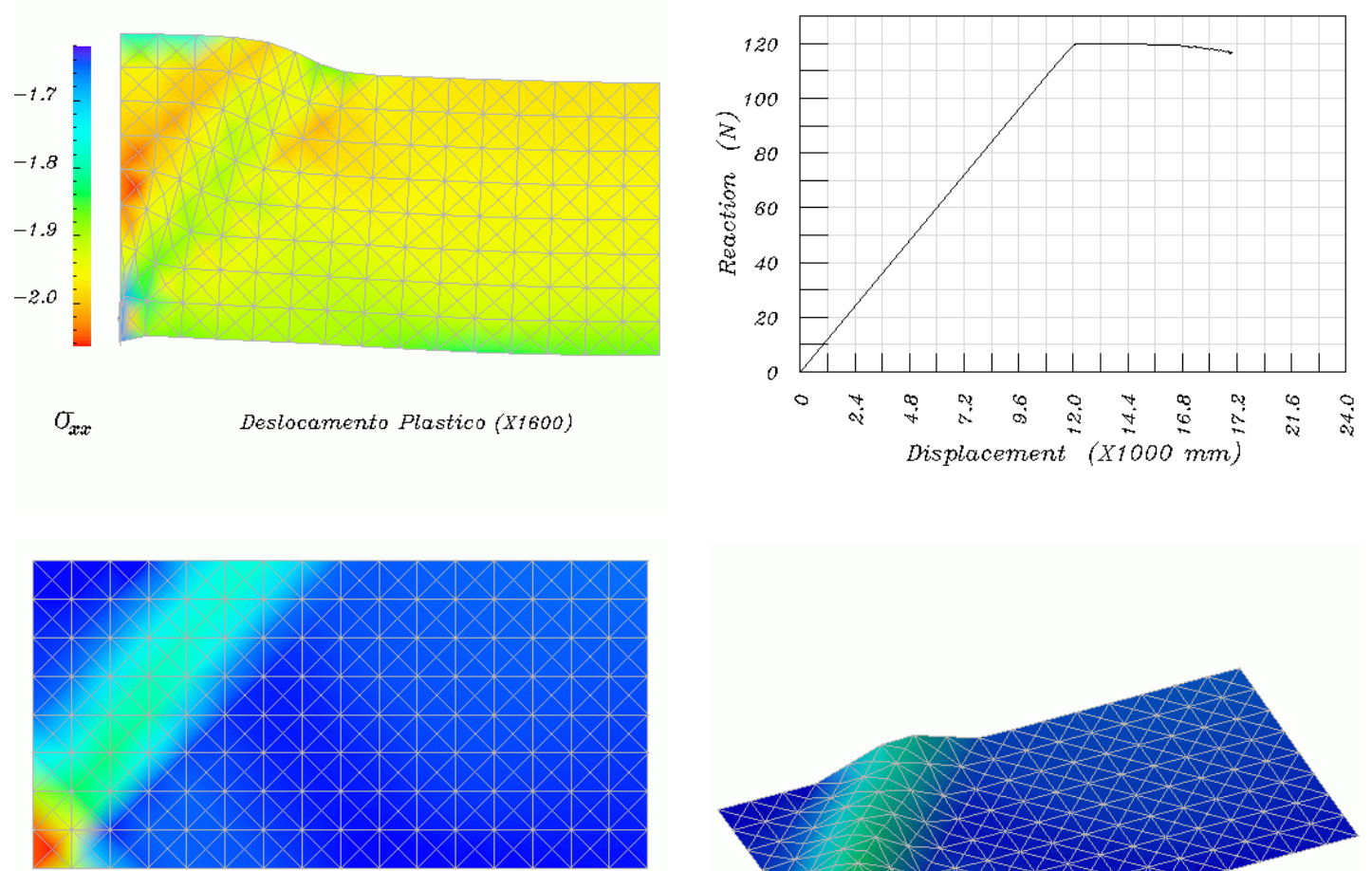

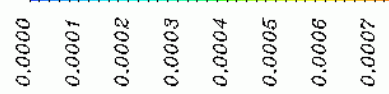

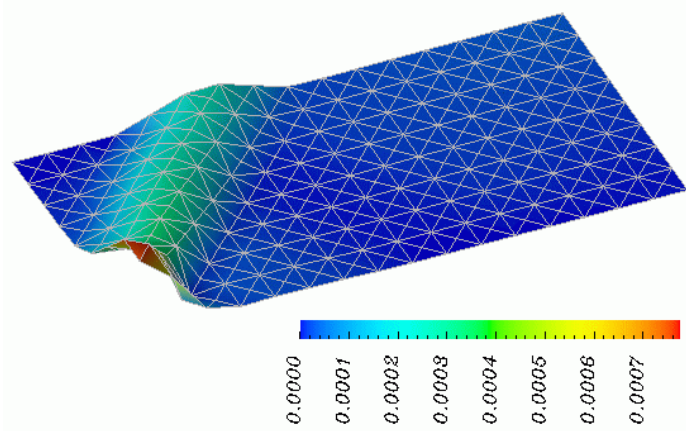

Deformacao equivalente acumulada

Deformacao equivalente acumulada

Figura 7.6 - Série 1: 512 células. Procedimento explícito. Plasticidade local.

$\delta=0,019 \mathrm{~mm}, v=0,0.400$ incrementos de carga.

Note-se que neste caso o deslocamento na extremidade chega apenas a 0,019 mm. A região de dissipação de energia diminui com o tamanho da malha, e através do procedimento explícito, ao iniciar-se o processo de localização, a solução instabiliza-se rapidamente. 

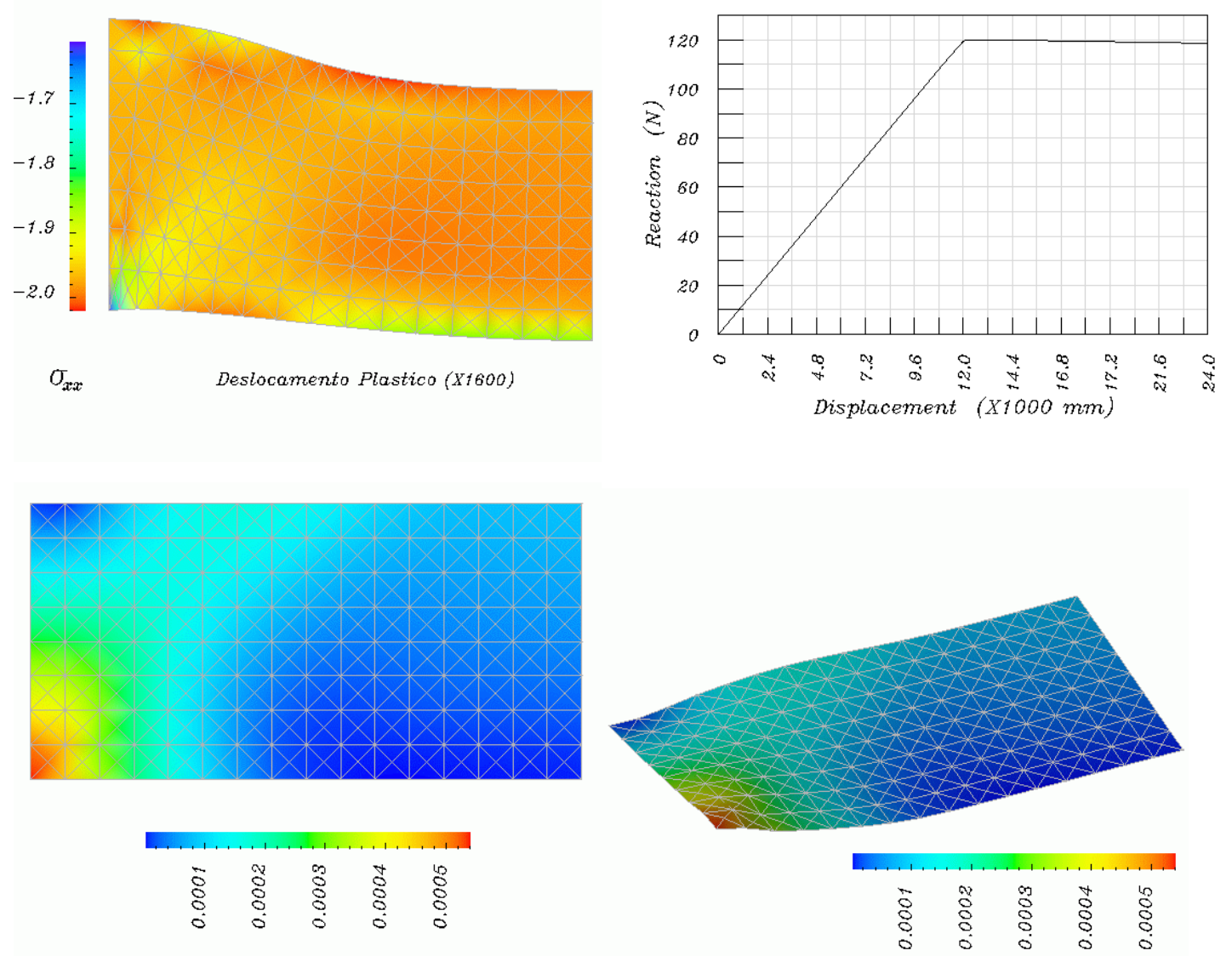

$$
\begin{array}{lllll}
5 & 0 & 0 & 1 & 8 \\
8 & 8 & 8 & 8 & 8 \\
0 & 0 & 0 & 8 & 8 \\
0 & 0 & 0 & 0
\end{array}
$$

Deformacao equivalente acumulada

Deformacao equivalente acumulada

Figura 7.7 - Série 1: 512 células. Procedimento explícito. Plasticidade com gradiente. $\delta=0,024 \mathrm{~mm}, v=0,0 . \omega=50000 \mathrm{~N} .400$ incrementos de carga.

Neste exemplo o efeito regularizador do gradiente é bem claro. A localização é bastante suavizada. A curva reação-deslocamento é bem próxima do que ocorreria num corpo homogêneo. A estabilidade do processo é mantida até o final, sem problemas. 

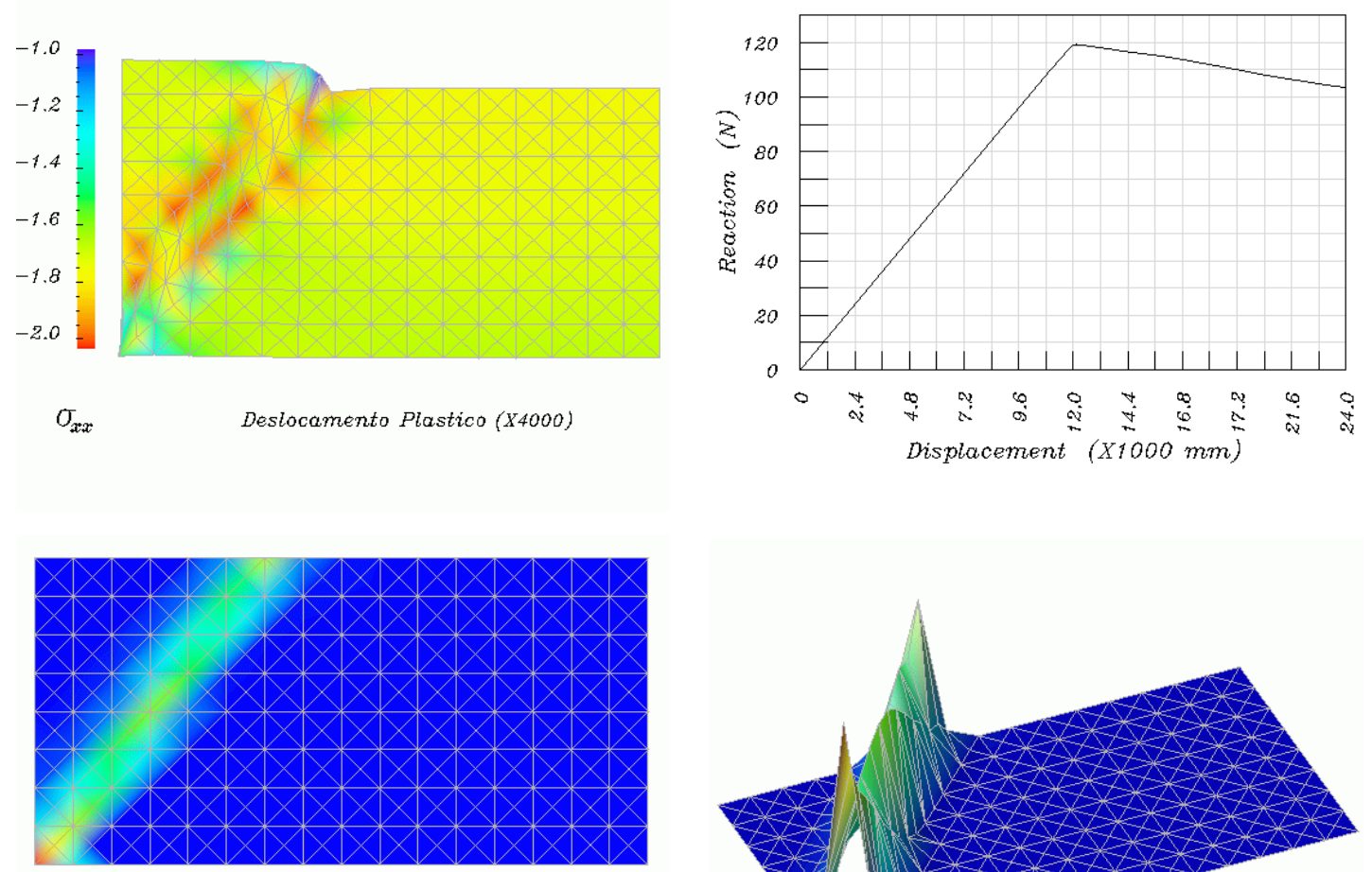

$\begin{array}{llllll}8 & 1 & 0 & 4 & 8 & 4 \\ 8 & 8 & 5 & 5 & 8 & 8 \\ 0 & 8 & 8 & 8 & 8 & 8 \\ 0 & 0 & 0 & 0 & 0 & 0\end{array}$

Deformacao equivalente acumulada

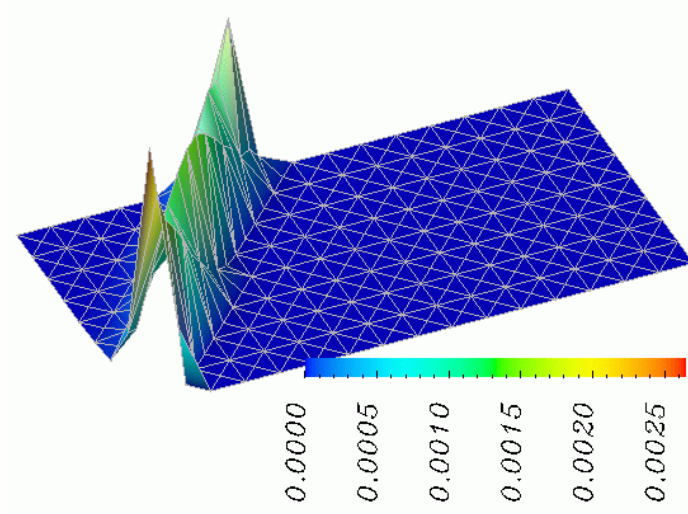

Deformacao equivalente acumulada

Figura 7.8 - Série 1: 512células. Procedimento implícito. Plasticidade local. $\delta=0,024 \mathrm{~mm}, v=0,0.400$ incrementos de carga.

A localização agora é ainda mais acentuada. Conforme esperado, a diminuição do tamanho da malha torna mais crítica a solução. Apesar disso, a solução pelo método implícito ainda é completamente estável. 

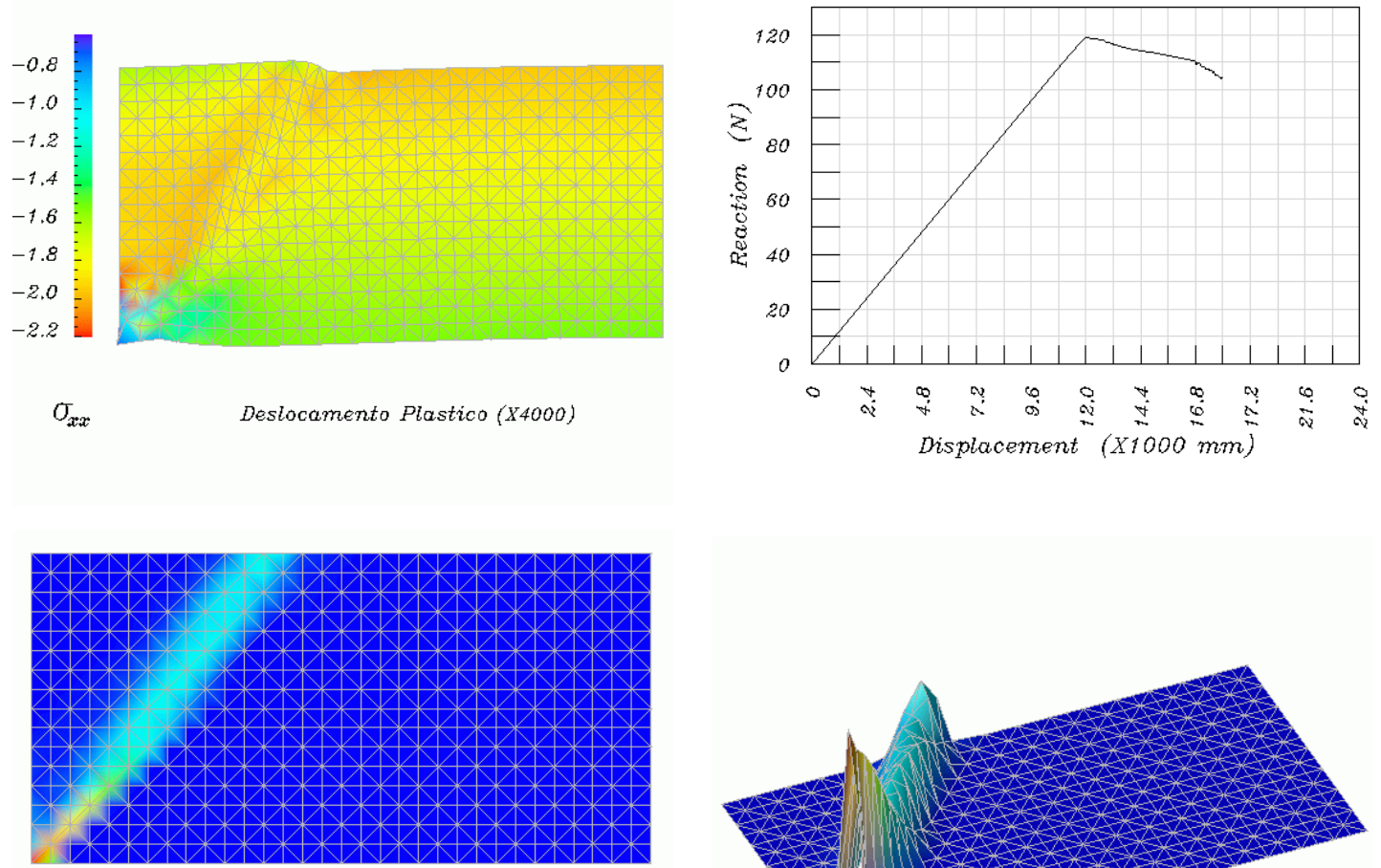

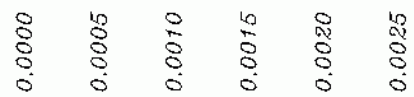

Deformacao equivalente acumulada

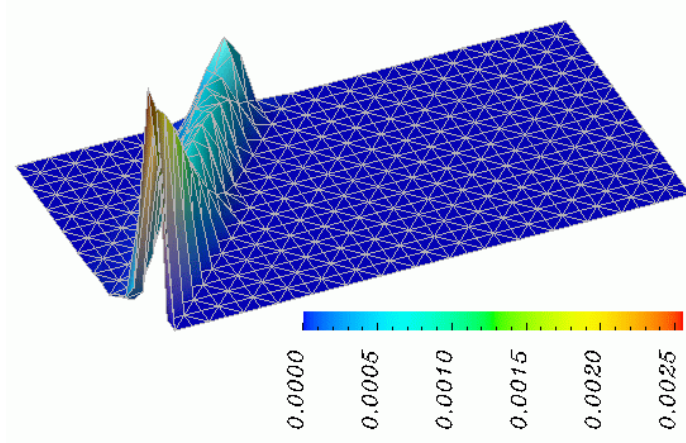

Deformacao equivalente acumulada

Figura 7.9 - Série 1: 1024 células. Procedimento implícito. Plasticidade local $\delta=0,024 \mathrm{~mm}, v=0,0.400$ incrementos de carga.

Neste exemplo foi enfraquecido mais um ponto, na direção da faixa de localização, na seqüência da diagonal do quadrado enfraquecido. Isto é indicado na imagem bidimensional da deformação equivalente, observando um valor alto naquele ponto.

A malha de 1024 células encontra-se numa posição desfavorável em relação à formação da faixa de localização na diagonal. Na verdade a largura da faixa de localização não coincide muito bem com a menor largura da malha, e tenta se situar em algum ponto entre a largura do menor quadrado e o dobro deste tamanho. O resultado é que, para este problema, a malha de 1024 células é sensivelmente menos estável que a de 512 células. Neste caso só foram obtidos resultados mais 
satisfatórios quando se enfraqueceu mais um ponto, para fora da diagonal do quadrado enfraquecido de canto, que corresponde à diagonal do quadrado de lado duas vezes maior que a largura da malha. A malha de 256 células também apresenta o mesmo tipo de comportamento.

Isto mostra que a largura efetiva da malha também depende de seu posicionamento em relação aos campos de deformação ou tensão.
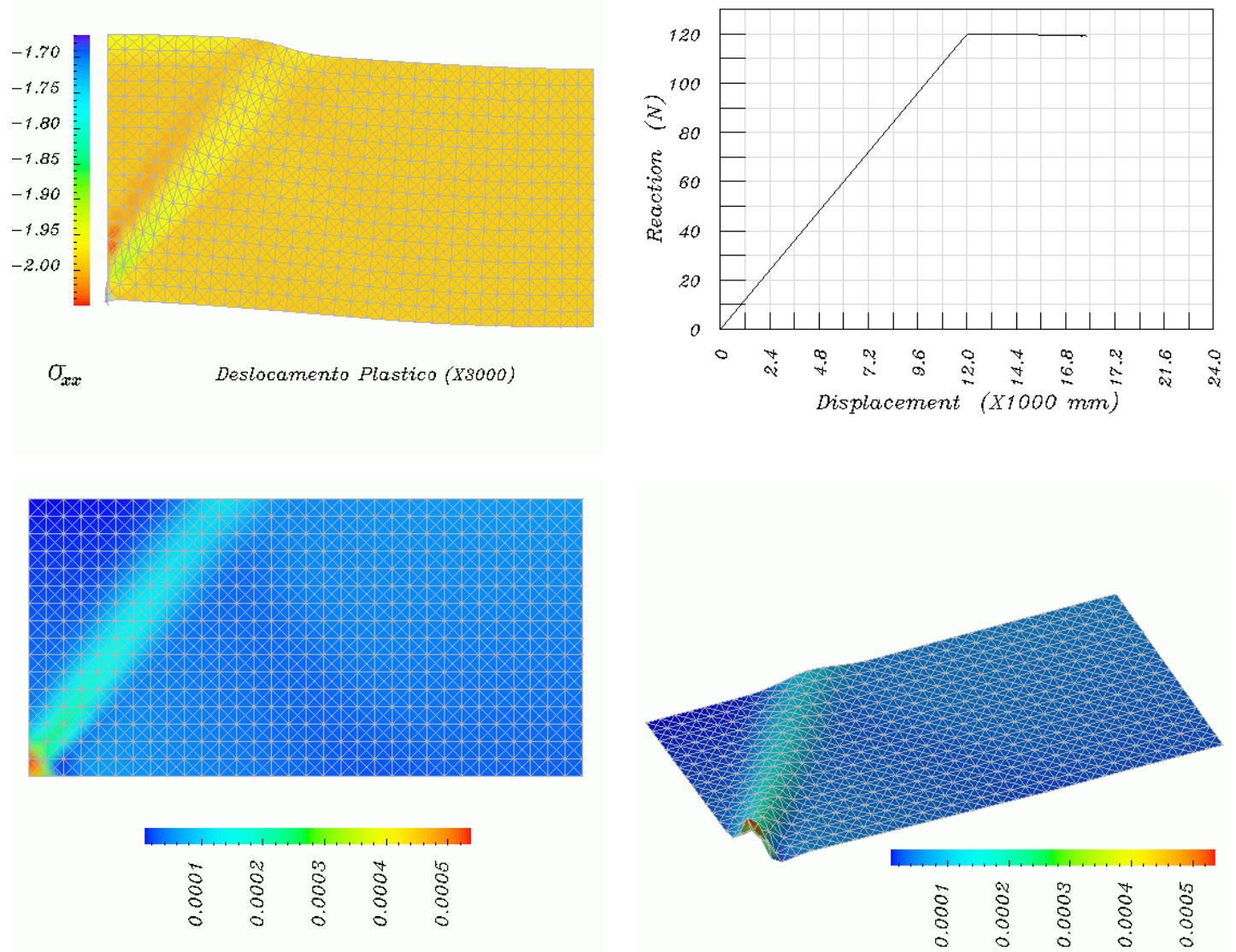

Deformacao equivalente acumulada

Deformacao equivalente acumulada

Figura 7.10 - Série 1: 2048 células. Procedimento explícito. Plasticidade local. $\delta=0,0178 \mathrm{~mm}, v=0,0.400$ incrementos de carga.

Nesta discretização mais refinada, o procedimento explícito não consegue mais capturar a localização, evidenciando uma limitação da convergência do método, 
nesta formulação. Além disso, o processo se instabiliza rapidamente, com as deformações se concentrando no canto enfraquecido. Como não se evidencia mais a localização com o modelo local, não faz mais muito sentido comparar o efeito do gradiente. As deformações já estão se espalhando numa faixa, com valores não muito altos (exceto no canto enfraquecido). Ainda assim, vale a pena olhar o resultado com gradiente abaixo. Embora o efeito neste caso seja muito pequeno, é possível ao menos verificar que o gradiente continua espalhando as deformações. A faixa de maior concentração de deformação é ligeiramente maior que no caso local.
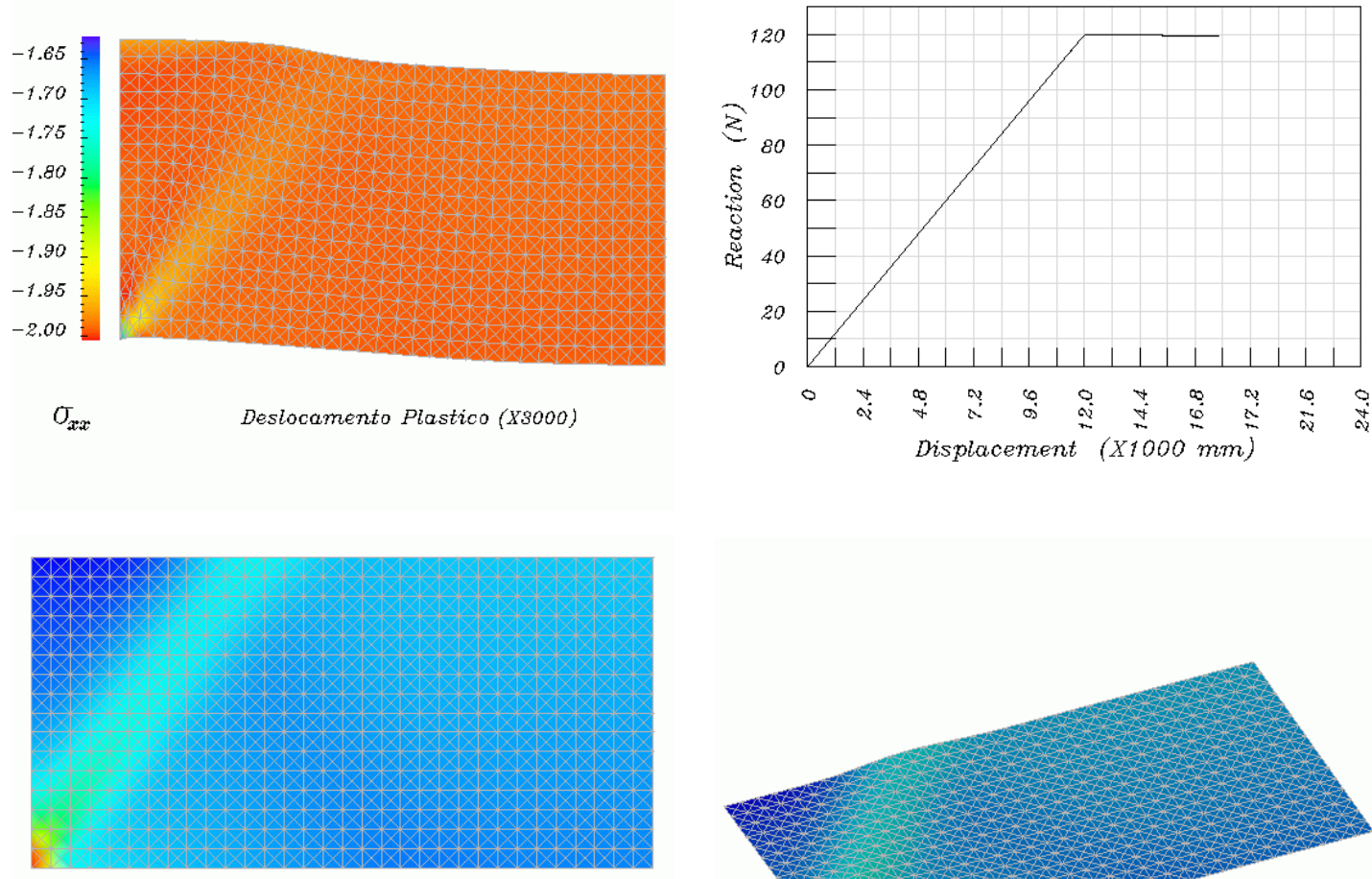

$$
\begin{array}{llllll}
8 & 0 & 0 & 9 & 0 & 0 \\
8 & 8 & 8 & 8 & 8 & 8 \\
8 & 8 & 8 & 8 & 8 & 8 \\
0 & 8 & 0 & 8 & 8 & 8 \\
0 & 0 & 0 & 0 & 0 & 0
\end{array}
$$

Deformacao equivalente acumulada

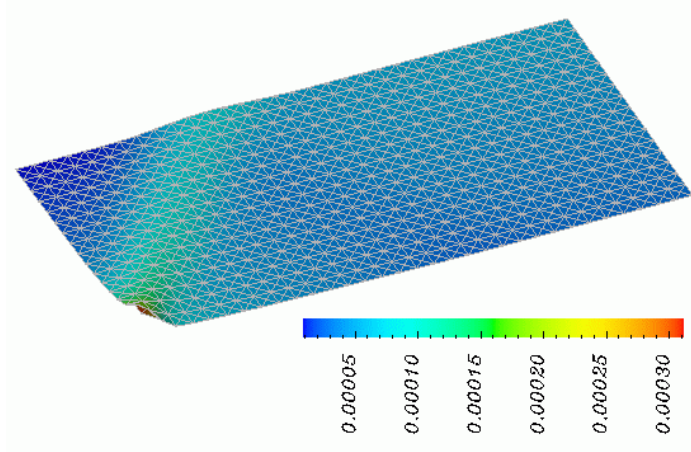

Deformacao equivalente acumulada

Figura 7.11 - Série 1: 2048 células. Procedimento explícito. Plasticidade com gradiente. $\delta=0,024 \mathrm{~mm}, v=0,0 . \omega=5000 \mathrm{~N}$. 400 incrementos de carga. 

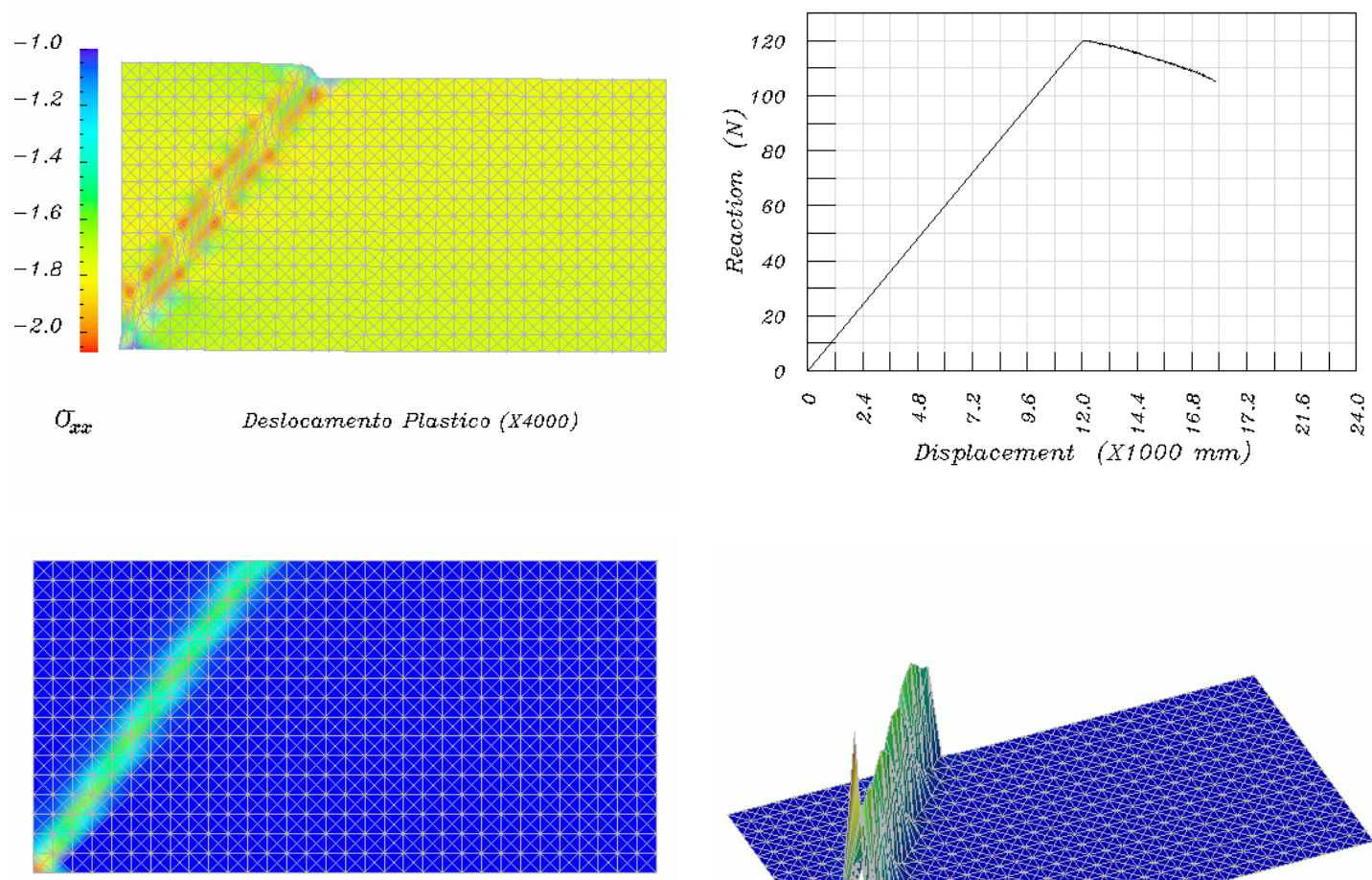

$$
\begin{array}{llllll}
8 & 8 & 0 & 0 & 8 & 1 \\
8 & 8 & 0 & 0 & 8 & 8 \\
8 & 0 & 0 & 8 & 8 & 8 \\
0 & 0 & 0 & 0 & 0 & 0
\end{array}
$$

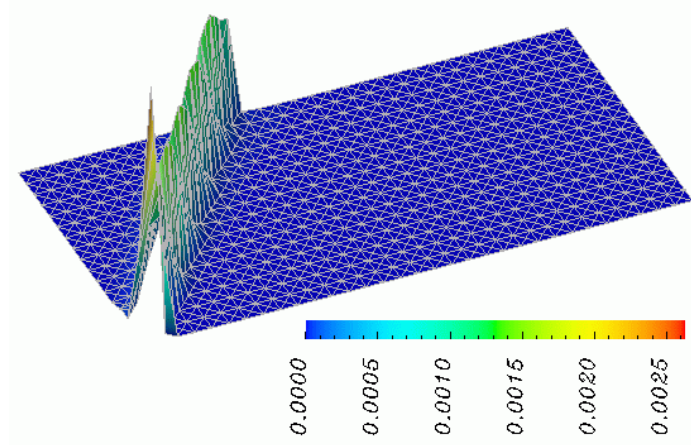

Deformacao equivalente acumulada

Figura 7.12 - Série 1: 2048 células. Procedimento implícito. Plasticidade local. $\delta=0,0178 \mathrm{~mm}, v=0,0.400$ incrementos de carga.

Nesta discretização, os resultados obtidos com o procedimento implícito comparados aos do explícito são impressionates. O procedimento também é interrompido antes da imposição de $\delta=0.024 \mathrm{~mm}$, o que é esperado, na atual implementação, pois a deformação concentra-se agora numa faixa bem menor para dar conta de toda dissipação.

É conveniente observar-se que o procedimento implícito requer um número menor de incrementos de carga. Aqui é utilizado o mesmo para facilitar a comparação. 


\subsection{Segunda série: enfraquecimento central}

O mesmo retângulo definido na Figura 7.1 é utilizado nesta segunda série. A diferença é que neste caso o enfraquecimento é imposto no centro do retângulo. Conforme mostram os exemplos, esta é uma situação mais estável numericamente, aparentemente porque as soluções podem se manifestar mais livremente, enquanto no exemplo de canto apenas uma podia se desenvolver. A figura a seguir assinala os pontos enfraquecidos, bem no centro do retângulo. São utilizados de um a três pontos enfraquecidos, nesta série. Nos exemplos utilizando método explícito são sempre tomados três pontos. Embora três pontos nessa posição possa significar (conforme a malha) duas vezes a largura da malha, ainda representa a diagonal do menor retângulo.

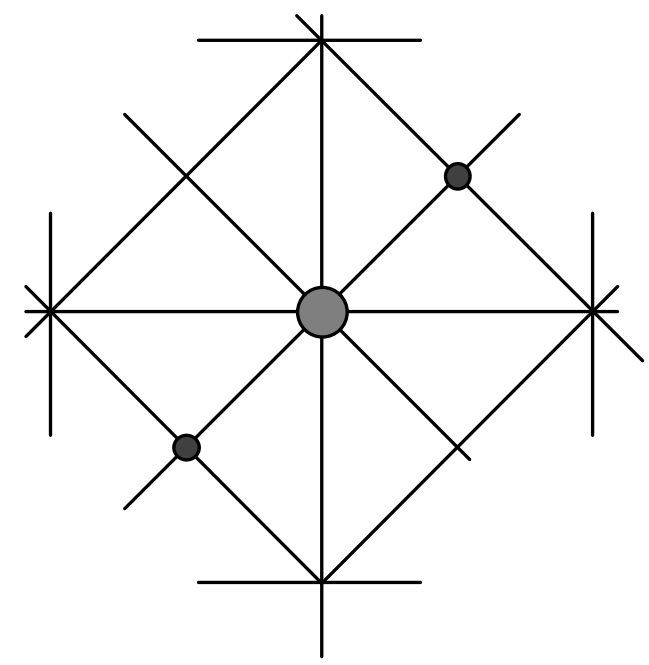

Figura 7.13 - Posições para os dois ou três ou um pontos centrais utilizados na segunda série.

Do mesmo modo que na série anterior, os pontos enfraquecidos possuem um limite elástico inicial 10\% menor que os restantes. 

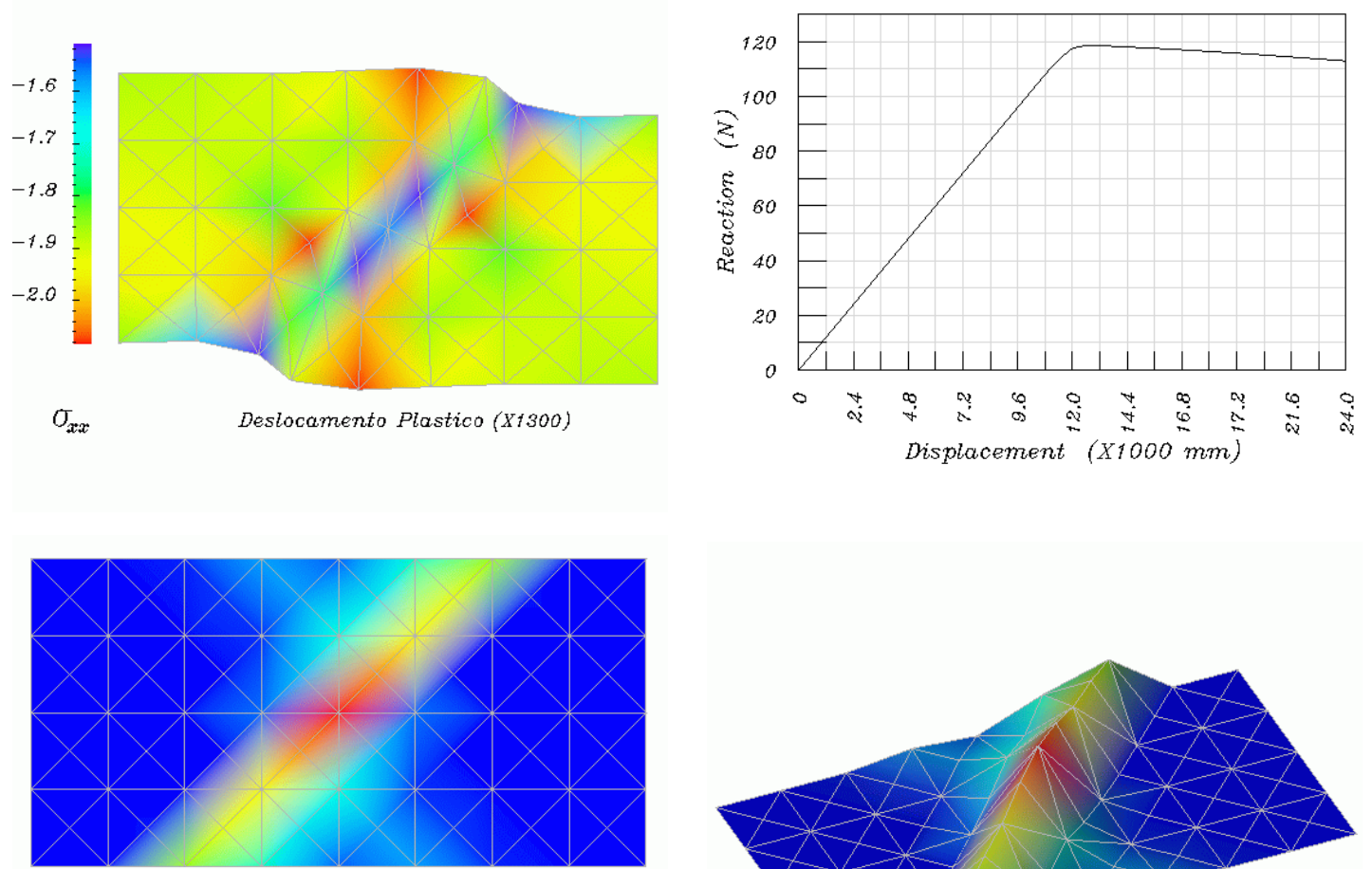

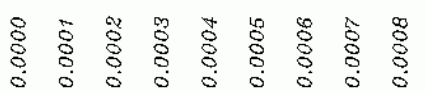

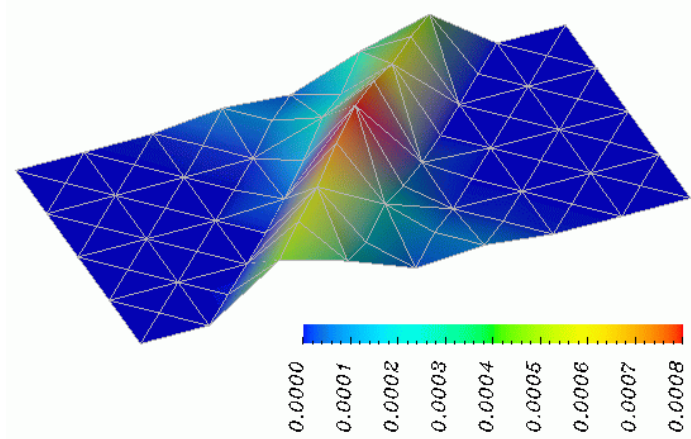

Deformacao equivalente acumulada

Deformacao equivalente acumulada

Figura 7.14 - Série 2: 128 células. Procedimento explícito. Plasticidade local. $\delta=0,024 \mathrm{~mm}, v=0,0.400$ incrementos de carga.

Neste caso a visualização da área com a concentração de deformação é muito mais clara, embora a curva reação-deslocamento seja similar à do exemplo de canto enfraquecido.

Observe-se que os três pontos enfraquecidos se deformam mais. 

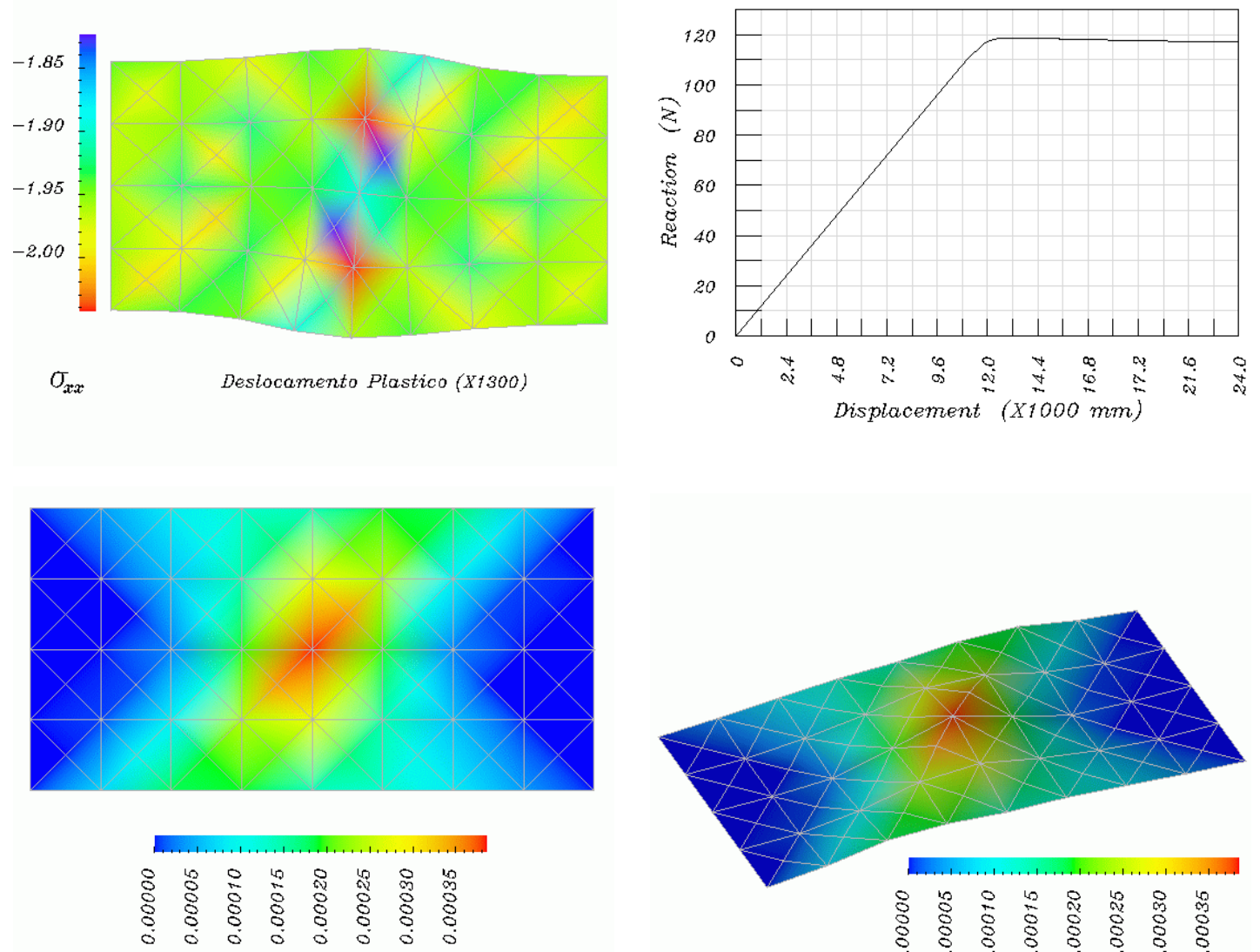

Deformacao equivalente acumulada

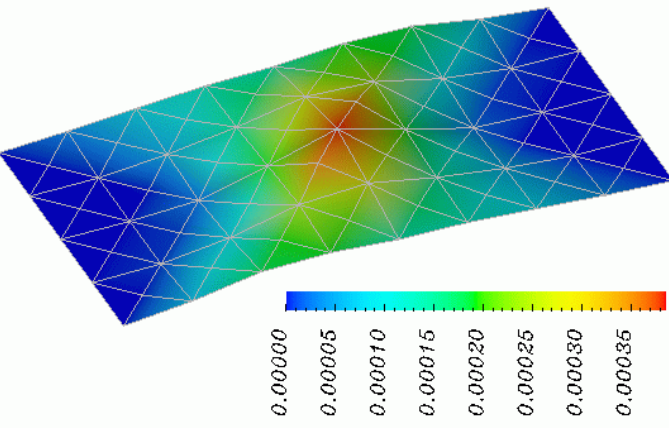

Deformacao equivalente acumulada

Figura 7.15 - Série 2: 128 células. Procedimento explícito. Plasticidade com gradiente. $\delta=0,024 \mathrm{~mm}, v=0,0 . \omega=50000 \mathrm{~N}$. 400 incrementos de carga.

Novamente o efeito regularizador do gradiente pode ser observado. 

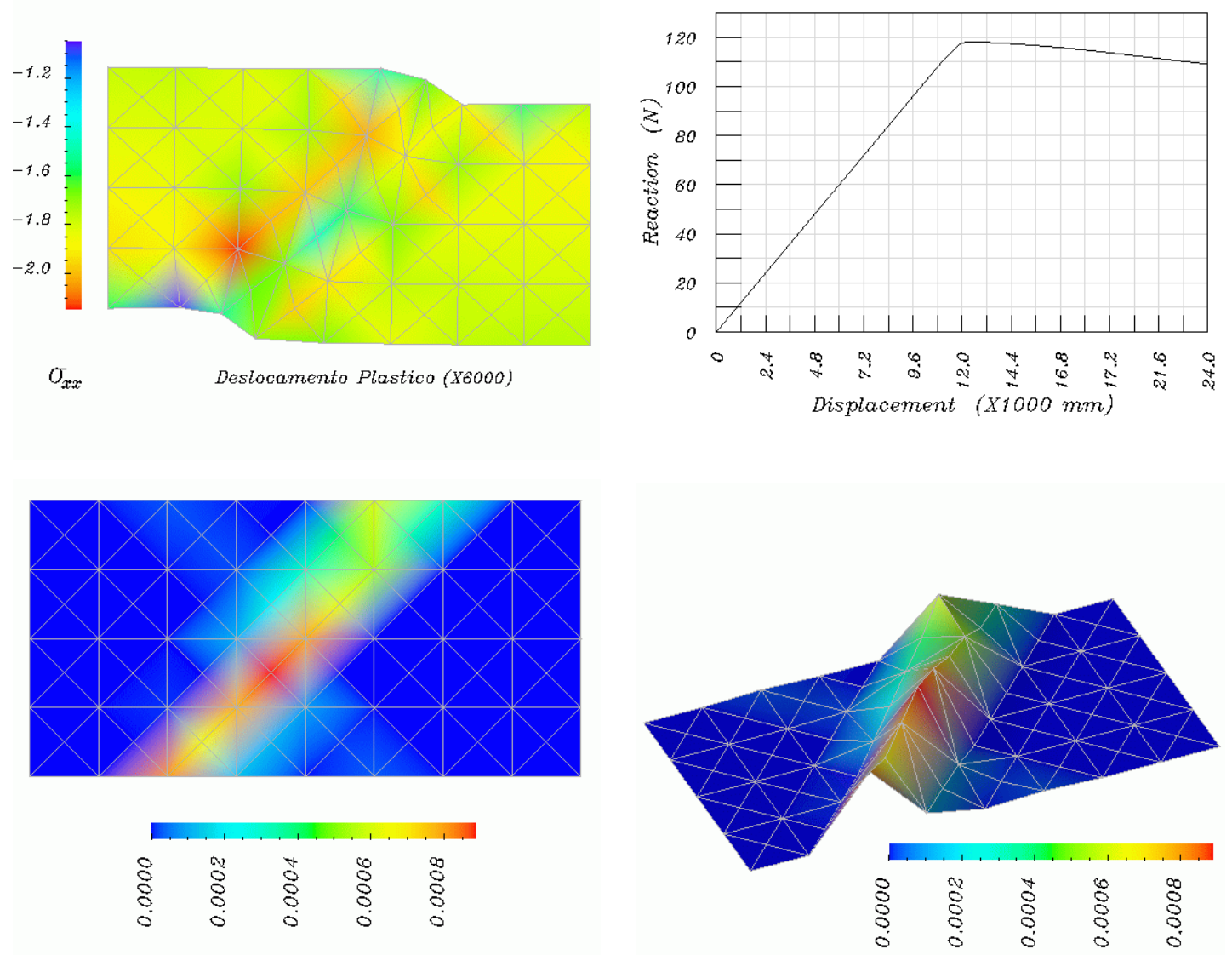

Deformacao equivalente acumulada Deformacao equivalente acumulada

Figura 7.16 - Série 2: 128 células. Procedimento implícito. Plasticidade local. $\delta=0,024 \mathrm{~mm}, v=0,0.400$ incrementos de carga.

Note-se que apenas dois pontos foram enfraquecidos: o central e o da diagonal inferior. Observe-se que a concentração de deformações é mais nítida na metade inferior da faixa.

Ressalte-se ainda que, mesmo tomando-se apenas dois pontos, os resultados obtidos pelo procedimento implícito ainda são melhores que o do explícito local. As deformações quase que se concentram somente na linha diagonal, na metade inferior. 

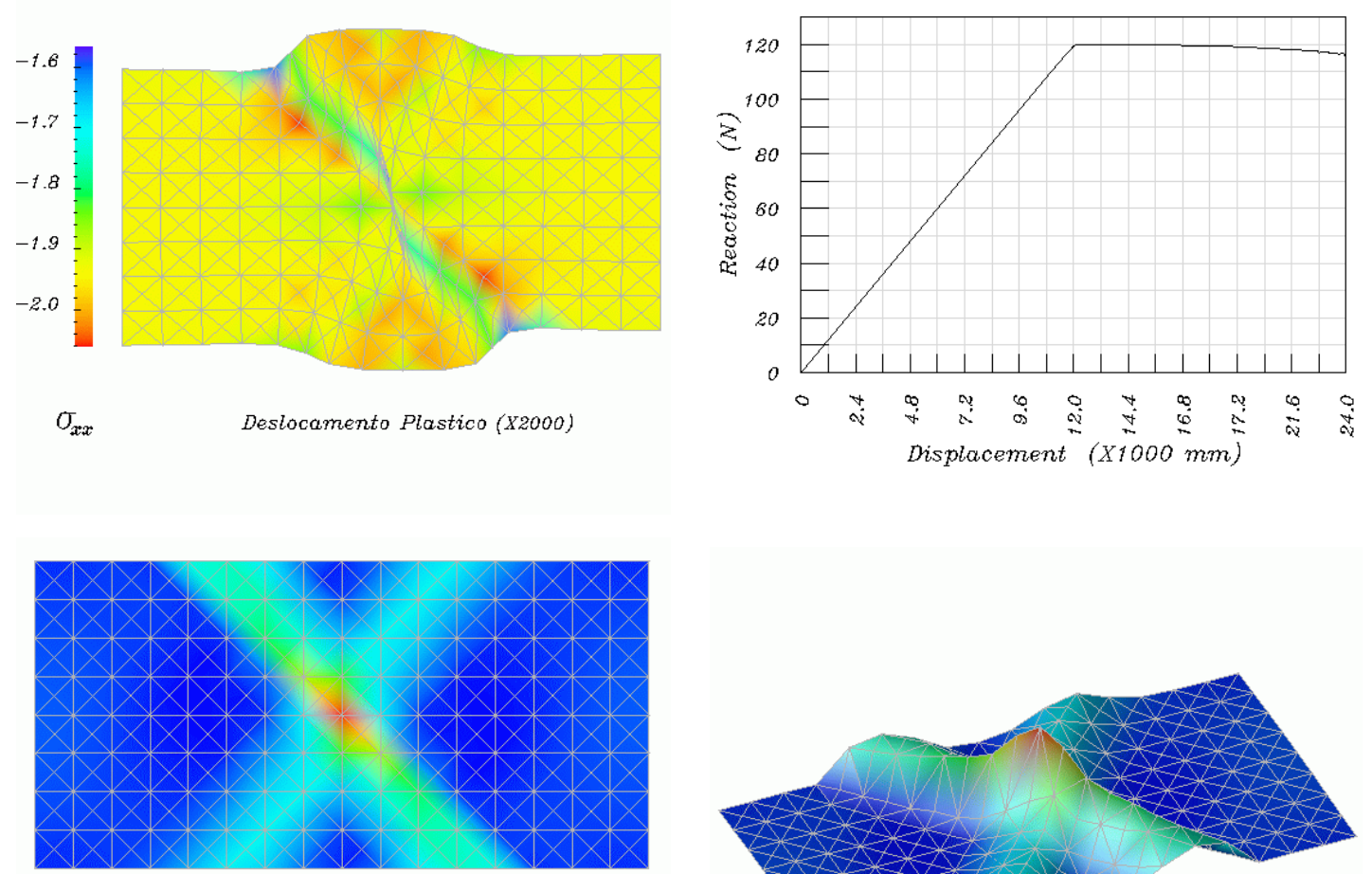

$$
\begin{array}{lllll}
4 & 0 & \infty & 0 \\
8 & 8 & 8 & 8 & 8 \\
8 & 0 & 8 & 8 & 8 \\
0 & 0 & 0 & 0 & 0
\end{array}
$$

Deformacao equivalente acumulada

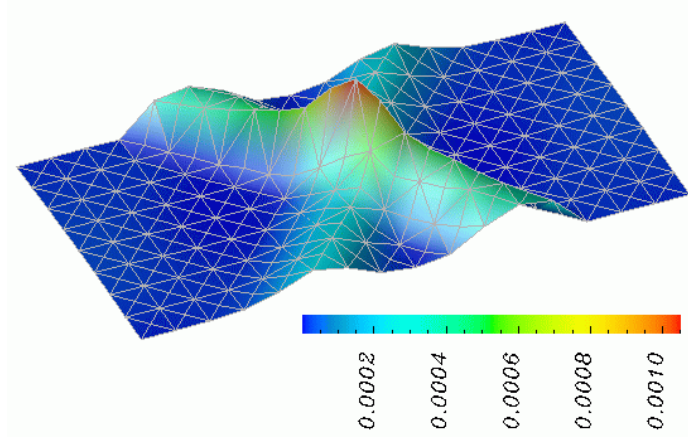

Deformacao equivalente acumulada

Figura 7.17 - Série 2: 512 células. Procedimento explícito. Plasticidade local. $\delta=0,024 \mathrm{~mm}, v=0,0.400$ incrementos de carga.

Neste exemplo a localização não se manifesta tanto na curva reação deslocamento, porque as duas soluções de localização se manifestam fortemente, aumentando assim a átrea de dissipação.

O importante a observar é que, apesar do enfraquecimento ser colocado numa das diagonais, induzindo assim a escolha por uma das soluções simétricas, o procedimento explícito acaba perdendo a maior parte dessa informação inicial. 

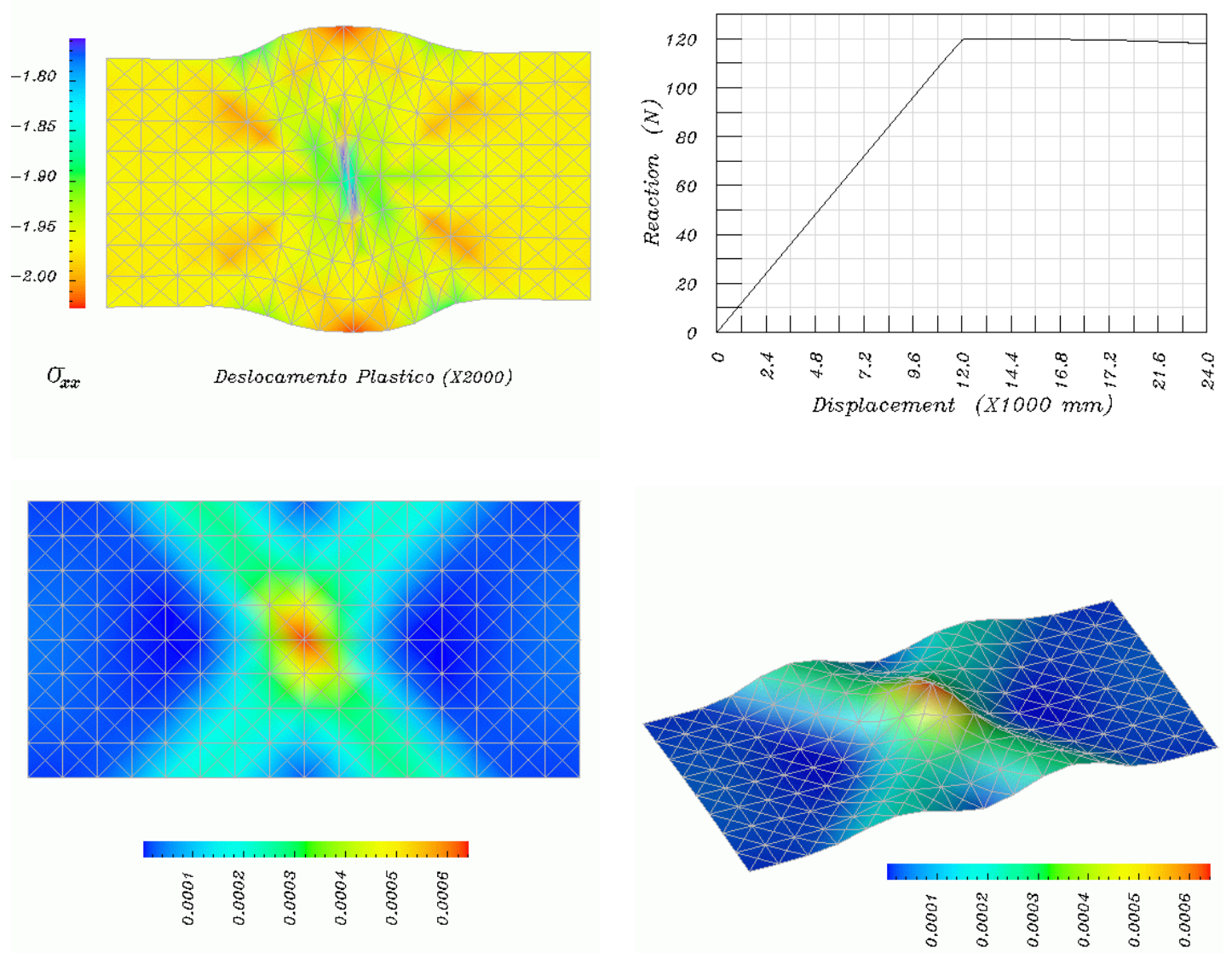

Deformacao equivalente acumulada

Deformacao equivalente acumulada

Figura 7.18 - Série 2: 512 células. Procedimento explícito. Plasticidade com gradiente. $\delta=0,024 \mathrm{~mm}, v=0,0 . \omega=5000 \mathrm{~N}$. 400 incrementos de carga.

Neste exemplo a necessidade de regularização é pequena. Mas está manifesta mais uma vez.

O procedimento explícito confirma sua dificuldade em identificar a solução mais crítica, dando igual ênfase às duas soluções, apesar do enfraquecimento imposto apenas na diagonal, em três pontos. 

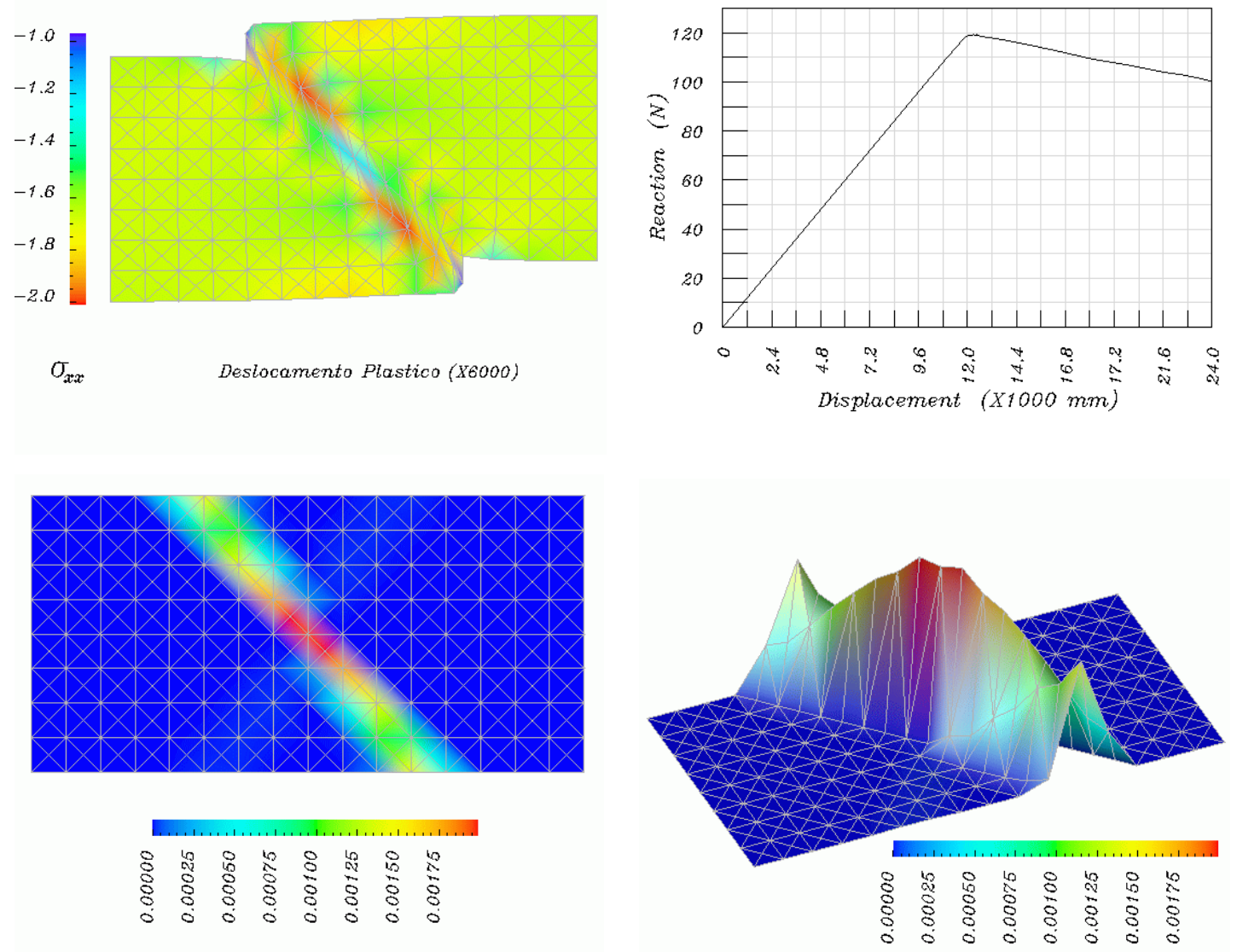

Deformacao equivalente acumulada

Figura 7.19 - Série 2: 512 células. Procedimento implícito. Plasticidade local. $\delta=0,024 \mathrm{~mm}, v=0,0.400$ incrementos de carga.

Mais uma vez, os resultados obtidos pelo procedimento implícito mostram um desempenho bem mais satisfatório. Somente a solução indicada pelos pontos enfraquecidos é capturada, com total clareza. 

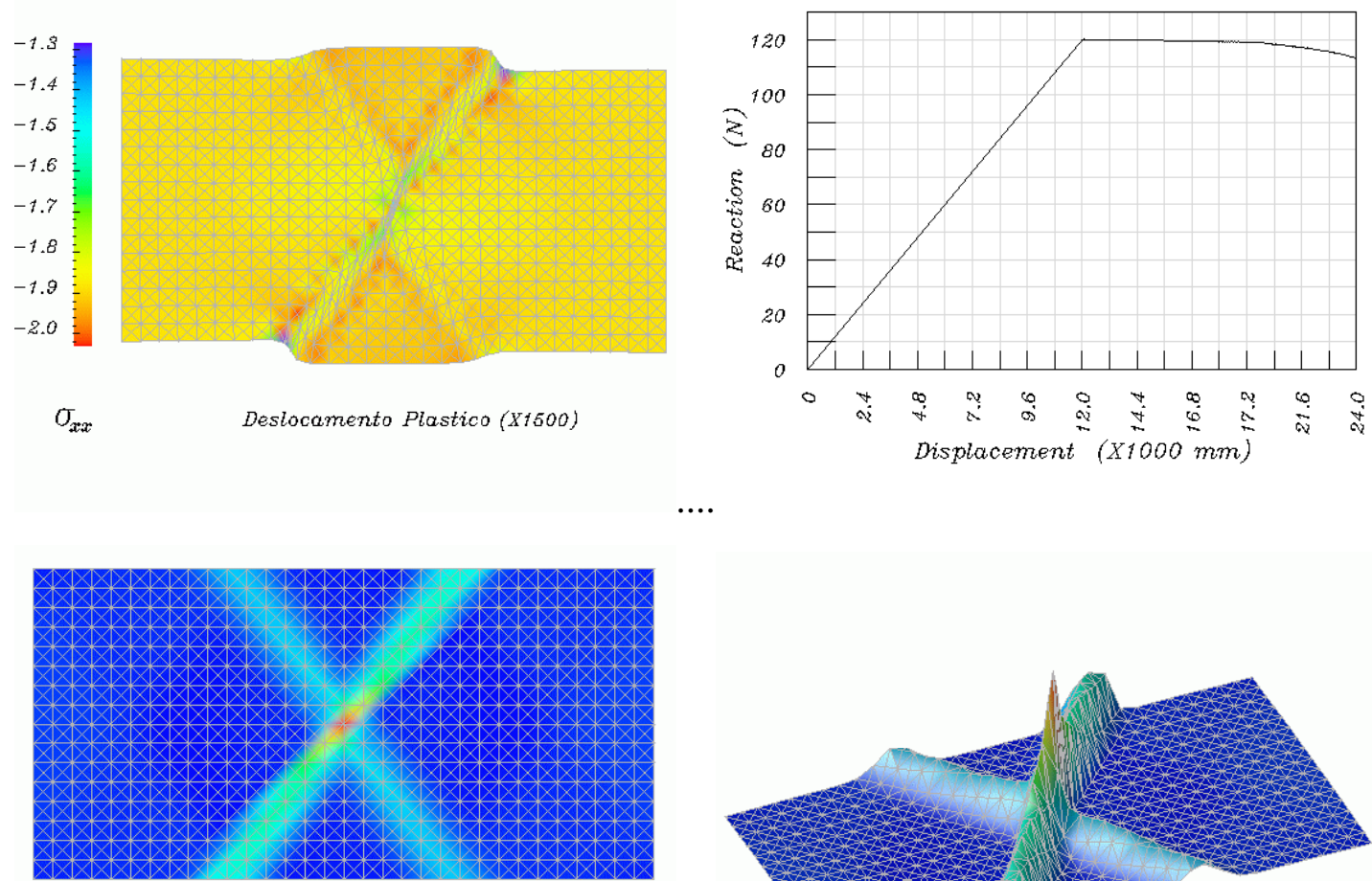

$$
\begin{array}{llllll} 
& 0 & 0 & 0 & 4 & 0 \\
0 & 8 & 0 & 8 & 0 & 0 \\
8 & 8 & 8 & 8 & 5 & 0 \\
0 & 0 & 0 & 8 & 0 & 0 \\
0 & 0 & 0 & 0 & 0 & 0
\end{array}
$$

Deformacao equivalente acumulada

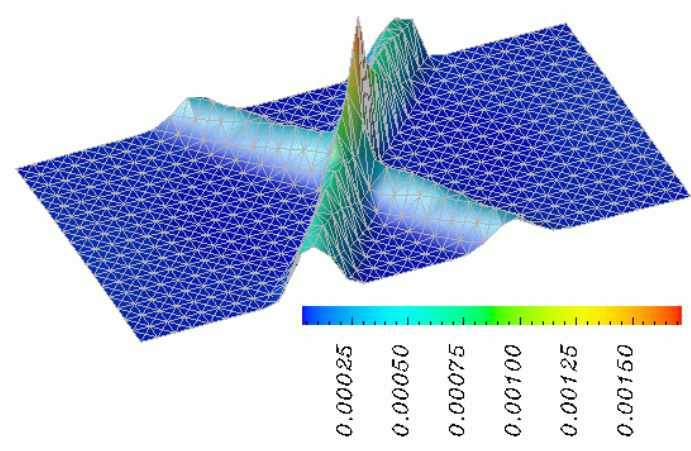

Deformacao equivalente acumulada

Figura 7.20 - Série 2: 2048 células. Procedimento explícito. Plasticidade local. $\delta=0,024 \mathrm{~mm}, v=0,0.400$ incrementos de carga.

Neste caso o método explícito se sai melhor, fazendo uma nítida escolha pela solução indicada pelos três pontos enfraquecidos em uma das diagonais, apesar de ainda permitir que a segunda solução se manifeste com uma certa intensidade. Há uma melhora geral dos resultados. 

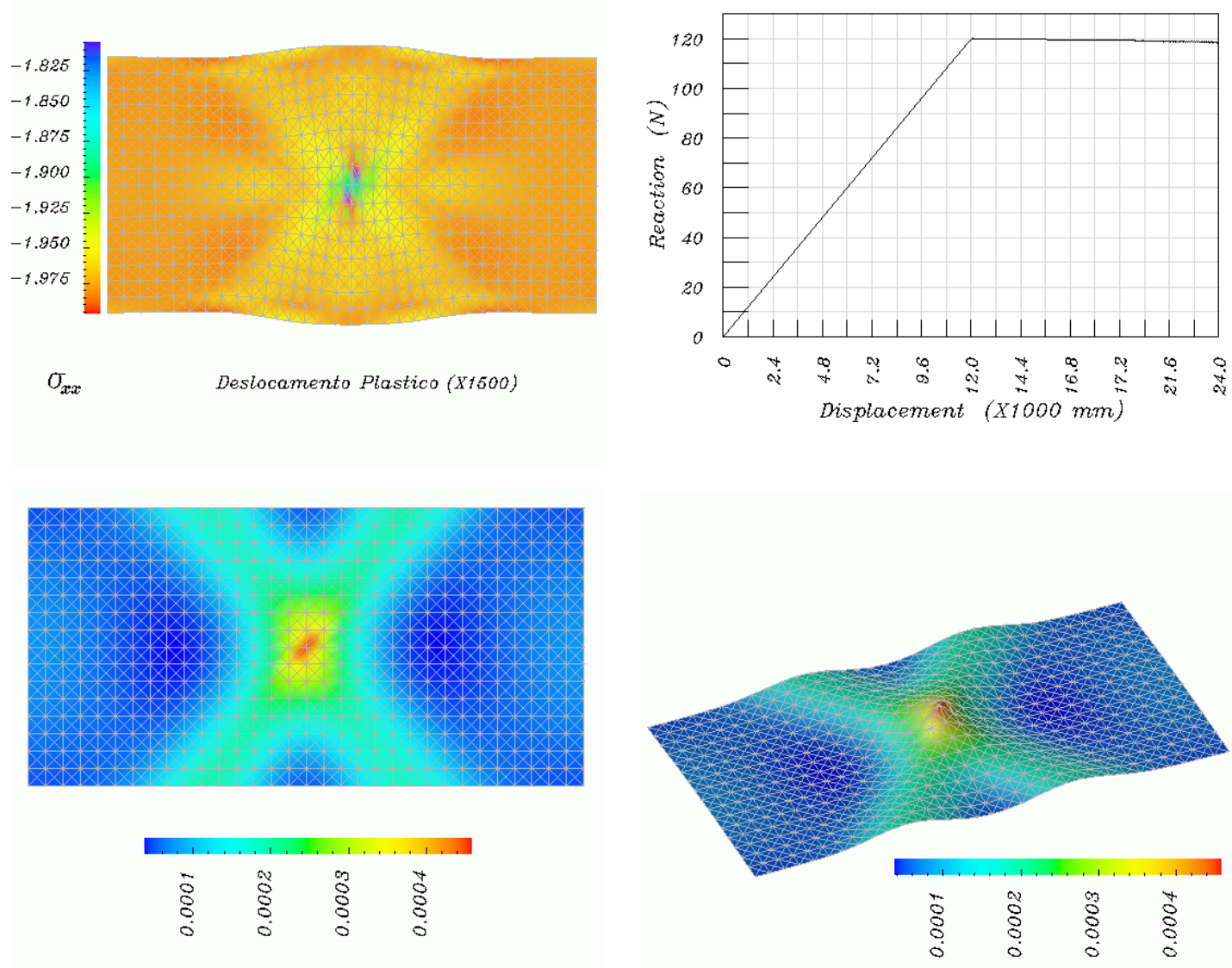

Deformacao equivalente acumulada

Deformacao equivalente acumulada

Figura 7.21 - Série 2: 2048 células. Procedimento explícito. Plasticidade com gradiente. $\delta=0,024 \mathrm{~mm}, v=0,0 . \omega=5000 \mathrm{~N}$. 400 incrementos de carga.

Como neste caso o procedimento explícito desempenha melhor seu papel, o gradiente também tem uma boa oportunidade de executar o seu, conseguindo espalhar a faixa de deformação e agindo conforme esperado. 

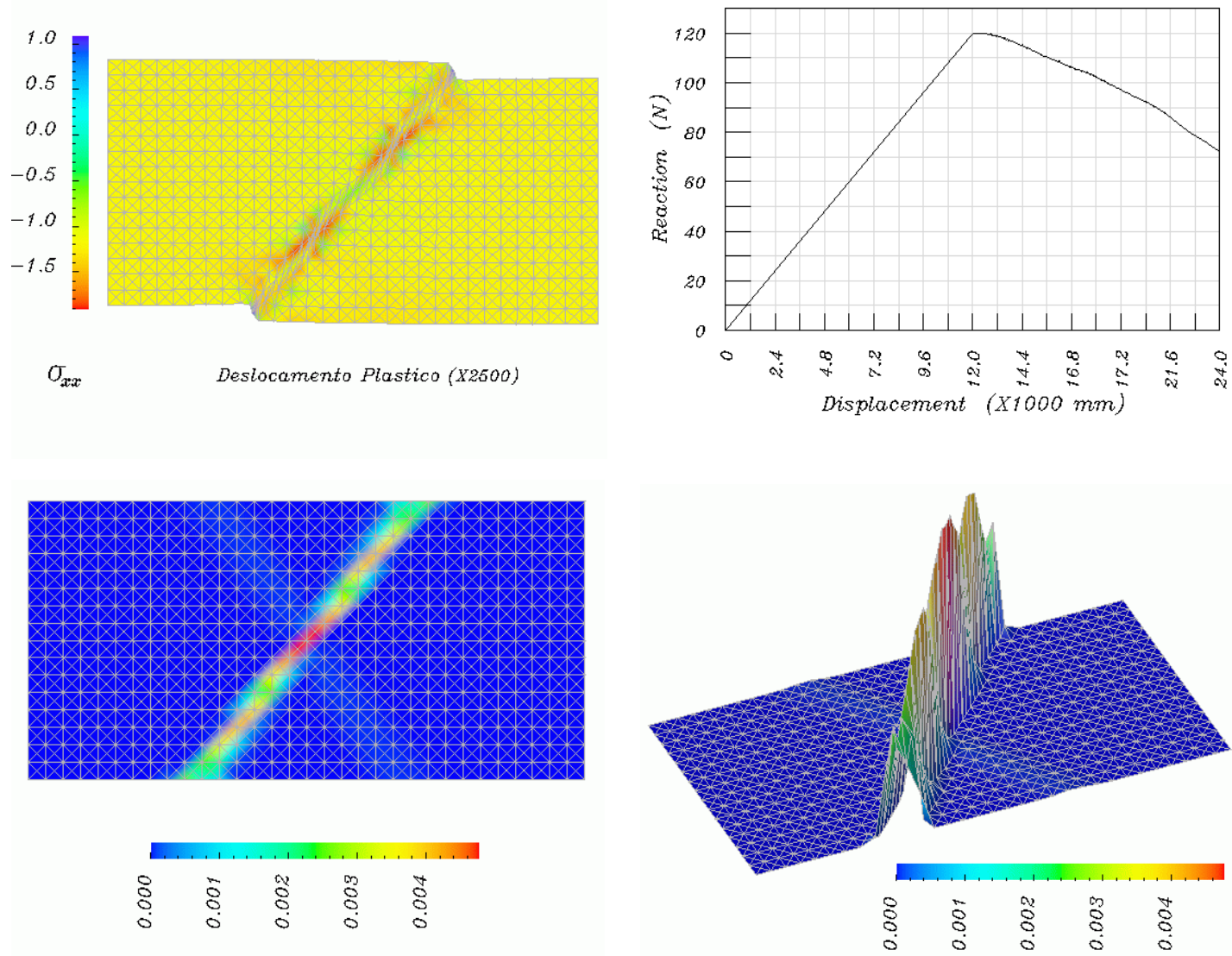

Deformacao equivalente acumulada

Deformacao equivalente acumulada

Figura 7.22 - Série 2: 2048 células. Procedimento implícito. Plasticidade local. $\delta=0,024 \mathrm{~mm}, v=0,0.400$ incrementos de carga.

Novamente o método implícito retorna exatamente a resposta esperada. 

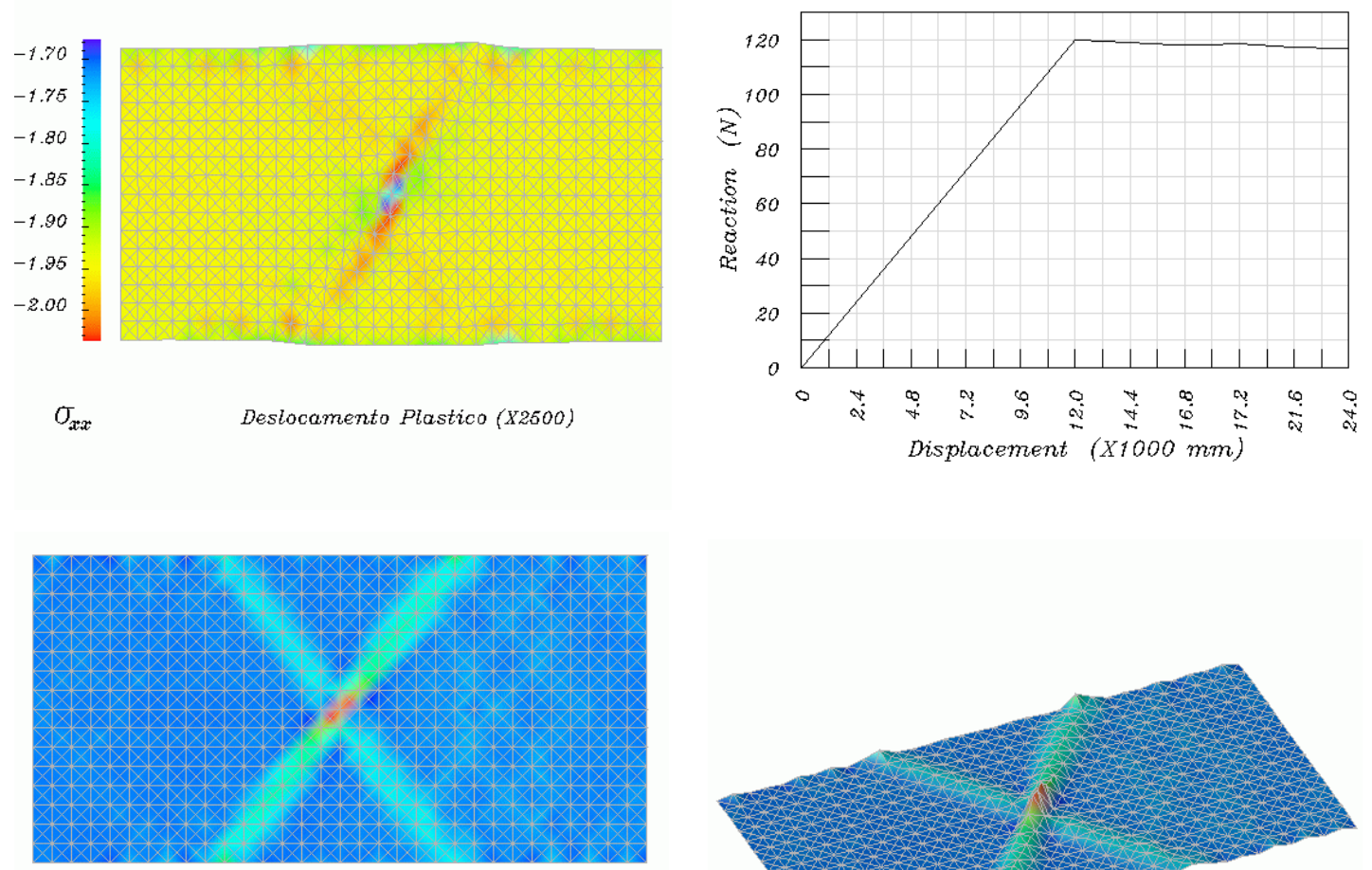

$$
\begin{array}{lllll}
5 & 0 & 0 & 0 & 0 \\
8 & 8 & 8 & 8 & 8 \\
8 & 0 & 0 & 0 & 0 \\
0 & 0 & 0 & 0 & 0
\end{array}
$$

Deformacao equivalente acumulada

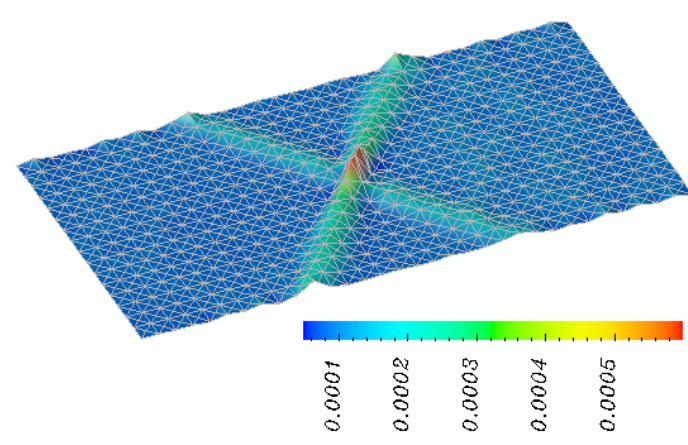

Deformacao equivalente acumulada

Figura 7.23 - Série 2: 2048 células. Procedimento implícito. Plasticidade local. $\delta=0,024 \mathrm{~mm}, v=0,0.10$ incrementos de carga.

Esta figura mostra os resultados pelo método implícito utilizando-se apenas 10 passos de carregamento. Só abaixo de 20 incrementos esse exemplo as duas soluções simétricas manifestaram-se de forma comparável. Os resultados são apenas um pouco piores do que os obtidos pelo procedimento explícito com 400 incrementos. Observe-se nas imagens de deformação equivalente que o incremento é tão grande que pode-se notar que a iniciação de novas linhas de localização surgem quase por toda parte, especialmente do lado direito do retângulo, indicando pura perturbação numérica no processo. 

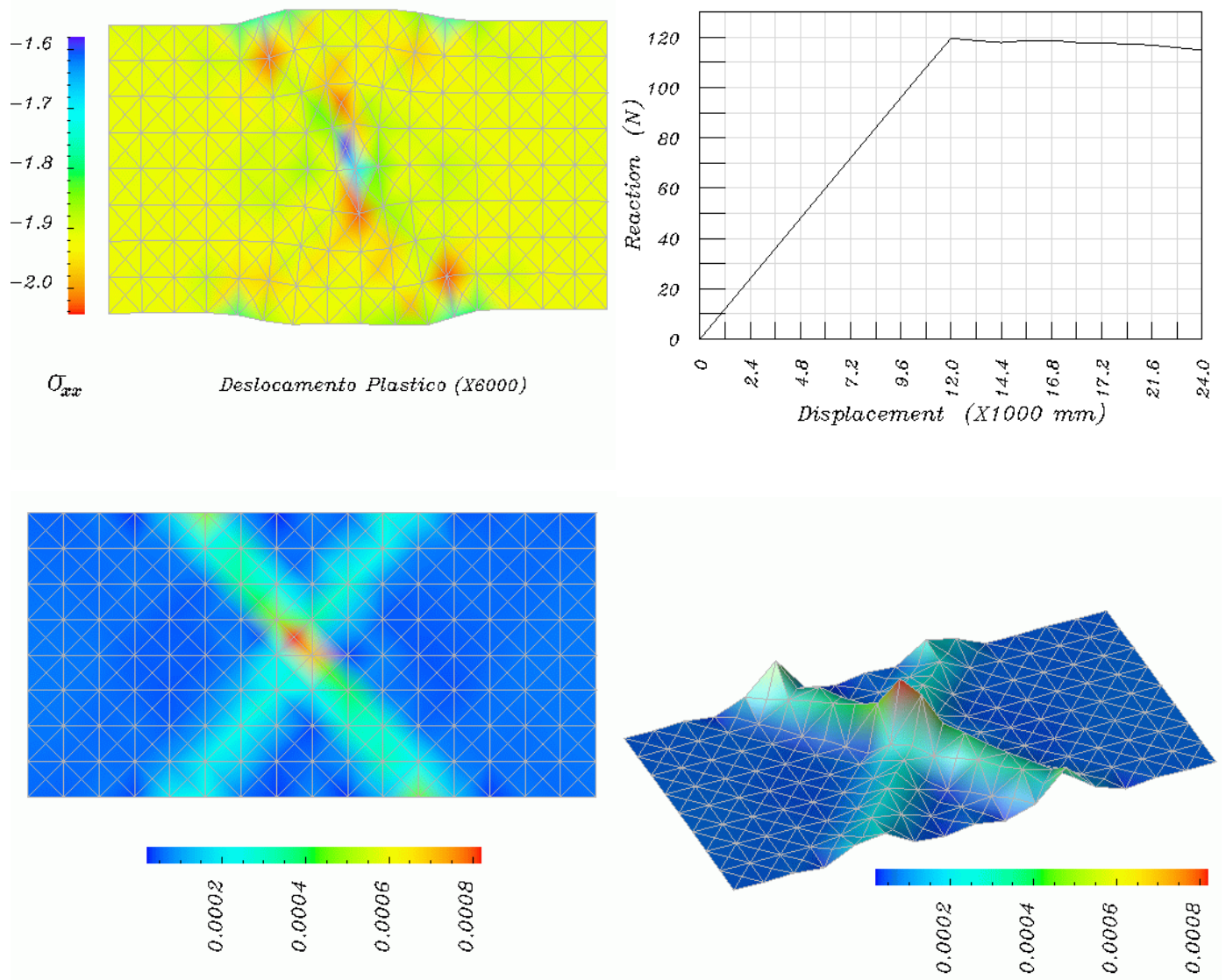

Deformacao equivalente acumulada

Deformacao equivalente acumulada

Figura 7.24 - Série 2: 512 células. Procedimento implícito. Plasticidade local. $\delta=0,024 \mathrm{~mm}, v=0,0.20$ incrementos de carga.

Como no caso da malha de 2048 células, apenas aumentando-se muito o tamanho do incremento observa-se a manifestação das duas faixas, quando é utilizado o método implícito. Além disso, apenas dois pontos são enfraquecidos. 

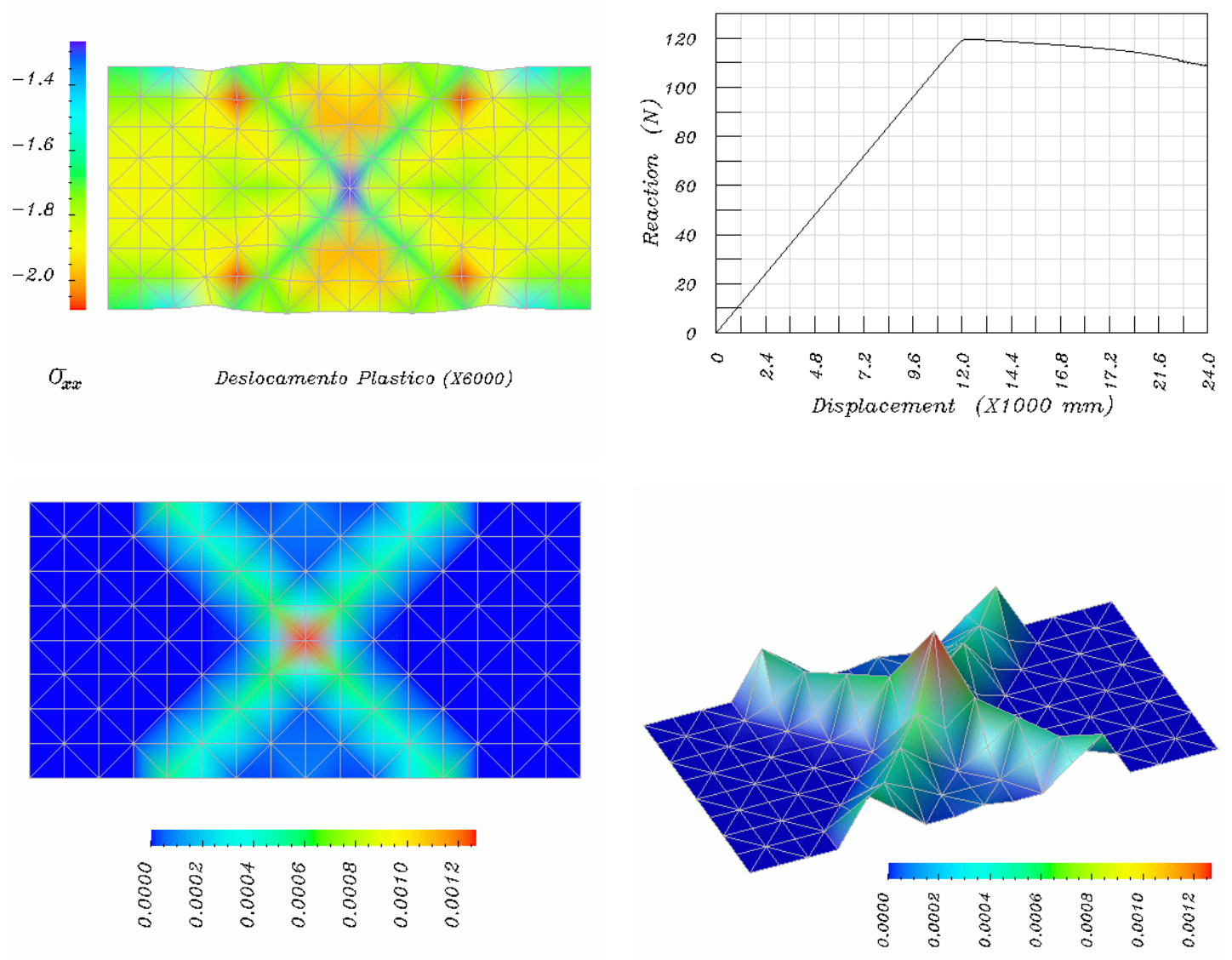

Deformacao equivalente acumulada

Deformacao equivalente acumulada

Figura 7.25 - Série 2: 256 células. Procedimento implícito. Plasticidade local. $\delta=0,024 \mathrm{~mm}, v=0,0.400$ incrementos de carga.

Neste exemplo ilustrativo, apenas o nó central é enfraquecido. Como através de um único nó não é possível quebrar a simetria, o resultado consistentemente apresenta as duas soluções igualmente críticas. 


\subsection{Terceira série: procedimentos implícitos para a plasticidade com gradiente}

Nesta série é utilizado o mesmo retângulo da segunda série, também com enfraquecimento na região central. Na primeira parte, resultados obtidos utilizandose operador tangente consistente para a plasticidade com gradiente são comparados com os da plasticidade local. $\mathrm{Na}$ segunda parte, apenas como ilustração complementar, a mesma comparação é feita utilizando-se operador contínuo para a plasticidade com gradiente, mostrando o trabalho realizado.

\subsubsection{Operador tangente consistente}
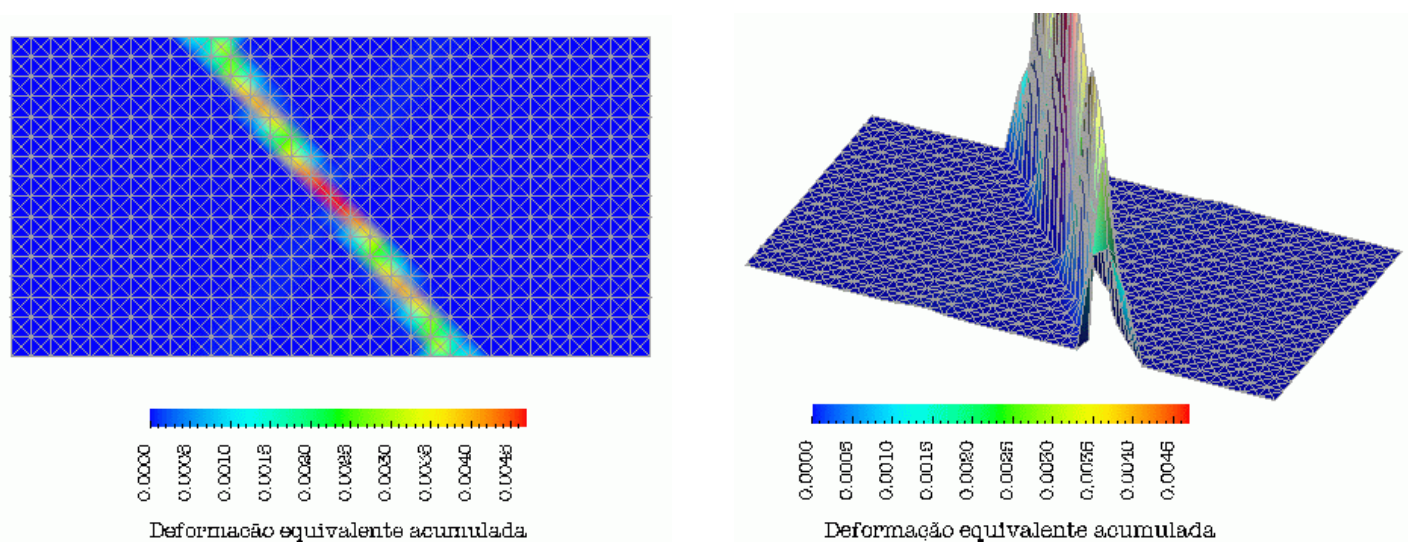

Figura 7.26 - Série 3: w=0,01 N; três pontos enfraquecidos no centro da diagonal; 2048 elementos.

Esta figura representa a referência para as próximas três seguintes. $\mathrm{O}$ comportamento local é simulado utilizando-se um valor muito pequeno para o parâmetro w. Trata-se praticamente do mesmo resultado apresentado na Figura 7.22. 

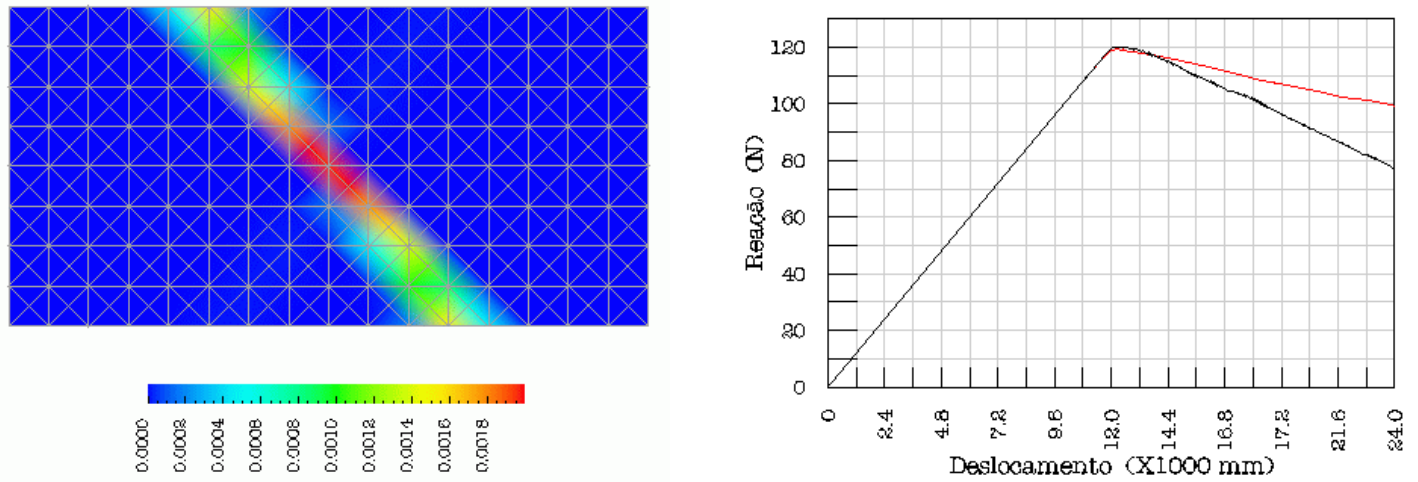

2048 (em preto) X 512 elementos

Figura 7.27 - Série 3: w=0,01 N; três pontos enfraquecidos no centro da diagonal; 512 elementos. Comparada com a malha de 2048 elementos (em preto) (ver Figura 7.26).
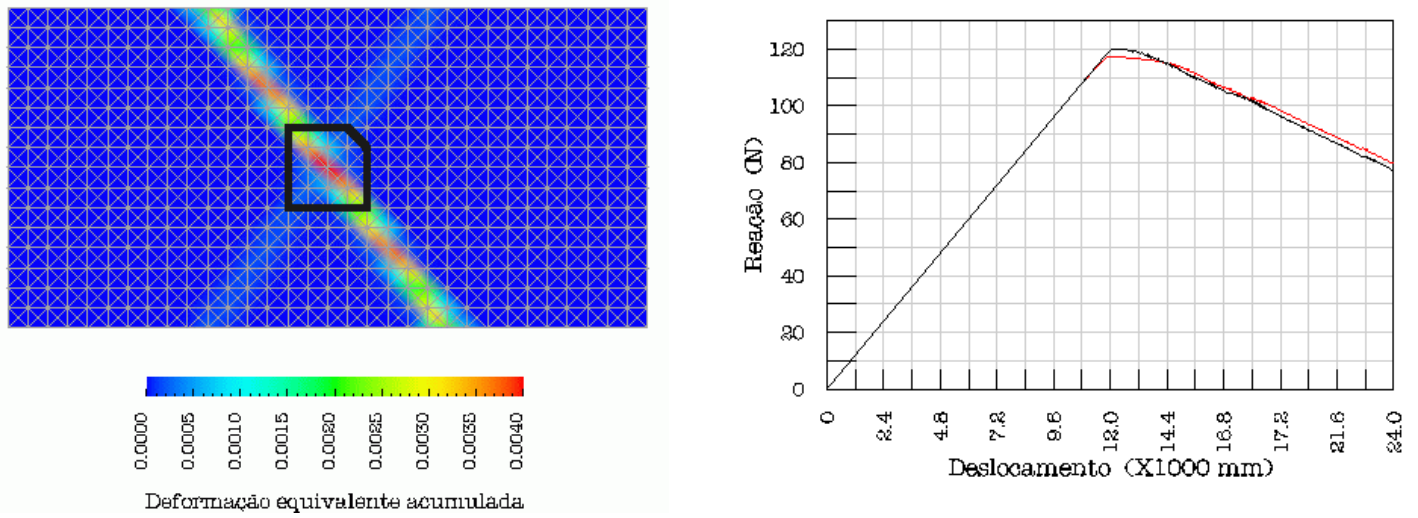

2048 elementos: 3 pontos (em preto) X quadrado (sem 1 vértice)

Figura 7.28 - Série 3: w=0,01 N; enfraquecimento no quadrado assinalado (exceto num vértice).

Nesta figura todo o quadrado assinalado é enfraquecido, exceto no vértice superior direito. 2048 elementos. Novamente a comparação é feita com o caso local da Figura 7.26 (em preto). Apesar da área enfraquecida ser bem maior, o resultado é praticamente o mesmo, mostrando que o tamanho do defeito não é importante, desde que seja bastante menor que as dimensões da estrutura, como neste caso. 


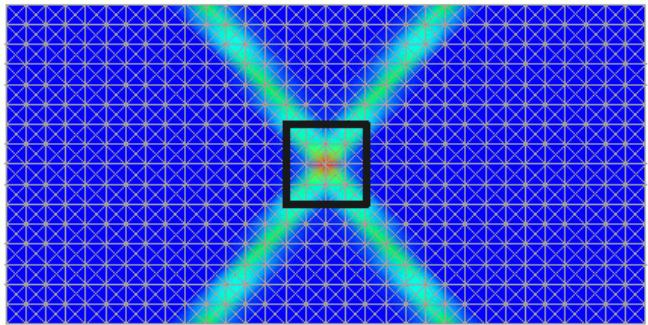

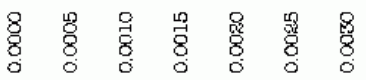

Deformacāo equivalente acumulada

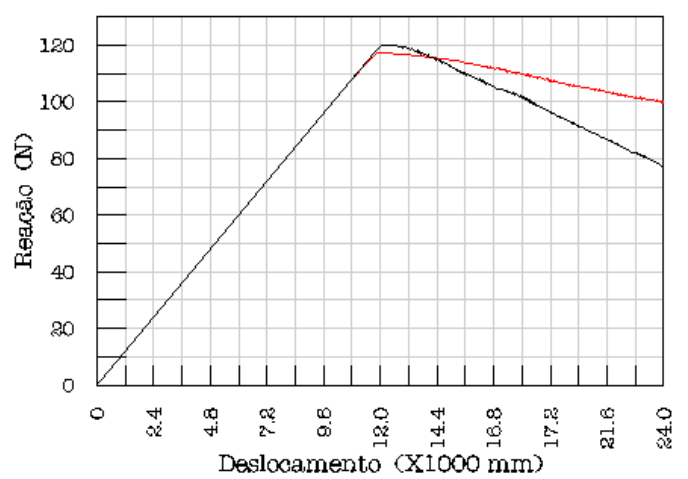

2048 elementos: 3 pontos (em preto) X quadrado

Figura 7.29 - Série 3: w=0,01 N; enfraquecimento no quadrado assinalado (simetria).

A mesma referência das figuras anteriores é mantida (em preto). Neste caso, a simetria é mantida, e em consequiência as duas soluções críticas são mostradas.
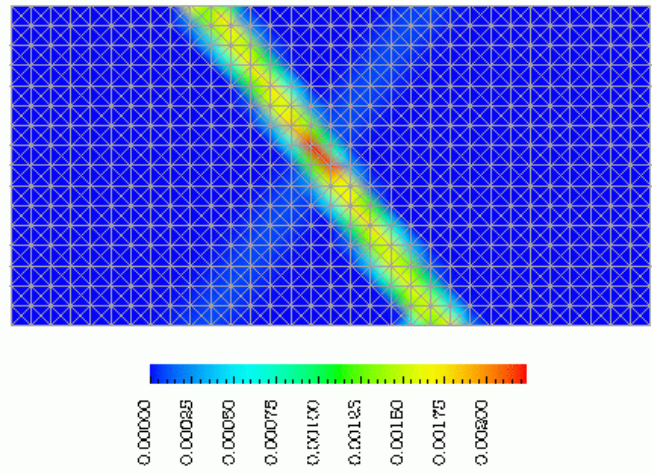

Deformaçào equivalente acumulada

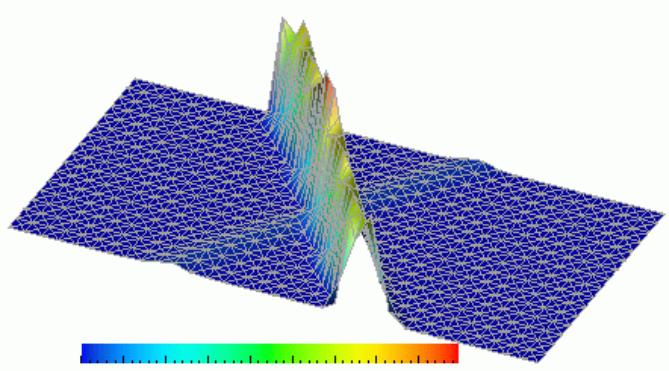

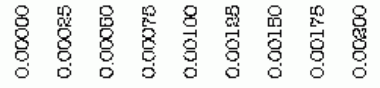

Deformacáo equivalente acumulada

Figura 7.30 - Série 3: w=500 N; 2048 elementos

O exemplo desta figura será usado como referência nas duas seguintes. 

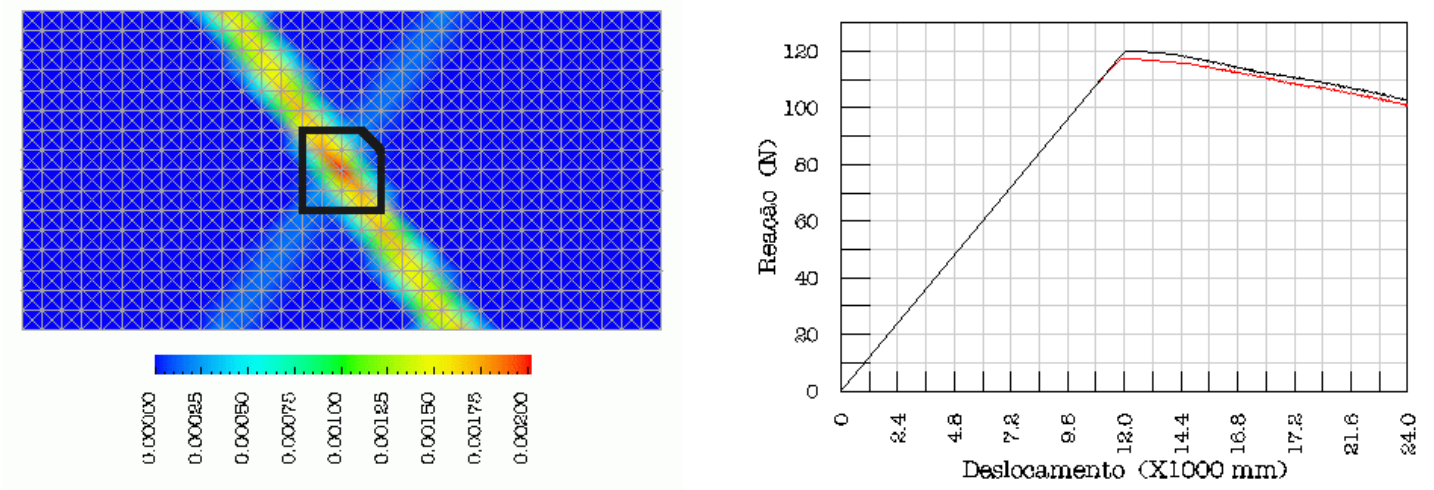

Deformaçāo equivalente acumulada

w=500, 2048 elementos: 3 pontos (em preto) X quadrado (sem 1 vértice)

Figura 7.31 - Série 3: w=500 N; 2048 elementos (quadrado enfraquecido - menos 1 vértice)

Novamente a comparação com o resultado de a área enfraquecida menor da figura anterior (em preto no gráfico deslocamento $X$ reação), mostra influência desprezível do tamanho da região enfraquecida.
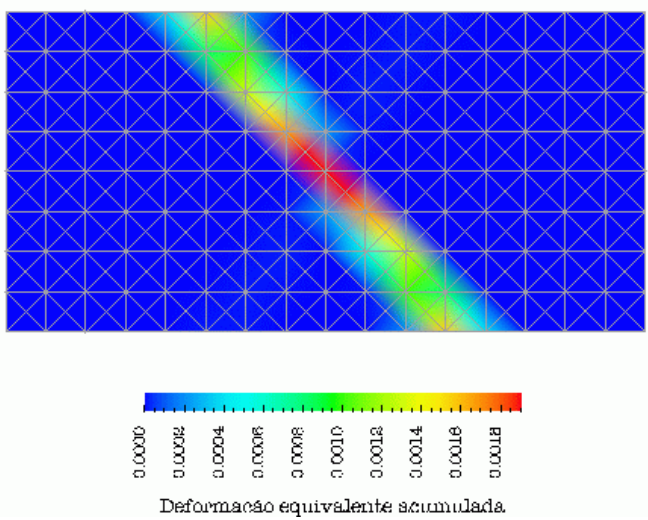

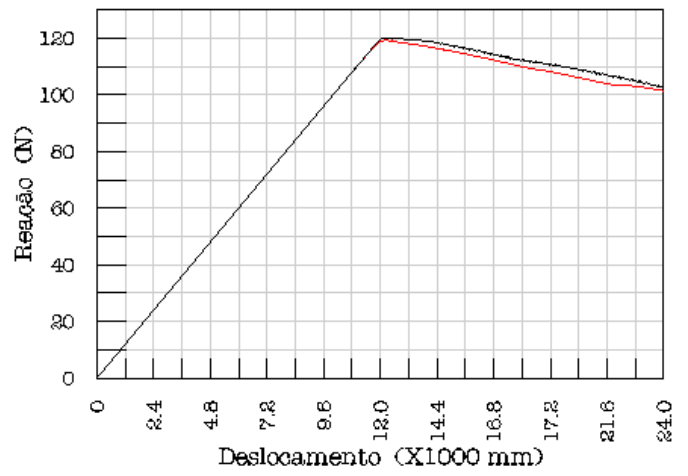

$\mathrm{W}=500: 2048$ (em preto) X 512 elementos

Figura 7.32 - Série w=500 N; 512 elementos.

O exemplo da Figura 7.30 é novamente tomado como referência (em preto). Devido à presença do gradiente, a diferença dos resultados entre os dois tamanhos de malha é muito pequena. 


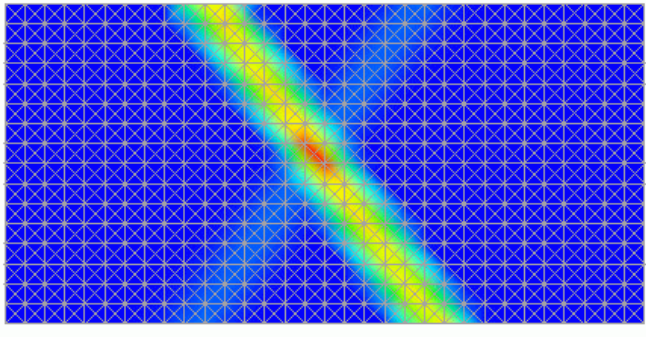

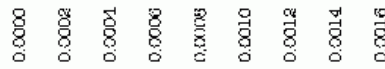

Deformacāo equivalente acumulada

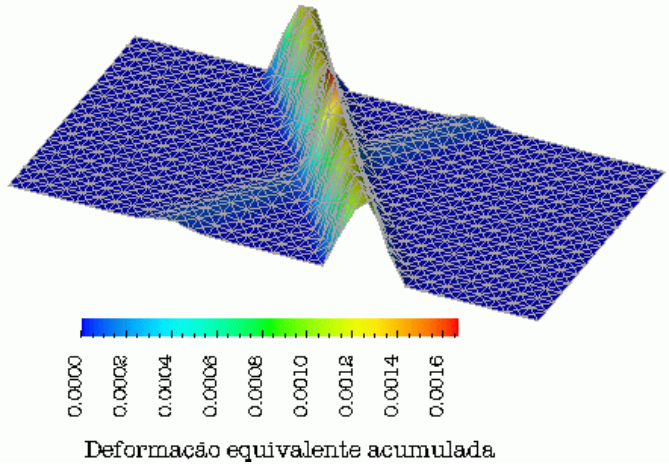

Figura 7.33 - Série 3: w=1000 N; 2048 elementos.

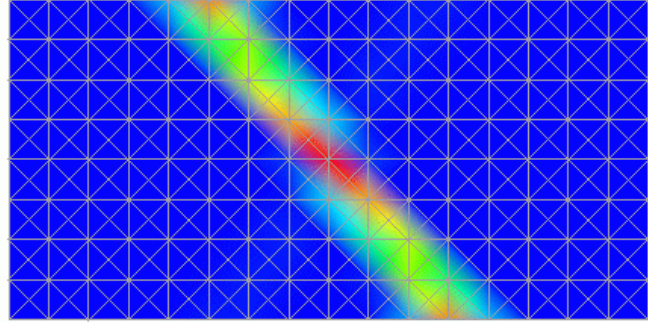

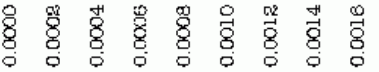

Deformacāo equiva,lente acumulada

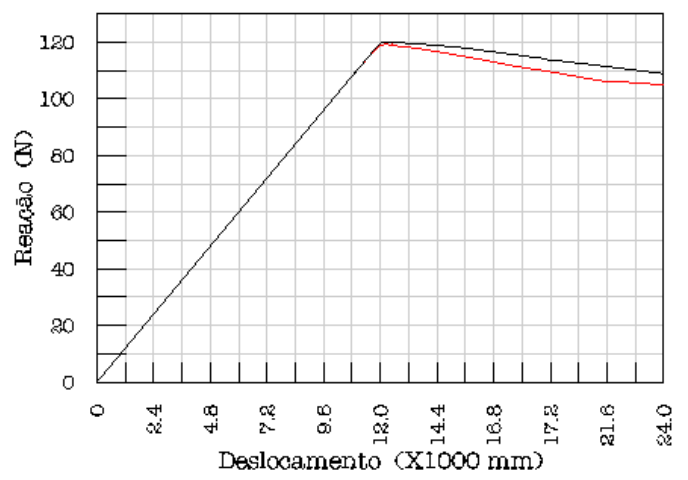

$\mathrm{W}=1000: 2048$ (em preto) X 512 elementos

Figura 7.34 - Série 3: w=1000 N; 512 elementos.

A comparação de resultados de malhas diferentes mostra que a influência do tamanho das malhas é bem pequena, na presença do gradiente. 


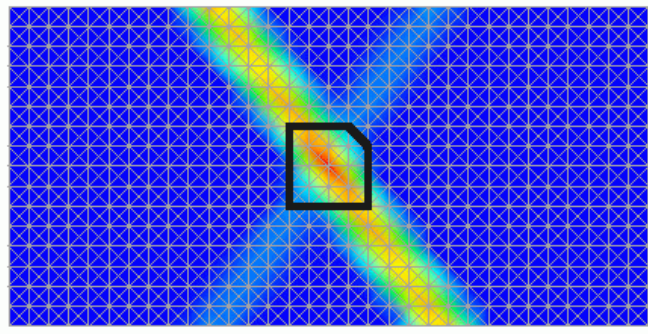

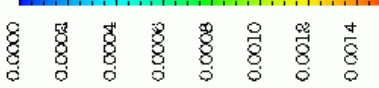

Deformaçăo equivalente acumulada

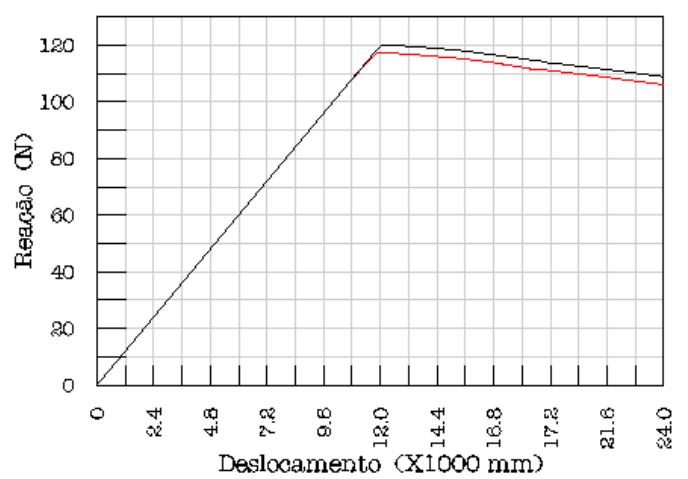

$\mathrm{w}=1000,2048$ elementos: 3 pontos (em preto) $\mathrm{X}$ quadrado (sem 1 vértice)

Figura 7.35 - Série 3: w=1000 N; 2048 elementos (quadrado enfraquecido - menos 1 vértice).

Exceto pelo ponto de início de plastificação, o tamanho da área enfraquecida não exerce influência significativa nos resultados.
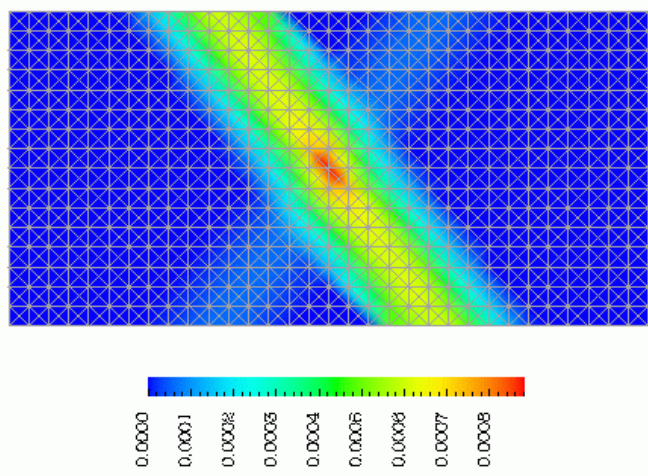

Deformaça equivalente acumulada

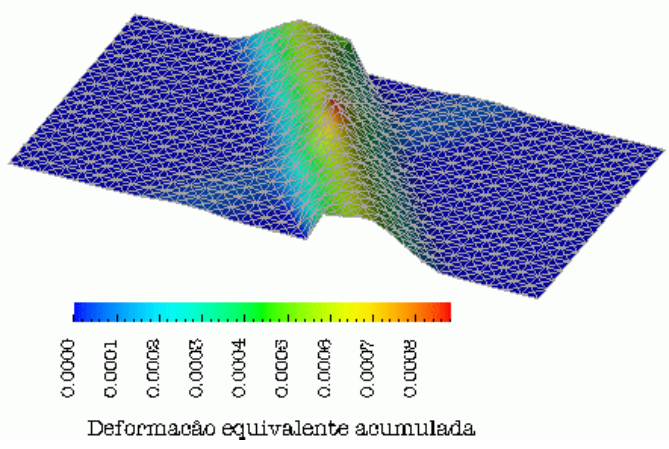

Deformacaio equivalente acumulada

Figura 7.36 - Série 3: w=5000 N; 2048 elementos.

É a referência para a figura seguinte. 

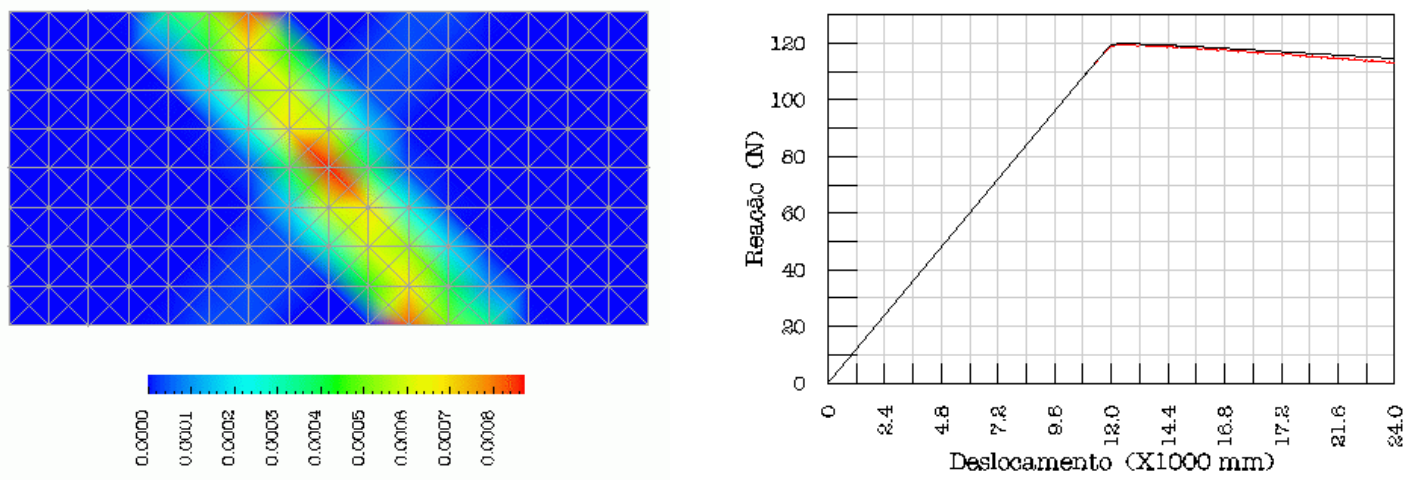

$\mathrm{W}=5000: 2048$ (em preto) X 512 elementos

Figura 7.37 - Série 3: w=5000 N; 512 elementos.

Com o aumento do valor do parâmetro w, a diferença de resultados nas duas malhas é ainda menor. Os resultados praticamente coincidem.

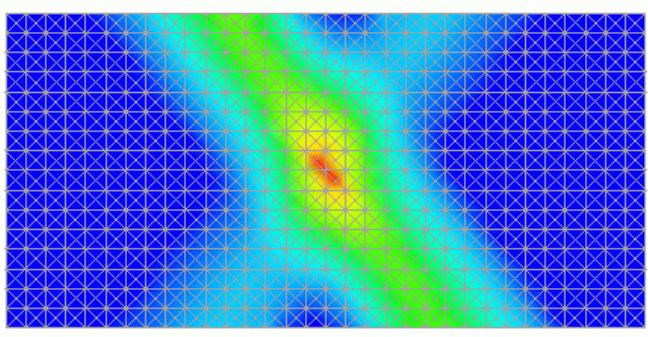

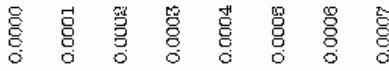

Deformacào equivalente acumulada

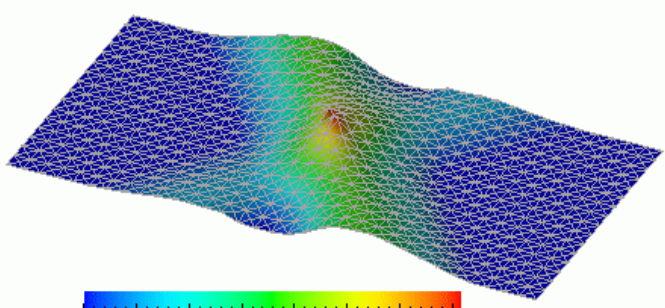

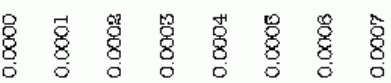

Deformacăo equivalente acumulada

Figura 7.38 - Série 3: w=10000 N; 2048 elementos.

Referência para a figura seguinte. 

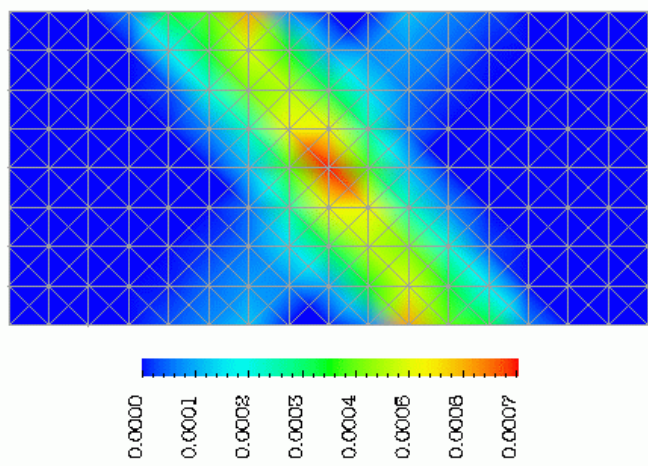

Deformaçāo equivalente acumulada

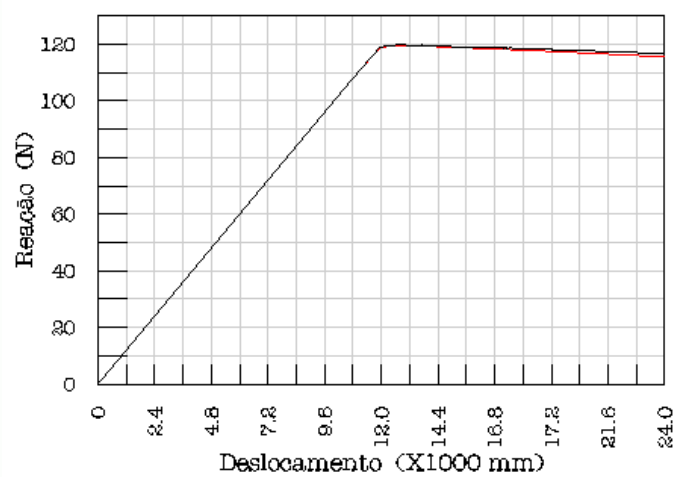

$\mathrm{W}=10000: 2048$ (em preto) X 512 elementos

Figura 7.39 - Série 3: w=10000 N; 512 elementos.

Novamente a diferença de resultados entre as malhas é insignificante.

Nos exemplos anteriores o encruamento negativo (h) é muito pequeno para que se possa verificar o efeito da localização nos gráficos de deslocamento contra reação na extremidade. Aumentando-se o valor da constante de encruamento obtémse um efeito visível.
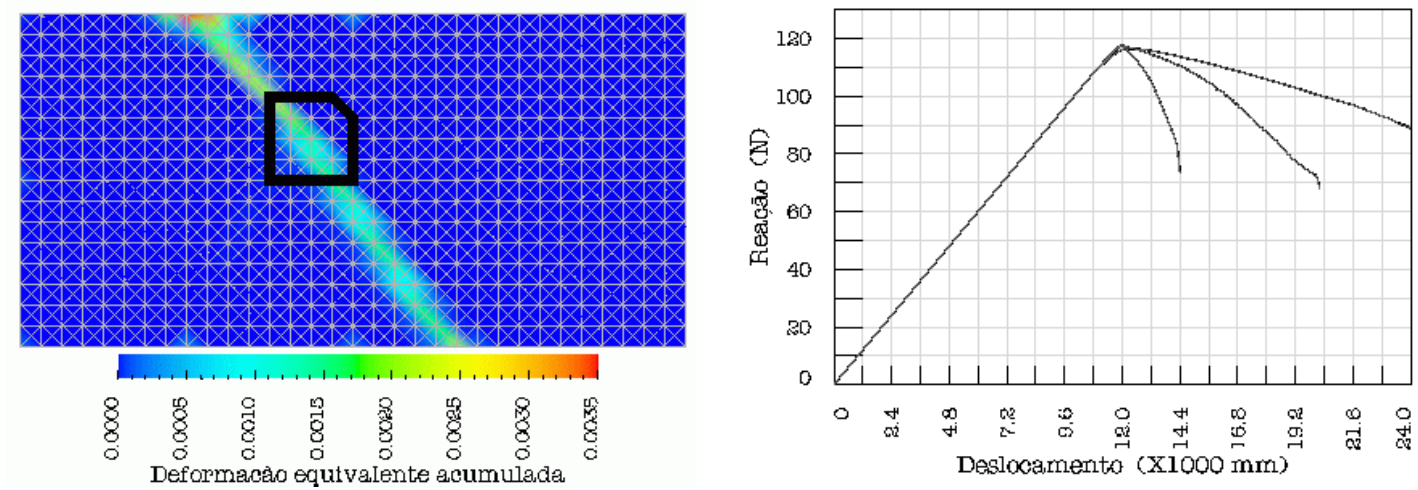

$\mathrm{h}=0.025 \mathrm{E}$, local: 2048,512 e 128 elementos

Figura 7.40 - Série 3: Plasticidade local, $h=-0.025$ E.

Os gráficos deslocamento $\mathrm{X}$ reação evidenciam a dependência dos resultados em relação ao tamanho da malha, conforme já mostravam a Figura 7.16, a Figura 7.19 e a Figura 7.22, em conjunto. Quanto menor o tamanho da malha, menor a área de dissipação de energia, resultando numa reação menor. 

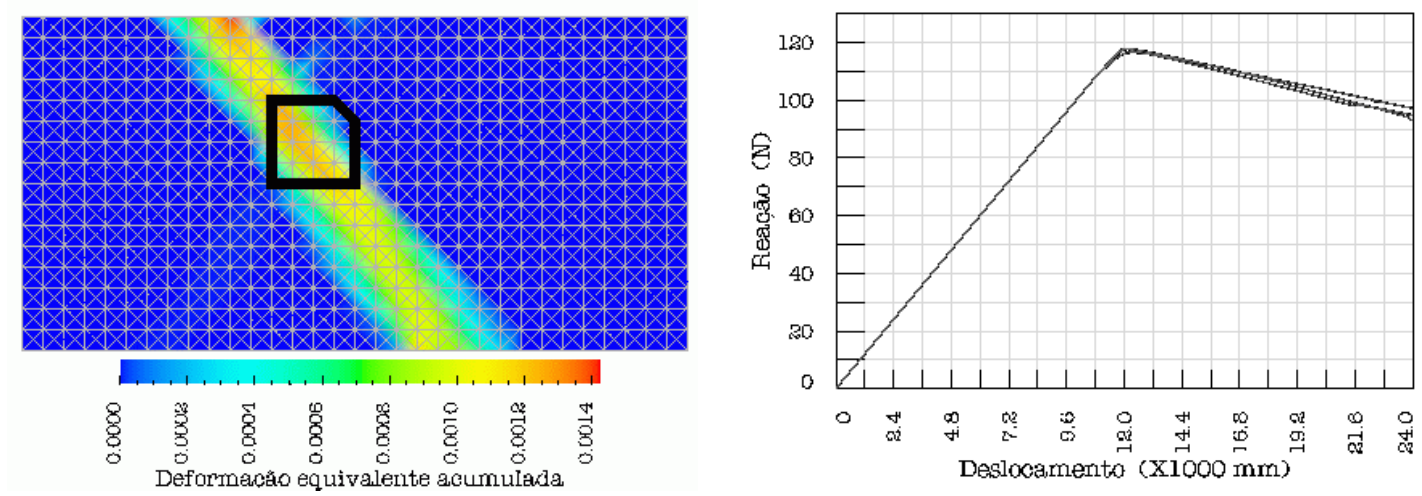

$\mathrm{h}=0.025 \mathrm{E}, \mathrm{w}=5000: 2048,512$ e 128 elementos

Figura 7.41 - Série 3: Plasticidade com gradiente: $w=5000$ N: $h=-0.025$ E.

A presença do gradiente regulariza os resultados para os diferentes tamanhos de malha. O tamanho, forma e posição do defeito é idêntico ao da figura anterior.

\subsubsection{Operador tangente contínuo}

Como complemento à apresentação dos resultados dos procedimentos implícitos, são apresentados exemplos utilizando-se o operador tangente contínuo, para a plasticidade com gradiente.

Os mesmos dados da segunda série e maior parte da série anterior são reutilizados nos exemplos seguintes. 


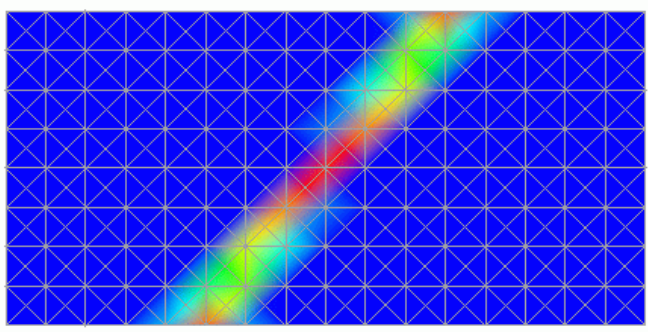

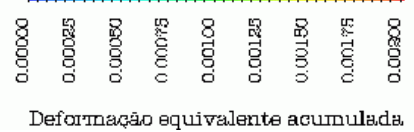

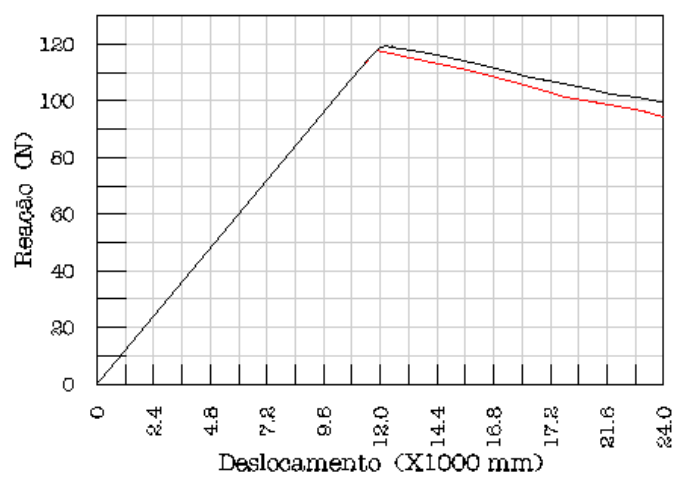

$\mathrm{W}=1,512$ elementos: operador algorítmico (em preto) $\mathrm{X}$ contínuo

Figura 7.42 - Série 3: w=1 N, 512 elementos, operador contínuo.

Resultados obtidos com o operador tangente consistente (em preto) são comparados aos do tangente contínuo. Pequena diferença para a simulação do caso local $(\mathrm{w}=1 \mathrm{~N})$.
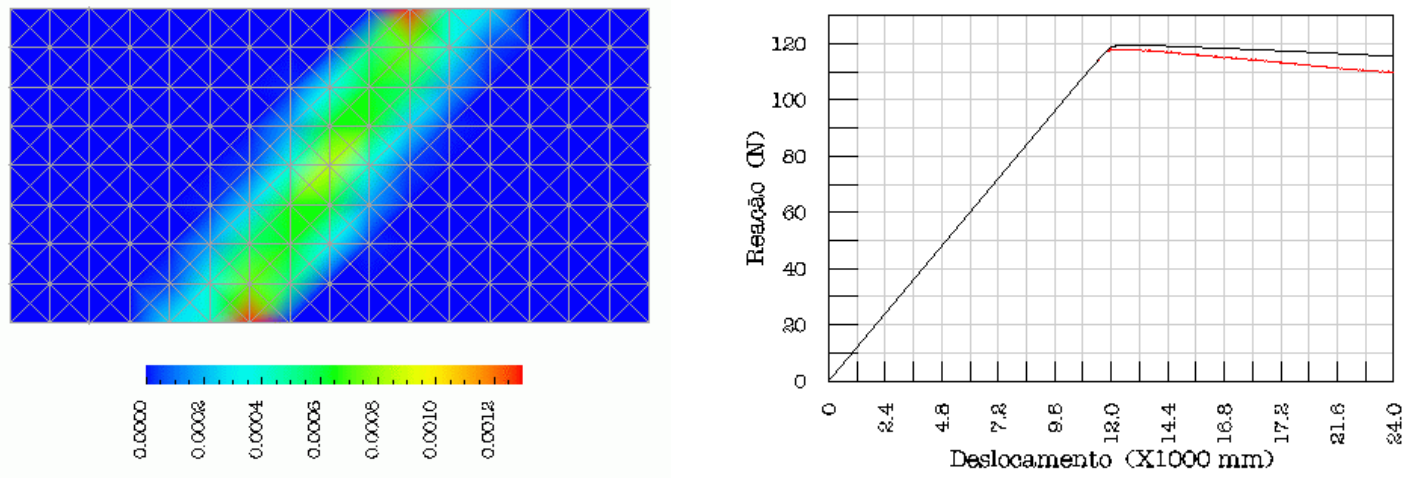

Deformacăo equivalente acumulada

$\mathrm{w}=10000,512$ elementos: operador algorítmico (em preto) $\mathrm{X}$ contínuo

Figura 7.43 - Série 3: w=10000 N; 512 elementos; operador contínuo.

As diferenças não são tão grande devido ao pequeno valor da constante de encruamento negativo. No entanto já é possível notar nas figuras um comportamento diferenciado nos resultados obtidos com os dois operadores. Em especial, a inclinação da parte descendente é diferente. 

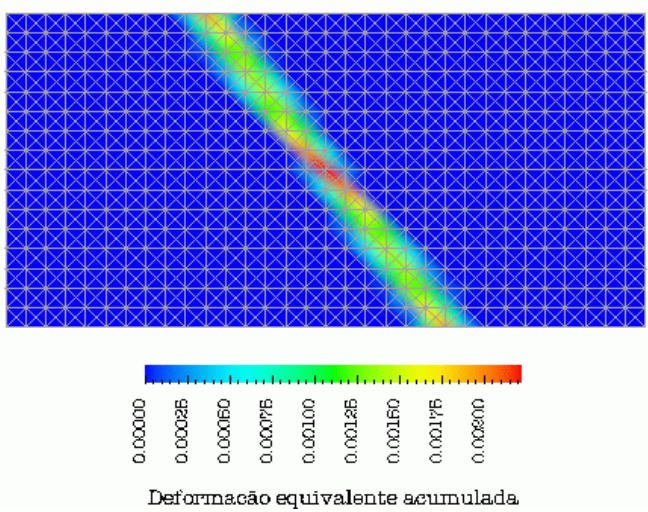

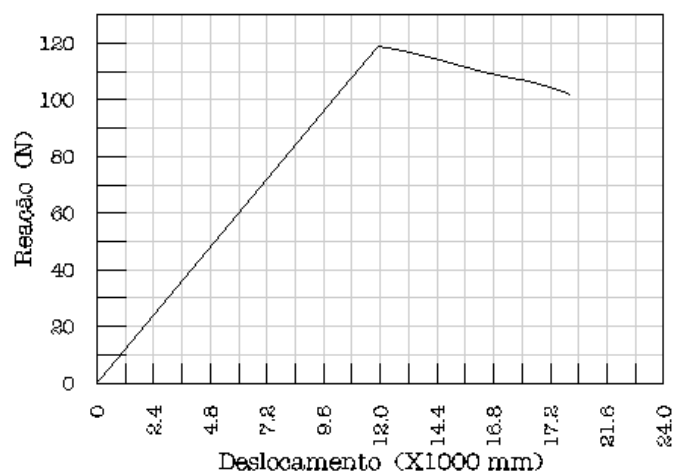

Figura 7.44 - Série 3: w=500 N; 2048 elementos; operador contínuo.

Neste caso apenas os resultados do operador tangente contínuo são mostrados no gráfico deslocamento $\mathrm{X}$ reação. A curva não termina no valor pretendido $(024 \mathrm{~mm})$ porque a solução numérica torna-se instável bem antes.

Embora os resultados obtidos com o operador em geral não fossem ruins, notou-se uma grande instabilidade do algorítmo. De fato, poucos exemplos numéricos puderam ser realizados, com valores mais críticos, em relação ao procedimento empregando o operador consistente.
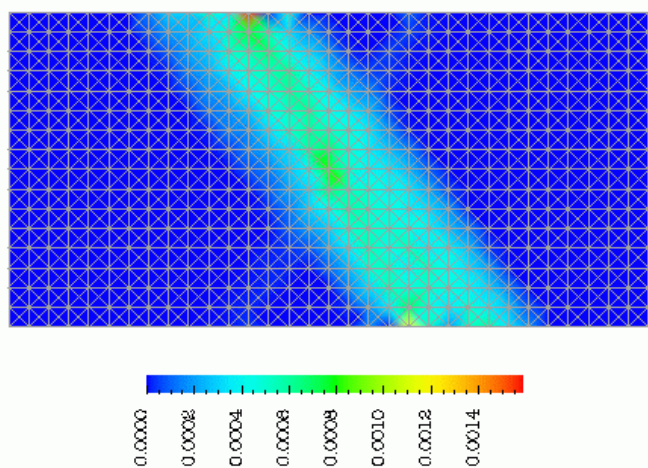

Deformacáo equivalente acumulada

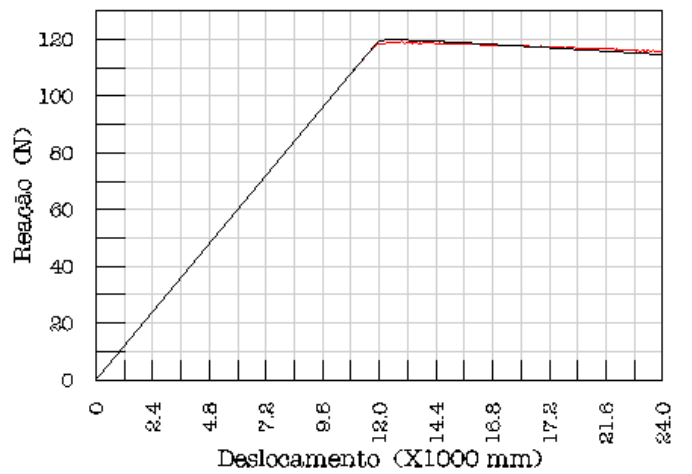

$\mathrm{W}=5000,512$ elementos: operador algorítmico (em preto) X contínuo

Figura 7.45 - Série 3: w=5000 N; 2048 elementos; operador contínuo.

Em exemplos em que o algorítmo com operador contínuo mostrou-se estável os resultados não foram ruins. Como é o caso deste exemplo, no qual o valor elevado 
de $\mathrm{w}$ favorece a coincidência dos resultado com o obtido com operador consistente. Além disso, valores elevados de w também favorecem a estabilidade do algorítmo com operador contínuo.

\subsection{Quarta série: localização induzida por condições de contorno}

O retângulo das séries anteriores continua considerado aqui, mas agora alterando-se as condições de contorno da Figura 7.1.

Nenhum ponto é enfraquecido, nos exemplos desta série. O retângulo é considerado como completamente homogêneo.

Apenas o coeficiente de Poisson é alterado, em relação às duas séries anteriores, para acentuar o efeito desejado.

O aparecimento da localização por efeito das condições de contorno contrasta com o efeito de imperfeições, simulado pelo enfraquecimento de algumas células, como no caso de todos os exemplos anteriores.

As novas condições de contorno são as da figura abaixo:

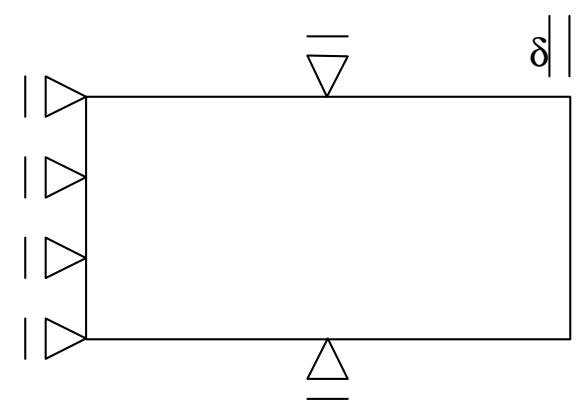

Figura 7.46 - Condições de contorno do retângulo homogêneo. 

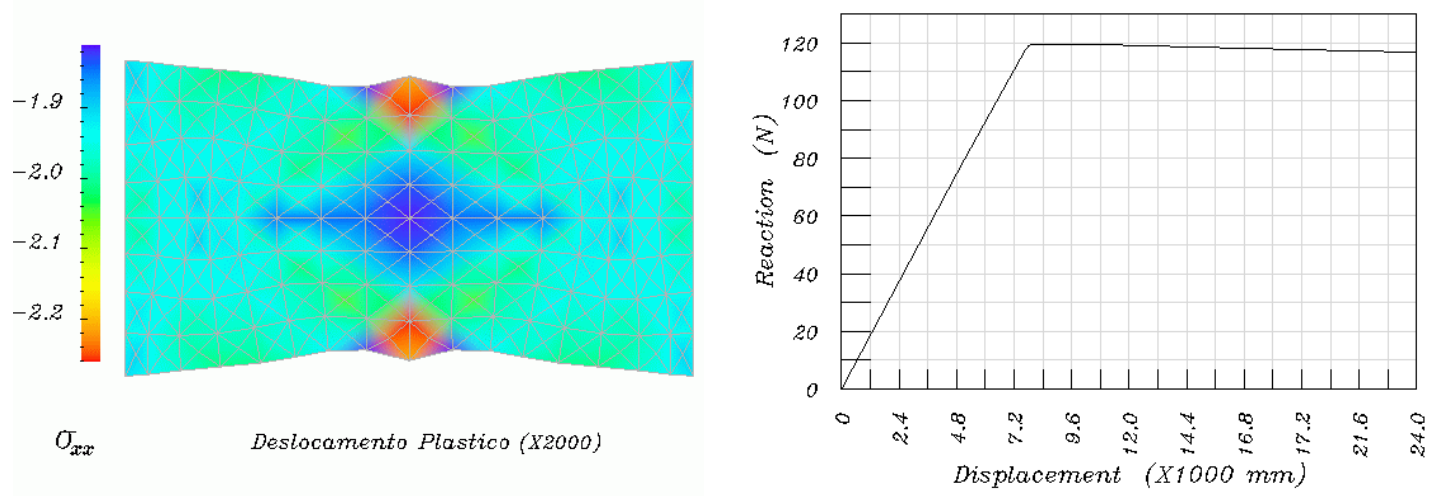

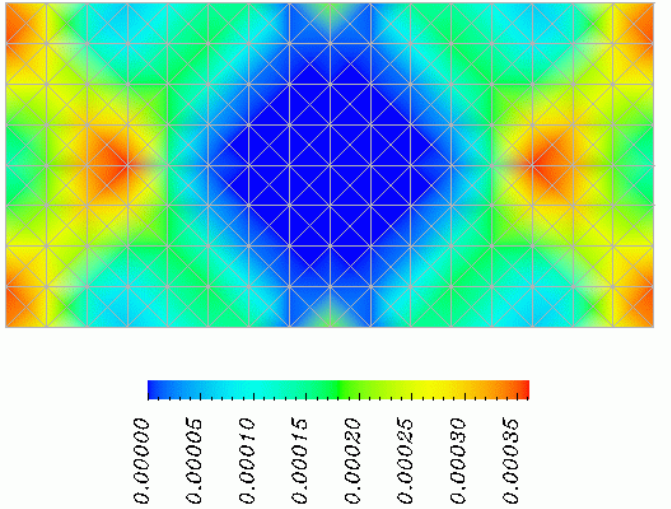

Deformacao equivalente acumulada

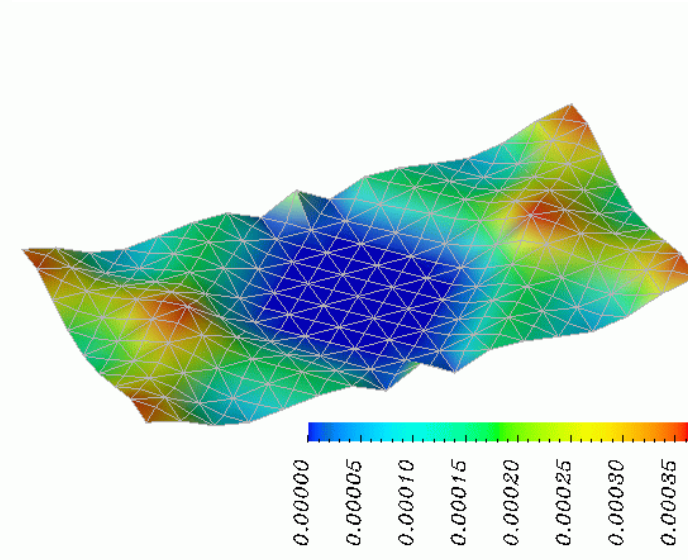

Deformacao equivalente acumulada

Figura 7.47 - Série 4: 512 células. Procedimento explícito. Plasticidade local. $\delta=0,024 \mathrm{~mm}, v=0,4999 \ldots 625$ incrementos de carga.

Apesar do passo de carga bastante pequeno, a localização é muito tênue neste caso.

Pode-se notar quatro faixas de localização. O passo de carga muito pequeno acaba induzindo preferência por uma das soluções, que neste caso ocorre por conta exclusivamente da simulação numérica, compreensível num processamento em tantos passos.

Observe-se o novo valor do coeficiente de Poisson, que é utilizado nesta última série de exemplos $(v=0,4999 \ldots)$. 

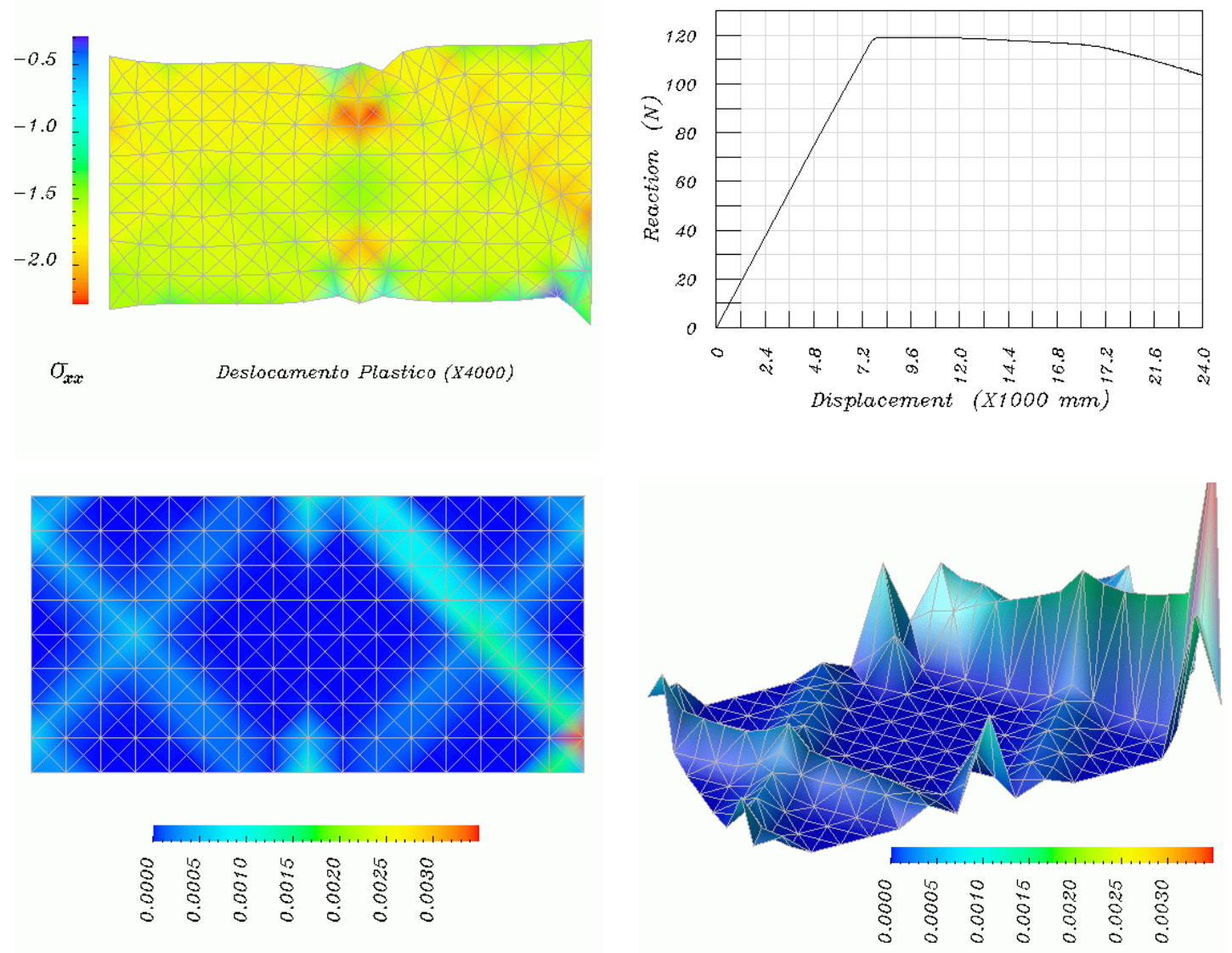

$\begin{array}{lllllll}8 & 6 & 0 & 1 & 0 & 1 & 0 \\ 8 & 8 & 8 & 8 & 0 & 0 & 8 \\ 8 & 8 & 8 & 8 & 8 & 8 & 8 \\ 0 & 0 & 0 & 0 & 0 & 0 & 0\end{array}$

Deformacao equivalente acumulada

Deformacao equivalente acumulada

Figura 7.48 - Série 4: 512 células. Procedimento implícito. Plasticidade local. $\delta=0,024 \mathrm{~mm}, v=0,4999 \ldots 625$ incrementos de carga.

Valem as mesmas observações da figura anterior. A diferença está no fato de que a localização de deformações neste caso é bem clara. 

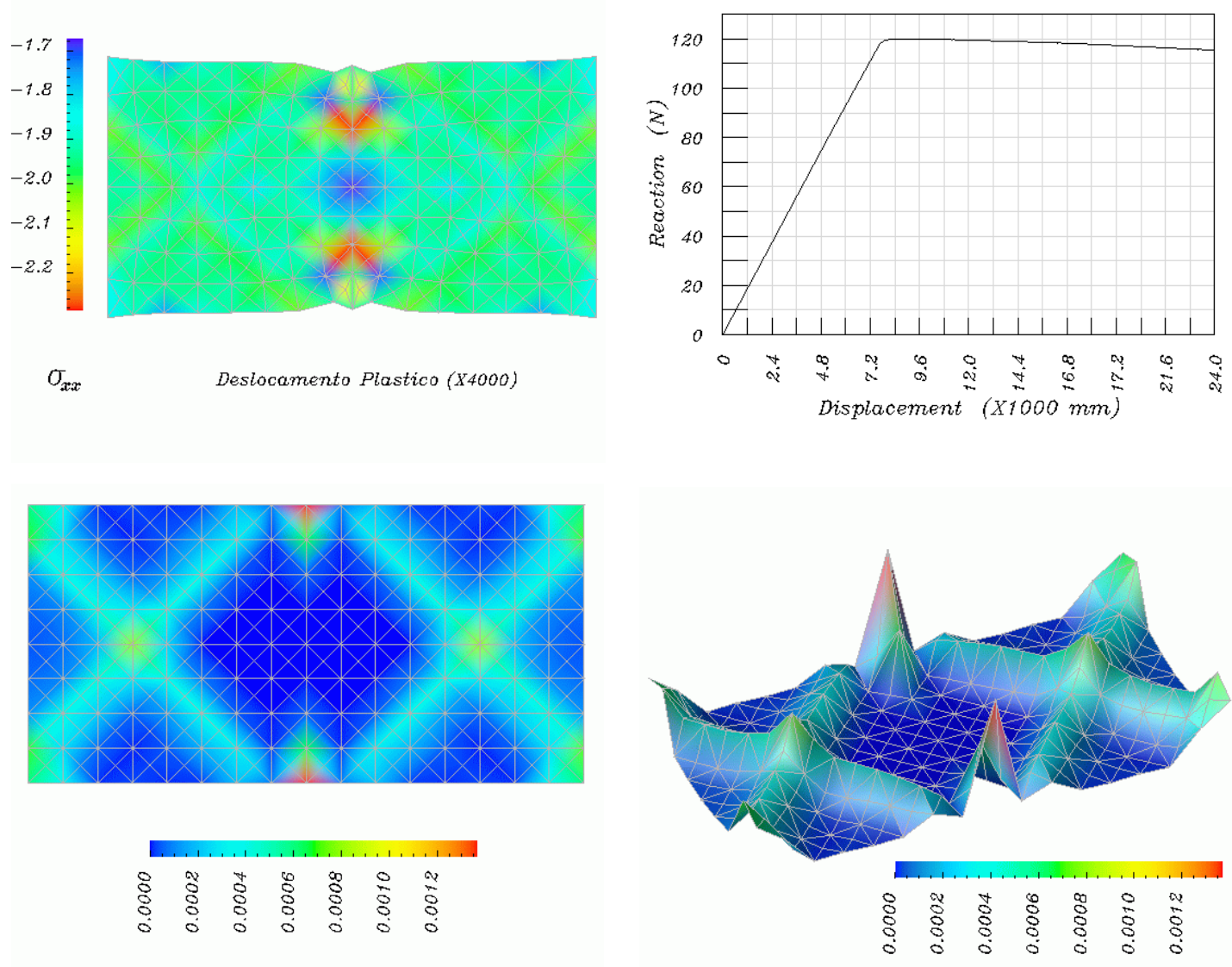

Deformacao equivalente acumulada

Deformacao equivalente acumulada

Figura 7.49 - Série 4: 512 células. Procedimento implícito. Plasticidade local. $\delta=0,024 \mathrm{~mm}, v=0,4999 \ldots 200$ incrementos de carga.

O menor número de passos de carga não induz uma faixa preferencial, e as quatro possibilidades apresentam-se como igualmente críticas. A presença dessas quatro soluções simultaneamente também dificulta a percepção da localização pela curva reação-deslocamento. 

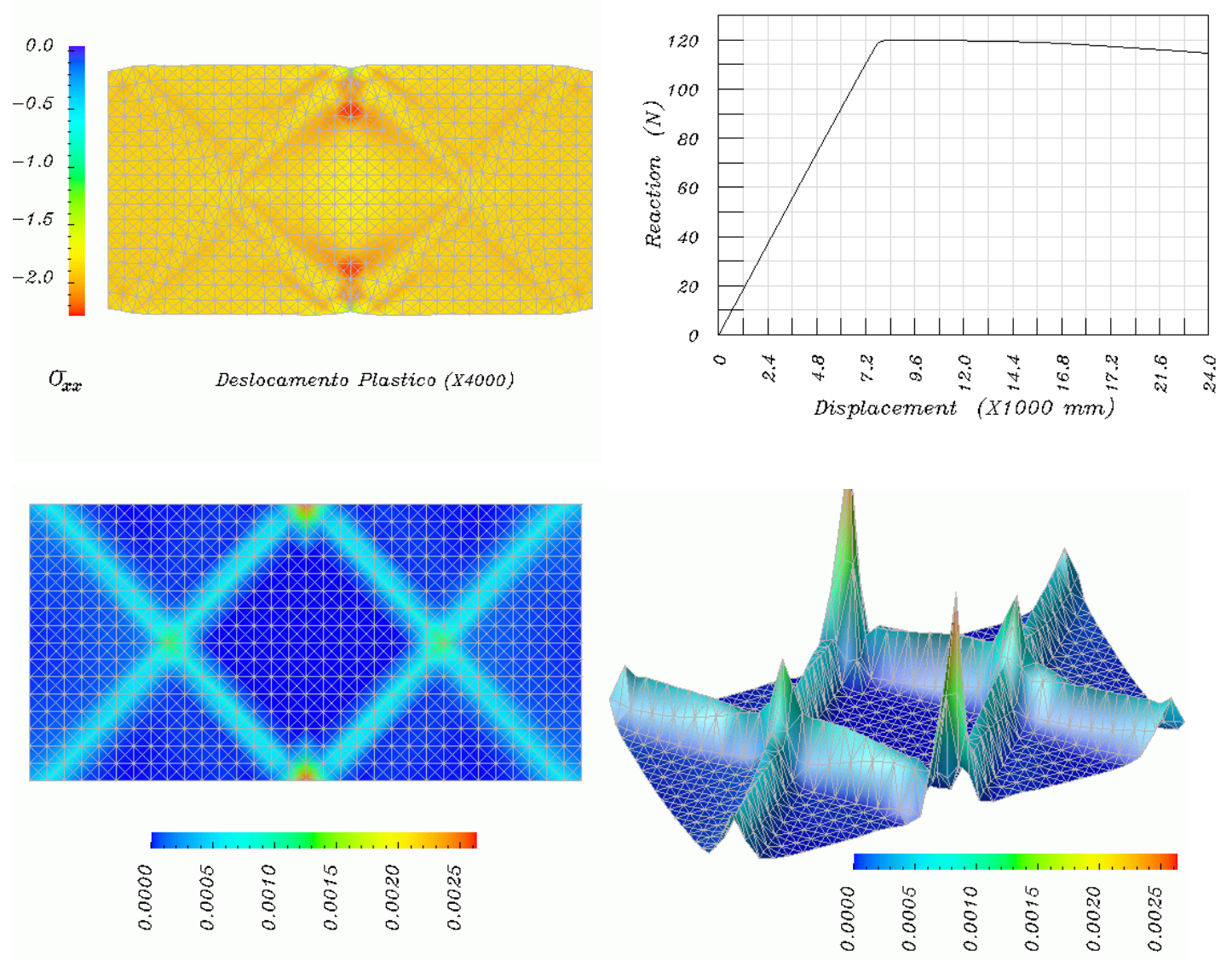

$$
\begin{array}{llllll}
8 & 6 & 0 & 6 & 0 & 0 \\
8 & 8 & 8 & 5 & 8 & 0 \\
0 & 0 & 8 & 8 & 8 & 8 \\
0 & 0 & 0 & 0 & 0 & 0
\end{array}
$$

Deformacao equivalente acumulada

Figura 7.50 - Série 4: 2048 células. Procedimento implícito. Plasticidade local. $\delta=0,024 \mathrm{~mm}, v=0,4999 \ldots 200$ incrementos de carga.

Os mesmos resultados da figura anterior, para a malha mais refinada. 


\section{Conclusões}

No início desta pesquisa, os objetivos principais eram o de estudar a regularização de soluções de problemas envolvendo a localização de deformações através do método dos elementos de contorno. Esta classe de problemas vem sendo bastante trabalhada nesta década no contexto do método dos elementos finitos. Portanto o objetivo central girava em torno da formulação desses problemas utilizando-se a formulação convencional dos elementos de contorno para problemas não lineares. Pretendia-se por exemplo, entre outras coisas, estender essa formulação também para modelos de dano.

No entanto, desde o início das implementações, constatou-se que a formulação tradicional apresentava resultados pouco satisfatórios. Mesmo no âmbito da plasticidade local, a formulação clássica apresentava dificuldades de precisão e de estabilidade numérica. Em problemas mais exigentes, com variações grandes e bruscas no campo de deslocamentos, surgiam dificuldades. Na implementação computacional inicial, o refinamento de malhas ainda que modesto por si só já trazia alguns problemas. Mas para lidar adequadamente com problemas como o da simulação da localização de deformações, porém, essas deficiências precisavam ser melhor resolvidas. A partir desse ponto, o enfoque do trabalho se deslocou, concentrando-se na obtenção de um programa operacionalmente funcional, confiável e robusto. As primeiras tentativas de melhorar o desempenho do programa computacional não apresentaram resultados satisfatórios, embora tenham constituído um passo na obtenção de um código mais robusto. Uma versão utilizando tensão constante nas células foi elaborada, sem que ocorresse nenhuma alteração significativa no padrão de comportamento do programa.

Neste contexto, uma das decisões foi a de implementar uma formulação utilizando-se a representação integral da tensão no contorno. Para problemas 
elásticos, isso é bastante importante, em certos casos, como o de concentrações de tensão, por exemplo. Não foi feita uma comparação com a forma de cálculo tradicional de tensões no contorno, envolvendo a derivada das funções de forma e valores obtidos para os deslocamentos nodais - e fica esta como uma primeira sugestão de continuidade dos trabalhos. No entanto, há alguns indícios de que isto possa ter tido um papel importante na melhora do comportamento do programa, conforme foi discutido no capítulo 4. Esta parece ter sido a primeira implementação da representação integral das tensões no contorno em problemas não lineares.

Uma outra linha de trabalho, na tentativa de melhorar os resultados das simulações, foi o de implementar um procedimento implícito para a solução do problema não linear. Inicialmente partiu-se para a formulação do procedimento implícito relativamente conhecida no contexto do método dos elementos de contorno. Essa formulação foi implementada, embora não tenha chegado a ser testada nem seu desempenho foi analisado. Esta constitui uma segunda sugestão de continuidade da pesquisa, ou seja, comparar as duas formulações do método implícito implementadas. Logo em seguida à primeira formulação e simultaneamente a sua implementação, começou-se a trabalhar na segunda formulação de um procedimento implícito do problema não linear, desta vez utilizando-se o conceito de operador tangente consistente. Teoricamente, o procedimento implícito com o operador tangente consistente é mais adequado, por garantir convergência quadrática ao processo iterativo. Neste trabalho o procedimento implícito utilizando operador tangente consistente foi estendido para o algorítmo de tensão inicial. Esta parece ter sido a segunda aplicação distinta do operador tangente consistente no procedimento implícito, no contexto do método dos elementos do contorno, ressaltando-se porém de que neste caso foi utilizada efetivamente uma formulação bidimensional, tanto na formulação quanto na apresentação de resultados numéricos.

Além disso, foi proposta e implementada uma formulação dos elementos de contorno para a plasticidade com gradiente utilizando o operador tangente consistente. Esta aplicação não é direta, devido ao fato de não de dispor se uma relação explícita para o multiplicador plástico, como na plasticidade local. Uma outra 
sugestão óbvia de continuidade da pesquisa é a implementação desta nova formulação.

No final do trabalho, o programa computacional apresentava um comportamento geral bem mais robusto, permitindo a simulação da localização mesmo utilizando-se o procedimento explícito da solução do problema não linear. Neste ponto foi possível finalmente retomar a proposta original do trabalho e testar a primeira implementação conhecida da plasticidade com gradiente através dos elementos de contorno. Os resultados a esse respeito não foram tão expressivos quanto o desejado, mas foram ao menos suficientes para mostrar como trabalha a introdução do segundo gradiente no comportamento da solução. Embora a localização tenha sido algo tímida utilizando-se o procedimento explicito, foi suficientemente clara, e o efeito do gradiente pôde ser constatado.

$\mathrm{Na}$ plasticidade com gradiente utilizando-se o operador tangente consistente os resultados obtidos foram excelentes. Do mesmo modo, na plasticidade local a superioridade dos resultados em relação ao procedimento explícito convencional é notável. A localização manifesta-se de maneira extremamente clara e acentuada, embora ainda haja vários aspectos a incluir na formulação - como a limitação do multiplicador plástico no modelo ou a consideração de "snap-back" (outras sugestões para a continuidade da pesquisa).

Enfim, o tempo diante do computador, trabalhando no código implementado e seus resultados, consumiu a maior parte da pesquisa. $\mathrm{O}$ resultado foi satisfatório, no sentido de que fenômenos como a localização de deformações podem ser simulados, e mesmo a plasticidade com gradiente apresenta seus primeiros resultados positivos.

Talvez a maior contribuição seja sintetizada na aplicação do operador tangente consistente num procedimento implícito, apresentando resultados muito expressivos com relação à formulação convencional. Se este tipo de resultados se repetir em outras aplicações do método, como deve ocorrer, então esta abordagem deverá se tornar um padrão, como já tem ocorrido há tempo no contexto dos elementos finitos.

Mas o método dos elementos de contorno cobra uma taxa extra em custo de implementação, como ilustra por exemplo a utilização da representação integral da 
tensão no contorno, discutida neste trabalho. Problemas formais deste tipo continuam em elaboração para o desenvolvimento do método. Essas dificuldades adicionais justificam em parte o relativo atraso em relação ao método dos elementos finitos como ilustra o caso da utilização do operador tangente consistente. No entanto é um custo que deve valer a pena, em vista das perspectivas oferecidas pelos elementos de contorno, ilustradas não apenas pela possibilidade de redução da discretização em muitos casos, como pela possibilidade de obtenção direta das tensões, em contraste com os métodos de deslocamentos. 


\section{Referências bibliográficas}

[1] ALIABADI, M. H. and ROOKE, D. P. (1991) Numerical Fracture Mechanics. Comp. Mech. Publications and Kluwer Academic Publischers.

[2] ARMERO, F. and GARIKIPATI, K. (1996) An analysis of strong discontinuities in multiplicative finite strain plasticity and their relation with the numerical simulation of strains localization in solids. Int. J. Solids Struct. 33, pp. 2863-2885.

[3] BAZANT, Z. P., BELYTSCHKO, T. B. and CHANG, T. P. (1984) Continuum theory for strain softening. J. Engng Mech. Div., ASCE 110, pp. 16661692.

[4] BAZANT, Z. P.; PIJAUDIER-CABOT, G. (1988) Nonlocal continuum damage, localisation instability and convergence. J. Appl. Mech., 55, pp 287-293.

[5] BENAllal, A., BILlARDON, R. and GEYMONAT, G. (1988) Some mathematical aspects of the damage softening problem. In: Cracking and Damage (ed. J. Mazars and Z. P. Bazant), pp. 247-258.

[6] BENALlAL, A., BILLARDON, R. and GEYMONAT, G. (1991) Localization phenomena at boundaries and interface of solids. In: Constitutive Laws for Engineering Materials (ed. C; S; Desai and G Frantziskonis), pp. 387-390. 
[7] BENALLAL, A. and TVERGAARD, V. (1995) Nonlocal continuum effects on bifurcation in the plane strain tension-compression test. J. Mech. Phys. Solids, vol 43, 5, pp. 741-770.

[8] BILLARDON, R. (1989) Étude de la rupture par la mécaniche de léndommagement. These de Doctorat, Université Paris 6.

[9] BONNET, M. and MUKHERJEE, S. (1996) Implicit BEM formulations for usual and sensivity problems in elasto-plasticity using the consistent tangent operator concept. Int. J. Solids Structures Vol 33, No. 30, pp 4461-4480.

[10] de BORST, R. (1988) Bifurcations in finite elemnt models with a nonassociated flow law. Int. J. numer. anal. Meth. geomech. 12, pp. 99-116.

[11] de BORST, R. (1991) Simulation of strain localisation: A reappraisal of the Cosserat continuum. Engng. Computations 8, pp 317-332.

[12] de BORST, R. and MUHLHAUS, H. B. (1992) Gradient-dependent plasticity: formulation and algorithmic aspects. Int. J. Num. Meth. Engng. 35, pp. 521-539.

[13] de BORST, R. (1993) A generalisation of $\mathbf{J}_{2}$-flow theory for polar continua. Comp. Meth. Appl. Mech. Ng., 103, pp 347-362.

[14] de BORST, R. PAMIN, J. and SLUYS, L. J. (1995) Gradient plasticity for localization problems in quasi-brittle and frictional materials. In: COMPLAS IV (ed. D. R. J. Owen, E. Onate, E Hinton) Pineridge-Press, Barcelona, 1, pp.535-546.

[15] BREBBIA, C. A., TELLES, J. C. F. and WROBEL, L. C. (1984) Boundary Element Techniques. Theory and Applications in Engineering. Springer-Verlag. 
[16] CHAVES, E. W. V., FERNANDES, G. R., VENTURINI, W. S. (1999) Plate bending boundary element formulation considering variable thickness. Engineering Analysis with Boundary Elements, to appear.

[17] COMI, C. and PEREGO, U. (1995a) A regularization technique for elastoplastic softening analyses based on generalized variables materials. In COMPLAS IV (ed. D. R. J. Owen, E. Onate, E Hinton) Pineridge-Press, Barcelona, 1,

[18] COMI, C. and PEREGO, U. (1995b) A generalized variable formulation for Gradient dependent softening plasticity. Int. J. Num. Meth. Engng, 39, pp. 3731-55.

[19] CRUSE, T. A.(1988) Boundary Element Analysis. In: Computational Fracture Mechanics, Kluwer Academic Publishers, Dordrecht.

[20] CRUSE, T. A.; SUWITO, W. (1993) On the Somigliana stress identity in elasticity. Computational Mech., 11, pp. 1-10.

[21] DRIEMEIER, L. (1999) Contribuição ao estudo da localização de deformações associada ao empego de modelos constitutivos de dano e plasticidade. Tese de Doutorado, EESC-USP.

[22] DRUCKER, D. C. (1959) A definition of stable inelastic material. J. Appl. Mech., 26, 101-106.

[23] ERINGEN, A. C. and EDELEN, D. G. (1972) On nonlocal elasticity. Int. J. Engng Sci. 10, pp. 233-248.

[24] FLECK, N. A. and HUTCHINSON, J. W. (1993) A phenomenological theory of strain gradient plasticity. J. Mech. Phys. Solids 41, pp. 1825-1857. 
[25] FUDOLI, C. A., BENALLAL, A, VENTURINI, W. S. (1996) Boundary Element method for plasticity. Laboratoire de Mécanique et Technologie (ENSCachan/CNRS/Paris 6), Rapport interne 175.

[26] FUDOLI, C. A., VENTURINI, W. S., BENALLAL, A. Boundary Element Formulation Applied to Localization Problems. In: Computational Mechanics: New Trends and Applications. Idelson,S., Oñate,E., Dvorkin,E. (eds.), CIMNE, Barcelona, (1998).

[27] FUDOLI, C. A., VENTURINI, W. S., BENALLAL, A. Implicit and explicit elastoplastic BEM formulations applied to localization. A ser publicado nos anais do COBEM 99 - IX Congresso Brasileiro de Engenharia Mecanica.

[28] FUDOLI, C. A., VENTURINI, W. S., BENALLAL, A. Implicit and explicit BEM formulations applied to gradient plasticity A ser publicado nos anais do $X X$ CILAMCE $-20^{\text {th }}$ Iberian Latin-American Congress on Computational Methods in Engineering.

[29] FUDOLI, C. A., VENTURINI, W. S., BENALLAL, A. Implicit and explicit BEM formulations for gradient plasticity and localisation phenomena. No prelo

[30] GUIGGIANI, M. and GIGANTE, A. (1990) A general algorithm, for multidimentional Cauchy principal value integrals in the boundary element method. J. Appl. Mech. ASME 57, pp. 906-915.

[31] GUIGGIANI, M., KRISHNASAMY, G., RIZZO, F. J. and RUDOLPH, T. J. (1990) Hypersingular boundary integral equations: a new approach to their numerical treatment. In: Boundary Integral Methods (ed. MORINO, L. and PIVA, R.), pp.211220, Berlin, Springer-Verlag. 
[32] GUIGGIANI, M., KRISHNASAMY, G., RIZZO, F. J. and RUDOLPH, T. J. (1992) A general algorithm for the numerical solution of hypersingular boundary integral equations. ASME J. Appl. Mech., 59, 604-14.

[33] GUIGGIANI, M. (1994) Hypersingular formulation for boundary stress evatulation. Engng. Analysis Boundary Element 13, pp. 169-179.

[34] HARTMANN, F. (1985) The Mathematical Foundation of Structural Mechanics. Springer-Verlag.

[35] HARTMANN, F. (1989) Introduction to Boundary Elements: Theory and Applications, Springer-Verlag, Berlin.

[36] HILL, R. (1958) A general theory of uniqueness and stability in elasticplastic solids. J. Mech. Phys. Solids 6, 236-264.

[37] LASRY, D. and BELYTSCHKO, T. (1988) Localization limiters in transient problems. Int. J. Solids Struct. 24, pp. 581-597.

[38] LUTZ, E., GRAY, L. J. and INGRAFFEA, A.R. (1990) Indirect evaluation of surface stress in the boundary element method. In: Boundary Integral Methods (ed. MORINO, L. and PIVA, R.), pp. 211-220, Berlin, Springer-Verlag.

[39] MAIER, G., MICCOLI, S.. NOVATI, G. and PEREGO, U. (1995) Symmetric Galerkin boundary element method in plasticity and gradient plasticity. Comp. Mech. 17, pp. 115-129.

[40] MALVERN, L. E. (1969) Introduction to the Mechanics of a Continuous Medium. Prentice Hall.

[41] MUHLHAUS, H. B. and AINFANTIS, E. (1991) A variational principle for gradient plasticity. Int. J. Solids Struct. 28, pp. 845-857. 
[42] MANZOLI, O. L. (1998) Un modelo analítico y numérico para la simulación de discontinuidades fuertes en la mecánica de sólidos. Tesis Doctoral, Universitat Politècnica de Catalunya.

[43] ORTIZ, M.; LEROY, Y. and NEEDLEMAN, A. (1987) A finite element method for localized failure analysis. Int. J. Num. Meth. Eng., 23, 353-366.

[44] PAMIN, J. P. (1994) Gradient dependent plasticity in numerical simulation of localization phenomena, PhD Thesis, Delft University Press.

[45] PIJAUDIER-CABOT, G. and BENALLAL, A. (1993) Strain localization and bifurcation in a nonlocal continuum. Int. J. Solids Structures, Vol 30, 13, pp. 17611771.

[46] POON, H., MUKHERJEE, S., BONNET, M. (1998) Numerical implementation of a CTO-based implicit approach for the BEM solution of usual and sensitivity problems in elasto-plasticity. Engineering Analysis with Boundary Elements 22, 257-269.

[47] PROENÇA, S. P. B. Análise não linear de estruturas, Notas de aula. EESCUSP.

[48] RICE, J. R. (1976) The localization of plastic deformation. In: Theoretical and Applied Mechanics (ed. W. T. Koiter), pp. 207-220, North Holland Publ. Co.

[49] RICHARDSON, J. D. (1998) Weakly singular BEM for fracture modeling. PhD Thesis, Vanderbilt University.

[50] RUDNICKI, J. W. and RICE, J R. (1975) Conditions for the localization of deformation in pressure sensitive dilatant materials. J. Mech. Phys. Solids, 23, pp. 371-394. 
[51] SIMO, J. C.; TAYLOR, R. L. (1985) Consistent tangent operators for rateindependent elastoplasticity. Comp. Meth. Cech. Engng. 48, pp 101-118.

[52] SHEWCHUK, J. R. (1996) Triangle: engineering a 2D quality mesh generator and Delaunay triangulator. First workshop on applied computational geometry. (Philadelphia, Pennsylvania), ACM. pp. 124-133.

[53] SHEWCHUK, J. R. (1996) http://www.cs.cmu.edu/ quake/triangle.html.

[54] TELLES, J. C. F. (1983) The Boundary Element Method Applied to Inelastic Problems. (Lecture Notes in Engineering) Springer-Verlag.

[55] TELLES. J. C. F.; CARRER, J. A. M. (1991) Implicit procedures for the solution of elastoplastic problems by the boundary element method. Math. Comput. Modelling 15, pp 303-311.

[56] TELLES. J. C. F.; CARRER, J. A. M. (1994) Static and transient dynamic nonlinear analysis by the boundary element method with implicit techniques. Engng. Anal. Bound. Elem. 14, pp 65-74.

[57] TRIANTAFYLLIDIS, N. and AINFANTIS, E. (1986) A gradient apprach to localization of deformation. I-Hyperelastic materials. J. Elasticity 16, pp. 225-237.

[58] VENTURINI, W. S. (1983) Boundary Element Method in Geomechanics. (Lecture Notes in Engineering) Springer-Verlag.

[59] ZHU, J. and SHAH, A. H. (1996) The evaluations of Cauchy principal value integrals and weakly singular integrals in BEM and their applications. Int. J. Num. Meth. Engng. 39, pp. 1017-1028. 